\title{
The Influence of Spatial Attention on Neuronal Receptive Field Structure within Macaque Area MT
}

\author{
Dissertation \\ zur Erlangung des Doktorgrades \\ der mathematisch-naturwissenschaftlichen Fakultäten \\ der Georg-August Universität Göttingen
}

vorgelegt von

DiPL. PSYCH.

Thilo WOMELSDORF

aus Bad Oeynhausen

Göttingen 2004 
D 7

Referent:

Korreferent:
Prof. Dr. S. Treue

Prof. Dr. U. Jürgens

Tag der mündlichen Prüfung: $\quad$ Thursday, 04.11.2004 


\title{
Contents
}

\author{
Contents
}

List of Figures

viii

List of Tables

1 Introduction

1.1 Receptive Field Properties of Area MT . . . . . . . . . . . . . 6

1.1.1 Macaque Area MT: Anatomical and Functional Properties . . 6

1.1.2 Spatial RF Properties of Area MT . . . . . . . . . . 8

1.2 Attentional Modulation within Visual Cortex . . . . . . . . . . . 13

1.2.1 Psychophysics of Spatial Attention . . . . . . . . . . 13

1.2.2 A Brief History on Top-Down Modulation . . . . . . . . . . . 15

1.2.3 Attentional Gain Modulation \& Sensitivity Enhancement . . . 15

1.2.4 Push-Pull Effect of Selective Attention: Gain versus Competition . . . . . . . . . . . . . . . 17

1.2.5 Sites of Attentional Modulation: Input Modulation, Hierarchical Gradient \& Adjustable Spotlight . . . . . . . . . . . . 22

1.2.6 Cortical Representation of Spatial Saliency . . . . . . . . . . 26

1.2.7 Beyond Space as the Unit of Selection: Feature- and ObjectBased Attention . . . . . . . . . . . . . . . . 29

1.2 .8 Summary . . . . . . . . . . . . . . . . . . 31

1.3 Dynamic Plasticity of Spatial RF Tuning . . . . . . . . . . . . . . 32

1.3.1 Selective Attention and Spatial Tuning . . . . . . . . . . 32

1.3.2 Intention and Attention: RFs during Directional Movements . 34

1.3.3 Effects of Expectation and Alertness . . . . . . . . . . . 37

1.3.4 Dynamics at Longer Time Scales: Conditioning, Adaptation and Context-Dependent Plasticity . . . . . . . . . . . 39

1.4 Summary \& Hypothesis . . . . . . . . . . . . . . . . . 43

2 Materials \& Methods $\quad 45$

2.1 Experimental Settings . . . . . . . . . . . . . . . 45 
2.1.1 Electrophysiological Specification . . . . . . . . . . 45

2.1.2 Confirmation of Recording Sites . . . . . . . . . . . . 46

2.1.3 Stimulus Presentation \& Behavioral Control . . . . . . . . . 50

2.2 Experimental Protocol and Conditions . . . . . . . . . . . . . 50

2.2.1 Attentional RF Mapping . . . . . . . . . . . . . . 51

2.2.2 Direction Tuning: Motion Reverse Correlation . . . . . . . . . 53

2.3 Data Analysis . . . . . . . . . . . . . . . . . . . . . 54

3 Results $\quad 59$

3.1 Sensory Properties . . . . . . . . . . . . . . . . . . . 59

3.1 .1 Directional Tuning . . . . . . . . . . . . . . 59 59

3.1.2 Spatial Tuning With and Without Multiple Stimuli . . . . . . 62

3.2 Spatial Attention Effects . . . . . . . . . . . . 68

3.2.1 Behavioral Performance . . . . . . . . . . . . . . 68

3.2.2 Modulation of Response Strength . . . . . . . . . . . . . 68

3.3 Attentional Effects on Spatial Sensitivity . . . . . . . . . . . . 71

3.3.1 Inside-Inside Comparison: RF Shift . . . . . . . . . . . . . . 72

3.3.2 Inside-Outside Comparison: Directionality and Magnitude of RF Shift . . . . . . . . . . . . . . . . . 76

3.3.3 Spatial Specificity: Shift Asymmetry . . . . . . . . . . . 82

3.3.4 RF Size Changes and their Relation to RF Shifts . . . . . . . 84

3.3.5 RF Size Changes and Modulation of Gain . . . . . . . . . . 87

3.4 Correlation of RF Changes with Performance . . . . . . . . . . . 90

3.5 Time Course of Spatial RF Shift . . . . . . . . . . . . . . . 93

3.6 Eye Position Analysis . . . . . . . . . . . . . . . . . . . 96

$\begin{array}{llr}4 & \text { Discussion } & 99\end{array}$

4.1 Directional Tuning: Spike-Triggered Averaging and Classical Methods 99

4.2 Sensory RF Maps . . . . . . . . . . . . . . . . . . . . . . . . . 100

4.3 Attentional Influence on Spatial Tuning: Summary of Results . . . . 102

4.4 Neuronal Implications of Spatial Tuning Shifts . . . . . . . . . . . . 107

4.4.1 Attentional Input Modulation and Spatial Routing of Information . . . . . . . . . . . . . . . 107

4.4.2 Attentional RF Recruitment, Cortical Magnification, and Spatial Resolution . . . . . . . . . . . . . . . . . . . 111

4.5 Perceptual Implications of Attentional RF Recruitment . . . . . . . . 114

4.5.1 Spatial Distributions of Attention . . . . . . . . . . . . . . . 114

4.5.2 Spatial Distortions with Focal Attention . . . . . . . . . . 117

4.5.3 Spatial Resolution of Attention . . . . . . . . . . . . 118

4.6 General Summary and Conclusion . . . . . . . . . . . . . . . . 119 
Appendix

$\begin{array}{ll}\text { A Difference Map Examples } & 123\end{array}$

\begin{tabular}{l|l} 
B Recorded Cells: Overview of Experiments & 127
\end{tabular}

\begin{tabular}{l|l} 
Acknowledgements & 143
\end{tabular}

\begin{tabular}{l|l} 
Bibliography & 176 \\
\hline
\end{tabular}

$\begin{array}{lr}\text { Curriculum Vitae } & 177\end{array}$ 


\section{List of Figures}

2.1 Histology: Summary . . . . . . . . . . . . . . . . . 48

2.2 Histology: Confirmation of Recording Site . . . . . . . . . . . . . . 49

2.3 Experimental Design . . . . . . . . . . . . . . . . . . . . . . 52

2.4 Motion Reverse Correlation Method . . . . . . . . . . . . . . . 55

2.5 One-Dimensional RF Projection: Illustration . . . . . . . . . . . . . 57

3.1 Characterisation of Direction-Tuning . . . . . . . . . . . . 60

3.2 Spike-Triggered Direction-Tuning: Examples . . . . . . . . . . . . . 61

3.3 Sensory RFs: Effect of Null Directions of Motion . . . . . . . . . . . 64

3.4 Sensory RFs: Difficulty and Feature-Based Effects . . . . . . . . . . . 66

3.5 Behavioral Performance . . . . . . . . . . . . . . . . 69

3.6 Attentional Modulation of Response Strength . . . . . . . . . . . . . 70

3.7 Average PSTH to Probe Stimuli . . . . . . . . . . . . . . . . . 71

3.8 Illustration of Spatial Shift: Inside-Inside . . . . . . . . . . . . . 73

3.9 Shift of Centroid and Peak (Angle): Inside-Inside . . . . . . . . . . 74

3.10 Shift of Centroid and Peak (Distance): Inside-Inside . . . . . . . . . . 76

3.11 Shift of Centroid and Peak: Inside-Outside . . . . . . . . . . . . 78

3.12 Shift of RF-Peak Response: Overview . . . . . . . . . . . . . . . . . . 79

3.13 Vector Distributions of RF-Peak Responses: Inside vs. Outside . . . . 81

3.14 Vector Distributions of RF-Peak Responses: Inside vs. Fovea . . . . . 82

3.15 Shift Magnitude and RF Separation from Attended Stimulus . . . . . 84

3.16 RF Size Modulation with Attention . . . . . . . . . . . . 85

3.17 RF Size Changes and RF Shifts . . . . . . . . . . . . . . . 86

3.18 RF Size Changes and its Relation to Gain Changes . . . . . . . . . . 88

3.19 RF Size Changes and Performance Differences . . . . . . . . . . . . . 92

3.20 Spatial Reverse Correlation Analysis . . . . . . . . . . . . . . . . 94

3.21 Examples of Eye Position Distribution for Attention Conditions . . . 96

3.22 Eye Position Deviation: Attend Inside Conditions . . . . . . . . . . . 98

4.1 Spatial Retinotopy of Attention in V1 . . . . . . . . . . . . . . . 108

4.2 Neuronal Propagation of Top-Down Modulated Activity . . . . . . . 109 
4.3 Illustration of Attentional Magnification of Peripheral Space . . . . . 113

4.4 Gradient of Spatial Attention: Psychophysics . . . . . . . . . . . . . . 115

A.1 Illustration of Difference Map Computation . . . . . . . . . . . . . . 124

A.2 Examples of Difference Maps . . . . . . . . . . . . . . . . . . . . . . 125 


\section{List of Tables}

3.1 RF Shift Results: Inside-Inside . . . . . . . . . . . . . 75

3.2 RF Shift Results: Inside-Outside . . . . . . . . . . . . 79

3.3 RF Shift Results: Proportions of Significant Effects . . . . . . . . 83

B.1 Recorded Cells: Overview . . . . . . . . . . . . . . . . . . 130 


\section{Chapter 1}

\section{Introduction}

Receptive fields (RFs) constitute the central mechanisms of the nervous system to transform incoming information into representations that can be used to control adaptive behavior. Within the visual system the transformation is based on RFs of neurons with two general properties: They are responsive to a limited spatial region, and are selectively tuned to a subset of visual stimuli with particular featural information (e.g. motion direction, color, orientation). Neuronal RFs thus integrate visual information from specific regions in space and encode featural content at that location by means of response strength. This architecture would in principle allow to decode the spatial location and feature of a visual stimulus by finding the neuronal $\mathrm{RF}$ with the best overlapping properties. However, within natural environments local regions in space typically contain more than one stimulus and each stimulus is constituted of various features. Sensory responses of neuronal RFs overlapping spatial regions that encompass multiple stimuli are typically ambiguous with regard to the identity of the individual stimuli: Similar response magnitudes are obtained with dissimilar sets of visual stimuli within the RF. How are these neurons then capable to contribute to the selective representation of relevant visual information?

Neurophysiological work from the past two decades has revealed two major mechanisms to resolve this conflict at the single neuron level: (i) A stimulus-driven, or bottom-up mechanism that enhances responses to local feature contrasts based on antagonistic center-surround organization of neuronal RFs, and (ii) top-down attentional influences that selectively modulate sensory responses of RFs overlapping with behaviorally relevant stimuli. This thesis is concerned with the second, top-down influence on sensory processing. In particular, selective attention has been shown to reduce the ambiguity in coding multiple stimuli within one RF dramatically. When attending to one of two or more stimuli within a RF of a neuron, an otherwise ambiguous response becomes more like the response that would be obtained when only the attended stimulus is within the RF of a neuron. While this influence of selective attention on neuronal responses highlights its central role in visual processing, the 
underlying mechanism are not well understood. One theoretical account emphasizes a spatial mechanism underlying the changes in response magnitude with attention. Proponents of this model suggest that attention involves RFs of sensory neurons to conjointly shift toward and shrink around the attended target (Moran and Desimone, 1985; Reynolds and Desimone, 1999). RF shrinkage around attended RF stimuli would reduce the influence of unattended stimuli and thus make responses similar to the response obtained when only the attended stimulus is presented to the RF. Alternatively, attention might not shrink RFs, but may solely shift, or scale RFs in magnitude by means of a different and more parsimonious mechanism: The introduction of a general gain on responses of neurons tuned to the attended feature and/or location is likewise able to account for the observed magnitude modulation of attention (Treue and Martinez-Trujillo, 1999; Maunsell and McAdams, 2001). The project of this thesis was set out to clarify the underlying mechanisms of selective attention. In particular, it aims at establishing empirical evidence of the role of spatial mechanisms underlying attentional modulation. The major question will be whether, and how, spatial attention changes the spatial layout of RFs in extrastriate visual cortex when one of two closely adjacent visual stimuli within the RF is attended.

Data on these putative spatial mechanisms of visual attention have implications for various general aspects of selective visual information processing. These include (i) the relevance of inhibitory neuronal interactions, (ii) the spatial distribution and resolution of attentional effects, (iii) the propagation of selective representations along hierarchical processing chains, and (iv) the binding of distributed information to unified percepts. The next paragraphs introduces these diverse aspects as a precursor of the following sections. Later sections will survey the background of these general aspects in more detail before the hypothesis and design of the current project are introduced.

\section{Neuronal mechanisms of selective attention: the role of inhibition}

As already insinuated at in the previous paragraph two main diverging views on the neuronal mechanism of attention have been put forward, which might be summarized as the biased competition hypothesis and the gain hypothesis of selective attention. Both approaches share many empirical predictions on attentional influences on sensory responses. However, an influence of attention on the size and spatial position of RFs, i.e. a RF shrinkage, is suggested primarily by the biased-competition hypothesis, which has even been build around the assumption of RF shrinkage (Desimone and Duncan, 1995; Luck et al., 1997b; Reynolds and Desimone, 1999). Gain models, on the other hand, do not require a special spatial mechanism to account for attentional modulation (Hillyard, Vogel, and Luck, 1998; Treue and Martinez-Trujillo, 1999; Maunsell and McAdams, 2001; Salinas and Thier, 2000; Freeman et al., 2002). 
The biased competition hypothesis supposes that RF shrinkage implicates attentional signals in extrastriate visual area to actively inhibit neurons in the afferent populations. However, despite this core assumption of a spatial mechanism underlying that hypothesis there is currently no direct empirical evidence that the observed modulation of response magnitude is due to a spatial shrinkage of RFs. While the current project is not set up to decide between the different models, it attempts to provide the missing empirical basis for theoretical specifications about the role of spatial attentional mechanisms.

\section{Spatial distribution and resolution of attention}

Attentional selection of a particular region in space typically enhances sensitivity, accuracy and response speed for stimuli at attended locations (e.g. Carrasco, Ling, and Read 2004). Moreover, perceptual facilitation is often not restricted to stimuli at the focus of attention but is also evident in its vicinity. The distribution of attentional influences has therefore been equated with a spotlight, a zoom lens, or as a spatial gradient centered at the attentional focus (Posner, Snyder, and Davidson, 1980; Eriksen and St.James, 1986; LaBerge et al., 1997; Cave and Bichot, 1999). In addition to this facilitatory gradient, various psychophysical findings indicate that perceptual performance is particularly vulnerable to interference of distracting stimuli in the surround of the focus of attention (e.g. Cutzu and Tsotsos 2003). Both, facilitatory and inhibitory aspects of the spatial distribution of attention have also been illustrated with functional MRI in human subjects (Tootell et al., 1998; Brefcynski and DeYoe, 1999; Müller et al., 2003; Slotnick et al., 2002; Slotnick, Schwarzbach, and Yantis, 2003). However, the neural mechanisms of the distribution of spatial attentional effects remain poorly understood with methods of low spatial resolution of brain activity (like fMRI). Neural correlates of the spotlight of attention might rather be evident at the level of single neuronal RFs that changes their spatial sensitivity according to the position and grain of the attentional focus. Thus, extracellular recording of the detailed RF outline during the deployment of selective attention will be capable of shedding light on likely neuronal mechanisms underlying attentional processes.

\section{Propagation of attentionally modulated responses along processing hierarchies} Studying attentional influences on neuronal RFs impinges also on additional aspects of visual information processing. Selective attention exerts progressively stronger influence on neuronal responses at successively higher sensory areas along the visual processing hierarchy (Maunsell and Cook, 2002). This finding has been suggested to reflect that attention acts strongest on neurons whose properties are particularly matched to the stimulus and requirements of the attentional task (Treue and Maunsell, 1996; Cook and Maunsell, 2002b). According to this view, the cognitive operations involved in attentional tasks will induce attentional influences in neurons 
with properties matching the complexity required by the task. However, in addition to task requirements a major mechanisms underlying the attentional gradient might be a spatial weighting based on dynamic changes of RFs: If at each processing level neuronal RFs would shift toward the attended location, the convergence of efferent output to successive stages would additionally increase the selectivity of the representation: Neurons with RFs devoted to encode unattended locations will progressively have less influence on responses at later stages. The net result would be an attentional gradient which parallels the hierarchical gradient along the processing pathways. The project of the current thesis investigates the nature of this spatial weighting and might thus contribute to the general question why representations at later stages of processing become increasingly selective (i.e. response modulation is stronger).

\section{Resolving perceptual ambiguities and the binding problem}

The representation of relevant spatial regions within the visual field is at the core of a variety of psychophysical and computational models of selective attention (Olshausen, Anderson, and van Essen, 1993; Niebur and Koch, 1998; Itti and Koch, 2001). The feature integration theory of attention by Treisman is one of the most widely cited approaches in this field (Treisman and Gelade, 1980; Treisman, 1998). This model assumes that visual features are encoded in independent and specialized feature maps, consistent with neurophysiology. This architecture calls upon a general mechanism to combine information from different features in order to establish the representation of a whole object which is constituted of different features, i.e. a particular color, form, and motion direction. Treisman suggested that spatial attention provides the core mechanism: The representation of the attended location in an explicit spatial saliency map provides the basis to combine featural information into unified objects. Thus, spatial attention could solve what has become known as the more general binding problem. Indeed, Reynolds and Desimone (1999) suggested that shrinking RFs could solve (, or at least ameliorate) the binding problem: When cortical RFs could adjust their resolution to the subspace containing the relevant object they would signal exclusively information about that object and could thus serve as a link for other distributed featural information pertaining to that object. According to this suggestion the experiment of the current project will have some bearing on very general aspects of the neuronal mechanisms underlying perceptual organization.

The previous paragraphs highlight the potential relevance of attentional effects at the single neuron level for an understanding of selective visual information processing. The following introductory sections of this thesis will survey in more detail the current knowledge about the visual area that is the target of this research (section 1.1) and the contribution of neurophysiological studies on selective attentional 
influences on visual information processing (section 1.2). A separate section will then discuss the putative influence of dynamic aspects of cortical RFs for selective representation of visual information (section 1.3). Before commencing with selective aspects of visual processing, the following section provides background information on the properties of neuronal RFs within area MT. 


\subsection{Receptive Field Properties of Area MT}

Visual information processing is based on cortical RFs within a hierarchy of functionally specialized visual areas of increasing complexity as one proceeds along the hierarchy. Two broadly defined pathways have been identified within the visual system, which are segregated with regard to functional specialisation and based on anatomic connectivity (Fellemann and van Essen, 1991). The occipito-temporal pathway is constituted of RFs with properties reflecting increasingly complex featural attributes that make up visual objects including form, texture and color. The second, occipito-parietal pathway, on the other hand, is constituted of neuronal RFs which are devoted to encode spatial locations, motion, and binocluar disparity among a general functional involvement into 'how' this information is used for behavior in contrast to 'what' the identity of visual information is (Goodale and Milner, 1992; Maunsell, 1992).

The anatomical target area of the current project is located at an intermediate stage of the occipito-parietal pathway. More specifically, the mid temporal area (area MT) is in the upper, lateral bank of the superior temporal sulcus of the macaque brain. Area MT is an anatomically and functionally well characterized extrastriate visual area with various properties that are especially relevant for the current project: Neuronal activity in area MT is (i) highly feature selective, (ii) has been shown to be functionally involved in motion processing, and (iii) there is specific knowledge of the spatial summation properties of single neurons. This section will briefly survey these aspects in order to provide a background before the following section will introduce the known influence of attention on sensory responses of area MT neurons.

\subsubsection{Macaque Area MT: Anatomical and Functional Prop- erties}

Area MT is located at an intermediate position within the dorsal parietal pathway. The main cortical antecedent areas projecting to area MT may be grouped into three classes: afferents arising directly from V1 (predominantly from layer IV cells), connections from (mainly) the thick stripes of area V2, and a large proportion of direct inputs from area V3 and V3A (Tootell et al., 1983; Maunsell and van Essen, 1983a; Ungerleider and Desimone, 1986; ?; Ship and Zeki, 1989a,b; Movshon and Newsome, 1996). Evidence for direct subcortical inputs from the superior colliculus, the pulvinar and the lateral geniculate nucleus of the thalamus has also been reported (Ungerleider et al., 1984; Fries, 1981; Ffytche, Guy, and Zeki, 1995). 
Area MT is highly specialized for the processing of visual motion information with a proportion of about $90 \%$ of neurons with responses selective for the direction of translational motion (Maunsell and van Essen, 1983b; Felleman and Kaas, 1984; Desimone and Ungerleider, 1986; Logothetis, 1994; Albright and Stoner, 1995). Direction selectivity is evident in Gaussian shaped tuning curves with a standard deviation of about $45^{\circ}$ and amplitudes that are at least three times higher than the Gaussian baseline responses to the non-preferred direction of motion (Logothetis, 1994). In contrast to its afferent cortical areas neurons in area MT are capable to encode pattern motion and thus resolve the aperture problem arising from local ambiguities of motion perception when two superimposed directions move orthogonal to each other (Movshon and Newsome, 1996; Pack and Born, 2001). Moreover, MT neurons integrate motion information in a roughly linear way: When multiple directions of motion are presented within their RFs, the response is typically found to be a scaled average of the response to the component motion directions when presented individually (van Wezel et al., 1996; Recanzone and Wurtz, 1997; Britten and Heuer, 1999; Treue, Hol, and Rauber, 2000). Similar scaling (rather than summation) behavior has also been reported for neurons in striate cortex, inferotemporal cortex and area V4 (Richmond, Wurtz, and Sato, 1983; Dato, 1989; Snowden et al., 1991; Miller, Gochin, and Gross, 1993; Missal and Vogels, 1997; Reynolds, Chelazzi, and Desimone, 1999) $)^{1}$.

In addition to the tuning to the direction of motion many neurons in area MT are also selective for additional aspects of moving stimuli and motion cues including speed, binocular disparity (depth), and also three-dimensional structure-frommotion and surface orientation in depth (Xiang, Marcar, and Raiguel, 1997; Andersen and Bradley, 1998; Perrone and Thiele, 2001; Kourtzi, Buelthoff, and Erb, 2002).

Motion and disparity tuning has been shown to be represented topologically with gradual changes of preferred motion directions/preferred disparity along columnar bands (Albright, 1984; Roy and Komatsu, 1992; De Angelis, Cumming, and Newsome, 1998; De Angelis and Newsome, 1999). This arrangement reveals tuning discontinuities only across, but not within columns. Furthermore, motion selectivity in area MT is only partly dependent on the input projections from striate cortex. MT neurons remain visually responsive and also broadly direction selective, albeit with an increased visual latency, during inactivation of area V1 (Rodman, Gross, and Albright, 1989; Girard and Salin, 1991; Azzopardi et al., 2003). Only

\footnotetext{
${ }^{1}$ It should be noted that deviations from this rule have been reported for RFs within areas higher in the visual processing hierarchy: Responses to pairs of stimuli presented within the confines of a $\mathrm{RF}$ are often dominated exclusively by one stimulus of a pair of stimuli within inferotemporal and prefrontal cortex (e.g. Everling, Tinsley, and Gaffan 2002; Sigala and Logothetis 2002; Freedman et al. 2003). Both areas represent the highest (or latest) stages of the visual processing hierarchy mentioned above.
} 
additional inactivation at the level of the superior colliculi abolish direction selective responses in area MT (Rodman, Gross, and Albright, 1990). Conscious motion perception and motion discrimination ability, on the other hand, has been shown to depend on the integrity of reciprocal projections between area MT and striate cortex (Pascual-Leone and Walsh, 2001; Moore, Rodman, and Gross, 2001).

Functional relevance for motion discrimination

The perceptual relevance of motion processing within area MT has been particularly highlighted by the pioneering studies of Newsome and collegues who showed a strong correspondence of neuronal response strength and perceptual motion sensitivity (Newsome et al., 1989; Britten et al., 1992, 1996; Britten and van Wezel, 1998; Celebrini and Newsome, 1994, 1995). Neuronal responses in area MT gradually increase with increasing direction information in motion displays similar to perceptual performance. Neuronal sensitivity has even been reported to exceed the sensitivity expected from perceptual thresholds, particularly when spiking activity is integrated over long time intervals (e.g. Britten et al. 1992; Dodd et al. 2001; Heuer and Britten 2004). Similarly, motion-direction perception and directions of eye movement can be biased towards the direction coded by neurons within direction columns when locally microstimulated (Salzman and Newsome, 1994; Bair and Koch, 1996a; Ditterich, Mazurek, and Shadlen, 2003). It should be noted that MT activity appears not to be related to perception of all aspects of motion processing (Cook and Maunsell, 2002a; Williams et al., 2003). The particular importance of area MT for motion processing has also been emphasized by various other approaches. Information content conveyed by MT neurons in response to moving patterns has been estimated to be particularly high (about 30bits/sec) (Bair and Koch, 1996b; Buracas et al., 1998).

Consistent with the wealth of accumulated evidence for the functional implications of area MT for motion processing, the sensory responses in this region have been shown to be affected by attention, which will be the subject for the next section (cf. p 13, section 1.2).

\subsubsection{Spatial RF Properties of Area MT}

In addition to the described featural properties of area MT, the spatial RFs of these neurons have also been studied in detail. The spatial extent of neuronal RFs within area MT increases with increasing eccentricity of their centers (Rosa, 1997). Foveal RFs sum input from a roughly circular visual field region of about $0.7^{\circ}$ to $4.1^{\circ}$ (reflecting the intercepts of linear regression models on RF size and eccentricity), with a linear increase of RF size with eccentricity of their centers that follows a slope of the regression line between 0.6 to 1.35 (Gattass and Gross, 1981; Felleman and 
Kaas, 1984; Desimone and Ungerleider, 1986; Rodman, Gross, and Albright, 1989; Tanaka et al., 1993; Raiguel et al., 1995; Xiao, Marcar, and Raiguel, 1997). RFs within area MT are characterized by response profiles that gradually level off from a center position and are well fit by two dimensional Gaussian models (Raiguel et al., 1995; Britten and Heuer, 1999) ${ }^{2}$.

As a rule of thumb, the size of extrafoveal RFs can be described to be as wide in diameter as the eccentricity of its center. The relative differences in the quantitative estimates of the size-eccentricity dependence (see above) are likely due to differences in mapping methods and cell isolation. It should be noted that RF size has been quantified with various methods. Typically RFs are defined as the minimal region of the visual field for which a neuronal response to visual stimulation is obtained that exceeds the spontaneous response level (Martin, 1994) ${ }^{3}$. However, apart from the frequently used minimal response field definition of the RF studies differ widely in quantifying the RF. Major methodological differences concern the kind of visual stimulation and the measure used to define the spatial extent RFs. The following excursion will briefly introduce the main differences because the current project will employ measures of RF estimation.

\section{Methodological consideration: Defining RF size}

Often, light bars of varying orientations are swept across the visual field and the outer borders are delineated for which responses appear subjectively to be above baseline firing. When RFs are quantitatively delineated visual stimuli are typically presented at up to 25 positions at the intersections of a virtual square grid covering the manually estimated RF outline. Responses to stimuli at these positions are than interpolated to obtain an activity surface. The spatial RF outline is then variously defined to extend up to those position with responses below half-maximum response (e.g. Raiguel et al. 1995), or below two standard deviations of the baseline firing rate (e.g. Pettet and Gilbert 1992; Christ and Li 2001). It has been consistently reported that manual approaches underestimate $\mathrm{RF}$ sizes by about $10 \%$ compared to quantitative methods (Raiguel et al., 1995; Chapman and Stone, 1996)). Surface interpolation methods do likewise provide varying RF size estimates: Raiguel and

\footnotetext{
${ }^{2}$ Most MT neurons have excitatory RF activation profiles that follow a two-dimensional Gaussian shape with a center with maximal responsitivity (Raiguel et al., 1995; Lagae et al., 1994a,b; Graziano, Andersen, and Snowden, 1994). However, a subpopulation of area MT cells in the owl monkey ( $17 \%$ of the neurons) has been reported to have RFs with no fixed excitatory RF center (Born and Tootell, 1992; Born, 2000). For these neurons, termed complex motion contrast cells, the stimuli with an optimal size yielding the maximum response can be positioned at different regions within the RF (Born, 2000). In other words, these neurons appear not to summate input with regard to a fixed center of their RFs but dynamically integrate direction information with respect to multiple RF centers constrained only by the size of the stimulus. Neurons with such properties have not been reported within MT of macaque monkeys.

${ }^{3}$ Note that early conceptualizations of RFs already considered silent influences from the RF surround to be part of the RF which are not considered in the most commonly used minimal response field definition (Kuffler, 1953; Barlow, 1953)
} 
colleagues reported that spline fitted RF profiles result in smaller estimates than two-dimensional gauss fits of the spatially distributed responses (Raiguel et al., 1995; Xiao, Marcar, and Raiguel, 1997). Similarly, compared to hand-plotted RFs, the gaussian modelled RFs were found to be on average 2.3 times larger.

In addition to differences in visual stimulation protocols, RF estimates are also variously based on different size measures including (i) the square root of the RF surface (e.g. at half maximum), (ii) the square root of the mean vertical and horizontal width constants, (iii) the sum of the angle radius, or (iv) the distance of two points along the surface which corresponds to the square root of the peak response (e.g. Blatt and Andersen 1990; Barash et al. 1991; Raiguel et al. 1995; Ben Hamed et al. 2001; Eifuku and Wurtz 1999). While all these measures index areal extent the heterogeneity does not ease comparison of RF size estimates across studies. To complicate things further RF size is also infrequently estimated based on responses to stimuli of gradually increasing diameter. The resulting summation profile of activity asymptotes for neurons which do not have an antagonistic surround and begins to drop after a stimulus with the diameter which results in peak responses for neurons with surround (Raiguel et al., 1995; Press et al., 2001). For area MT neurons it has been shown that the estimates of the optimal stimulus radius at which the summation profile asymptotes (and did not fall below $85 \%$ of the peak response) roughly correspond to estimates from mapping RFs with smaller stimuli at 25 positions of a virtual square grid (the ratio of the different size estimates was 0.67 ; cf. Raiguel et al. 1995). In summary, the diversity of RF mapping methods and measures complicates comparisons of existing studies. When comparisons of different methods have been done differences between manual and quantitative methods were obvious, while the estimates from different quantitative approaches differed less to each other. The most widely used approach to measure RF size is the computation of the square root of the visual field area in which responses are above half of the maximum response (half-height $R F$ ) that can be obtained from a neuron when stimulated at its RF center.

\section{Center-surround organization: Local saliency enhancement}

Apart from the excitatory RF, the majority of neurons in area MT exhibit a silent antagonistic surround region, particularly in superficial (i.e. output-) layers II and III (Allman and Miezin, 1985; Tanaka et al., 1986; Born and Tootell, 1992; Born, 2000; Raiguel et al., 1995, 1999; Xiao et al., 1995). Surround influences are not strictly concentric in the majority (80\%) of area MT RFs (Xiao, Marcar, and Raiguel, 1997). The extent of suppressive influences is maximal at a distance of about 1.5 times the RF diameter from the excitatory RF center, while $50 \%$ of the maximum suppression is obtained even at about $3 \mathrm{RF}$ diameters away from the $\mathrm{RF}$ 
center (Raiguel et al., 1995; Xiao, Marcar, and Raiguel, 1997) ${ }^{4}$.

The surround influence is typically silent, i.e. stimuli placed in these regions do not trigger responses by themselves, but suppress responses to visual stimuli applied to the center of the RF when stimulated simultaneously. The surround inhibition is in general stronger when the motion direction and speed of center and surround stimulation becomes similar (Xiao et al., 1995; Xiao, Marcar, and Raiguel, 1997). This observation also reveals that surround inhibition is tuned to the same direction as preferred by the center (Sengpiel and Sen, 1997). As a consequence, visual responses are strongest when stimuli move in the preferred direction within the confines of the RF either in the absence of surround motion, or within a surround of motion in the opposite direction. This antagonistic feature of the center-surround organization of cortical neurons reflects an inherent mechanism to increase the local stimulus (motion) contrast, i.e. the saliency of visual signals.

Center-surround organization within area MT has also been suggested to serve further functions in addition to the bottom-up saliency enhancement of local motion discontinuities, including specific roles in encoding structure from motion, tilt and slant of surfaces derived from motion and depth cues, and the resolution of ambiguous motion (Xiao, Marcar, and Raiguel, 1998; Bradley and Andersen, 1998; Gautama and Van Hulle, 2001; Born and Tootell, 1992; Born, 2000; Duncan, Albright, and Stoner, 2000). These theoretical roles of the antagonistic organization of RF center and surround highlight the potential of properties at the single neuron level to account for perceptual phenomena in visual processing. However, in light of the current thesis, they highlight the potential role of silent, suppressive surround influences to modulate neuronal responses.

\section{Spatial summation and response normalization}

Of particular relevance for the current project is the observation that motion input is integrated by MT RFs spatially in a roughly linear way (Nowlan and Sejnowski, 1995; Britten and Heuer, 1999; Priebe, Churchland, and Lisberger, 2002). Britten and Heuer (1999) recorded the response to small Gabor patches moving in the preferred direction of the neuron. Response strength to a pair of these patches presented at various locations in the RF could be best explained by a scaled summation of the responses to single patches at the respective locations. This finding is thus similar to the scaled average responses observed to different motion directions within the RF (see p. 7). It suggests that the neuronal response strength is determined by a gain control process that acts roughly linearly across the RF and is consistent with

\footnotetext{
${ }^{4}$ Note that earlier studies reported suppressive influences at locations 7-10 RF diameters away from the RF center, similar to recent results from optical imaging of intrinsic signals (Allman and Miezin, 1985; Grinvald et al., 1994; Das and Gilbert, 1995). The most likely source for the discrepancy is a methodological one. In support of the finding from Raiguel and colleagues, electrical stimulation during intracellular recordings in striate cortex results in subthreshold integration fields (in area V1) extending 2.5 to 3.3 times the RF size (Bringuier et al., 1999).
} 
a divisive normalization rule: Rather than merely summing the influence of stimuli at different positions within the RF, the final response does more mimic the result of the division of the sum of all stimulus influences obtained across the RF (e.g. Heeger and Simoncelli 1996; Sclar, Maunsell, and Lennie 1990). Britten and Heuer (1999) proposed that lateral connectivity within area MT is the likely source for such a divisive operation because of the distance of the observed scaling: Effective normalization could be obtained for stimuli placed more than two times the RF diameter away from the RF center stimulus (which extended $20^{\circ}$ for their average RF size of $9^{\circ}$ ), which corresponds to the estimated extent of lateral connections within area MT.

In summary, the RFs of area MT neurons have been characterized functionally and spatially in great detail. This accumulated knowledge provides a firm background to test attentional influences on the spatial RF outline. The following section will survey already known effects of (spatial) attention and link this knowledge from area MT neurons with findings from other areas which are interconnected with area MT. The next section also introduces two influential hypothesis of selective attention which differ in predictions about the existence of changes of RF size concomitant with changes in focused attention. 


\subsection{Attentional Modulation within Visual Cortex}

Selective attention alters the responsiveness of single neurons at all stages of cortical visual information processing tested. Recent progress of the neurophysiology of attention has provided a variety of insights about the working principles of selective attentional modulation (for recent reviews: Treue 2001, 2003; Maunsell and McAdams 2001; Maunsell and Cook 2002; Assad 2003; Pessoa, Kastner, and Ungerleider 2003; Yantis and Serences 2003; Coull 1998). The project of this thesis attempts to add some insights concerning the generality of some of these principles and constrain models of the underlying functional architecture of selective attentional modulation within macaque area MT. More specifically and already introduced in previous sections, the current project investigates the influence of selective spatial attention on the spatial tuning of receptive fields of area MT neurons. The following paragraphs will therefore survey empirical key findings of spatial attention mainly from the neurophysiological domain and introduce two conflicting hypotheses about their neuronal mechanisms. Aspects of attentional modulation with particular relevance for the current project will be highlighted throughout this section.

\subsubsection{Psychophysics of Spatial Attention}

Spatial attention exerts a far reaching influence on the visual representation of our environment. Visual details at locations that are not scrutinized by focal attention are often failed to be recognized (Rensink, O'Regan, and Clark, 1997; O'Regan and Rensink, 1999). One of the core assumptions about attention is therefore the concept of a limited capacity of selective and high resolution processing that can be flexibly allocated to particular regions in space (Kahneman, 1973). In this vein, allocation of attention to a particular region in space has been shown psychophysically to enhance the efficiency of processing visual information at that location which is evident in lower perceptual thresholds, faster detection times, and higher judgment accuracy at attended locations (Posner, Snyder, and Davidson 1980; Cave and Bichot 1999, see below). These empirical findings have given rise to various hypotheses about the mechanisms and psychological working principles of attention. Three aspects of particular relevance for the current study concern (i) the capability of attention to enhance processing relevant signals rather than merely to reduce processing of irrelevant, non-attended signal, (ii) the general finding that attention exerts particularly strong effects when attended targets are embedded in distracting visual information, and (iii) the spatial spread of attentional influences relative to the focus of attention in visual space. These aspects are at the core of controversies in the psychophysical and neurophysiological domain.

With regard to the first two aspects, some accounts of attention state that atten- 
tion primarily reduces the influence of distracting information and thereby enhances perceptual performance (Desimone and Duncan, 1995; Luck et al., 1997a). Accordingly, attention is equated with a filter that reduces noise and interference (e.g. from distracting stimuli, spatial uncertainty, luminance on- and offsets, internal noise in variation of vigilance, etc.). This view has been questioned, however, by various proposals assuming that attention is capable to strengthen, or amplify the representation of visual signals in the absence of interfering signals. Evidence for a role of attention in this latter aspect, signal enhancement, has been established on the grounds of recent psychophysical and human ERP findings (cf. Hillyard, Vogel, and Luck 1998; Blaser and Sperling 1999; Carrasco, Ling, and Read 2004 and discussion, p. 112, section 4.4.2). It should be noted that both perspectives might not be mutually exclusive, because reduced processing of unattended visual information and amplified processing at attended regions could occur simultaneously (which might be one of the reasons of difficulties to dissociate them experimentally). Nonetheless, the emphasis on either signal enhancement, or noise suppression is critical with regard to the neuronal mechanisms thought to underlie attention (see below).

Differential facilitatory and inhibitory effects of attention also play a role in controversies about the spatial distribution of attention. Attending to a particular region in space has been shown to affect processing of stimuli in the vicinity of the focus of attention. Some studies report enhanced perceptual accuracy and reduced response times in the immediate vicinity of the focus of attention (Eriksen and St.James, 1986; LaBerge, 1995). Others have found enhanced interference closer to the focus of attention reflecting a suppressive surround with decreased perceptual sensitivity (Mounts, 2000a; Cutzu and Tsotsos, 2003). While one solution of the empirical discrepancy might lay in the nature of the tasks used to test attentional distribution (cf. discussion, p. 116, section 4.5.1), neurophysiological investigations of the attentional effect on spatial sensitivity profiles of individual neurons can help in understanding the general underlying principle. This is equally true for further attentional influences on the spatial representations inferred from psychophysical studies, including distortions, mislocalizations, and changes in spatial resolution or grain of visual processing with spatial attention. For example, attention has been shown to repel the perceived position of stimuli away from the focus of attention (Suzuki and Cavanagh, 1997). Similarly, localization of flashed stimuli that capture attention automatically, are biased towards more peripheral locations (Tsal and Bareket, 1999; Musseler et al., 1999). On the other hand, close to the focus of attention line length is perceived shorter as the physical stimulus dimension which might suggest a constriction of space (Tsal and Shalev 1996, but see Prinzmetal and Wilson 1997).

The project of the current thesis investigates likely neural correlates of the effects 
of attention on the spatial representation. It touches the intriguing possibility that the discussed perceptual consequences of spatial attention are based on mechanisms evident at the single neuron level. The following sections will survey the known modulatory effects of attention at this level of single cortical neurons after a brief history of this research line.

\subsubsection{A Brief History on Top-Down Modulation}

Visual responses in sensory cortices have long been thought to be determined solely by the nature of the visual stimulus that overlap the neuronal receptive region in space. Since the late seventies extraretinal factors have become evident to influence these sensory responses in a selective way: Early studies recorded responses from parietal cortical neurons and found enhanced response rates to stimuli when they became behaviorally relevant as targets for saccadic eye movements (Mountcastle, 1976; Mountcastle, Andersen, and Motter, 1981; Lynch, Mountcastle, and Talbot, 1977; Robinson, Goldberg, and Stanton, 1978; Yin and Mountcastle, 1978). Reports of such an attentional modulation on motion selective neurons in area MT were one of the first to show this effect for moving stimuli within RFs of neurons that became behaviorally relevant for later smooth pursuit eye movements (Wurtz and Goldberg, 1980; Goldberg and Segraves, 1987; Newsome, Wurtz, and Komatsu, 1988). While these early studies confounded attention towards a stimulus with movement initiation towards that stimulus, which by itself is known to enhance neuronal responses, more recent findings highlight that attention by itself is sufficient to induce changes in response strength of sensory neurons, i.e. also in the absence of the planning of directional movements (Colby, 1996; Colby and Goldberg, 1999; Treue and Maunsell, 1996; Ferrera and Lisberger, 1997; Seidemann and Newsome, 1999; Recanzone and Wurtz, 2000).

Particular progress in elucidating the mechanisms and principles that govern attentional effects within visual cortex has been made within the last decade (for reviews: (Maunsell and McAdams, 2001; Maunsell and Cook, 2002; Treue, 2001, 2003; Assad, 2003; Pessoa, Kastner, and Ungerleider, 2003; Yantis and Serences, 2003)).

\subsubsection{Attentional Gain Modulation \& Sensitivity Enhance- ment}

It has generally been established that spatial attention modulates the sensitivity of neurons within the visual cortex: Attending to an excitatory stimulus inside the RF of neurons typically enhances the strength of neuronal responses. Early studies suggested that this spatial attention effect changes the selectivity of the 
neuronal response and thus is particularly evident for stimuli that correspond closely to the preferred, or most effective, stimulus of the neuron, while less attentional influence was found for non-preferred stimulus features (Haenny and Schiller, 1987; Spitzer, Desimone, and Moran, 1988). More recent data have clarified, however, that attention does not alter the selectivity of neurons but rather changes neuronal responses proportionally by a scaling factor following a multiplicative law (Treue and Martinez-Trujillo, 1999; McAdams and Maunsell, 1999).

\section{Multiplicative gain modulation}

McAdams and Maunsell (1999) analyzed the responses of V4 neurons to different orientations of gratings and observed that attention multiplicatively scales the Gaussian shaped orientation tuning curve by a multiplicative factor of 1.3. Spatial attention acted therefore by increasing the gain of neuronal responses proportionally and multiplicatively and did not change selectivity for orientations, i.e. the tuning width (proportional to the tuning height) remained constant. Extending this finding, Treue and Martinez-Trujillo (1999) found that responses to different directions of motion presented within RFs of area MT and MST neurons are multiplicatively scaled even when attention is directed always to the same location outside the RF. In this experiment, monkeys attended either the preferred or null direction of motion at a location outside the $\mathrm{RF}$ while an irrelevant motion pattern inside the $\mathrm{RF}$ moved in one of twelve directions each trial. The resulting motion tuning curves of the ignored tuning pattern were scaled versions of the sensory tuning function. There was no change in the width, or selectivity, of the tuning function which reflects that a a single gain factor applied to sensory responses is sufficient to account for the observed effects (Treue and Martinez-Trujillo, 1999).

The sign of the attentional scaling varied, however, depending on whether the attended motion pattern moved in a direction similar to the preferred direction of the neuron or not: Enhanced versions of the sensory tuning function were found when the preferred direction was attended, while reduced versions were obtained when the non-preferred direction was attended. This result gave rise to the hypothesis that the strength of attentional modulation observed in single neuronal responses depends on the similarity of the attended feature and the preference of the neuron. According to this feature-similarity gain hypothesis selective attention imposes a unified gain on neuronal responses (Treue and Martinez-Trujillo, 1999). The observed magnitude of attentional modulation would be accordingly dependent only on the overlap of the attended feature, e.g. motion direction, orientation, or spatial location, and the preference of the neuron, being stronger when both are similar.

The proposed gain hypothesis of attention provides a general framework to explain neurophysiological findings of attentional modulation. It is consistent with a variety of attentional phenomena revealed by human electrophysiology (Hillyard, Vo- 
gel, and Luck, 1998), functional MRI (Saenz, Buracas, and Boynton, 2002; O'Murray and Wojciuluk, 2003) and psychophysics (Blaser and Sperling, 1999; Yeshurun and Carrasco, 1999; Carrasco and Penpeci-Talgar, 2000).

\section{Generality of gain modulation}

The observed multiplicative interaction of attention and sensory responses illustrates that attention does not alter the quality, i.e. selectivity, of sensory responses. Apart from attention, the same influence on sensory responses has been reported in various other realms of neuroscience. For example, some area MT neurons are tuned to speed and speed tuning curves increase in gain when the motion-direction of the stimulus is closer to the preferred direction, signifying a gain interaction of speed and direction tuning (Rodman and Albright, 1987). Furthemore, gain effects on neuronal responses have also been described within macaque area $7 \mathrm{a}$ with neuronal responses that vary as a function of a combination of eye and head-movement, or retinal location and position of the eyeball in the orbit (Andersen, Essick, and Siegel, 1985; Brotchie et al., 1995). Moreover, models have utilized gain fields to achieve translation invariant object recognition and context dependent sensory-motor mapping among others (Salinas and Abbott, 1996, 1997; Salinas and Thier, 2000; Salinas and Abbott, 2001; Salinas, 2004). Multiplicative interactions have further been implicated in the emergence of auditory RFs and adaptation effects affecting the spatial summation of area V1 neurons (Pena and Konishi, 2001; De Angelis, Ohzawa, and Freeman, 1995).

To summarize, attentional modulation of neuronal responses is consistent with changes in gain. Gain modulation does not alter neuronal feature selectivity, or feature tuning. The feature-similarity gain hypothesis extends this finding and suggests that attentional gain should not only leave feature tuning unaffected, but also the spatial tuning parameters of neuronal responses (RF size and shape) (Treue and Martinez-Trujillo, 1999). However, this extension to the spatial domain is still not validated empirically. Furthermore, an alternative account of attention suggests that changes in (spatial) tuning are at the core of the mechanisms of attention. This contrasting viewpoint is based on the interpretation of non-multiplicative response modulation with attention to one of two stimuli presented within the RF. The implications of this finding will be surveyed next.

\subsubsection{Push-Pull Effect of Selective Attention: Gain versus Competition}

Attention exerts particular strong influences on detection and discrimination accuracy and response speed in detection tasks when the behavioral relevant stimulus is embedded in distracting visual information. Neurophysiological studies from 
the past two decades have found that this finding is evident at the level of single neuronal RFs within extrastriate visual cortex: It is generally reported that attentional response modulation is particularly large when there are two stimuli presented within the confines of a neuronal RF (rather than one), but only one is attended (Moran and Desimone, 1985; Chelazzi et al., 1993; Luck et al., 1997b; Recanzone and Wurtz, 1999; Treue and Maunsell, 1996; Treue and Martinez-Trujillo, 1999; Treue and Maunsell, 1999; Reynolds, Chelazzi, and Desimone, 1999; Reynolds, Pasternak, and Desimone, 2000). In these studies an effective (preferred) and an ineffective (non-preferred) stimulus is typically placed within the RF. The sensory responses to such a stimulus pair is typically reported to be a scaled average of the responses to the individual stimuli when presented alone (see section 1.1.1, p. 7). With such a stimulus display the push-pull effect (Treue, 2001) of attention is evident in response enhancement if the effective stimulus is attended (push component) and a reduced response strength if the non-effective stimulus is attended (pull component) compared to the sensory response, i.e. in the absence of peripheral attention.

A comprehensive example of this push-pull effect across the whole tuning function has been provided by Treue and Martinez-Trujillo (1999) who recorded responses of area MT neurons while monkeys either attended a random dot pattern within the RF moving in the null direction of the neuron, or a 'tuning pattern' which moved in either of 12 directions of motion. Both stimulus patches were simultaneously presented within the confines of the RF. The authors obtained an enhanced version of the pair-based tuning function when the tuning pattern was attended and a suppressed version when the non-preferred direction of motion was attended.

This finding has given rise to conflicting hypotheses about the neuronal architecture of attention, which either take recourse to a space-based mechanism involving changes in the RF size, or to gain-based explanations as described above. Both of these accounts will be introduced in more detail next.

According to the gain mechanism of attention discussed above, the push-pull effect of attention results in a particular strong attentional modulation simply because attending one stimulus of a pair does not only affect an excitatory response component to a single stimulus, but is also capable to decrease the influence of the effective stimulus when attention is directed to the non-effective stimulus. As a consequence the possible range of response strength that can be modulated is doubled. Consistent with this proposal, attentional modulation of the pair response has been reported to be twice of that obtained for single stimulus displays (Treue and Martinez-Trujillo, 1999). This finding might be explained by the influence of a uniform attentional gain which is turned up when a stimulus preferred by a neuron is attended and turned down if a non-preferred stimulus is attended.

However, the up- and down-regulation of responses with attention to one stimulus 
of a pair might reflect a non-multiplicative modulation of firing rates that is based on a change of the spatial sensitivity of a neuron: Attending to one of two stimuli that are placed within the confines of a single neuronal RF requires that attention can be directed at a smaller spatial scale than the size of these RFs (cf. section 1.2.5, p. 25). The differential up/down regulation of neuronal responses in this situation suggests that attention has a finer spatial resolution than the size of single neurons within extrastriate cortex. This finding might be due to a specific weighting of the spatial sensitivity at the position of the attended stimulus. Such a spatial weighting has been suggested by two divergent hypothesis: The gain hypothesis could be accommodated with a change in spatial tuning when it is assumed that neurons showing push-pull responses integrate responses from presynaptic neurons which have smaller RFs that match more closely the size of the attended stimulus. Spatial attention might multiplicatively modulate responses of these neurons and induce a non-multiplicative, spatially specific weighting of neuronal response at the next higher stage of processing which corresponds to extrastriate cortical neurons which show the push-pull effect of attention (Maunsell and McAdams, 2000, 2001; Treue, 2001).

In contrast to an account based on gain modulation the push-pull effect has originally given rise to an alternative account, which has become known as the biased competition hypothesis, or integrated competition hypothesis of selective attention and was introduced in the psychological domain by Duncan and Humphreys (1989) and transferred to neurophysiology by Desimone and coworkers (Desimone and Duncan, 1995; Desimone, 1998).

Biased competition and RF-shrinkage hypothesis

The biased competition account of selective attention provides a rather general framework of the influence of attention on visual processing. It originated in the pioneering study of Moran and Desimone (1985). The authors reported the first push-pull effect of attention on responses of area V4 neurons while monkeys attended one of two colored oriented bars within the RF. They observed an average reduction of responses of about $200 \%$ when a non-effective, rather than effective, colored bar was attended. This finding led Moran and Desimone (1985) to suggest that

"... the effect of the unattended stimulus is attenuated, almost as if the receptive field has contracted around the attended stimulus."

This RF contraction-, or RF shrinkage-, hypothesis has since been evoked in a multitude of attentional studies to serve as the basic explanation of attentional effects in visual cortex (e.g.Luck et al. 1997a; Reynolds, Chelazzi, and Desimone 1999; Chelazzi, Miller, and Duncan 2001; Christ and Li 2001). It is at the core of 
the biased competition hypothesis of attention. This account states that attention acts by a tonic top-down signal, thought to originate in frontal and parietal areas, on sensory responses coding specific stimulus representations. This bias signal induces an advantage for the attended stimulus representation in the normal ongoing mutual interactions of sensory neuronal responses. At least three major empirical findings are thought to support this account, which will be discussed in turn.

First, the existence of a tonic signal that is selectively enhancing responses of neurons or neuronal populations that overlap the attended stimulus position has been reported in neurophysiological and human imaging experiments during expectation periods and in the absence of the behavioral stimulus (e.g. Luck et al. 1997a; Chelazzi, Duncan, and Miller 1998; Kastner et al. 1999). Such a tonic signal in the expectation of relevant events in the environment corresponds to a top-down bias on sensory responses. However, it should be noted that a selective, tonic top-down signal is also entailed in the gain hypothesis presented above. Second, attention should bias mutual interactions of responses of sensory neurons. This assumption relies on the existence of mutual neuronal interactions in the absence of attention (Kastner et al., 1999; Kastner and Ungerleider, 2000; Reynolds and Desimone, 1999; Pinsk, Doniger, and Kastner, 2004). Proponents of the biased competition hypothesis typically term these interactions "competition" and claim variously that "stimuli", "stimulus representations", "cortical representations", or "neuronal populations" are competing for neuronal resources (e.g. Desimone and Duncan 1995; Reynolds and Desimone 1999; Pessoa, Kastner, and Ungerleider 2003). The nature of the competition is typically left unspecified (see below). However, even though not explicitly stated by any of the proponents it could be equated to what is more specifically treated as response normalization, or gain control. Evidence for gain control can be derived from a large set of studies which report that responses of neurons to pairs of stimuli are not the sum, but a scaled average of the component responses to the individual stimuli (cf. p. 11, section 1.1.2).

According to the hypothesis attention introduces a bias of mutual interactions of neuronal responses that puts emerging representations of the attended stimulus at an advantage over representations of unattended stimuli. This effect is supported by all studies reporting a push-pull influence of attention on neuronal responses (Moran and Desimone, 1985; Treue and Maunsell, 1996; Treue and Martinez-Trujillo, 1999; Luck et al., 1997a; Chelazzi et al., 1993; Kastner et al., 1999; Kastner and Ungerleider, 2000; Kastner et al., 2001; Kastner and Ungerleider, 2001; Reynolds and Desimone, 1999; Rolls and Deco, 2002; Yantis and Serences, 2003). Typically, proponents of the model take recourse to fMRI studies to show that this effect is scaled to the size of RFs rather than on physiological studies like the pioneering study by Moran and Desimone (1985) (Kastner et al., 2001; Kastner and Ungerleider, 
2001; Pessoa, Kastner, and Ungerleider, 2003) ${ }^{5}$. Accordingly, biased competition claims that the push pull effect is always based on shrinking RFs which effectively eliminates, or filters, the influence of the unattended stimulus. It should be explicitly noted that RF shrinkage has never been observed empirically because the RF has never been mapped when attention is directed to one of two stimuli inside the RF: The push-pull effect of attention (see section 1.2.4, p. 17) has been described only for experiments which did not map the RF of neurons to obtain estimates of size and position of the RF while attention is directed to stimuli at different subregions within the RF. It might therefore be based on a scaling of responses in the absence of a change in spatial tuning as would be expected by the alternative gain hypothesis described above.

The third aspect of the biased competition account of attention which is particularly relevant in the current experimental context is the mechanism proposed to underlie RF shrinkage. The RF shrinkage hypothesis emphasizes that attention primarily acts to inhibit neuronal responses pertaining to unattended stimuli. According to Reynolds and Desimone (1999) RF-shrinkage would follow inhibitory interactions of responses of the target neuron (e.g. in area V4 or MT) with responses of afferent neurons with smaller RFs covering only the attended location (e.g. in area V1 or V2). This version of the hypothesis would thus suggest that attention affects inhibitory interneurons to exclusively gate signals from attended location. It should be noted that the delineation of this hypothesis typically leaves this aspect of inhibition underspecified as exemplified by the fact that Reynolds and Desimone (1999) simply left out inhibitory interneurons in their sketch of a model despite the fact that it crucially relies on them. Furthermore, the hypothesis has been modeled in various architectures that derive from the previously described one. For exam-

\footnotetext{
${ }^{5}$ These fMRI studies highlight the fact that mutual suppression (in their terms: stimulus competition) of stimulus representations are evident particularly when stimuli are placed close to each other so as to encompass a region that overlap neuronal RFs (Kastner et al., 2001; Kastner and Ungerleider, 2001). The authors found that when stimuli are placed close together and processed simultaneuously, then response strength is less than when the same stimuli are processed in succession (Kastner, De Weerd, and Desimone, 1998; Kastner et al., 2001; Kastner and Ungerleider, 2001). Reduced response strength was evident in higher extrastriate visual areas V4 and TEO which have RF sizes estimated to overlap the stimulus arrangement in that respective study (Kastner et al., 2001). The authors were also able to show that suppressive interactions became less in area V4 when the stimuli were placed further apart (to $6^{\circ}$ rather than $4^{\circ}$ ) and thus should have been placed in none-overlapping RFs in area V4 while still encompassing single RFs of area TEO where no reduction of the BOLD response was found (Kastner and Ungerleider, 2001). This finding has essentially been shown by a wealth of neurophysiological studies reporting scaled average responses to multiple RF stimuli (cf. p.7, section 1.1.1). Similar to neurophysiological studies the authors were also able to show that selective attention towards one of the stimuli was able to counteract the reduced response when the relevant stimulus is shown simultaneously with distracting stimuli while the effect on attention with sequential presentation was not evident (Kastner, De Weerd, and Desimone, 1998). This finding reflects the push-pull effect of selective attention. What is new, however, is the interpretation based on the BOLD signal that mutual suppressive interactions are not only scaled to the size of individual RFs but that this should be due to shrinkage of RFs of single neurons.
} 
ple, biased competition has been achieved through mutual inhibition of neuronal responses within the target area without taking recourse to interactions with afferent neuronal populations (Deco and Schuermann, 2000; Deco and Zihl, 2001a,b; Rolls and Deco, 2002). It should be noted, however, that the latter computational model has been built in so general terms that it could also be adjusted to rebuild multiplicative effects with the same mechanism (cf. pp. 222 in Rolls and Deco 2002).

To summarize, the push-pull influence of attention on sensory responses has raised conflicting hypotheses about the mechanisms underlying attentional modulation. Both, gain and competition accounts, are capable to explain the main finding in general terms. Moreover, both accounts suggest a special role in the interaction of neurons within extrastriate cortex which have RFs that are larger than the focus of attention to one of a pair of stimuli in their RF with neurons from afferent neuronal populations and having smaller RFs. Gain models predict that neurons in striate areas that match the scale of the attended stimulus should be scaled multiplicatively (Maunsell and McAdams, 2000, 2001; Treue, 2001). The biased competition hypothesis, on the other hand, suggests in its most widespread formulation that afferent neurons coding irrelevant stimuli are inhibited (Reynolds and Desimone, 1999). Therefore, both approaches assume an effect of attention on neurons with smaller RFs that project onto those neurons showing the push-pull effect.

The following sections will survey evidence of such input modulation as a source of attentional effects observed in extrastriate cortex. Thereafter, insights into the neurophysiology of spatial attention within other brain areas will be discussed in order to provide a more comprehensive background about attentional mechanisms.

\subsubsection{Sites of Attentional Modulation: Input Modulation, Hierarchical Gradient \& Adjustable Spotlight}

The push-pull effect illustrates that the spatial specificity, or resolution, of attention can be finer than the size of the neuronal RFs in areas V4 and MT: Responses of neuronal RFs stimulated with a pair of stimuli are up-, or down- regulated depending on which of the stimuli is attended. This finding has suggested to both, gain and competition, accounts that attention acts on the afferent input of the investigated neurons. Afferent neurons in areas projecting to area V4 and MT, such as V1, V2, and V3 do have a narrower spatial resolution by means of their smaller RFs (see p. 8). Recent empirical evidence directly suggests that attentional modulation in extrastriate areas is due to effects on their input rather than their response stage and thus constrains the mechanistic architecture of attentional modulation.

Attention affects sensory gain

The evidence comes from studies showing that effects of attention resemble changes 
of the physical contrast of a stimulus (Reynolds, Pasternak, and Desimone, 2000; Reynolds and Desimone, 2003; Martinez-Trujillo and Treue, 2002). Responses to stimuli of increasing contrast are known to result in a contrast-response function of sigmoidal shape, reflecting a nonlinear modulation of responses (Tolhurst, 1973; Dean, 1991; Albrecht and Hamilton, 1982; Sclar and Freeman, 1982). Recent studies tested whether attention modulates this sigmoidal function by enhancing the responses at all contrast levels, or whether it effectively increases contrast sensitivity and thus affects mainly intermediate contrasts. Results from area V4 and MT both report the latter effect (Reynolds, Pasternak, and Desimone, 2000; Reynolds and Desimone, 2003; Martinez-Trujillo and Treue, 2002): Attention changes the apparent contrast as is evident in a rightward shift of the sigmoidal contrast-response curve. In other words, contrast-response function and attentional modulation share a nonlinearity: Attention affects neuronal responses particularly strong at intermediate levels of contrast where the contrast-response function is particularly steep. Less modulation is observed at low or high stimulus contrast.

This finding implicates that attention affects the input, rather than the response, of the investigated neurons. If attention would target the response stage of these neurons, independent of the physical contrast of the stimuli, it should have affected the response height (or response gain) to stimuli at different contrast levels to a similar amount. Such a general push, or pull, influence would have shifted the contrast-response function up, or down, rather then rightward (Martinez-Trujillo and Treue, 2002). In sum, attention towards a stimulus acts almost indistinguishable from increases of the physical salience (i.e. contrast) of a stimulus. This suggests that the site of modulation is on the input of the extrastriate neurons.

\section{Hierarchical gradient of attentional modulation}

An influence of attention on the input of area MT or V4 neurons is also indicated by studies showing attentional effects directly on neuronal responses within their afferent areas V1 and V2 (e.g. Motter 1993; Roelfsema and Lamme 1998; McAdams and Maunsell 1999; Vidyasagar 1998; Ito and Gilbert 1999; Christ and Li 2001). This finding does not resolve whether the site of attentional modulation is on the neuronal responses of these earlier areas, or on the spatial integration stage within higher areas. However, it highlights the possibility that attentional modulation already affects early response stages, which are then capable to propagate selectively modulated signals across areas. Consistent with this proposal, it has been shown that the magnitude of attentional effects increase across successive areas along the visual processing hierarchy (Maunsell and Ferrera, 1995; Maunsell, 1995; Maunsell and Cook, 2002).

This hierarchical gradient of the magnitude of attentional effects is derived from studies deploying similar task requirements while obtaining neuronal responses from 
different visual areas (Motter, 1993; Treue and Maunsell, 1996; Treue and MartinezTrujillo, 1999; Treue and Maunsell, 1999; Luck et al., 1997a; Reynolds, Chelazzi, and Desimone, 1999; Cook and Maunsell, 2002a; Williams et al., 2003), or BOLD responses of functional MR scans of the human visual cortex (e.g. Tootell et al. 1998; Kastner et al. 2001; Kastner, De Weerd, and Desimone 1998; Watanabe et al. 1998; Martinez et al. 1999; Gandhi and Heeger 1999; Smith and Singh 2000; Ress and Backus 2000; Sasaki et al. 2001; O'Connor, Fukui, and Pinsk 2002; Müller et al. 2003; Pinsk, Doniger, and Kastner 2004; McMains and Somers 2004). In all these studies attentional modulation was stronger in areas higher in the visual processing hierarchy. This general finding reflects an increase in the selectivity of visual responses as one moves up along the hierarchy. In addition to this hierarchical attentional gradient responses become also more related to perceptual performance as one moves up the visual processing hierarchy (e.g. Leopold and Logothetis 1999; Hochstein and Ahissar 2002; Cook and Maunsell 2002a; Williams et al. 2003).

The influence of task requirements and demand However, the hierarchical gradient of attentional modulation can not only be accounted for by a repeated propagation of attentionally modulated responses at each processing area. Maunsell and Cook (2002) suggested that the strength of attentional modulation is also affected by the precise processing requirements imposed by the attentional task: The authors showed that attention changes the relationship of neuronal responses and behavioral performance differently in two areas of the dorsal processing stream (Cook and Maunsell 2002a, see also Williams et al. 2003). In particular, response changes within the ventral intraparietal area (lying further up in the visual hierarchy than area MT) were shown to be stronger than expected from behavioral performance while attentional modulation of area MT neurons were less than expected from changes of behavioral thresholds in detecting coherent motion in moving random dot patterns (Cook and Maunsell, 2002a).

It should be noted, however, that variations in task requirements are typically accompanied by changes in task difficulty which are known to affect the strength of attentional modulation neurophysiologically and psychophysically (Spitzer and Richmond, 1991; Lavie and Tsal, 1994). The influence of task demand as well as processing requirements and attentional modulation can also be illustrated by a comparison of the strength of effects reported across different studies on directional selective neurons within area MT: The strongest modulation reported has been observed with a task requiring the continuous monitoring of motion direction in order to detect a slight deviation of the speed or direction of motion (Treue and Maunsell, 1996, 1999; Treue and Martinez-Trujillo, 1999). A lower attentional effect on sensory responses has been observed with a discrimination task requiring monkeys to indicate one of two possible directions of motion by making a saccade in the detected direction 
(Seidemann and Newsome, 1999). Even lower, or no, modulation has been reported for delayed match-to-sample tasks (Seidemann, Zohary, and Newsome, 1998; Ferrera, Rudolphs, and Maunsell, 1994) and tasks involving attention to single moving dots in order to pursue it after a delay (Recanzone and Wurtz, 2000; Ferrera and Lisberger, 1997). While there are likely further aspects influencing the different outcomes of these studies, the provided brief overview might illustrate that decreasing task requirements (associated with lower processing demands) go along with a decrease of observed attentional effects.

\section{Flexible adjustment of the attentional scale}

The site and strength of attentional modulation is likely influenced by an additional, spatial aspects: The strength of attentional modulation will vary depending on the degree of overlap of the size of individual RFs and the extent of the focus of attention. Attentional modulation might be expected to occur in areas with RFs with the best match in size with the requirement imposed by the attended target stimuli. As a consequence the earliest attentional modulation could be expected to occur in those visual areas which do not only provide the task relevant feature selective neurons, but which has the best spatial overlap of RF size and focus of attention.

This proposal can be inferred from the psychophysical concept of spatial attention as a spotlight, or a zoom lens, pointing to behaviorally relevant regions in space (Posner, Snyder, and Davidson, 1980; Eriksen and St.James, 1986). A key feature of these metaphors is the flexible adjustment of the spatial scale of the attentional focus with regard to the expected extent of behavioral relevant regions in space. Psychological studies have shown a flexible allocation of attention to different spatial scales. Expecting stimuli at a particular scale enhances processing (in terms of detection speed) of stimuli at the expected size, and degrade perceptual detection speed for larger or smaller sized stimuli (Cave and Kosslyn, 1989). Similarly, attention spread to a relatively broad spatial extent decreases the efficiency of detecting unexpected events at a narrow range, while a narrow focus has the opposite effect (LaBerge, 1983; LaBerge and Brown, 1989). Cueing studies likewise showed that cueing a small spatial region enhances stimulus detection particularly close to the cued position (Eriksen and St.James, 1986; Castiello and Umilta, 1990). These findings suggest an attentional gradient distributed according to the spatial extent which is expected to contain relevant target stimuli (LaBerge, 1995; Cave and Bichot, 1999). Consistent with a flexible extent of the attentional gradient, a recent fMRI study reported a narrower spread of cortical activation in response to cues directing attention to a narrow spatial region compared to cues inducing a broad attentional focus (Müller et al., 2003).

As a constraint to the adjustability of the attentional spotlight, a recent set of studies Yeshurun and Carrasco $(1998,2000)$ have provided compelling psychophysi- 
cal evidence that the attentional benefit is limited by the size of RFs that is required to process stimulus features. Attention even deteriorated performance for stimuli that are presented at eccentricities where neuronal RFs are too small to resolve the featural (or spatial frequency) content of these stimuli. As such, these findings might be taken to reveal that the attentional zooming capability is either limited in absolute terms, or highly dependent on the stimuli that need to be selected by attention. One psychophysical approach has suggested the former, i.e. that the grain or spatial resolution of attentional selection is limited in absolute terms and exceeds the resolution (size) of striate cortical RFs by more than two times (cf. e.g. Intriligator and Cavanagh (2001)).

In summary, attention does affect neurons already early in the visual processing hierarchy. Moreover, attentional effects on contrast in extrastriate areas are consistent with modulation of afferent activity from striate cortex. This finding raises questions regarding the nature of the propagation of selected sensory signals along the visual hierarchy. Parallel to this hierarchy attentional modulation strength increases which has been suggested to be due to (i) specific task requirements, (ii) the overall demand imposed by the task, (iii) the scale or grain of the attentional focus induced by the attentional task, and (iv) the match of the complexity of attended stimulus features and the tuning properties of neurons in different visual areas.

\subsubsection{Cortical Representation of Spatial Saliency}

Spatial attention effects in extrastriate areas MT and V4, and in their antecedent areas $(\mathrm{V} 1, \mathrm{~V} 2)$ reflects the representation of salient locations by means of their spatially defined sensitivity profile: RFs in these regions are organized in retinotopic coordinates. In this way, the implicated visual areas achieve spatial selectivity implicitly (for a theoretical framework cf. Li 2002). On a larger scale this has been nicely demonstrated by fMRI studies showing a spatial retinotopy of attention effects that corresponds to the sensory retinotopy (e.g. Tootell et al. 1998; Somers, Dale, and Seifert 1999; Brefcynski and DeYoe 1999; Martinez et al. 1999, 2001; Sasaki et al. 2001; McMains and Somers 2004). Attentional effects corresponding to the retinotopically mapped region have also been found during the expectation (cueing) phase when no stimulus was shown (Kastner, De Weerd, and Desimone, 1998; Müller et al., 2003). However, such an implicit indication about which location contains relevant stimuli introduces spatial ambiguities when two stimuli are presented within the RF of a single neuron and only one of them is attended (see section 1.2.4 and Luck et al. 1997b). In this vein, a neuron in area MT or V4 might indicate that one stimulus of a pair inside the RF is attended (via enhancement or suppression of responses), but it is not capable to indicate simultaneously the position of that 
stimulus compared to the other stimulus. The ambiguity of spatial coding might even be exaggerated when RFs would shrink and shift depending on which stimulus is attended (see section 1.2.4, p. 19). Recent findings about attentional coding within the intraparietal lobule suggest that one solution to this ambiguity problem might lie in the explicit representation of spatial salience (Gottlieb, 2002).

Models of spatial attention most commonly evoke an explicit representation of spatial salience separate from the coding of behavioral relevant stimulus properties (e.g. Treisman and Gelade 1980; Koch and Ullman 1985; Niebur and Koch 1998; Mozer and Sitton 1998; Itti and Koch 2001; Gottlieb 2002; Pouget and Sejnowski 1997; Behrmann and Haimson 1999). An example of the central theoretical role of an explicit spatial saliency map is the feature integration theory, which assumes that selection of a relevant object depends obligatorily on the encoding of the respective spatial location of that object (Treisman and Gelade, 1980; Treisman, 1998). Based on the assumption that features (color, motion, texture, etc.) are encoded in rather independent feature maps, peaks within a spatial salience map allow to bind signals related to features of an attended object, while simultaneously excluding feature attributes belonging to irrelevant objects (Treisman, 1998).

\section{Spatial attention within the intraparietal lobule}

A neuronal correlate of a spatial salience map can be found in functional properties of neurons within the intraparietal lobule (IPL) including the lateral intraparietal area (area LIP) and area 7a (for recent reviews cf. Gottlieb 2002; Yantis and Serences 2003). IPL neurons respond vigorously to stimuli made salient by abrupt onsets (Yantis, 1998) while there is a rapid reduction of response strength to continuously presented stimuli or frequently repeated and thus non-informative stimuli (Steinmetz, Connor, and Constantinidis, 1994; Steinmetz and Constantinidis, 1995; Robinson, Bowman, and Stanton, 1995; Gottlieb, Kusunoki, and Goldberg, 1998; Platt and Glimcher, 1998; Powell and Goldberg, 2000; Constantinidis and Steinmetz, 2001b). The reduction of responsiveness on a short time scale has not been observed in other extrastriate areas (cf. e.g. Motter 1993). Most notably, neurons with low firing rates to a continuously presented stimulus start firing vigorously when spatial attention is directed voluntarily towards that stimulus (Gottlieb, Kusunoki, and Goldberg, 1998). These saliency responses are largely independent of the properties (features) of the stimulus and thus fullfill a major criterion to be considered as a 'pure' spatial response (Gottlieb, Kusunoki, and Goldberg, 1998; Kusunoki, Gottlieb, and Goldberg, 2000; Constantinidis and Steinmetz, 2001b; Powell and Goldberg, 2000). Furthermore, the salience representation exists independent of movement (saccade-) related, or intentional processes, which are also reflected within IPL (for reviews on motor intention related activity cf. Rizzolatti et al. 1987; Andersen 1995; Andersen, Snyder, and Bradley 1997). For example, Gottlieb and Goldberg 
(1999) observed that most neurons within area LIP encoded the position of the relevant stimulus in an antisaccade task, while only a smaller subset signalled the saccade direction as well, and none did exclusively report saccade direction independent of the behaviorally relevant stimulus location.

Salience-related responses within the IPL reflect further and more general aspects of attentional processes which have not been found in other extrastriate areas within the dorsal or ventral visual pathway: Subpopulations of neurons show shortlatency enhanced responses to spatial instruction cues rather than only to targets presented after instructional top-down information has been provided. Cue related response modulation has been observed in diverse variations of delayed saccade tasks (Colby, 1996; Gottlieb, Kusunoki, and Goldberg, 1998; Gottlieb and Goldberg, 1999), delayed match-to-sample (Constantinidis and Steinmetz, 2001a), and motion discrimination tasks (Shadlen and Newsome, 2001; Eskandar and Assad, 2002).

Intriguingly, human neuroimaging studies point similarly towards a more general role of the intraparietal cortex for shifts of the spatial focus of attention in the context of a fronto-parietal network (for reviews: Mountcastle et al. 1987; Corbetta 1998; Corbetta and Shulman 1998; Mesulam 1999; Gottlieb 2002; Assad 2003; Pessoa, Kastner, and Ungerleider 2003; Yantis and Serences 2003). ${ }^{6}$.

Spatial attention within the frontal eye field

Functional properties that point towards a function similar to the IPL are reported also for dorsal prefrontal areas, particularly the frontal eye fields (FEF) (Corbetta, 1998; Mesulam, 1999). Apart from a widespread involvement in coding motor and visuomotor aspects, neurons within the FEF have been found to signal the position of a behaviorally relevant target in visual search tasks irrespective of their color or shape (Schall and Hanes, 1993). This study reports enhanced neuronal responses in the FEF when the target of a visual search task is placed in the RF, while responses to distractors dropped after a brief transient on-response. The response reduction to the distractors became stronger when the target was placed further away from the RF consistent with the idea of a spatial gradient of activity centered on the target location. In particular, Schall and Hanes (1993) reported an attentional gradient that is reminiscent of psychophysically observed perceptual performance: Various psychophysical studies report a gradual decline of perceptual performance from the center of focused attention (Downing and Pinker, 1985; Henderson and Macquistan, 1993; LaBerge, 1983; LaBerge et al., 1997; Handy, Klingstone, and

\footnotetext{
${ }^{6}$ Furthermore, functional data from human cortices recently have begun to associate intraparietal activation in conjunction with superior parietal regions with shifts of attention not only across space but also across features and objects (Wojciulik and Kanwisher, 1999; Eskandar and Assad, 1999; Kanwisher and Wojciuluk, 2000; Culham and Kanwisher, 2001; Corbetta and Shulman, 2002).
} 
Mangun, 1996). The observation of such a gradient in single neuron activity of the FEF could therefore provide a neural correlate of this behavioral effect (cf. p. 116, section 4.5.1).

In addition to the gradient effect of attention on single neurons in the FEF, a recent set of studies provides more direct evidence for a functional implication of spatial FEF signals to affect behavior (Moore and Fallah, 2001, 2004; Moore and Armstrong, 2003; Gardner and Lisberger, 2002). Microstimulating neurons in FEF can elicit saccades toward positions of the movement fields (MFs) of the neurons. Moore and Fallah (2001) tested whether microstimulation below saccade threshold of these neurons decreased the behavioral threshold to detect luminance changes of a behavioral target within the MFs of these neurons in the absence of the execution of saccades. They found increased behavioral sensitivity to luminance changes when targets were presented within the MFs of these neurons, rather than outside them, in blocks of trials with microstimulation. The thresholds in these blocks were as high as the sensitivity in blocks of trials with spatial attention but without microstimulation (cf. Moore, Armstrong, and Fallah 2003). The spatially selective influence of FEF microstimulation on visual processing has also been revealed in response enhancement observed in extrastriate visual areas. This finding comes from a study which required monkeys to selectively attend one of two stimuli while simultaneously FEF neurons were microstimulated below threshold and activity was recorded in area V4 neurons. When the MF of the microstimulated FEF neurons overlapped with the $\mathrm{V} 4 \mathrm{RF}$, the authors reported of a response enhancement, while visual responses to stimuli outside the MF resulted in a moderate response decrement (Moore and Armstrong, 2003). Thus, the influence of FEF microstimulation on V4 responses resembles the influence of spatial attention on neuronal responses in area V4 (McAdams and Maunsell, 1999; Reynolds, Chelazzi, and Desimone, 1999).

In summary, spatial attention is typically conceptualized as an explicit spatial saliency map with peak responses at behaviorally relevant positions within the map. A core assumption of spatial salience coding is its independence of the particular feature dimension that is presented at particular regions in space. A neural correlate of explicit spatial saliency has been found in the intraparietal cortex and the frontal eye field, which constitute parts of a more general fronto-parietal network implicated not only in the representation, but also in the control of the focus of attention.

\subsubsection{Beyond Space as the Unit of Selection: Feature- and Object-Based Attention}

The previous sections dealt with the putative relevance of spatial location as the major unit that is selected by attention: Knowing the location of a behavioral stim- 
ulus in advance enhances perceptual processing and modulate responses of neurons with RFs that overlap with the attended spatial region. The majority of attentional models, including all concepts of a spatial saliency map, assign space a unique role in attentional processing: Spatial selection is commonly assumed to be a necessary presupposition for attentional processing of feature information, or to provide the critical binding function for rather independent featural information (which is true for all models incorporating explicit saliency maps) (Treisman, 1998; Cave and Bichot, 1999).

However, psychophysical and neurophysiological studies suggest that selective processing advantages can be based on featural information on its own (for reviews cf. Cave and Bichot 1999; Olson 2001; Scholl 2001). The major psychological evidence is usually derived from studies showing that particular features (e.g. color or form) are detected faster when they pertain to the same cued object, rather than to a different object, even though the objects are spatially superimposed (Duncan, 1984). Human ERP and fMRI studies likewise showed enhanced activation for visual features that are not directly attended but part of an attended object (e.g. Schoenfeld et al. 2003; Liu et al. 2003), attended objects or faces at identical positions (e.g. O'Craven and Downing 1999), and for attended visual feature dimensions (such as motion direction) irrespective of the spatial position (e.g. Beauchamp, Cox, and DeYoe 1997; Saenz, Buracas, and Boynton 2002). These findings of feature-based selection highlights the potential of featural information to influence processing in functionally specialized visual areas. However, they do not rule out a predominance of space as the unit of selection: Lavie and Driver (1996) show psychophysically that spatial information is used to select relevant objects if they are spatially separated. In this study attention increased accuracy for the cued location and reduced, or even abolished, the influence of object-based factors when they spatially cued one side of an object (Lavie and Driver, 1996).

Apart from the putative predominance of space as the unit of attentional selection, neurophysiological studies have begun to highlight the neuronal consequences of feature-based influences (Motter, 1993; Treue and Martinez-Trujillo, 1999; McAdams and Maunsell, 2000). Moreover, the influence of space and feature information for attentional modulation has been shown to act additively and thus independently on neuronal responses in a study which allowed to disentangle the relative influence of each aspect (Treue and Martinez-Trujillo, 1999; Treue, 2001). In other words, single neurons are affected by featural - in addition to spatial - information when the respective cues are made available in an attentional task. Feature-based influences - in addition to space - should therefore be taken into account in all studies of selective attention. 


\subsubsection{Summary}

This section surveyed the current knowledge of the nature of attentional influences on the firing rates of single neurons. It has been shown that attention does not affect the selectivity of feature tuning but rather scales responses as would be expected by a gain modulation based on a multiplicative rule. This finding also accounts for the push-pull effect of attention which is observed in extrastriate neurons when one of two stimuli within a RF is attended. Alternatively the push-pull effect could be based on a spatial mechanism like RF shrinkage which is supposed by the biased competition hypothesis of attention. Both accounts implicate attentional effects on afferent neuronal populations projecting to extrastriate visual cortex which is supported empirically.

In addition to attentional effects in extrastriate areas with retino-centered RFs (like in area MT or V4) spatial saliency has also been conceptualized as an explicit spatial map. Neural correlates of such an explicit spatial representations are found in parietal and frontal areas. These areas are additionally implicated in the guidance of the focus of attention based on relevant spatial locations as well as featural information. Both, space and feature-based attentional influences are suggested to act independently and with similar mechanisms, by the feature-similarity gain hypothesis of attention (Treue and Martinez-Trujillo, 1999). While this hypothesis provides a unified framework for attentional effects on sensory neuronal responses, it is contrasted by the RF shrinkage hypothesis of attention. Before introducing the experimental approach that tests these alternatives, the following section introduces empirical findings about the degree of plasticity of cortical RFs and and the possible role of changes in spatial sensitivity profiles (RFs) for the selective representation of behavioral relevant visual stimuli. 


\subsection{Dynamic Plasticity of Spatial RF Tuning}

Visual RFs are far more than passive devices to transform retinal visual input according to fixed connectivity rules into some output. The spatial outline of RFs varies depending on the context of visual stimulation and on the state of the cortex (for reviews, e.g. De Angelis, Ohzawa, and Freeman 1995; Polat 1999; Gilbert, Ito, and Kapadia 2000; Wörgötter and Eysel 2000). In recent years, various approaches have revealed that the spatial sensitivity profile can be highly dynamic and dependent at any point in time on the activation of its converging input patterns that arise from afferent and intra-areal connected neurons or from feedback influences from higher order areas.

However, plasticity of cortical RFs have rarely been implicated in selective aspects of visual information processing. This section attempts therefore to survey findings about attentional modulation of spatial tuning. Due to the scarcity of data on influences of selective attention the survey will be extended to reports of nonspecific changes of spatial tuning related to motor intention, arousal, learning and reorganization. This survey primarily aims at providing a background to evaluate the role of attention in spatial routing of visual information as might be evident in single neuronal RFs.

\subsubsection{Selective Attention and Spatial Tuning}

A systematic influence of spatial attention on the spatial profile of RFs has up to now been reported only once (Connor et al., 1996, 1997). Connor et al. (1997) recorded stronger responses of area V4 neurons to irrelevant bars when they were presented near an attended target ring rather than near irrelevant rings. Ring stimuli were always placed outside the RF in a circular arrangement, while the irrelevant bars were presented at five/seven positions along one axis of the RF and extending 1.0/1.5 times the RF diameter. Based on this coarse one dimensional RF mapping, the authors found that the summed response for the two/three bars near the attended ring resulted on average in 16/26\% stronger responses compared to the summed response obtained for the more distant bars (Connor et al., 1997). The observed spatial effect was also evident in a shift of the peak response position and was reliably obtained when only a single bar was presented throughout one trial. The study by Connor et al. (1997) thus demonstrates a shift in spatial responsitivity of RF towards the focus of attention. However, due to the fact that attention was always directed outside the RF, the study does not bear on the RF shrinkage hypothesis discussed in the previous section.

The authors convincingly ruled out confounding influences of eye position, and biases in stimulus arrangement relative to the RF center. However, their stimulus 
regime inherently implied that task difficulty also varied with the position of the attentional focus, which could have systematically biased their results despite their on average low error rate ( $1.5 \%$ ). More specifically, (i) some target ring positions were always closer to the fovea and thus likely easier to attend, and (ii) trials with probe bars (1-3 per trial) presented closer to the attended target ring were presumably more difficult than trials with bars flashed at more distant locations. Since task difficulty is associated with changes in arousal and general response strength, it might well have introduced a response bias even though this aspect would not affect the reported directional effect. Connor et al. (1997) also attempted to rule out a systematic influence of asymmetric surround inhibition of the RFs for the spatial response shift in the direction of target rings presented within the surround of receptive field ${ }^{7}$. However, the study did not incorporate a condition to estimate the influence of surround inhibition at the different ring locations on responses to bars presented in the center. While this aspect does not question the overall directional nature of their attentional effect, it does not exclude surround mechanisms as a central source of their results.

Recently a further study reported RF size changes related to selective attention during a target detection task in inferotemporal (IT) neurons. In this study freeviewing monkeys were required to detect and touch a target object on the screen in the presence of one distractor presented either on a plain background or within a complex natural scene (Rolls, Aggelopoulos, and Zheng, 2003). The target could be either effective or non-effective in driving the neuron. The authors report neuronal responses during brief fixation periods as a function of the distance of the effective object to the fovea. Their data show a more rapidly decline of responses with the distance of fovea-to-effective-stimulus when searching the non-effective rather than the effective target on a plain background. No distance dependent effect was found for search in complex natural scene. The authors claim that this result reflects a shrinkage of RFs ( $R F$ size defined as the distance to half maximum response) reflecting a stronger influence of foveal stimuli on inferotemporal neurons (Rolls, Aggelopoulos, and Zheng, 2003). Considering this result as reliable it would reveal the first quantitative demonstration of shrinking RFs when a non-preferred object is attended in the presence of a preferred object as originally hypothesized by Moran and Desimone (1985). However, the shrinkage result suffers from various methodological problems.

In particular, Rolls, Aggelopoulos, and Zheng (2003) did report response rates

\footnotetext{
${ }^{7}$ Note that Connor et al. (1997) reported a lack of correlation of the shift effect with responses to small RF mapping bars at the position of the later target rings. While this finding might suggest that the spatial sensitivity at the target ring positions are not systematically related to the shift effect, it should be noted that they assesed spatial sensitivity at the target ring positions only in a sensory control condition (in advance of the attention trials), which was clearly not matched in attentional demand with the attention trials.
} 
of brief $100 \mathrm{~ms}$ intervals during fixation intervals always relative to the effective stimulus without control of the monkey gaze (i.e. irrespective of the distance to the non-effective stimulus) and without consideration of the sensory RF size of the neurons. It is however an implicit confound in their study that monkeys will have foveated more often the non-effective stimulus when it was the search target than when it was not the target (and the effective object was the target). This implies that foveated non-effective objects contributed (and by definition lower) firing rate values to their analysis in this condition. Consequently the reported responses to the effective stimulus should be lower in this condition irrespective of the distance of the effective stimulus to the fovea and hence are easily explained by a scaling of the $\mathrm{RF}^{8}$.

Furthermore, the results are based on a comparison of conditions which were run always sequentially in the same order in extensive blocks of trials which were set out to investigate a different objection, namely the influence of complex backgrounds. Together with the small number of neurons $(n=17)$ studied and their large cortical separation (c.f. their Fig. 9) the reported data are not capable to provide a valid conclusion on RF changes of area IT neurons. In support of this interpretation recent studies suggest that IT neurons are rather sensitive to small changes in the retinal position of objects (independent of attentional manipulations) which further question the reported RF-estimates because they are based on mere distance measures, rather than exact positions of the effective object relative to the fovea (e.g. DiCarlo and Maunsell 2003).

\subsubsection{Intention and Attention: RFs during Directional Move- ments}

Modulation of the spatial position of neuronal RFs have been most persuasively shown to occur in relation to saccadic eye movements (see below). Studies of eyemovement related activity typically require monkeys to select a saccadic target at some spatial position with the intention of a directional eye movement to that position. Apart from motor intention the process of target selection inherently implies attentional mechanisms. Early studies have revealed that scanning visual scenes involving sequences of saccades and fixations are guided by regions in space with particular salience and above average information content (Yarbus, 1967). Consistent with this finding, abundant psychophysical evidence has revealed that saccadic eye movements are obligatorily preceded by spatial attention to saccadic target po-

\footnotetext{
${ }^{8} \mathrm{~A}$ further methodological problem in this context is the use of a Wilcoxon rank test for these data which will contain more confounded low values (monkeys foveate the non-effective stimulus) in their non-effective search than in the effective search condition. This statistics is inadequate for the kind of data and biases the result to lower p-values.
} 
sitions (e.g. Bahcall and Kowler 1999). A recent study of neuronal responses within area V4 has provided a likely neuronal correlate of these attentional effects (Mazer and Gallant, 2003). The authors report that the direction of saccades of free viewing monkeys could be predicted by enhanced responses of neurons with RFs centered in the direction of the later saccadic target position (Mazer and Gallant, 2003). Consistent with an attentional rather than motor-based effect the prediction could only be based on the peak response of histograms that were aligned to fixation onset prior to the subsequent saccade rather than aligned to saccade onset itself. Saccade-onset aligned effects on neuronal responses have been found in a multitude of studies and are typically indicative of plans or intentions to move (rather than attention) and are found in the majority of neurons within the intraparietal cortex, the frontal eye fields and the superior colliculus (rather than V4) (Andersen, Snyder, and Bradley, 1997).

\section{Peri-movement predictive remapping}

Neurons within these movement related areas typically respond non-selectively to visual stimuli within their RF, and show enhanced responses when a movement is planned towards that stimulus. A subpopulation of neurons in these areas, including the frontal eye fields, the lateral intraparietal area, and the superior colliculus, completely shift and expand their retinocentric receptive field immediately prior to saccade execution to the location that will be occupied after saccade completion (Duhamel, Colby, and Goldberg, 1992; Walker, Fitzgibbon, and Goldberg, 1995; Umeno and Goldberg, 1997; Kusunoki and Goldberg, 2003). These neuronal RFs appear to anticipate the spatial reference frame that will be established after completion of the saccade. Such predictive remapping of space occurs immediately prior to saccade execution and is thus bound to the intention plus initiation to move the eyes. Despite this close dependence to eye-movement related activity, predictive remapping has also been found in small subpopulations of neurons in extrastiate visual area V3 and V3a (Nakamura and Colby, 2002). This finding is consistent with signal from movement related area reflecting a predictive updating of visual coordinates transfered to visual areas (Nakamura and Colby, 2002). It should be noted that these predictive shifts of RFs have not been accomplished by attentional factors alone: Neither abrupt onset stimuli capturing attention automatically, nor voluntary attention to a stimulus without a saccadic movement have revealed predictive changes of RF position (e.g. Nakamura and Colby 2002; Gottlieb, Kusunoki, and Goldberg 1998).

Similar shifts of the receptive region of intraparietal neurons have also been observed in bimodal visuo-somatosenory neurons during manual exploration. RFs of these neurons have been shown to expand during a reaching task concomitant with the use of a rake tool (Iriki, Tanaka, and Iwamura, 1996). The spatially shifted RFs 
of these bimodal, visuo-somatosenory neurons were always reported to comprise regions of the rake tool. Taken together these results suggest a dynamic shift of receptive regions contingent on the directional intention to move.

Peri-saccadic RFs in area $M T$ and $M S T$

Predictive remapping has not been reported for neurons in area MT and MST within the superior temporal sulcus. However, neuronal responses in these areas are affected by the initiation and execution of saccades: Thiele et al. (2002) report that about a fifth of the neurons selectively reduce responses during saccades, while staying active when saccade execution induces motion of the background scene in the RF passively. The authors link this result to a general decrease in visual sensitivity around the time of saccades ('saccadic suppression') (Kaiser and Lappe, 2004). Recently, the influence of eye movement on the spatial tuning of area MT and MST responses has been investigated at different positions in the visual field (Krekelberg et al., 2003). In this study, monkeys were required to make saccades of 20 degrees extent while bright bars were flashed at either of 6 positions separated by 5 degrees (so that about two or three bars fell within the sensory RFs). While neuronal responses in area MT reliably signalled retinal positions in the absence of saccades, the spatial signal was distorted and fell to chance level when bars were flashed from $100 \mathrm{~ms}$ before to $70 \mathrm{~ms}$ after the saccade (Krekelberg et al., 2003). Similarly to the previously mentioned study, the observed spatial distortion could be related to perceptual insensitivity around the time of saccade. As such, they reveal the remarkable degree of neuronal plasticity within area MT and MST that is related to the intention and execution of eye movements.

\section{Peri-saccadic RF shifts: area $V_{4}$}

Similar to area MT and MST, neuronal responses within the ventral pathway have neither been shown to predictively shift their RFs to coordinates present after a saccade. A recent report of saccade-related visual response profiles of V4 neurons rather suggests that RFs are shifted toward the saccadic target region during the time of saccade execution (Tolias et al., 2001). In that study monkeys had to saccade towards a peripheral target spot after dimming of the central fixation point while an irrelevant probe stimulus was persistently present at a random location of the screen. The authors derived sensory RFs based on the transient on-response to the probe (firing rate from $50-100 \mathrm{~ms}$ after probe onset) and found additional response peaks between -92 and $+17 \mathrm{~ms}$ (mean: $-26 \mathrm{~ms}$ ) relative to the onset of the saccade toward the saccade target. This secondary response was particular strong when the saccadic target was located close to the RF of the neurons. Delineation of the outline of these peri-saccadic RFs showed that they were offset from the sensory RFs toward the saccadic target position by on average of 0.4 times the sensory RF diameter (or 4.3 degrees in absolute terms) (Tolias et al., 2001). 
This result reveals a dynamic shift of sensitivity in response to irrelevant probes towards saccadic target positions. The study suffers, however, from a poor resolution of RF maps: The authors used probe stimuli of a fixed size ( 4 degrees) and a fixed inter-stimulus distance ( 6 degrees), which reduces the quality and reliability of their interpolated RFs. As a consequence the absolute values of the dynamic RF changes should be treated with caution. The authors also claim that peri-saccadic RFs are smaller than sensory RFs (by an average of 2.1 degrees), which they suggest to reflect a temporary decrease in cortical magnification. However, it should be noted that a comparison of peri-saccadic RF size with pre-saccadic sensory RFs is not warranted due to the fact that only the later is affected by luminance and feature onset of the probe stimulus. The reported size change is therefore not indicative of a shrinkage of RF. Tolias et al. (2001) also report that in a time interval from -75 to $0 \mathrm{~ms}$ relative to saccade execution, the response profile was already shifted towards the saccade target. Such a pre-saccadic shift of the structure of sensory RFs is reminiscent on the attentional effect of a spatial gradient reported in V4 neurons by Connor et al. (1997). However, the effect remains bound to motor aspects rather than attentional factors.

In summary, the evidence of attentional influences on the spatial sensitivity profiles is limited to the study of Connor et al. (1997). This study suggests a shift in the spatial profiles of RF towards the attentional focus but does not touch the possibility of a size change with attention. Spatial RF plasticity on a trial-bytrial basis have further provided evidence for a shift of RFs toward saccadic targets (Tolias et al., 2001). However, applying the result of Krekelberg et al. (2003) to the spatial shift reported by Tolias et al. (2001) suggests that such perisaccadic RFs do not carry task relevant information on potential attentional target positions (as suggested by the authors), but rather reflects distortions of sensory processes that are more related to perceptual insensitivity (such as "saccadic suppression").

\subsubsection{Effects of Expectation and Alertness}

Various studies have focused on response modulation that are non-specific to particular stimuli within the visual field but are associated with increased states of arousal or alertness. These non-specific states of enhanced expectation has been shown to affect the spatial summation of cortical neurons, i.e. their RFs.

Consistent with its role in the representation of spatial saliency (cf. p. 26, section 1.2.6), early studies have noted that about half of the neuronal population within the intraparietal sulcus respond to stimuli during states of increased alertness (i.e. attentive fixation versus intertrial idling states) (Mountcastle, Andersen, and Motter, 1981; Mountcastle et al., 1987; Motter and Mountcastle, 1981; Bender 
and Youakim, 2001). More recently the spatial sensitivity profiles of these neurons have been reported to be up-, or down-regulated and slightly shifted foveally during attentive fixation compared to constant fixation periods in intertrial periods (Ben Hamed, Duhamel, and Bremmer, 1997, 2002; Ben Hamed and Duhamel, 2002). This modulation is commonly suggested to be specific to the intraparietal sulcus as similar effects have not been observed in other visual areas including area V1, IT and visual thalamic nuclei (Mountcastle et al., 1987; Richmond, Wurtz, and Sato, 1983; Bender and Youakim, 2001). Accordingly, the intraparietal sulcus has been assumed to represent spatial locations in contexts of behavioral relevance which might serve to re-direct attentional resources to potentially interesting locations (Mountcastle et al., 1987). Such non-specific alertness signals do add to the functional involvement of the intraparietal area in attentional processes.

More recently, non-specific effects of arousal on the extent of the spatial integration have also been reported within striate cortex. In particular, Woergoetter and colleagues (Suder and Wörgötter, 2000; Wörgötter and Eysel, 2000) has suggested that arousal effectively biases the dynamical point spread of cortical activation to be more narrowly distributed. In other words, arousal - reflected in tonic thalamic activation - induces spatially more focused sensory responses via thalamo-cortical suppression (Castro-Alamancos, 2002). Within the visual system Wörgötter et al. (1998) have shown that de-synchronized LGN activity (in the $\gamma$ range) which is associated with enhanced alertness compared to synchronized activity (in the $\alpha$ range) is associated with smaller receptive fields in area V1 of the anaesthetized cat. The authors inferred from their results that states of focused attention should rely on the same mechanism as their observed global EEG fluctuations observed in anaesthetized cats (Suder and Wörgötter, 2000). While their is a large gap in the inference from global EEG during anaesthesia to local focused attention effects in awake-behaving primates, the observed shrinkage of receptive fields would be expected to be present also in response to visual stimuli during selective attention. However, it is currently neither known whether such a shrinkage is observed in responses to attended stimuli, nor whether attention results in local EEG fluctuations similar to those observed by Woergoetter and colleagues.

Furthermore, it has not been demonstrated that desynchronized EEG states are selective rather than only modulatory. In this vein it should be noted that receptive field size has been reported to change depending on the depth of anaesthesia which might question the selectivity of the observed spatial effect (Armstrong-James and George, 1988; Simons et al., 1992). 


\subsubsection{Dynamics at Longer Time Scales: Conditioning, Adap- tation and Context-Dependent Plasticity}

The previous sections surveyed evidence of spatial tuning changes on a trial-bytrial basis within a short-time scale. In contrast, plasticity of the spatial summation characteristics of individual neurons can also be induced at longer time scales. Widespread spatial plasticity has been shown to follow learning, conditioning, adaptation and cortical reorganization (Kaas, 1991; Weinberger, 1995; De Angelis, Ohzawa, and Freeman, 1995; Das, 1997). Some changes observed at such longer time scales have also been implicated to recruit mechanisms which also subserve attentional modulation (Gilbert, Ito, and Kapadia, 2000). In this vein they are particularly relevant to be considered in the current context.

Adaptation, artificial scotoma conditioning and cortical reorganization

The extent of spatial summation of striate cortical neurons is influenced by the history of the stimulation in their RF surround. After stimulation of regions surrounding the classical RF for few minutes (typically 15min.) neurons begin to respond to stimuli presented in the previously silent surround (Pettet and Gilbert, 1992; Volchan and Gilbert, 1995; Das and Gilbert, 1995), even in the absence of center stimulation (De Weerd, Gattass, and Desimone, 1995). The observed spatial effect has been reported to be extensive in some neurons, with spatial summation increasing five times the RF sizes (Pettet and Gilbert, 1992). While this effect of conditioning has originally been proposed to reflect an increase of the RF of these neurons a more recent study has shown that the increased spatial extent of the RFs could be accounted for by an overall enhanced baseline firing rate and sensory excitability, or gain, of these neurons, corresponding to a scaling, rather than an increase, of the RF (De Angelis, Anzai, and Ohzawa, 1995). In particular, the observed gain increase (by a factor of 1.2) followed a multiplicative rule resembling the effect of attention (cf. page 16, section 1.2.3).

The observed spatial gain increase after visual conditioning of these neurons has paved the way for various hypotheses about the underlying neuronal mechanism (Gilbert, 1992, 1995; De Angelis, Ohzawa, and Freeman, 1995; Chapman and Stone, 1996). The general assumptions agreed upon by various authors suggest a decrease of inhibition in the surround of the neuronal integration field that unmask excitatory influences of cortical origin (Chapman and Stone, 1996; Chino, 1997; Volchan and Gilbert, 1995). Decreased inhibition has been suggested to involve an adaptation of the underlying inhibitory circuitry (De Angelis, Ohzawa, and Freeman, 1995) which is reflected in decreased inhibitory GABAergic activity surrounding the conditioned region (Chino, 1997). Consistent with this proposal, antagonizing GABA has been shown to mimic the RF expansion in cortical neurons (e.g. Wang et al. 2002). The 
unmasked excitation, on the other hand, has been suggested to reflect long lateral excitatory connections between columns within an area (De Angelis, Ohzawa, and Freeman, 1995; Gilbert, 1995), while influences from afferent and efferent neurons have also been implicated (Chino, 1997). In order to obtain a multiplicative increase of excitability to visual stimulation of the classical RF, neuronal gain control has been modelled successfully by reduced divisive inputs from the surround (c.f. De Angelis, Ohzawa, and Freeman 1995; Heeger and Simoncelli 1996; Heeger, Gandhi, and Huk 2001; for other inhibitory rules reproducing RF integration and expansion see Xing and Gerstein 1994; Kalarickal and Marshall 1999).

The expansion of RFs observed after extensive stimulation of the RF surround and sparing the RF center resembles the recovery phase of a scotoma induced by retinal lesions (the inducing stimulation has therefore been termed artificial scotoma conditioning) and ibotenic acid cortical lesions (Darian-Smith and Gilbert, 1995; Eysel et al., 1999; Eysel and Schweigart, 1999). The similarity of such an effect which is putatively due to recovery from adaptation with recovery from retinal or cortical lesions causing a scotoma highlights therefore similarities of both mechanisms (for a review cf. Pernberg, Jirmann, and Eysel 1998).

Reports of RF size changes associated with recovery from lesions are not restricted to striate cortex but has likewise been reported for area MT (Wurtz et al., 1990; Sober, Stark, and Yamasaki, 1997). In particular Sober, Stark, and Yamasaki (1997) reported increased spatial summation of area MT neurons after subacute lesions concomitant with an increase in baseline activity. This finding resembles the effects of artificial scotoma conditioning (and thus an unmasking of subthreshold excitatory input). These studies therefore reveal that the underlying neural circuitry within area MT and its afferent areas determine the extent of the spatial summation properties of area MT and already propose specific roles of excitatory and inhibitory connections for the emergence of the spatial layout of RFs.

\section{Sensory conditioning}

Apart from effects of artificial scotoma conditioning or direct induction of scotomas, RF size changes have also been found in the context of classical (stimulus-stimulus) conditioning and operant (stimulus-response) conditioning (for a review cf. Weinberger 1995; Das 1997). In these studies neuronal RFs are stimulated with temporally paired stimuli at various spatial displacement with a timing regime known to trigger short or long term potentiation or depression. In this context simultaneous stimulation of RF center and surround has been reported to induce spatially specific enhanced excitability at or near the co-stimulated surround region, while temporal stimulation offsets of eight milliseconds have been found to shift RF centers concomitant with a shrinkage of the RF (Schweigart and Eysel, 2002; Fu et al., 2002). Similarly to the effects of artificial scotoma conditioning these findings are 
assumed to reveal an unmasking and strengthening of lateral excitatory horizontal connections. In addition, however, effects of spatially specific stimulus pairings have been shown to be correlated with a psychophysically measured bias in localization judgements (Fu et al., 2002). This latter point highlights the dependence of spatial summation characteristics of neurons on context-dependent experience of visual stimulation.

\section{Task- and context-related plasticity}

Extended periods of practice and experience with sensory stimuli within the context of a task is capable to reshape the tuning of single neurons which is typically discussed in terms of perceptual learning (e.g. Logothetis and Sheinberg 1996; Ito and Westheimer 1998; Sengpiel and Stawinski 1999; Wallis and Buelthoff 1999; Miyashita and Hayashi 2000; Sigala and Logothetis 2002; Lee, Yang, and Romero 2002). Observed neuronal tuning changes are rarely reported for spatial summation and almost exclusively focus on visual objects and contextual integration of features. However, insights into experience dependent tuning changes might be used to estimate the degree of plasticity of neuronal responses that is induced by attentive learning of relevant visual stimulus constellations with task context (Salinas, 2004). In this vein some recent studies have shown a change in selectivity of neuronal responses on a trial-by-trial basis due to the learned behavioral context of visual stimuli.

In visual cortex tuning changes has been most persuasively shown for neurons within the parietal cortex: For example, Toth and Assad (2002) trained monkeys to use either the color or the location of an otherwise identical attentional cue instructing monkeys to saccade to a target location. They recorded from intraparietal neurons which do respond to spatial and eye-movement related events. Nonetheless they report that on trials with color as the relevant cue dimension, parietal neurons respond in a color selective fashion, while on no-color cue trials that selectivity was absent. Even though the set of used color was rather small in that study to establish task induced color selectivity in more detail, this result suggests a dynamic change in response selectivity from one trial to the next depending on the specific context and learned behavioral significance.

Studies within striate cortical neurons also suggest that individual neurons might be recruited by varying neural circuitries depending on the task requirements. For example, Christ and Li (2001) trained monkeys on a line bisection task and reported that collinear flanking lines induced a facilitatory response to lines within the RF that was specific to line bisection trials and not evident for trials that did not involve line bisection. Based on these results the authors suggested that contextual interactions involving lateral interconnectivity within striate cortex might have been flexibly adjusted during the training phase. As a result, the respective neurons might call upon different neural circuits depending on the task and stimulus context. 
These results are paralleled by findings in other sensory as well as motor cortices. Within the auditory modality expectation of a target tone learned via aversive conditioning has been shown to cause facilitation (and release of inhibition) that is selective to the target frequency in auditory cortex (Fritz et al., 2003). With regard to the motor domain, rapid task-related adjustment to external force fields have likewise been shown in monkey motor cortex (e.g. Gandolfo et al. (2000)).

The discussed changes reveal a remarkable plasticity of the integration of signals in various cortical areas, although few is currently known about the involved mechanisms and the effects on spatial sensitivity profiles of individual RFs.

In summary, this section surveyed findings on dynamic aspects of cortical RFs. Only one study directly suggests that RFs shift to attended locations while a variety of approaches reveal changes in spatial summation of individual neurons in relation to motor intention, expectation, arousal, conditioning, adaptation and attentive learning. The functional significance of the observed RF changes for a selective representation of attended visual stimuli therefore, remains elusive. Together with the previous section on attentional modulation of sensory responses and its putative influence on spatial summation (RF shrinkage), this section provided background information on potential plasticity and its mechanisms from a variety of seemingly distant neuroscientific realms. The next section will therefore introduce the specific hypothesis underlying the current project. 


\subsection{Summary \& Hypothesis}

The three previous chapters introduced three major physiological aspects of visual information processing. The first section showed that visual processing is based on functionally specialized visual areas that encode increasingly complex visual attributes in a hierarchical processing sequence. The major unit for visual transformation is the spatially circumscribed neuronal RF, which increases in size along the visual pathway. Within the dorsal visual path, area MT constitutes a functionally highly specialized visual area which has been particularly well characterized with regard to anatomical connectivity, feature tuning, spatial summation characteristics and their functional relevance for motion processing.

The second chapter introduced insights of the influence of spatial attention for selective visual processing. One of the key questions of the neurophysiological research of attention concern the mechanisms underlying the push-pull effect of selective attention. Two contrasting hypotheses have been put forward to explain the effect. One hypothesis proposes an attentional gain on the sensory responses of neurons, particularly when the neuronal RFs match the scale of the attentional focus (Maunsell and McAdams, 2001; Treue, 2001). The alternative biased competition account of attention has been built around the assumption that attention acts primarily by shrinking RFs around attended stimuli (Moran and Desimone, 1985; Reynolds and Desimone, 1999).

However, shrinking RFs have never been observed empirically. Only a few studies have investigated the outline of neuronal spatial sensitivity (the RF). The insights from these studies were surveyed in the third chapter and suggest a high degree of plasticity of the spatial summation characteristics of individual cortical neurons. Only one study (Connor et al., 1997) has directly shown that attention has a spatially specific impact on RFs. This study was carried out in area V4 neurons within the ventral processing pathway and tested for effects of attention focused outside the investigated neuronal RFs. It showed a spatial shift of response strength of area V4 neurons towards the attended location. It did not test for a potential shrinkage of RFs when one stimulus inside the RF is attended. Therefore, this study does not contribute directly to the controversies about the push-pull effect of attention and the assumption of shrinking RFs. Moreover, the design deployed by Connor et al. (1997) likely confounded task difficultly and spatial attention and only performed a coarse one-dimensional mapping of RFs.

The current thesis was therefore set up to investigate in more detail the influence of spatial attention on the spatial sensitivity of extrastriate cortical neurons. Two major questions guide the design of the experiment, which will be introduced in the next chapter. 
First, spatial attention might change the spatial sensitivity of neurons in different ways. We investigate whether attention shifts RFs towards the stimulus position that is the focus of attention, or whether it merely scales responses uniformly across the RF leaving position unaffected. In order to answer this question we will map RFs of single neurons when attention is directed to stimuli at different subregions within the RF.

The second aspect is the investigation of the RF-shrinkage hypothesis: Do RFs shrink when attention is directed to a position inside the RF compared to RFs when attention is directed outside the RF? This test requires an attentional condition with visual stimuli inside and outside the $R F$ and a method to obtain quantitative, high resolution maps of the spatial sensitivity of individual RFs.

The following section will introduce the methods used to address these questions. 


\section{Chapter 2}

\section{Materials \& Methods}

We recorded extracellular responses of direction-selective cells in area MT to moving random dot patterns (RDPs) in various experimental conditions in one left hemisphere of an adult male rhesus monkey (Macaca mulatta) who was surgically implanted with a head post, a scleral search coil (Judge, Richmond, and Shu, 1980), and a recording chamber. Surgery was conducted under aseptic conditions with isoflurane anaesthesia. The monkey was held under a water restriction schedule at working days and obtained the majority of fluid from juice and tea reward during the experiment. A minimum amount of $30 \mathrm{ml}$ fluid per $\mathrm{kg}$ weight was ensured regardless of the daily amount of reward obtained during experimental sessions. All procedures and experiments were approved by the animal ethics committee of the district government of Braunschweig, Lower Saxony.

\subsection{Experimental Settings}

\subsubsection{Electrophysiological Specification}

Extracellular recordings were obtained with tungsten microelectrodes (impedance 0.5-1.5 M $\Omega$ ), insulated with parylene or epoxyte (FHC Inc. or Microprobe Inc). Transdural penetrations were done with custom made guide tubes. Single-electrode recording was based on either of two systems with similar filtering and gain regimes but different spike discrimination methods. With the single electrode BAK system, raw signals were amplified in the range of 20000-60000 and filtered at $100 \mathrm{~Hz} / 5 \mathrm{kHz}$ (Low/High-Cut). The second system from Plexon Inc. provided a gain range of up to 32000 with a $150 \mathrm{~Hz} / 5 \mathrm{kHz}$ filtering. About half of the cells were isolated with a window discriminator (BAK Electronics Inc.). The other half of the cells were isolated under computer control with box discrimination, or a template matching algorithm provided by software packages from Plexon Inc. 


\subsubsection{Confirmation of Recording Sites}

To access area MT we used a vertical approach with a recording chamber implanted on the skull over the superior temporal sulcus. Anatomical information from the literature and knowledge from previous experiments (Treue and Maunsell, 1996, 1999) guided the exact stereotactic placement of the chamber of the left hemisphere at $8 \mathrm{~mm}$ posterior to the AP-reference center and $13 \mathrm{~mm}$ lateral from midline. At the beginning of the study several penetrations were done to ensure that electrodes were in appropriate parts of area MT in the superior temporal sulcus. The physiological criteria for cells to be attributed to area MT were (i) directional selectivity to uniform motion with an indexed tuning strength (see below for details) that exceeded tuning strength for spiral motion components (expansion, contraction, rotation) (see below for details) and (ii) receptive fields that were confined to circumscribed regions within the visual field (Gattass and Gross, 1981; Maunsell and van Essen, 1983; Desimone and Ungerleider, 1986; Logothetis, 1994; Graziano, Andersen, and Snowden, 1994). The experiment did not consider units with properties suggestive of other areas than area MT, including (i) RFs spanning large regions of a visual quadrant and including the fovea, (ii) with strong spiral motion tuning, (iii) stronger tuning to oriented bars than to moving RDPs, (iv) bimodal direction tuning curves (indicative of orientation tuning), and (v) eye-movement and fixation related responses.

As already noted we performed the main experiment only on neurons which could be assigned to area MT based on the physiological criteria mentioned above. This assignment can also be based on the spatial topography of the physiological criteria. The following two paragraphs will briefly (i) compare the observed topography of RFs within and surrounding the superior temporal sulcus with that reported in the literature, and (ii) then provide the results of the anatomical reconstruction obtained after recording the main experiment.

\section{Characteristics of RF Topography}

The majority of neurons assigned to area MT had RFs in the lower right visual quadrant, while a small sample of cells had RF centers in the upper right quadrant close to the vertical meridian. The anatomical distribution of these RFs followed nicely the expected topography of area MT. The representation of the lower visual field has been described to be at the posterolateral part of area MT, while the upper visual field representation is reported to lay anteromedially (Gattass and Gross, 1981; van Essen and Maunsell, 1983; Desimone and Ungerleider, 1986). This is in general agreement with the observed distribution of RF centers. Furthermore, neuronal response properties surrounding the area where most neurons were sampled for the experiment matched reports from the literature: We found large neuronal RFs crossing the vertical meridian antero-lateral to our recording site (Desimone and Ungerleider, 1986). These neurons could be located in area MST, which lies 
lateral-to-anterolateral to MT and whose neurons have larger RFs that typically include the fovea and are responsive to more complex optical flow patterns ('spiral motion', see Graziano, Andersen, and Snowden 1994) such as expansion and rotation (Desimone and Ungerleider, 1986). We tested neurons with such spiral motion at two different speeds and found RFs tuned to spiral motion (expansion, contraction, or rotation) as expected for area MST antero-laterally to our main recording site. Furthermore, we recorded neurons with properties expected for area V4/V4t at the posterior border and posterior-lateral to our main recording site. At these sites the majority of neurons were orientation selective, few were direction selective to a manual moving bar, and even less were direction tuned to motion of a random dot pattern. Both, the proportion of tuning and the anatomical location relative to area MT match descriptions of area V4 and V4t in the macaque cortex (Desimone and Ungerleider, 1986; Logothetis, 1994). It should be noted that the described neuronal properties were predominantly arrived at by manually sweeping a bar and/or RDP of different orientation/direction of motion over the visual field and are thus primarily subjective reports. No main experiment was run on cells not classified to lie within area MT (which can also be seen in the list of recorded cells in appendix B, p. 127).

Anatomical reconstruction of recording sites.

Recording sites were histologically reconstructed after the experiment based on reference coagulation lesions and lesions with electrodes with blunt tips applied immediately prior to perfusion. After initial Ketanest anaesthesia and an overdose of pentobarbital the monkey was perfused intracardially with 5L heparinized, physiological saline (0.9\%) followed by $5 \mathrm{~L}$ phosphate buffer (PB) solution ( $\mathrm{pH} 7.4)$ with $4 \%$ paraformaldehyde. After three months in paraformaldehyde, the brain was stereotactically blocked to contain the full extent of the recording site with four coagulation sites that demarcated the borders of the recording region and including three sites with marker tracks to reconstruct the chamber site. After refixation within $4 \%$ paraformaldehyde $\mathrm{PB}$ solution the brain was cryoprotected with a $20 \%$ sucrose solution and frozen to $-60^{\circ}$ celsius. Brain blocks were cut in $40 \mu \mathrm{m}$ sections at $-20^{\circ}$ celsius with a Reichart-Jung Microtome (2800 Frigocut E) and mounted on gelatine coated slides. We stained sections for Nissl substance with cresyl violet. The positions of recording tracks were reconstructed based on the spatial reference from coagulation sites, stereotactic coordinates, and anatomical landmarks. Figure 2.1 illustrates selected sections along the anterior-posterior (AP) axis (cf. figure legend for details). Our main recording site could be reconstructed based on the reference coagulation lesions and landmark-pin lesions (cf. section 99 in figure 2.1). It was found to correspond well to the region where area MT was expected from the anatomical literature (cf. fig. 2.2, p. 49). It should be noted, that the superior temporal sulcus (STS) of the left hemisphere of the studied brain appears to have a 


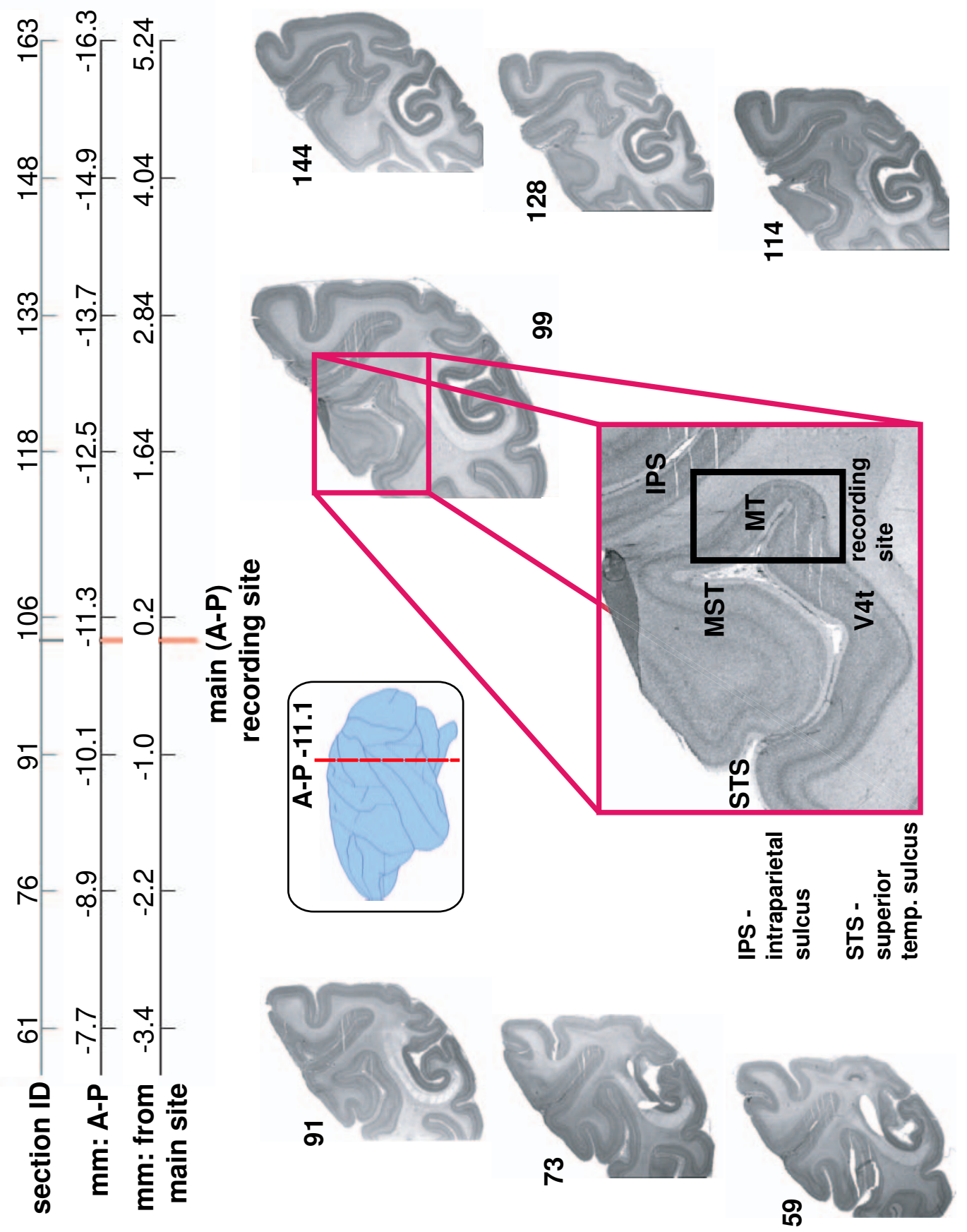

Figure 2.1: Summary of histological reconstruction. Spatial axis (top rows) from anterior (left) to posterior (right) for coronal sections of the brain covers a range of levels that could be targeted by electrodes from within our recording chamber. Our main recording site was found to lie at about $-11 \mathrm{~mm}$ posterior from the APZero level (section number 99), which is shown in the middle axis. The bottom axis plots distance in $\mathrm{mm}$ relative to the identified main recording site to facilitate comparisons between sections. Selected sections allow to track the superior temporal sulcus, which becomes particular evident in sections 91, 99 and 114. Labels in the subsection of section 99 are derived from the literature (Ungerleider and Desimone, 1986; Gattass et al., 1997). The black rectangular region corrresponds to the medialto-lateral and dorsal-to-ventral extent in which all neurons were recorded that went into the analysis of the attentional mapping experiment. 
A

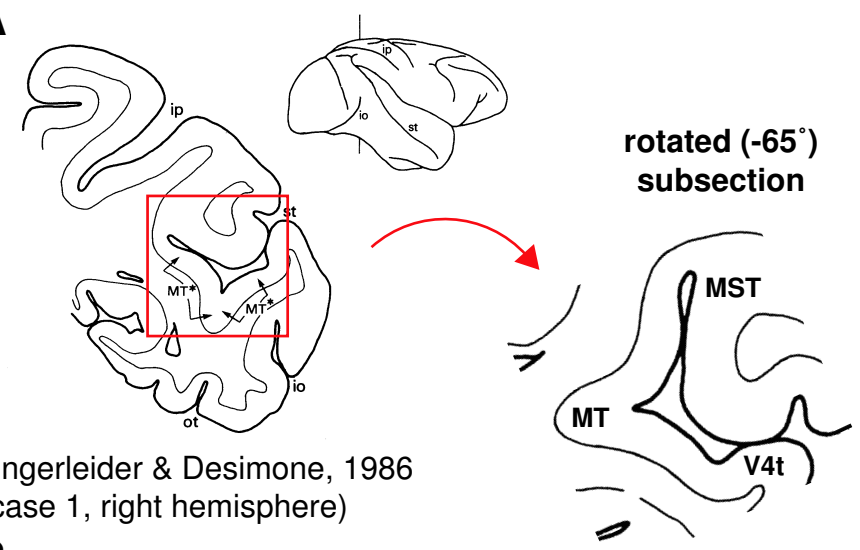

B
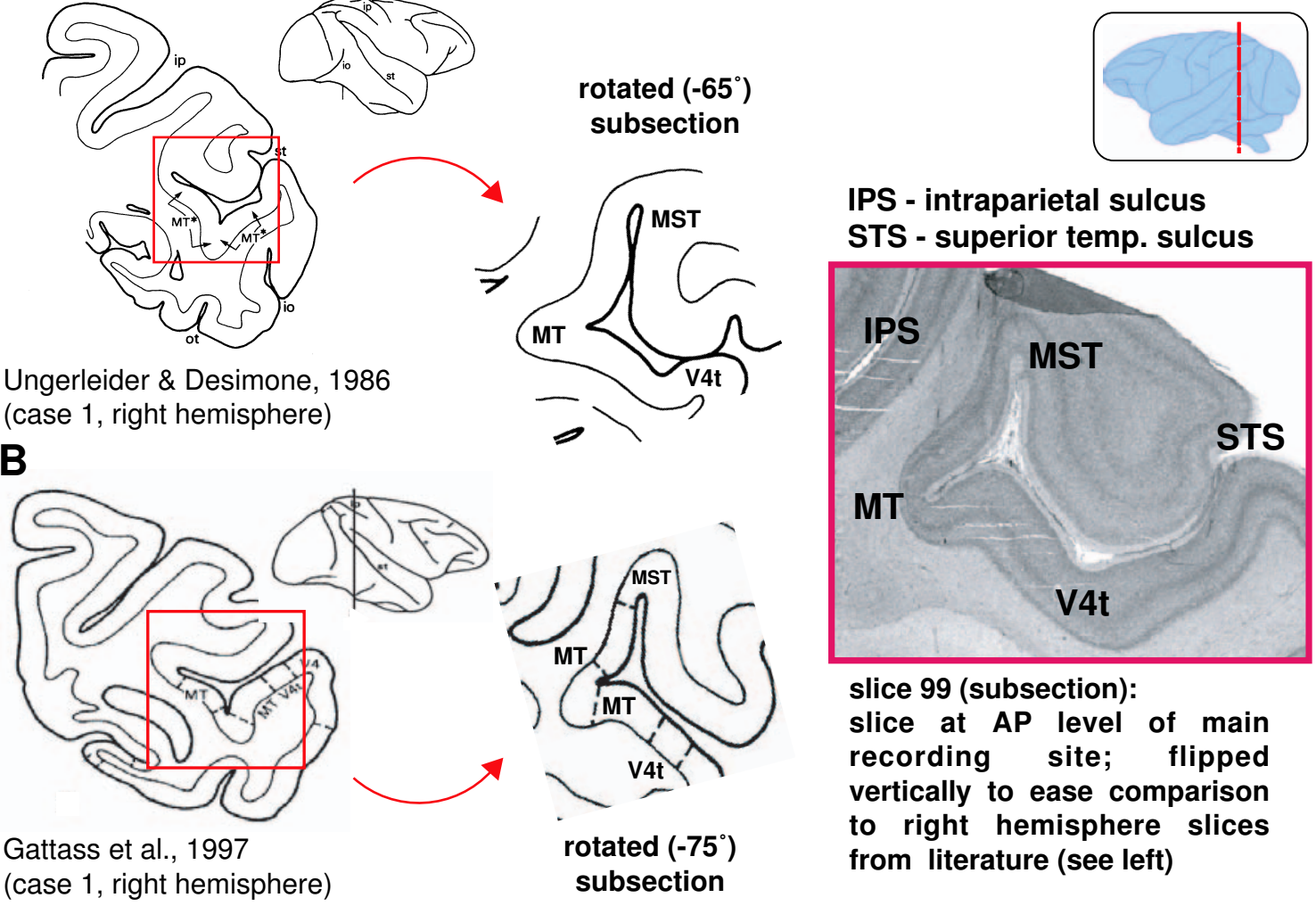

IPS - intraparietal sulcus STS - superior temp. sulcus

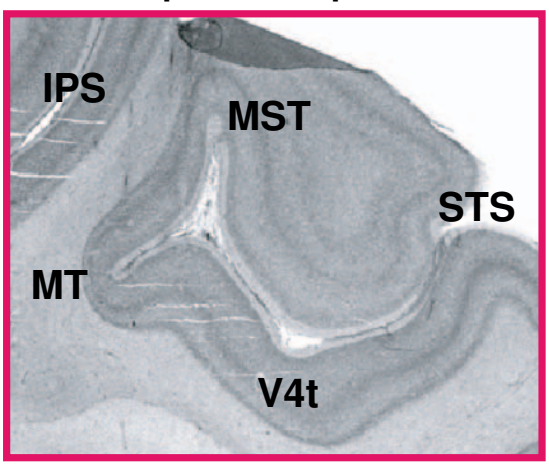

slice 99 (subsection):

slice at AP level of main recording site; flipped vertically to ease comparison to right hemisphere slices from literature (see left)

Figure 2.2: Confirmation of our main recording site with functionally characterized anatomical drawings from two studies concerned with cortical connectivity (and localisation) of area MT (Ungerleider and Desimone, 1986; Gattass et al., 1997). All drawings come from a scientific group which frequently determined the anatomical location of area MT based on a particular strong myelination and a high proportion of neurons selectively tuned to motion direction (Desimone and Ungerleider, 1986). $A$ : Coronal section selected from case one of Ungerleider and Desimone (1986) with the outline of the brain indicating the AP level at which the section had been taken. The selected subsection had to be rotated by $-65^{\circ}$ to fit well to the anatomical form of the superior temporal sulcus in our sections (shown in $C$ ). $B$ : A further example from a more recent study by Gattass et al. 1997. The subsection covering the superior temporal sulcus had to be rotated by $-75^{\circ}$ to match with the superior temporal sulcus of the cresyl violet section in $C$. $C$ : Subsection selected from section 99 at AP -11.2 and flipped vertically to ease comparison with the right hemisphere drawings in $A$ and $B$ (cf. figure 2.1). Comparison of the outline of the superior temporal sulcus of this section with those in $A$ and $B$ shows a close match after rotation of -65 to $-75^{\circ}$ of the cases shown in $A$ and $B$. The need for rotation reflects the finding that the STS in the cresyl violet stained section is far less extensive in the lateral-to-medial axis than would have been expected. This confirms our recording experience before histological sectioning. Labels in $A$ and $B$ are those from the original drawings. Rotated subsections and brain views were slightly modified versions of the original drawings.

peculiar angle and arrangement (cf. fig. 2.2,C) . Compared to anatomical descriptions and drawings of the STS in the literature, the cresyl violet stained STS extends 
from dorsal to ventro-medial in the brain and forms a particular strong medial-todorsal curvature when compared to reports in the literature (cf. fig. 2.2, and for an overview of anatomical drawings of comparable brain sections cf. Ungerleider and Desimone 1986; Boussaoud, Ungerleider, and Desimone 1990; Gattass et al. 1997; Bisley and Pasternak 2000). The consequence of this peculiarity for recording from area MT with electrodes advanced vertically is a restricted lateral-to-medial extent of the region covering area MT, which matches the experience of the experimenter in recovering direction tuned neurons and functionally deleinating area MT.

\subsubsection{Stimulus Presentation \& Behavioral Control}

Visual stimuli were presented with a custom computer program running on an Apple Macintosh G4 Computer. The same software monitored eye position and behavioral responses of the animal, and recorded the behavioral and neuronal data. During the experiment the monkey was seated in a primate chair $57 \mathrm{~cm}$ in front of a monitor with a resolution of 32/33 pixel per degree allowing 960/1280 and 1024/1600 pixel in the vertical and horizontal dimension. The monitor covered a visual field region of about 32 vertical and 39 horizontal degrees in visual angle. Monitors were run with a refresh rate of $75 / 76 \mathrm{~Hz}$, providing a duration of $13.33 / 13.16 \mathrm{msec}$ for each frame of the stimulus. Motion stimuli consisted of random dot patterns (RDPs) presented within a non-moving circular aperture with motion induced by dot displacement within the aperture. Dots moving outside of the aperture were replaced by dots at random positions at the opposite side of the RDP aperture. At the beginning of each experimental session dot positions of the movie frames were generated. Once generated RDPs of the movie frames remained constant throughout the experimental session. RDPs contained 10 dots per square degree of visual angle with indivdual dots extending 2 square pixel. Dots were always white presented on a black monitor background which had a luminance of 0.7 candela during experimental recording. Luminance of the dots was 46.9 candela for all stimuli used in the current project with the exception of the behaviorally relevant target and distractor stimuli of the mapping experiment (see below) which had a luminance of 18.6 candela. These luminance values are averages from fluctuations due to slight variations in background luminance across the duration of the experiment.

\subsection{Experimental Protocol and Conditions}

Experiments began with the isolation of single units based on visual responses to manually controlled light bars or RDPs of adjustable size, orientation and direction of motion while the animal fixated on a white fixation square and performed a change 
detection task of a single moving RDP placed in the visual periphery. Once a visual response was obtained borders of isolated units were mapped manually and fitted with ellipsoids to obtain estimates of RF center position and size. These parameters were used to adjust the position and size of RDPs used to quantify directional and velocity tuning. For this purpose RDPs at twelve different directions and at least three different speeds were presented for more than one second each. Monkeys had to fixate and indicate a detection of a change in the direction of the tuning RDP to obtain juice/tea reward by releasing a lever. Responses to spiral-space motion were recorded while the animal detected a colour change of the fixation square.

We proceeded with the experiment only for well isolated units with a clear Gaussian shaped direction tuning curve. Preferred direction of motion, as well as the initial estimates of RF size and center position were used to adjust the experimental stimulus arrangement as described in the following sections.

\subsubsection{Attentional RF Mapping}

The spatial cueing task deployed in the current study is a variant of the attentional task used previously by Treue and Martinez-Trujillo (1999) (see figure 2.3, A): After obtaining fixation, the monkey had to touch a lever attached to the monkey chair and to encode the location of a stationary RDP presented for 440msec and serving as a spatial cue. After a brief delay (145msec) one target RDP at the cued location as well as two distracters were presented. After a further $160 \mathrm{msec}$ RF probe stimuli were sequentially onset at random locations at the intersections of a virtual grid of 43 to 54 positions covering the RF and its immediate surround. Probe duration was typically $187 \mathrm{msec}$ with an inter-probe blank interval of $27 \mathrm{msec}$.

At random times during a trial targets and distracters changed direction of motion once for $200 \mathrm{~ms}$. The monkey had to detect the direction change of the target RDP while ignoring changes in the distracters. Direction changes could happen from 1200 to $5400 \mathrm{msec}$ during the course of the trial. The extent of the direction change was adjusted to the monkeys performance (depending on size and eccentricity of the target) and ranged from $30 \mathrm{deg}$ to $50 \mathrm{deg}$. The monkey had to release a lever in response to the direction change of the target within a time window of $800 \mathrm{~ms}$. Responses within 200msec after the target change were considered as anticipatory and counted as misses as were lever releases in response to direction changes of the distracters. Targets and distracter stimuli were always adjusted to move in the null direction of the isolated unit as determined by the direction tuning so as to evoke as little a response as possible in order to provide a modulatory range for responses to the RF probe stimulus. To reduce the influence of the attentional target and distracter stimuli further, they were reduced in contrast. Because our primary interest 

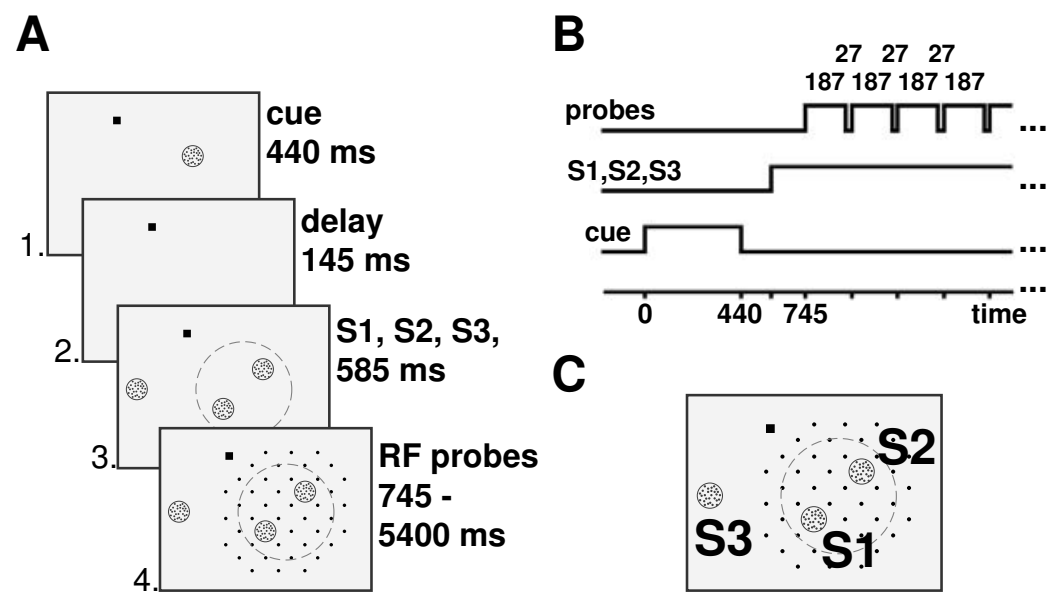

Figure 2.3: Experimental design. A: Each trials began with a stationary RDP cueing the location of the later target stimulus position. When the lever was pressed in response to the cue and a brief delay was passed, the target RDP and two distracters were presented and began to move in the same direction. After a further delay probe RDPs of equal size and moving in the opposite direction of the continuously presented RDPs were sequentially flashed at the intersections of a virtual ellipsoid grid (indicated as grey dots) for $187 \mathrm{~ms}$ each, with an interprobe interval of $27 \mathrm{~ms}$. Tea reward was provided if the monkey indicated the detection of a brief change in the motion direction of the RDP at the target location while ignoring direction changes in the distracting RDPs. $B$ : Time axis of stimulus on and offsets during a trial of the cue (bottom line), target and distractor stimuli (middle line) and RF probes (upper line). $C$ : Typical arrangement and terminology of stimuli for the spatial attention task. On each trial one of the three stimuli were spatially cued to become the target while the remaining two stimuli remained as distracters. S1 and $\mathrm{S} 2$ were always placed within the RF, while S3 were placed in the opposite hemifield at a similar eccentricity as the RF stimuli. Grey dots indicate the position at which the $\mathrm{RF}$ probe stimulus appeared sequentially in random order.

was in the probe responses, the RF probes were always full contrast and adjusted to move in the neurons preferred direction. In order to obtain a baseline response for the RF maps, we always left one time slot of the probe sequence blank.

Spatial arrangement for RF mapping

Position and size of the target and distracters and the grid for the probes were adjusted with a custom program written with matlab (The MathWorks). The main rationale for the adjustment was to ensure (i) that two of the attentional stimuli lie within activating regions of the neuronal RF (ii) that both stimuli in the RF were positioned at equal eccentricity and (iii) that $R F$ probes covered the RF plus its immediate surround. Therefore, the grid was centered at the estimated RF position and extended 1.5 to 2.5 times the RF radius of the longer axis of an ellipsoid RF estimate. In most experiments the grid had an ellipsoid shape with the longer axis running tangentially to the fixation point. Probe radius was always set to half the distance of adjacent grid positions. Target and distracters were always the same size 
as the probes and were assigned to positions of the RF grid with an equal distance to the grid center and always located on the axis perpendicular to the fixation square. This ensured identical eccentricity of the potential attentional targets within the RF. The third stimulus was positioned at a similar eccentric position in the opposite hemifield (cf. figure $2.3 \mathrm{C}$, as an example).

Attentional conditions

During the course of the experiment, conditions were randomly intermixed. In the terminology introduced in figure $2.3 \mathrm{~B}$, the monkey was cued either to attend to S1, S2, or S3. S1 and S2 are located within the RF and conditions with attention allocated to them will thus be called the inside conditions, while attention to S3 represent the attend-outside condition. In addition to the three conditions with peripheral attention there was always a neutral condition indicated to the monkey by a red fixation square and the lack of the stationary spatial cue at the beginning of the trial. It was otherwise identical in visual stimulation during probe presentation. In this fixation with S1S2, i.e. with S1 and S2 within the RF, the monkey had to detect a luminance change of the red fixation square and thus did not require attention to a peripheral stimulus. The control condition was presented randomly interleaved with the peripheral attention conditions during the course of the experimental session.

\section{Sensory RF mapping}

Sensory RF profiles were also obtained in a further condition in which only probe stimuli were presented during the trial. For this sensory condition, (fixation probe condition in the following), the monkey had to detect a luminance change of the fixation square while the receptive field was probed with stimuli at all positions of the grid including those which were assigned to S1 and S2 in the conditions described above. Probes were in all respects identical to those described above. This sensory $\mathrm{RF}$ mapping is similar to mapping regimes reported in the literature and thus allows a comparison with previous studies.

\subsubsection{Direction Tuning: Motion Reverse Correlation}

In addition to the mapping conditions described above we also interleaved trials to monitor and validate the direction tuning of the isolated unit during attentional RF mapping. Direction tuning was based on the motion reverse correlation (MRC) method which is a time efficient way to obtain directional tuning and sensory response latencies of motion selective cells in motion selective cells in macaque area MT and cat striate cortex (Perge et al., 2002; Borghuis et al., 2003). We adapted the MRC method by creating RDPs which changed to one of twelve directions of motion every $80 \mathrm{msec}$ (6 frames), while leaving speed constant. The MRC stimulus was presented in trials requiring the monkey to fixate a red fixation square which 
changed luminance at a random time. It was always positioned in the center of the estimated RF (the central grid position) between $\mathrm{S} 1$ and $\mathrm{S} 2$ with a radius 1.5 times that of S1, S2, and the probes.

\subsection{Data Analysis}

We measured the neuronal responses from successfully initiated trials until behavioral relevant events occured (i.e. color changes of the fixation square, or direction changes of peripherally attended stimuli), or the trial was terminated due to fixation errors or lever releases prior to a change event. Apart from the MRC condition mean firing rates were obtained by averaging spikes of every trial in time intervals adjusted to the respective conditions (see following sections).

\section{Direction tuning}

As noted previously, the attentional mapping experiment was only started when the initial estimate of direction selectivity showed fine Gaussian tuning based on peripherally attended RDPs moving constantly throughout the trial in one of twelve directions until a direction change occured at random times starting earliest $750 \mathrm{msec}$ after RPDP onset. For this classical direction tuning firing rates were averaged across the time intervals from $150 \mathrm{msec}$ after stimulus onset to $750 \mathrm{~ms}$. This allowed to exclude early transient responses and excluded time intervals in which direction changes could occur. Mean firing rates for twelve directions were fitted with circular Gaussians of the form $r M o d e l=$ baseline $+\operatorname{dirGain} * e-0.5(\operatorname{dir} / \text { sigma })^{2}$, where $r$ Model is the predicted response, baseline corresponds to the lower asymptote of the Gaussian, dirGain is the height of the Gaussian, dir the direction of motion relative to the preferred direction, sigma corresponds to the standard deviation of the Gaussian. Gaussian fits were done with custom written software based on a non-linear least squares minimization algorithm (Press et al., 1988), which was given the standard deviation of the response at each direction as the variance measure. Goodness of fit was determined by computing first, the $\chi^{2}$ statistics and the probability that the differences between fit and data could be due to noise, and second, the significance of the correlation of fitted and raw data values. The direction at maximum response of the fitted model was considered the preferred direction and the direction $180^{\circ}$ apart from it was considered the null direction. As a second measure of directional selectivity the directional index (DI) was computed as DI $=1-(r$ Max $-r$ Min $)$ with $\mathrm{rMax} / \mathrm{rMin}$ constituting the response to the preferred and null directions of motion.

\section{Motion reverse correlation}

Directional tuning of the responses to the MRC stimulus was analyzed with a custom program which reverse correlated each spike in a trial with the stimulus value 

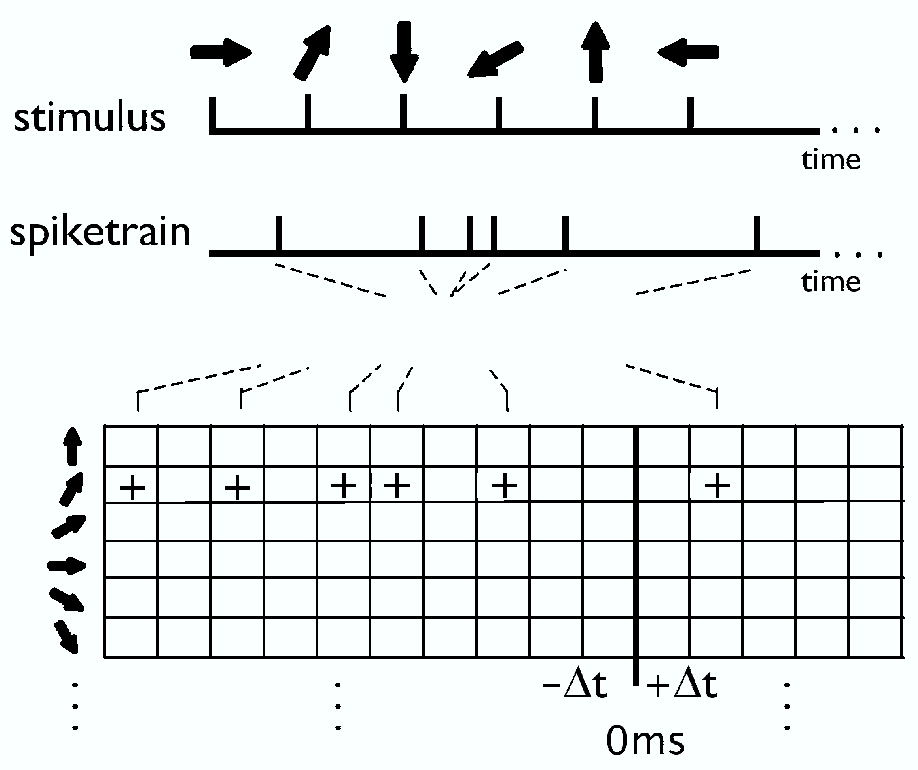

Figure 2.4: Illustration of the reverse correlation method: For each spike of the spiketrain the stimulus value (direction) at $\pm \Delta t$ is found. This is illustrated for a motion direction of $30^{\circ}$ : A spike occurring at $\Delta$ t before the stimulus will be added to the reverse correlogram matrix (bottom figure) at the respective positive time bin, while spikes occurring after the occurrence of the $30^{\circ}$ motion will increment values at the respective negative time bins of the matrix. The resulting matrix is then normalized to reflect the relative probability of motion direction occurrence preceding spike events.

(direction) at $\pm \Delta \mathrm{t}$ with a bin size of 20msec (Eckhorn, Krause, and Nelson, 1993; Mazer et al., 2002; Borghuis et al., 2003; Vajda, 2003). For this purpose the sequence of directions of the MRC stimulus was converted into a continuous stimulus vector (cf. figure 2.4 for an illustration). For each spike during a trial the stimulus values (direction of motion) from $400 \mathrm{msec}$ prior to the spike and up to $200 \mathrm{msec}$ after the spike were added to the corresponding cells of the stimulus-time matrix. The resulting rows (individual directions) were normalized by the number of presentations of the directions, and the whole matrix was normalized by the number of spikes that went into the analysis. The final matrix, i.e. the reverse correlogram, contains values which represent the probability of a motion direction occurrence at time $\Delta t$ given a spike of the neuron at time $t$. The analysis was restricted to stimulus values starting at $200 \mathrm{msec}$ after stimulus onset to reduce artifacts due to luminance onset. Noise level and standard deviation was computed from the uncorrelated part at positive $t$ (i.e. when spikes preceded the stimulus value), because it is physiologically not possible that a stimulus triggers a spike before its onset and thus no correlation is expected to contribute to that part (Mazer et al., 2002). Units were considered for later analysis if values at any time exceeded the noise \pm 2 times its standard 
deviation. The correlogram was sliced at the time bin with maximum response and the directional index was computed as described above. In order to compare the quality of direction tuning of the MRC method and the classical approach circular Gaussians were fit to the slice values according to Gaussian equation presented above, with goodness of fits determined as described above.

Receptive field: position and size parameters

RFs profiles were obtained by averaging spikes in response to the probe stimuli in a time interval of 60-220msec relative to probe onset. Spatial response maps were obtained by interpolating data points at adjacent positions with three to five positions (cubic spline interpolation, matlab, The Mathworks). We always derived RF shape at half maximum response (width at half height) and at two standard deviations above the baseline response to match procedures reported in the literature. Baseline was always the mean response to the virtual probe within the sequence of RF probes, i.e. when there were only S1 and S2 within the RF but no probe. All reported RF analyses were performed after subtracting the baseline response from the RF probe responses. RF center was indexed as peak response position and center-of-mass of the shape as described in the results section.

$\mathrm{RF}$ size was indexed as the square root of the $\mathrm{RF}$ area at half-maximum response (after subtraction of responses from the baseline response, i.e. in the absence of the probe). This RF area was obtained from the interpolated maps and was defined by the vertices at half-maximum response. These vertices form a polygon and allow to apply standard geometric procedures to derive area and centroid parameters ${ }^{1}$ . Our RF mapping involved the presence of three stimuli (S1, S2 and RF probe) simultaneously in the RF which has not been done before in area MT. We therefore encountered not always homogenous elliptical or circular shaped RF shapes at halfheight but sometimes encountered an elliptical or circular central RF shape with one or more small satellite regions with responses above the half-maximum response. These heterogenous RF maps were thus made of more than one polygonal shape. For these cases we added the RF area of each polygonal region in order to derive the RF size. However, we always excluded conditions in which four or more satellite regions were present at half-maximum response.

We applied two major methods to index spatial tuning based on (i) the interpolated two-dimensional RF maps and (ii) on one-dimensional projection of activity along the S1-S2 axis. First, two-dimensional interpolated RF maps provided a position with maximum response, and the RF size measured as the square root of the area within the RF (RF shape at half-height, or two standard deviations above baseline, see above). Second, two-dimensional RF maps were averaged along the

\footnotetext{
${ }^{1}$ The procedures used in this thesis were custom written matlab scripts adapted from information from the following website: http://www.me.psu.edu/sommer/me562/polygeom.doc.
} 


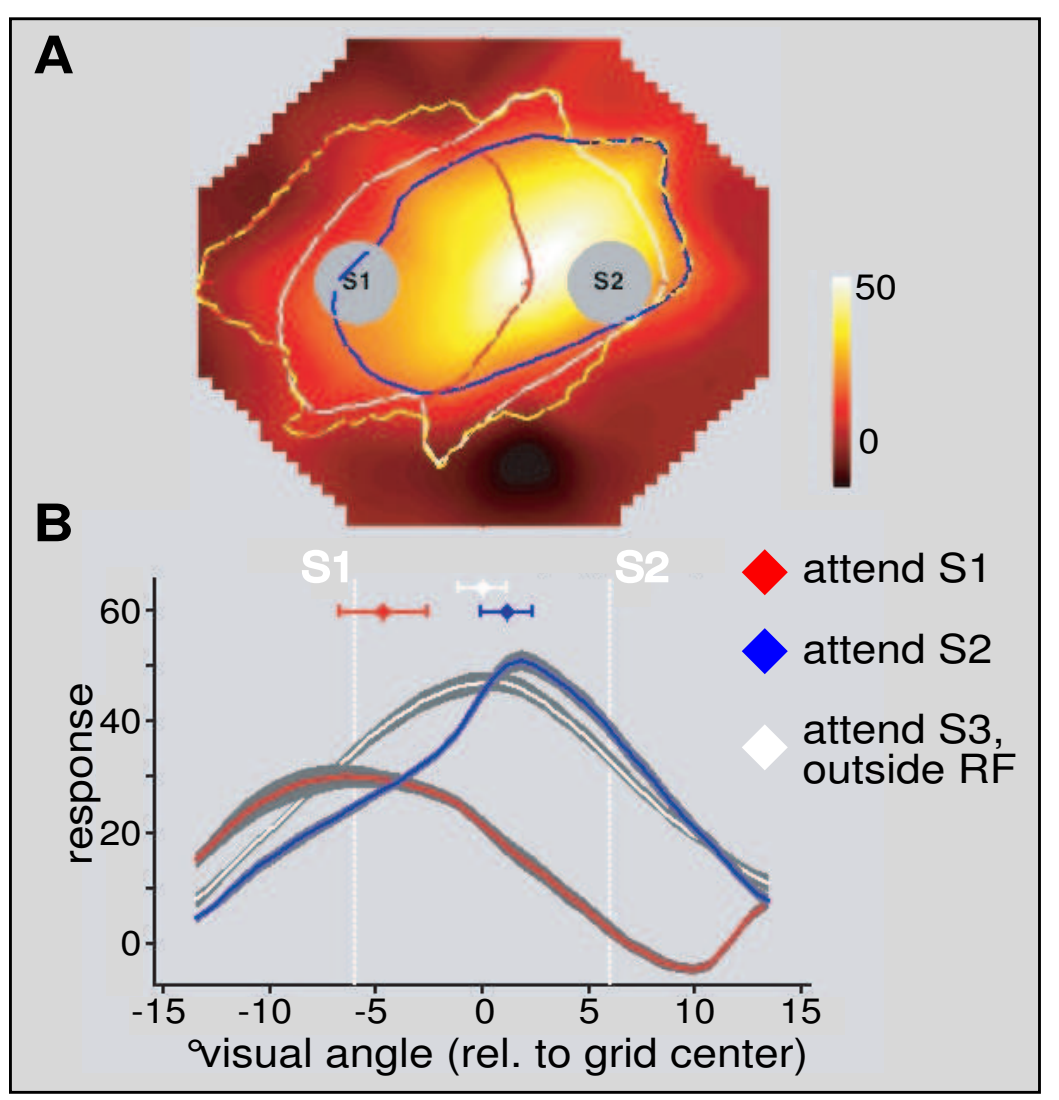

Figure 2.5: Illustration of one-dimensional RF projection. A: Example RF map of a neuron when stimulus 2 (S2) is attended. The RF is rotated to bring stimuli on the horizontal axis. Grey patches indicate the position of the stimuli presented inside the RF (S1 and S2). Coloured outlines indicate the half-height shape of the RFs when attention is directed to S1 (red), S2 (blue), and S3 (outside the RF, white). The yellow outline reflects the area of the visual field which encompasses the RF in any of the attentional conditions. The analysis is based on an one-dimensional projection of activity averaged orthogonal to the S1-S2 axis (and encompassing only regions within the confines of the RF. $B$ : Average activity, i.e. slice, for all three conditions (same color codes in $A$ ) orthogonal to the axis of S1-to-S2 across the yellow outlined area. Diamonds indicate the center of mass (centroid) of the curves and their 0.95 confidence limits which were estimated with a bootstrapping method (cf. p. 58). Vertical white lines indicate the position of S1 (left) and S2 (right) relative to the midline between $\mathrm{S} 1$ and $\mathrm{S} 2$. A spatial shift towards the attended stimulus is evident in centroids that lie closer to the attended stimulus than to the unattended stimulus.

axis orthogonal to the axis made from $\mathrm{S} 1$ and $\mathrm{S} 2$ (the axis from $\mathrm{S} 1$ to $\mathrm{S} 2$ reflects the direction of the shift of attention in the attend inside conditions). The averaging of the RF took into account only those regions of the map that were lying within the $\mathrm{RF}$ in any of the conditions with peripheral attention. The resulting averaged one-dimensional projection of the $\mathrm{RF}$ allowed us to compute the center position of the curve (activity center of mass) and a peak position which were used to compare RFs across conditions (cf. figure 2.5). 
Statistical analysis of one- and two-dimensional RF maps

In order to obtain a measure of variation of RF parameters after interpolation, which was based on the mean response to the RF probes at the positions of the virtual grid, we applied a bootstrapping method. For this test we repeated interpolation of the RF 200 times with responses to individual RF probe presentations drawn randomly from all occurences of the probe at a location in the grid (note that tests with 1000 repetitions did not change the overall result). For each single interpolated $\mathrm{RF}$ we obtained the centroid, the peak response, and the half-height RF size and computed the same parameters after the one-dimensional projection of the RF along the S1-S2 axis. which thus provided a means to estimate the statistical variation of the RF. Differences between RFs (i.e. their centroids, peak positions, or size) were statsitical tested with paired t-tests of the so obtained values.

Attentional modulation index

We computed an attentional index (AI) to index strength of modulation between conditions where attention was directed inside versus outside the RF as follows: $a i=(r 1+r 2 / r 1-r 2)$, AI values can range between \pm 1 with positive values indicating a stronger response for condition one $(r 1)$ than responses for condition two $(r 2)$ and negative values indicating a suppression. 


\section{Chapter 3}

\section{Results}

We recorded from neurons in the posterior bank of the superior temporal sulcus of the left hemisphere of a macaque monkey weigthing $8.2 \mathrm{~kg}$. Enough data for the mapping experiment could be collected for a set of 57 cells which fullfilled all inclusion criteria: (i) They could be held as well isolated single neurons throughout the experiment; (ii) they were recorded from a circumscribed area with highly directiontuned neurons and had receptive field sizes that correspond to those expected for area MT; and (iii) sensory RFs of these neurons were all homogenous and allowed to position the potential target stimuli for the attentional mapping experiments at similarly activating regions within the confines of the RF. For some analysis only a smaller set of neurons could be used as indicated and explained in the respective sections. A detailed list of recorded neurons and experiments conducted with each cell is provided in appendix $\mathrm{B}$.

\subsection{Sensory Properties}

\subsubsection{Directional Tuning}

We obtained for 55 cells directional tuning curves based on reverse correlation of the spike trains to the RDPs which changed directions every $80 \mathrm{msec}$. Based on this method the average direction-selectivity index for these cells was 0.83 ( \pm 0.04 ) (cf. figure 3.1, A). The distribution of direction indices is similar to those previously reported for the population of neurons within area MT (Mikami, Newsome, and Wurtz, 1986; Snowden, Treue, and Andersen, 1992) . For 38 cells (69.1\%) directional tuning could be reliably fit with a Gaussian model (chi square, $\mathrm{p}<0.05$ ). Examples of Gaussian fits are shown in figure 3.2 (dashed red lines in upper panels, see below for details). The average standard deviation (sigma) of the Gaussian fitted tuning was $48.4^{\circ}$ (cf. figure 3.1,B). The isolated neurons were tuned to various preferred directions (cf. inlet in figure $3.1, \mathrm{C}$ ). 

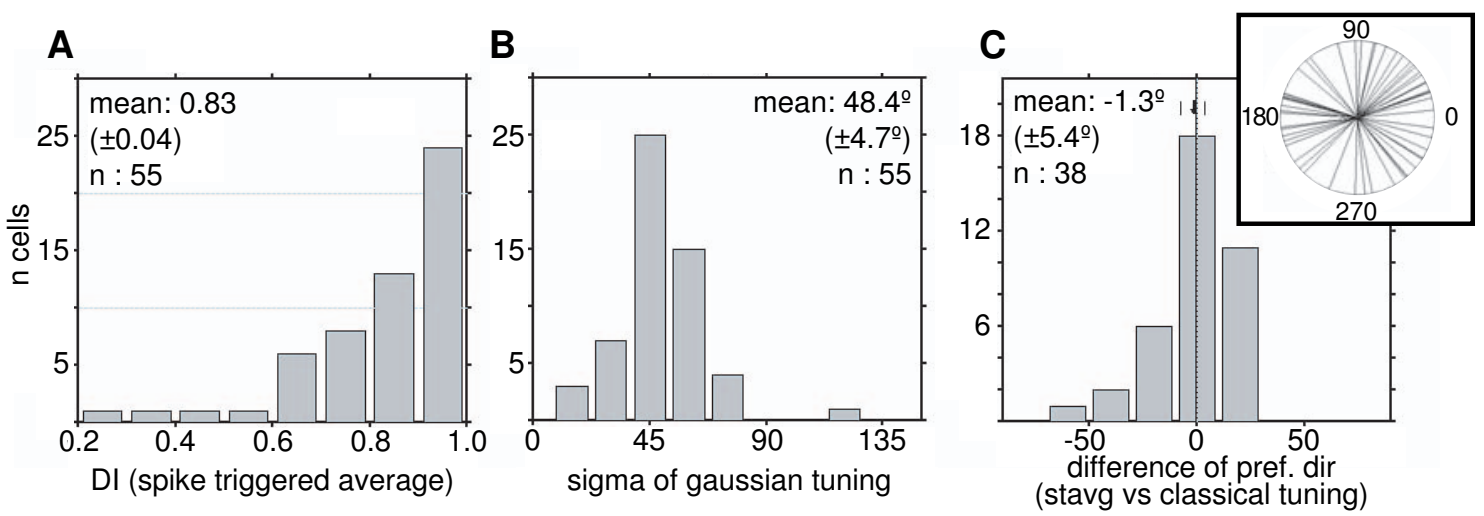

Figure 3.1: A: Distribution of directional indices for cells which could be held long enough for the mapping experiment. DI's are based on the peak and trough of the reverse correlogram obtained with the MRC stimulus. For the 55 cells the mean number of MRC tuning trials was 21.4 (range: 6-55) with each of twelve directions shown on average 11.4 times. $B$ : Distribution of the standard deviation (sigma) of the circular Gaussian model fit to the values of the reverse correlogram at the latency of the peak response. Sigma reflects the degree of selectivity with values ranging near $45^{\circ}$ indicating a fine tuning typical for area MT. $C$ : Deviation of the preferred direction of neurons measured with the MRC stimulus (spike-triggered average, or stavg) and with the classical method with longer duration of uniform motion. The inlet represents the distribution of preferred directions obtained for tuning curves based on all cells tested with the reverse correlation method. It illustrates that there is no sampling bias of neurons within a limited range of direction tuning.

\section{Tuning correspondence of classical and MRC method}

In order to validate the reverse correlation method on our own sample we also obtained tuning curves based on up to twelve directions with a standard method for 39 cells. For these cells we presented RDPs moving in only one direction during each trial and recorded the average firing rates during an 750msec interval (cf. methods section, p. 54). These data were used to estimate the direction tuning of the cells in order to select the preferred and null direction used in the mapping experiment which commenced after this initial tuning test. With this standard method fewer tuning curves were obtained (compared to the MRC method) because we usually recorded these data as long as needed to determine the preferred and null direction of the cell. Therefore, we rarely recorded responses to more than eight directions in more than three trials per direction which were the inclusion criteria for the present analysis (i.e. at least 3 trials for each of at least eight different directions). Despite this small dataset neuronal responses mostly showed direction tuning similar to the direction tuning obtained with the reverse correlation method: The direction index based on this standard method was on average $0.88( \pm 0.04)$ correlating significantly with the DIs obtained with the MRC stimulus $(r=0.67$, $\mathrm{p}<0.001$ ). Similarly, circular Gaussian models (cf. p. 54) fit to both data sets provided almost identical estimates of the preferred direction of the neurons tested 

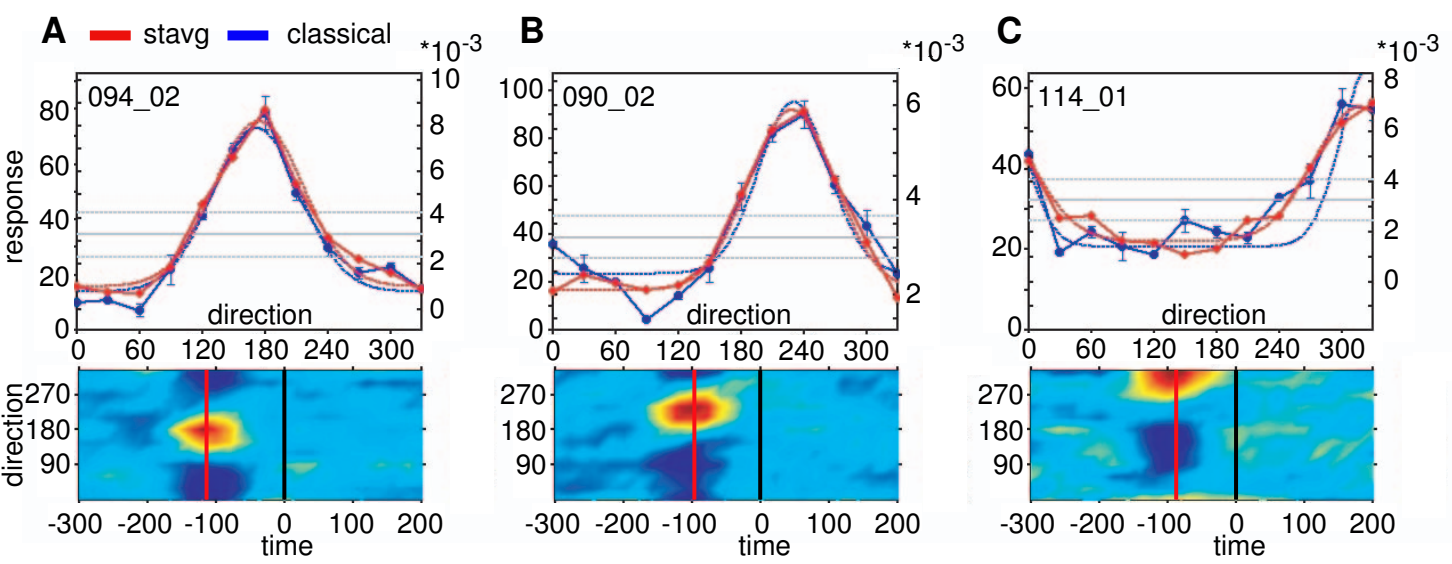

Figure 3.2: Examples of spike-triggered directional tuning. $A-C$ : Tuning curves (upper panels) and flow charts of reverse correlograms (lower panel) for three example cells. The color code of the flow charts reflects the probability that a particular direction (in different rows) is correlated with spike counts at time $\Delta \mathrm{t}$. High/ low values at negative time bins therefore reflect that the respective directions preceded spikes at the given time with a higher/lower probability. Slicing the flow chart correlograms at the peak response across directions (red line) reveals the directional tuning of the neuron. The upper panel illustrates these spike-triggered tuning curves (dotted red line: fitted Gaussian models; straight red line: observed values). Blue lines illustrate the tuning obtained for the same cell with the classical method (average firing rates to directions of motion shown for extended time periods ( $>800 \mathrm{~ms})$ ). The high overlap of the tuning curves obtained with different measures validates the motion reverse correlation method. Grey straight and dashed lines reflects the mean and two times the noise (standard deviation) of the correlogram (computed as average of values in the positive time range), which provide a statistical tool to accept response estimates as statistically significantly different from random counts. The examples illustrate an almost perfect overlap of directional tuning obtained with different methods. The third example $(C)$ illustrates that the method also works well on single neurons with a notable baseline firing rate and a tuning of a lower signal-to-noise ratio (than in the first two cases). Note that in the examples shown, the spike-triggered average tuning was obtained in trials interleaved with the RF mapping experiment, while the classical method was always run prior to the mapping experiment.

with both methods: Figure 3.1, C, plots the distribution of the difference in degrees of the preferred direction derived from the Gaussian fit to the responses of the standard and the reverse-correlation method. Note that only cells were included for the comparison of the Gaussian parameters for which at least three trials could be obtained for at least eight direction. The histogram illustrates strong agreement on the direction tuning of individual cells (no significant difference between conditions, t-test, $\mathrm{p}>0.05)$. This point is further illustrated by the single neuron examples shown in figure 3.2. Analysis of the responses to the MRC stimulus also provided an estimate of the latency of individual neurons: The peak value of the reverse correlograms over the sample of 55 cells occured on average at $-107 \mathrm{~ms}( \pm 5.6 \mathrm{~ms})$ 
indicating that most spikes are elicited $107 \mathrm{~ms}$ after the preferred direction of the neuron was shown. This response latency estimate fits well to the average sensory latency reported for area MT, which is in the range of about 75 to $100 \mathrm{msec}$ for moving stimuli which simultaneously entails a motion-onset and a luminance-onset (87msec for large field moving RDPs, Raiguel et al. (1999); 94msec for moving bars, Raiguel et al. (1989); and about 75msec for flashed light bars, Schmolensky et al. (1998)). In summary, the set of recorded neurons was fine tuned for the direction of motion, measured with different methods and at different time points and a high degree of consistency of results from both methods. The narrow tuning curves with an average Gaussian sigma of $48.4^{\circ}$, together with a sensory peak-response latency of on average $107 \mathrm{~ms}$ reflect properties expected for neurons in area MT.

\subsubsection{Spatial Tuning With and Without Multiple Stimuli}

The main hypotheses of the project concern changes of RFs under different conditions with peripheral attention to stimuli inside and outside the RF. This investigation will rely on measures of RFs when there are multiple stimuli in the RF, by measuring the response to the RF probe presented at different positions (with motion in the preferred direction of motion) and two RDPs moving in the non-preferred motion direction of the neuron (S1 and S2). As described in the introductory section $1.1 .2(\mathrm{p} .8)$ there are few data on RFs with multiple stimuli in the RF and no previous study has mapped RFs of area MT neurons with a RF probe of the preferred direction in the presence of two additional stimuli moving in the non-preferred direction of the neuron. We therefore included two sensory conditions apart from the attention conditions in order to evaluate changes of RFs in the presence of S1 and S2 and to investigate the difference of various measures of RF size (cf. section 1.1.2). In both conditions the monkey had to detect a luminance change of the fixation square and did not require any peripheral attention: A sensory condition in which only the RF probe stimulus was shown and no S1 and S2 (fixation probe condition), and a condition in which the RF probe stimulus was shown and S1 and $\mathrm{S} 2$ were present continuously throughout the trial and which we term fixation with S1S2 condition.

We also measured RFs in a further condition which involved identical visual stimulation of the RF as the fixation with S1S2 condition but required peripheral attention to the RDP located outside the RF (S3). This attend outside condition can therefore be used to evaluate the influence of two further aspects: First, the difficulty level was higher in the attend outside condition compared to the fixation with S1S2 condition, and second, it involved attention to the same direction as S1 and S2 in the RF, i.e. it incorporated a feature-based influence in addition to enhanced task 
difficulty compared to the fixation with S1S2 condition (which involved the detection of a color change of the fixation square).

The next paragraphs will therefore first describe the comparison of RF measures in the fixation probe condition and the fixation with S1S2 condition, and second, commence with descriptions of differences between RFs in the fixation with S1S2 and the attend outside condition.

Sensory RFs without peripheral attention

Analysis of RFs for the main experimental conditions are all based on the RF probe response of the neuron in the presence of the S1 and S2 stimuli. However, current knowledge of RFs is almost exclusively based on experiments with a single stimulus in the RF (cf. section 1.1.2, p. 8), comparable to our fixation probe condition. A comparison of this condition to the fixation with S1S2 could therefore illustrate the influence of two non-preferred motion direction stimuli on the RF parameters. Previous studies on changes in response strength (rather than spatial RF layout) showed that responses to pairs of stimuli moving in opposite direction are a scaled average of the responses that would be obtained when each stimulus would be presented alone (with a scaling factor close to 0.5, cf. discussion in section 1.1.1, p. 7). According to this finding we expected that response strength to the RF probe is scaled uniformly across the RF in the fixation with $51 S 2$ condition with generally lower response strength compared to the fixation probe condition.

We find a maximum average probe response in the fixation with S1S2 condition of 54.8 spikes/sec. (0.95 conf. interval: \pm 7.2$)$ which is significantly less than the observed average maximum probe responses of 61.2 spikes/sec.(0.95 conf. interval: \pm 8.1 ) in the fixation probe condition in which no S1 and S2 were shown (paired t-tests: $\mathrm{p}<0.01$ ). We obtained a baseline response in both conditions by recording the spikes/sec in an interval in which no probe was shown. In the fixation probe condition we obtained on average 15.1 spikes/sec (0.95 conf. interval: \pm 3.7 ) during this baseline period in which no stimulus was shown. In the fixation with S1S2 condition we recorded on average 14.0 spikes/sec (0.95 conf. interval: \pm 4.1$)$ during this baseline period, in which $\mathrm{S} 1$ and $\mathrm{S} 2$ were present. The response in this latter interval represents the response towards two non-preferred motion directions. From previous studies we might have expected that the response to multiple stimuli in the $\mathrm{RF}$ is a scaled average of the response strength to the single stimuli presented alone. However, it is apparent that the average firing rate of 54.8 spikes/sec to the probe in the fixation with $51 S 2$ condition is far higher than the average of the response to the probe presented alone (i.e. $61.2 \mathrm{spikes/sec.} \mathrm{in} \mathrm{the} \mathrm{fixation} \mathrm{probe} \mathrm{condition)}$ and S1/S2 presented alone (14.0 spikes/sec in the baseline of the fixation with S1S2 condition). More specifically, the influence of the preferred probe stimulus clearly outweights the influence of S1 and S2. This finding is likely due to the influence 
A

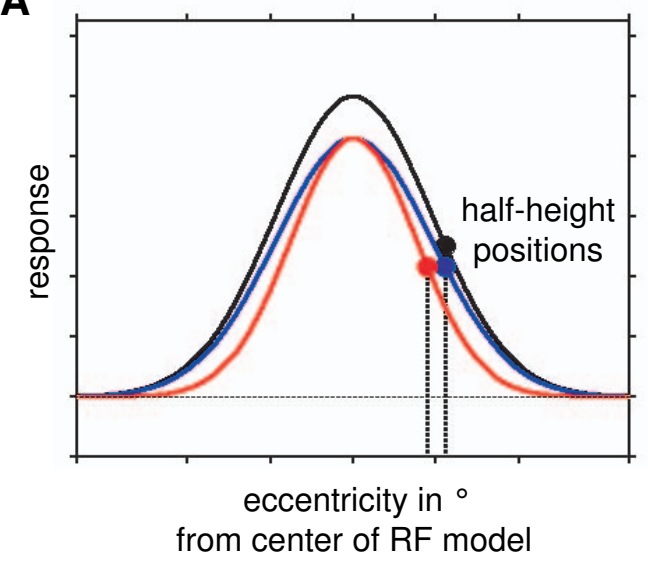

B

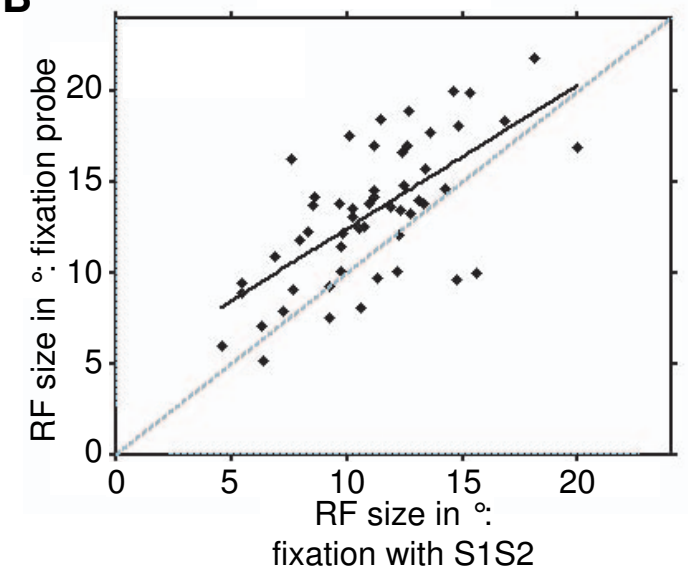

Figure 3.3: The influence of two non-preferred direction stimuli on the sensory RF. A: One dimensional Gaussian models of RFs illustrating theoretically expected and observed RF measures: The black labeled Gaussian could reflect the RF in the fixation probe condition. Compared to this black RF model the blue Gaussian represents a RF which is a multiplicatively scaled version of the black model, i.e. with an identical half-height width, i.e. with identical RF size. The red labeled Gaussian reflects RF parameters that we found in the fixation with S1S2 condition relative to the fixation probe condition: Compared to the black line response strength as well as RF size is reduced. $B$ : RF sizes in the two sensory conditions (attend to luminance change of fixation square) mapped without (y-axis) and with (x-axis) stimuli S1 and S2 in the RF. With the addition of the two stimuli moving in the non-preferred direction of the neurons RFs are on average $19.5 \%$, or $2.2^{\circ}$, smaller.

of the transient onset of the RF probe (which entails a non-selective luminanceonset response) in contrast to the continuous (tonic) presence of S1 and S2 in the RF. The previous analysis showed that there is a significantly lower response to the probe at a single position (which resulted in the maximum average response) in the fixation with S1S2 condition compared to the fixation probe condition. However, it did not show whether responses were lower by the same (multiplicative or additive) scaling factor across all positions of the RF. If RFs in both conditions were scaled by a multiplicative factor then the size at half of the maximum response (the halfheight RF) should be similar in both conditions: Figure 3.3, A, illustrates various 'one' dimensional RF models which are either multiplicatively scaled (blue and black lines), or which differ non-linearly from each other (e.g. black and red lines). In the figure the black labeled RF model could illustrate the RF that we obtain in the fixation probe condition. The red labeled RF model scetches the results which we obtain in the fixation with S1S2 condition: We find the average RF half-height size in the fixation with S1S2 condition to be on average 19.5\% smaller than the sensory RFs obtained in the fixation probe condition (cf. Figure 3.3, B). The difference in RF size was on average $2.2^{\circ}$ from on average 13.3 to $11.1^{\circ}$ which is highly significant (paired $\mathrm{t}$-test, $\mathrm{t}=-5.4, \mathrm{p}<0.001)$. Interestingly, the changes in $\mathrm{RF}$ size are not correlated 
with the changes of the maximal average firing rate to the probes (described above) $(\mathrm{r}=0.07, \mathrm{p}>0.05)$.

We next tested whether the reduced RF size with two non-preferred motion stimuli in the RF is related to the degree of tuning to the direction of motion. Neurons with a stronger direction tuning (i.e. a larger difference in firing to preferred and non-preferred motion direction) might show less reduction in RF size when two non-preferred directions are presented in the RF simultaneously with the preferred direction probe stimulus. However, we did not find any relation between the change of sensory RF size and the direction selectivity index $(r=0.11, \mathrm{p}>0.05)$ or with the tuning half-width $(\mathrm{r}=0.05, \mathrm{p}>0.05)$.

It is important to note that all previous and forthcoming RF parameters (size, centroids, peak positions, response levels) are derived from RFs after subtracting the raw response to the RF probe from the baseline response of the neurons in the absence of the probe. Furthermore, we decided to measure the RF size with the most common method in the literature as the square root of the RF area at half the maximum response (half-height RF) (cf. section 2.3, p. 56). We also evaluated the $\mathrm{RF}$ defined as the region with responses to probes that exceed two standard deviations of the baseline response. In all cases evaluated qualitatively, analysis with this method gave results similar to the half-height defined RF sizes. With regard to the previous analysis, for example, we obtained a significant reduction from $13.3^{\circ}$ to $11.1^{\circ}$ of the half-height $\mathrm{RF}$ size and likewise observed a significant reduction of the $\mathrm{RF}$ size defined by the region with responses above two standard deviation from $21.3^{\circ}$ to $19.4^{\circ}$ (paired t-test, $\mathrm{t}=-2.9, \mathrm{p}<0.01$ ). As already evident in this example, the latter method resulted generally in larger RF estimates which sometimes exceeded the dimensions of the grid of probe positions. This practical problem provides a further reason to use the half-height definition of RFs to arrive at the theoretically relevant $\mathrm{RF}$ parameters.

Spatial tuning: effect of task difficulty and feature-based attention We obtained RF maps with peripheral attention outside the RF to a stimulus in the hemifield opposite to the location of the RF of the neuron. This attend outside condition involved the same visual stimulation in the RF as the fixation with S1S2 condition. However, the peripheral attention task was more demanding for the monkey, which is evident in higher error rates and more fixation errors (see below), and did involve attention towards a motion direction that is also present within the $\mathrm{RF}$ and not preferred by the neuron whose activity we record. Both aspects have been shown to affect neural responses: Increased task difficulty typically enhances the firing rate of visual cortical neurons (Spitzer and Richmond, 1991). Attending to the direction of motion opposite to the preferred direction of an area MT neuron has been shown to reduce the firing rate of the neuron (Martinez-Trujillo and Treue, 


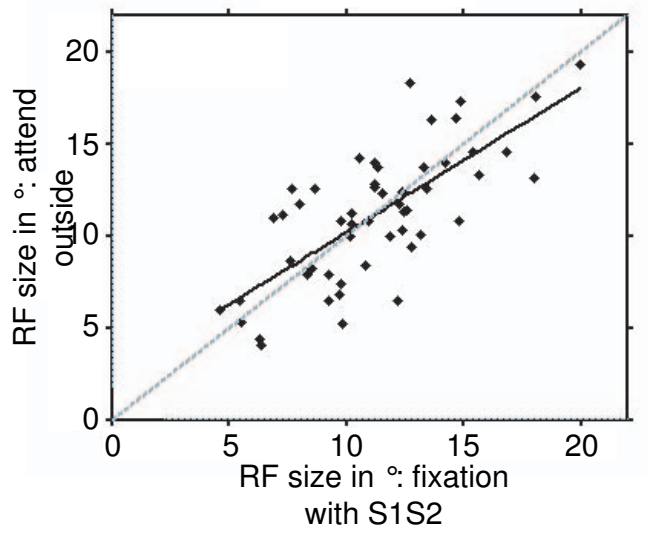

Figure 3.4: RF size (half-height size) in degrees with peripheral attention to a non-preferred motion direction (of stimulus S3, y-axis) and without ( $\mathrm{x}$ axis). There is no systematic difference in RF size despite differences in difficulty and the attentional feature between conditions (non-preferred motion direction in the attend outside condition versus a luminance change of the fixation square in the fixation with $S 1$ and S2 (see text for details).

2004). To test for these effects we compared RF sizes in the attend outside and the fixation with S1S2 condition, and found the RF size to be similar (average reduction of $1.7 \%$ in the attend outside condition, paired t-test, $\mathrm{p}>0.05,0.2$, df 52) (cf. figure 3.4). Furthermore, the average maximum probe response was lower in the attend outside condition compared to the fixation with S1S2 by on average $5.6 \%$ (paired t-test, $\mathrm{p}<0.01(2.7$,df 59). This finding suggests that the feature-based attentional influence outweights the more general and non-selective influences of task difficulty on firing rate. However, the half-height RF size (as well as the standard deviation based size measures, data not shown) was not affected by peripheral attention. Interestingly, changes in RF size were not related to changes in maximal response strength in the different conditions $(\mathrm{r}=-0.14, \mathrm{p}>0.05)$. Our data do not allow to tease unambiguously apart the influence of the covarying aspects of task difficulty and feature-based attention. However, since task difficulty should effect firing rates in a general and non-selective fashion we might argue that its influence is smaller than the feature-based attention effect (also because we corrected RFs with the baseline response which should be effected by enhanced general task demand) and can be neglected. If this is true, then our finding is generally consistent with the feature-based similarity gain model of selective attention (cf. section 1.2.3, p. 16): Attention to a non-preferred direction of a neuron reduces its response gain (i.e. lower firing rate), which should be evident in a multiplicatively scaled response across the spatial RF outline. A multiplicatively scaled RF would have lower firing rates but similar half-height RF sizes (Treue and Martinez-Trujillo, 1999; MartinezTrujillo and Treue, 2004). Both of these predicted effects were found in the attend outside condition relative to the fixation with S1 and S2.

To summarize the previous two analyses, we firstly find differences of the probe response and RF size depending on the presence of two non-preferred motion stimuli (S1 and $\mathrm{S} 2$ ) in the RF: Average maximum probe responses are lower in the presence of these stimuli, but not as low as would be expected from the average of the response 
to the probe alone and to S1/S2 alone. This non-linear scaling of responses is also reflected in a contraction of $\mathrm{RF}$ size when $\mathrm{S} 1 / \mathrm{S} 2$ are present in the $\mathrm{RF}$ compared to the RF size when the probe is presented alone. The second analysis compared the $\mathrm{RF}$ and probe responses in two conditions in which $\mathrm{S} 1$ and $\mathrm{S} 2$ was present in the RF. Peripheral attention to S3, i.e. the RDP moving outside the RF at the same direction as $\mathrm{S} 1$ and $\mathrm{S} 2$, did not affect $\mathrm{RF}$ size, but reduced response strength to the probe. This finding is consistent with a negative scaling factor. 


\subsection{Spatial Attention Effects}

The main hypothesis of this project concerns a change of the spatial position and size of RFs when attention is directed to stimuli at different subregions of the RF and when attention is brought from a region outside the $\mathrm{RF}$ to a region inside the RF. The analysis of attentional effects on spatial sensitivity sets this project apart from most previous attentional studies which were primarily concerned with changes in response amplitude. It is therefore relevant to provide at the outset of the spatial analysis some further background results to validate our approach and to ease comparison with other studies.

\subsubsection{Behavioral Performance}

As a prerequisite for all later analysis, our experimental settings succesfully ensured comparable performance levels in the attentional conditions. Figure 3.5 illustrates that average hit rates did not differ systematically between the attentional conditions. The monkey performed on average $79.3 \%$ correct in the attention tasks. Apart from errors involving early or later lever releases and hits we recorded fixation errors in $16.8 \%$ of all the attention trials. While an ANOVA with condition as factor (including the sensory control conditions) revealed a main effect of condition ( $\mathrm{F}=$ 33.90, $\mathrm{p}<0.01$ ), Tukeys comparison tests showed that attention conditions (attend inside to S1 or S2 and attend outside to S3) did not differ to each other (all p<0.05) but each of them differed to the easier fixation with S1S2 task (all $\mathrm{p}<0.01)$ ).

\subsubsection{Modulation of Response Strength}

Directing spatial attention inside the RF of a neuron typically enhances response (cf. p. 16). However, few studies have investigated the effect of spatial attention inside the RF compared to outside the RF when there are two stimuli in the RF that both move in the non-preferred direction of the neuron (e.g. Treue and MartinezTrujillo 1999; Martinez-Trujillo and Treue 2004). We computed the attentional modulation index (cf. section 2.3, p. 58) between both conditions based on the average firing rate of the neuron in response to the pair of stimuli (S1 and S2) moving in the non-preferred direction in intervals during the trial when there was no probe stimulus presented (i.e. during the sequence of probe presentation but when there was no probe shown). Figure 3.6, $A$, shows the distribution of attentional modulation indices for the set of $n=57$ cells. Positive index values reflect enhanced firing rates in the attend inside compared to the attend outside condition. We found an average $\mathrm{AI}$ of 0.078 , or $16.7 \%$ after conversion from index values to percentage, which is a statistically significant enhancement of responses due to spatial attention 
(one sample t-test, two-tailed, $\mathrm{p}<0.05$ ).

In order to test for the influence of the RF probe stimulus on the strength of attentional modulation we selected the RF probe at the spatial position at which it elicited the maximum average response after subtraction from the response in the same condition but in the absence of the probes (i.e. when only S1 and S2 was present). We then computed the attentional modulation index based on this probe response in the attend-outside (i.e. attend S3) condition and in the attend-inside conditions (attend S1 or S2). We did not find a significant attentional modulation of response strength for this comparison (cf. figure 3.6, $B$ ). The difference of this comparison to the previous analysis (fig. 3.6, $A$ ) is only the presence of the RF probe in the latter analysis. To summarize these results, we find an enhanced response strength with attention inside the RF in the absence of the RF probe. Subtracting this baseline modulation from the response to the compound of S1, S2 and RF probe does not result in any further modulation with spatial attention inside compared to

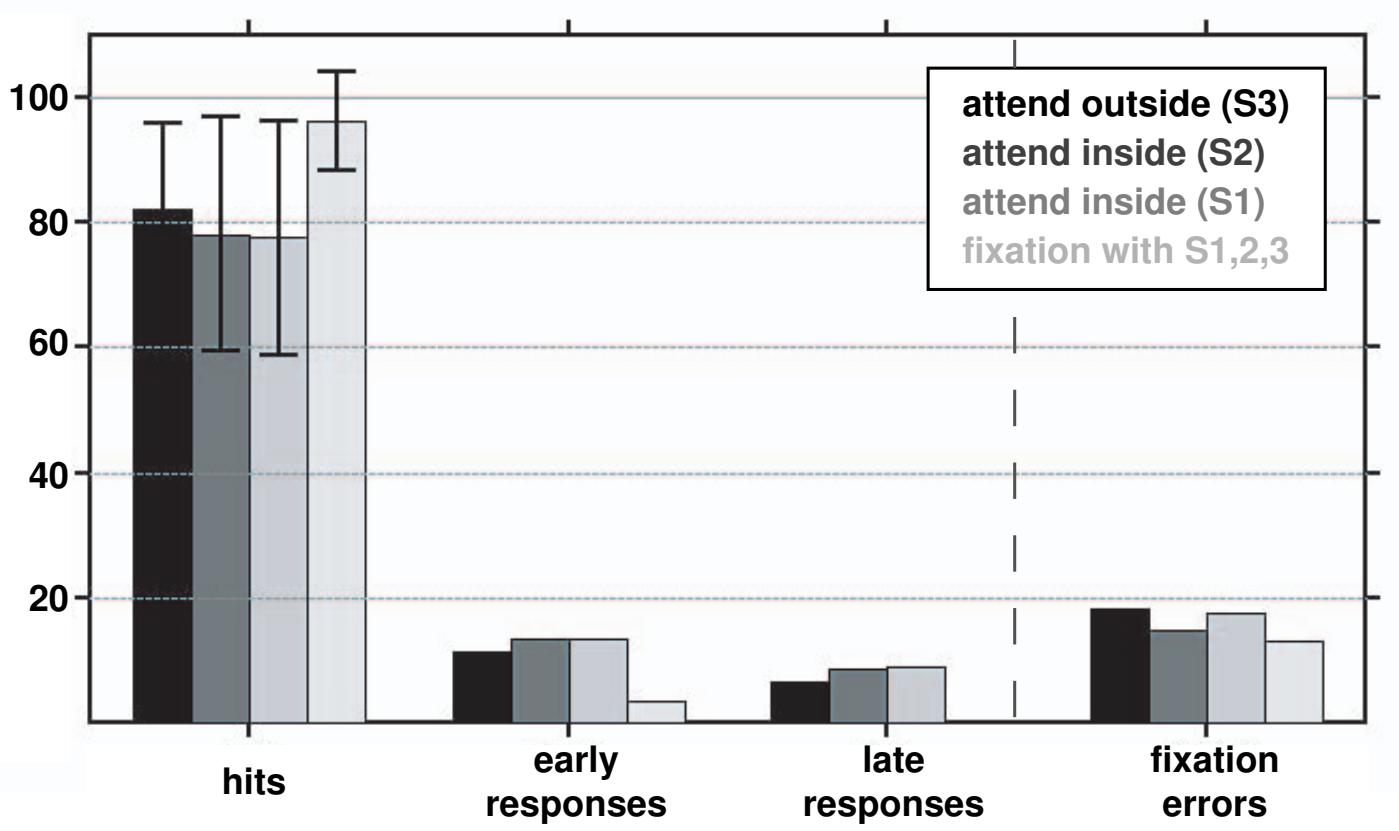

Figure 3.5: Performance rates of the monkey in the attentional tasks (attend S1, S2 and attend outside to S3) and the neutral fixation task with identical stimulation (fixation with S1S2 and S3). Early (late) responses signify error trials in which the monkey responded before (after) the direction change of the target stimulus happened or did not respond at all (included in late responses). Performance levels involving the lever (hits, early and late responses) were computed from all trials during the experimental condition after subtracting the number of fixation errors. Fixation errors included trials when the monkeys gaze left the predefined circular window around the fixation square and are presented as proportion to all trials. The presented average values are based on the performance during 61 experiments during which cells were recorded that went into the RF analysis. 

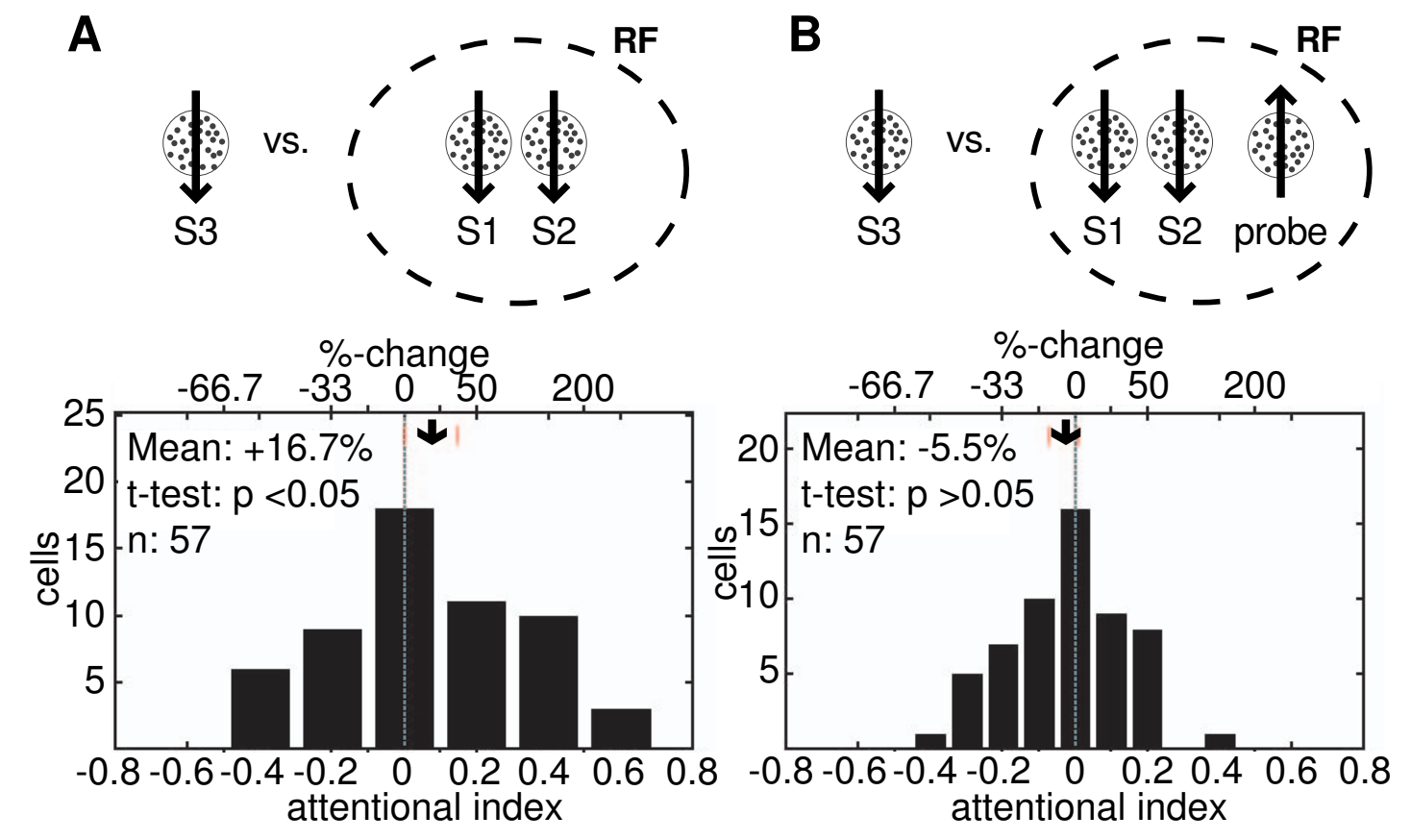

Figure 3.6: Modulation of response strength with attention outside versus inside the RF. A: Attentional influence on responses to the stimuli inside the RF moving in the non-preferred direction in the absence of the probe. We find on average $16.7 \%$ enhanced responses with spatial attention inside compared to outside the RF. $B$ : Attentional influence on the response to three stimuli inside the RF (S1, S2 and the probe stimulus moving in the preferred direction of the cell). There was a non-significant trend for a lower response to the three stimuli in the attend-inside compared to the attend-outside condition. Histograms depict the distribution of attentional indices with the geometric mean provided in the figures reflecting the converted mean index value. Arrows in the graphs show the mean value of the distribution with the small flanking bars representing the pm0.95 confidence limit of the mean.

outside the RF.

To summarize, we find different influences of attention in the presence and absence of the probe stimulus in the RF: Without probe stimulus, spatial attention to $\mathrm{S} 1$ or $\mathrm{S} 2$ enhanced the responses to $\mathrm{S} 1 / \mathrm{S} 2$ by $15.7 \%$ relative to the response to $\mathrm{S} 1 / \mathrm{S} 2$ when $\mathrm{S} 3$ was attended. With probe stimulus present, the response to the probe - after subtraction of the response to S1/S2 - was not further modulated by attention. 


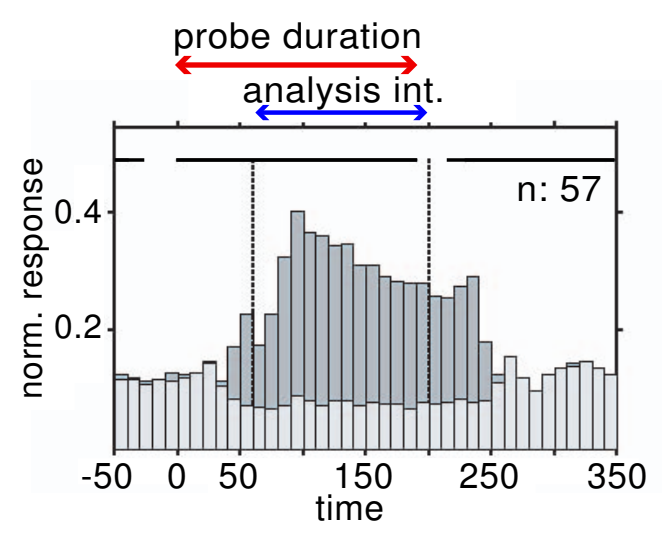

Figure 3.7: Average PSTH to the RF probe stimuli for each of 57 cells which resulted in the maximum average firing rate in the attention conditions (dark grey bars). Light grey bars represent the PSTH during the temporal interval of the attention conditions (i.e. in the presence of $\mathrm{S} 1$ and $\mathrm{S} 2$ ), but in the absence of the RF probe stimulus moving in the preferred direction. Response in the latter condition served as a baseline for the RF analysis.

\subsection{Attentional Effects on Spatial Sensitivity}

The previous analysis provided the background for the analysis of the main hypothetical effect of attention on spatial RF parameters which will be described in the following sections. Comparison of the spatial RF parameters, i.e. the spatial sensitivity, across attentional conditions depends on the interpolation of RF activity profiles based on responses to probe stimuli at different positions (cf. previous section). Across all experiments we were able to place on average the center of twelve RF probe stimuli within the confines of the (half-height defined) RFs in the attention conditions (range of average number of probes per position across experiments: 5-21). Thus, in all cells, probe stimuli could drive the cell response at various positions of the grid. The average peri-stimulus time histogram (PSTH) of the maximum average response to the probes for each cell is shown in figure 3.7. The y-axis reflects the response normalized for each cell in the attention condition according to the maximum average response across all conditions. The average temporal development of activity in response to the probe begins to rise already at $50 \mathrm{~ms}$ from probe onset and remains evident even at $50 \mathrm{~ms}$ after probe offset. We therefore felt safe to use a general analysis interval across all cells of 60 to $200 \mathrm{msec}$ from probe onset (blue arrow in figure 3.7) for obtaining the average firing rates that are used to interpolate RFs. Figure 3.7 also shows the average normalized response of neurons in the attentional condition in the absence of the probe stimulus (i.e. only in the presence of S1 and S2) as light grey bars. Firing rate drops for this time interval when there is no preferred motion stimulus shown at random time slots during the attentional trials. The low response illustrates that the potential target stimuli (S1 and S2) within the RF did not cause a strong response by themselves. This finding illustrates the feasibility of our method. The low response to the potential target stimuli provided a background response that (i) allowed for a range of modulation in response to the probe stimuli, and (ii) established a baseline response for the 
computation of RF maps, which are generally analyzed after subtraction of this background response magnitude.

The following paragraphs will report the effect of attention on the spatial position and size changes of RFs in succession. The results will commence with effects on the center of activity and peak response positions of the average RF slices when attention is directed to either of the inside stimuli (S1 or S2). Shifting attention between different subregions of the RF should provide the maximum shift that can be expected with the current design. Following this analysis, RF parameter changes between the attend-inside versus attend-outside conditions will be presented. This comparison allows to estimate the change in RF size, including a putative RF shrinkage when attention is brought from outside to stimuli inside the RF.

For illustration purposes, figure 3.8, $A$ and $B$ shows the main effect of attention on spatial sensitivity that will be presented in the following in an example cell. As attention is directed to either stimulus $\mathrm{S} 1$ or $\mathrm{S} 2$ within the receptive field, the spatial sensitivity follows this shift. This is most clearly visible in the difference map in figure $3.8, B$. Further examples of difference maps are provided in appendix A, p. 123). The following analysis will provide a variety of specific measures for the spatial diplacement sketched in figure 3.8, $A$.

\subsubsection{Inside-Inside Comparison: RF Shift}

To quantify the effect of attention on the position of the RFs we performed a slice analysis (cf. p. 57, figure 2.5) and analyzed the position of the center of mass, as well as the peak position of the two dimensional RF slices for the population of 57 cells. In order to compare the RF shift across all cells we choose to index the shift with a method that is independent of the eccentricity of the RFs and of the RF size of indivual cells: Figure 3.9, A, illustrates the method: The angle $\Theta r f$ provides a measure which is independent of the eccentricity, because the stimuli inside the $\mathrm{RF}$ (S1, S2) were always placed at equal distance from the fovea (forming always a triangle with the fixation center) which ensured similar task difficulty and thus ease comparison of neuronal responses.

Based on this angle $\Theta r f$ we find the centroid position of the RF slices to be systematically shifted toward the attended target. Figure 3.9 , B reveals that the average $\Theta r f$ of the slice centroid is $14.3^{\circ}$, with positive angles in the histogram reflecting centroids closer to the attended stimulus than to the unattended stimulus. The distribution of $\Theta r f$ values is clearly shifted to the right with its mean being significantly greater than zero (one-sample t-test, $\mathrm{p}<0.001, \mathrm{t}=8.9$ ). Orf will be affected by the separation of the stimuli, i.e. by the maximum separation of the attentional foci when S1 and S2 is attended. Since the positioning of the stimuli S1 
A

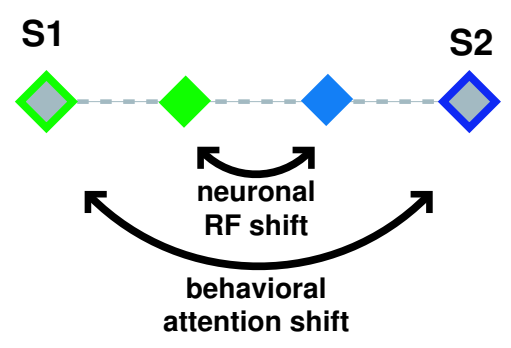

C

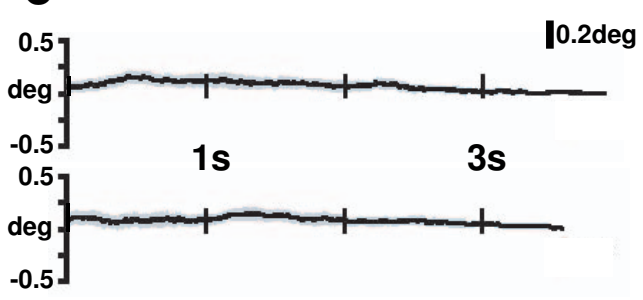

B

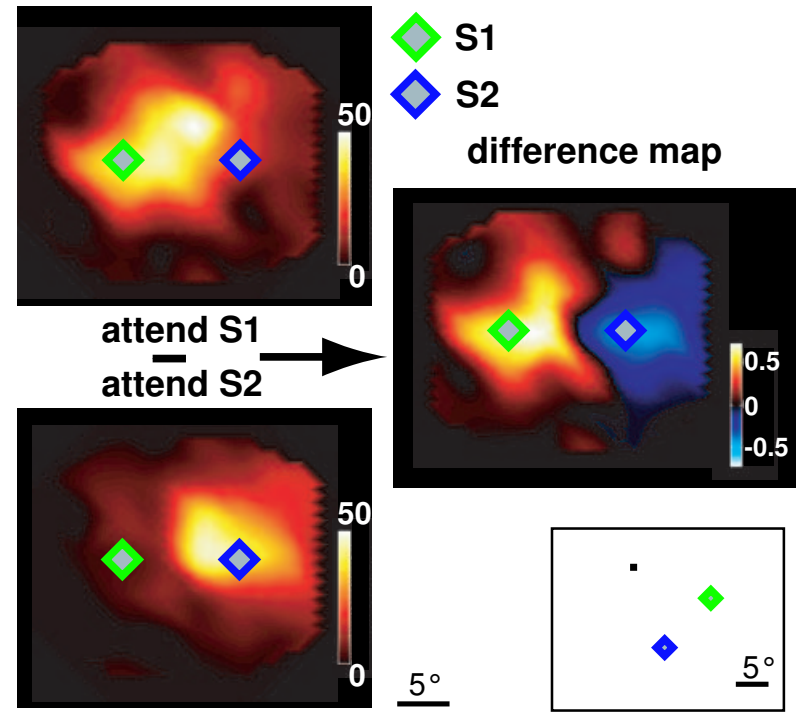

Figure 3.8: Illustration of the spatial shift analysis: attend inside comparison. $A$ : Sketch of the method to quantify shifts in neuronal sensitivity when attention is directed to either of the stimuli inside the RF. The behavioral shift of attention follows the outer diamonds (stimulus positions), while the center of neuronal actitivity (centroid of the RFs, indicated by colored diamonds) is expected to shift on the same axis and in the same direction as the behavioral shift. $B$ : Example of receptive field maps obtained while attention was directed either to the stimulus in the left (top panel, indicated as green bordered diamond), or to the right subregion of the receptive field (bottom panel). Maps are rotated to bring the S1-S2 axis on the horizontal. The neuron illustrates enhanced spatial sensitivity close to the attended stimulus: Responses are stronger to probes close to the attended stimulus while responses were reduced distant to the focused stimulus region. The difference map (right panel) illustrates that spatial sensitivity shifted along the axis of the attended stimuli. The lower panel illustrate the stimulus arrangement including the (black-colored) fixation square on the monitor during the experiment and prior to rotation. $C$ : Illustration of the average spatial deviation of the eye position (fovea) from the fixation point across trials in the condition when S1 was attended (upper panel) and when $\mathrm{S} 2$ was attended (S2). The time axis starts at the beginning of the first probe onset and continues throughout the period in which RF probe responses were obtained. Small grey shading shows the upper and lower 0.95 confidence interval of the eye traces. The traces show that foveal position is very similar in both conditions. The mean distance of the eye position traces between both conditions was $0.116^{\circ}$, which makes it unlikely to contribute to the strong effect on the RF displacement shown in $B$. A further example is presented in figure A.1, p. 124.

and S2 was arranged for each neuron individually the angle at the intersection of the fovea to S1 and S2 (i.e. Os in figure 3.9) will therefore limit the magnitude of the difference of the RF centroids. The absolute spatial shift of $\Theta$ rf by $14.3^{\circ}$ therefore becomes more meaningful if expressed as proportion of the angle $\Theta$ s based on the stimuli positions (i.e. the virtual triangle made by the fovea with S1 and S2). The average proportional spatial shift of the slice centroids is found to be $33.2 \%$ of $\Theta \mathrm{s}$ (cf. 
A

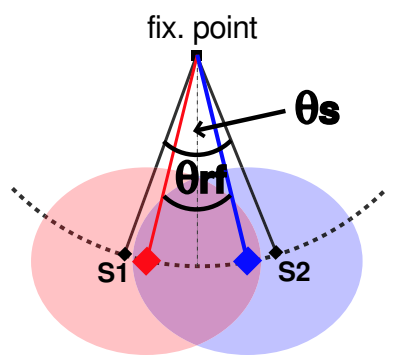

- RF centroid/peak, attend $\mathrm{S} 1$

- RF centroid/peak, attend S2

C

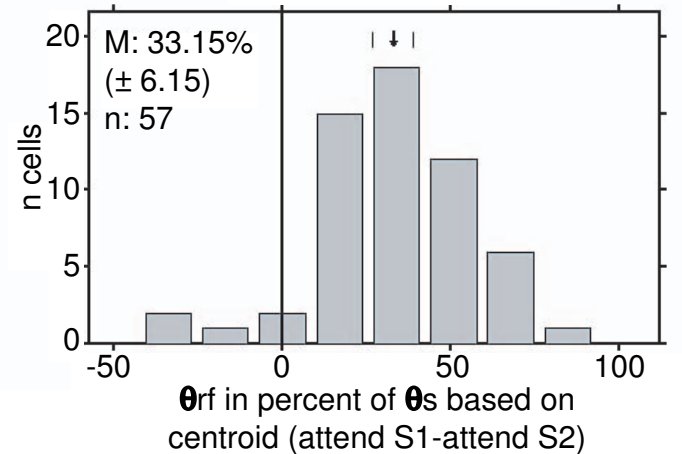

B

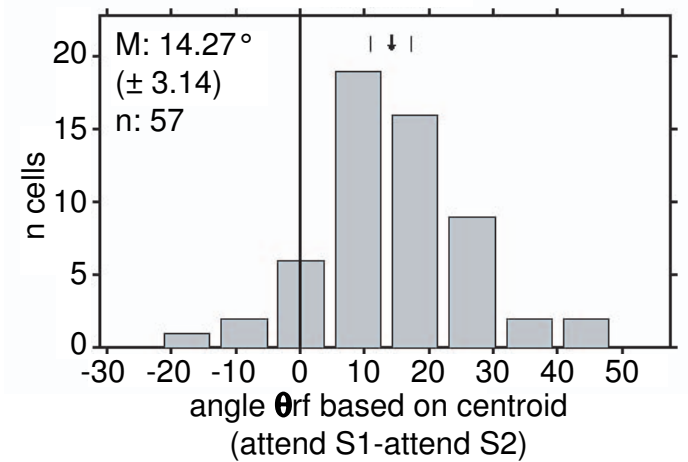

D

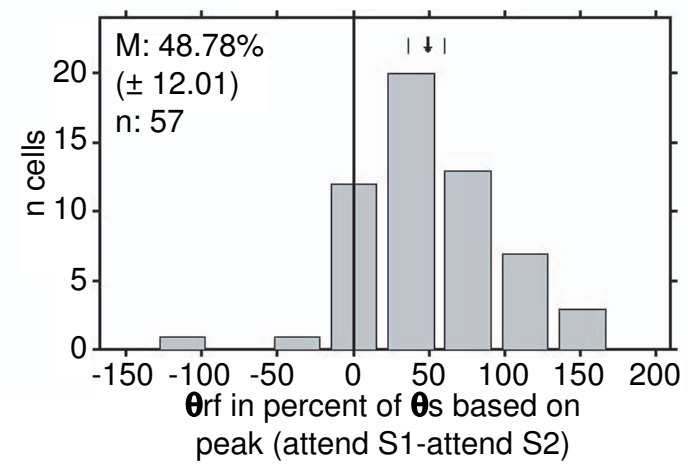

Figure 3.9: Spatial shift of slice centroid and peak when attention is directed inside the RF at S1 and S2. A: Illustration of the angle Orf used for the analysis of the shift of the RF centroids (C, D) and RF peak position (D) between the attend inside conditions. The angle is independent of the eccentricity of the neuronal RFs. The angle $\Theta$ s provides the maximum difference which could be expected to be evident in Orf. $B$ : Distribution of the difference of the RF centroid measured as the difference of $\Theta r f$ and $\Theta$ s when S1 and S2 is attended. Values were arranged so that positive values indicate that the $\mathrm{RF}$ centroid is shifted toward the attended stimulus. $C$ : The angle difference of $B$ expressed as the proportion of $\Theta$ rf relative to $\Theta$ s. Positive values indicate a proportional shift toward the attended stimulus. $D$ : Same as $C$ but based on the slice peak position rather than the centroid. Arrows and flanking lines indicate the mean and the 0.95 confidence interval of the distributions. Text inside the figure denote the mean $(M)$ and the 0.95 confidence interval (in brackets) of the distribution.

figure $3.9 \mathrm{C}$; one-sample t-test, $\mathrm{p}<0.001, \mathrm{t}=10.6)$. In other words, the centroid of the activity profile shifts on average about one third of the angle difference between the stimuli S1 and S2.

The systematic spatial sensitivity shift towards the attended stimulus is not only evident in the RF centroids but is likewise found when considering the RF peak position. Figure 3.9, C shows that the peak response is displaced toward the attended target (indicated by positive $\Theta r f$ based on slice peak) by on average $48.8 \%$ of the angle $\Theta \mathrm{s}$ (which is significantly greater than zero, one-sample t-test, $\mathrm{p}<0.001$, $\mathrm{t}=8.0$ ). Thus, the peak response position of the $\mathrm{RF}$ slice shifts on average even more 
Table 3.1: RF shift results: Attend inside (S1) vs. inside (S2).

\begin{tabular}{|c|c|c|}
\hline & Orf-difference & D-difference \\
\hline centr & $14.3( \pm 3.1)^{* *}$ & $3.5( \pm 0.8) * *$ \\
\hline $\mathrm{f} \Theta \mathrm{s} / \mathrm{Ds})$ & $33.2( \pm 6.2) * *$ & $32.6( \pm 6.2)^{* *}$ \\
\hline peak $\left(\right.$ in $^{o}$ ) & $21.9( \pm 5.4)^{* *}$ & $5.6( \pm 1.5)^{* *}$ \\
\hline 0 of $\Theta \mathrm{s} / \mathrm{Ds})$ & $48.8( \pm 12.0) * *$ & $50.5( \pm 13.4) * *$ \\
\hline
\end{tabular}

than the slice centroids described in the previous paragraph.

As a second measure to index the spatial shift of RFs we computed the euclidean distance of the RF-slice centroids (and peak positions) between the experimental conditions. Similar to the angle analysis presented before the RF centroids are significantly separated from each other when S1 and when S2 is attended. The overall distance of the centroids is $5.6^{\circ}$, which deviates significantly from zero (one-sample t-test, $\mathrm{p}<0.001, \mathrm{t}=-7.6)$. The proportional distance difference between centroid and peak position relative to the distance between the inside stimuli $(\mathrm{S} 1, \mathrm{~S} 2)$ is similar to the proportional angle difference reported above: RF centroids are on average separated by about one third of the S1-S2 distance and the peak positions are separated by about half the stimulus distance. Table 3.3.1 provides an overview of the results reported in this section and show all distributions to be highly significantly different from chance.

The extent of the spatial shift can also be computed as the spatial separation of the RFs in the inside conditions relative to the average size of the RF in the attention conditions. Such proportional shift values provide an additional measure of the RF dynamics and might ease comparison with other studies (with different RF sizes). It should be noted, however, that an $\mathrm{RF}$ shift relative to the $\mathrm{RF}$ size does not take into account the separation of S1 and S2 inside the RF, i.e. the absolute distance of the attentional shift and should thus be viewed with caution. Considering only the distance ( rather than the angle separation) of RFs the centroids differ by $18.2 \%$ $( \pm 4.1)$ of the average RF size of the cells obtained in the attention conditions. This is again highly significant (one-sample t-test, $\mathrm{p}<0.001, \mathrm{t}=-8.6$ ). The respective proportional shift of the peak positions of the RF in the inside conditions is $29 \%$ $( \pm 7.9)$ (one-sample t-test, $\mathrm{p}<0.001, \mathrm{t}=-8.6)$. These values reflect that attention causes a displacement of the RF position by 0.18 (0.29) times the RF size.

To summarize the findings, the analysis of the center of mass and peak position of the one dimensional slice projections of the RFs shows a highly systematic shift of spatial sensitivity towards the attended stimulus position within the RF. This finding from the inside vs inside comparison is reliably evident with all measures tested. 
A

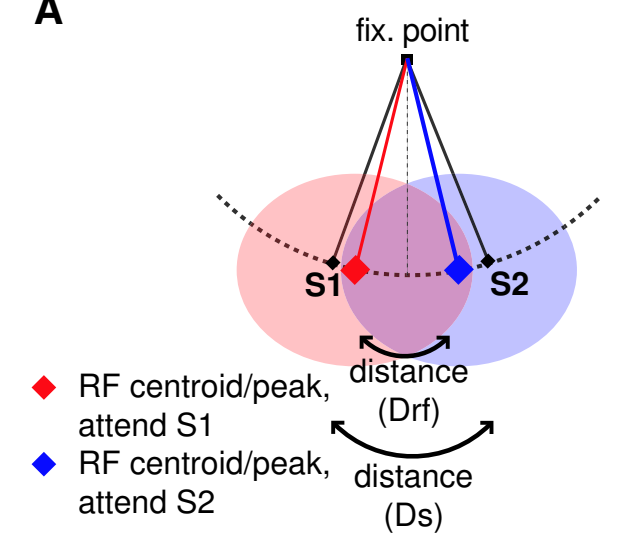

C

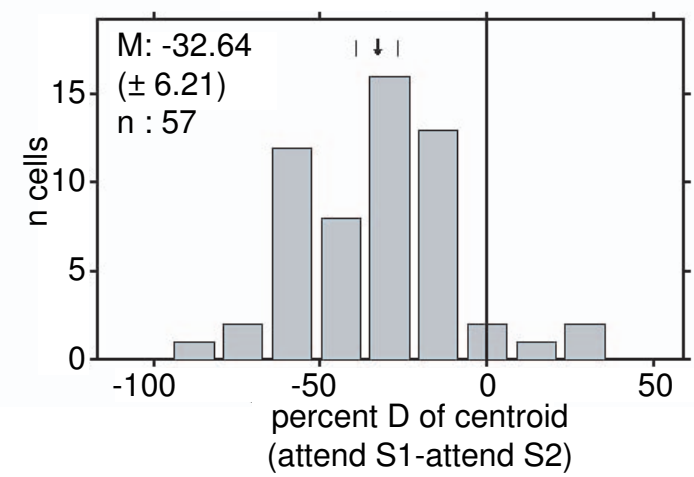

B

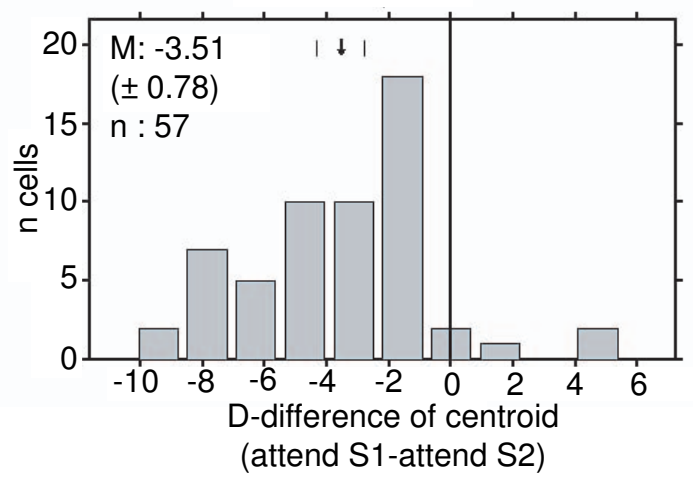

D

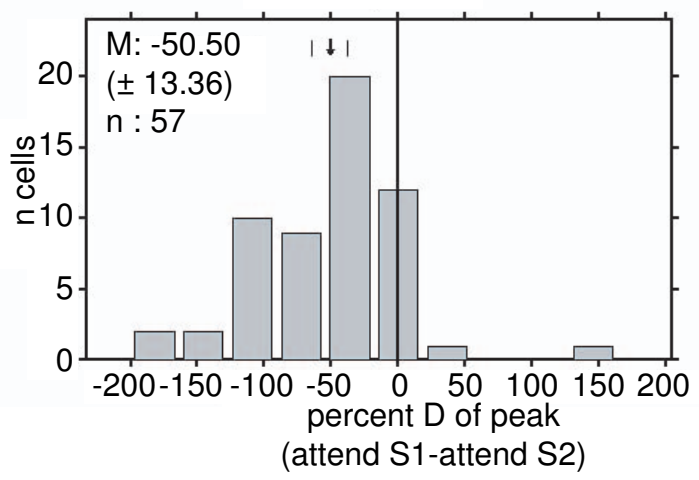

Figure 3.10: Spatial shift of slice centroid and peak when attention is directed inside the RF at $\mathrm{S} 1$ and $\mathrm{S} 2$. A: Illustration of the comparison of the attend-inside conditions similar to figure 3.9 but based on the distances of Drf between the slice centroids/peak positions $B$ : Absolute distance difference of slice centroids when S1 and S2 is attended. Negative values indicate that the RF centroid is shifted towards the attended stimulus. $C$ : The distance difference of $B$ expressed as the proportion of the distance between S1 and S2. Negative values indicate the proportional shift towards the attended stimulus. $D$ : Same as $C$ but based on the slice peak position. Arrows and flanking lines indicate the mean and the 0.95 confidence interval of the distributions.

\subsubsection{Inside-Outside Comparison: Directionality and Mag- nitude of RF Shift}

The previous section revealed a systematic difference of the spatial RF profiles when either of the two stimuli within the RF is attended. This inside-inside comparison provided a measure of the spatial shift irrespective of the RF profile when neither of the stimuli inside the RF is behaviorally relevant. This section reports the analysis when RFs are compared between either of the inside conditions with the condition when S3 (outside the RF in the opposite hemifield) is attended. This analysis is similar to asking whether attention to a position inside the RF acts akin to a spatial attractor of neuronal sensitivity when compared to the RF of the cell when attention is directed outside of it. There are two objectives of these analyses: 
First, an estimation of the extent and directionality of the RF shift when attention is brought inside the RF with a condition of similar task difficulty and demand but with attention to a location non-overlapping the RF. Such an inside-outside comparison is common in studies of spatial attention effects. Furthermore, a shift of sensitivity with attention outside versus inside the receptive field provides a link to a study of RF shifts with attention in area V4 (Connor et al., 1997). This study could only show sensitivity changes with attention to regions outside the $\mathrm{RF}$ (for a detailed discussion of this study cf. introductory section 1.3.1, p. 32). Secondly, this inside-outside comparison controls for potentially spatial specificity and asymmetry of RF plasticity. For example, RF shifts could be particular strong or even restricted to only one of the inside conditions, e.g. a shift only with attention to one of the two stimuli inside the RF which could be due to peculiarities of stimulus placement, or due to a hypothetical, specific 'attentional' tuning of single neurons as suggested in the previous study of Connor et al. 1997. According to this latter suggestion RFs of neurons might have a specific attentional center, which could be independent of the exact position within the RF that is attended. Asymmetric spatial plasticity should become evident in RF comparisons of the outside to the inside conditions.

In the following we will first report results on the basis of an angular and distance based analysis similar to that already used for the inside-inside comparison. In the second part we will introduce a new vectorial analysis in order to answer more specific questions concerning the directionality of the RF shift.

\section{Angular and distance analysis}

In order to compare the spatial shift of RF center locations in the attend inside versus the attend outside condition we first applied an angular and distance analysis similar to that previously introduced in figures 3.9 (p. 74), and 3.10, p. 76). The measures for both analysis are illustrated in figure 3.11, A. With regard to the angular analysis we computed the angle $\Theta$ in ('in' for attend-inside) describing the angular separation of RF centroid/peak and attended stimulus inside the RF (S1 or S2) with regard to the foveal reference position. The same calculation was then done based on the RF centroid/peak location when attention was allocated outside

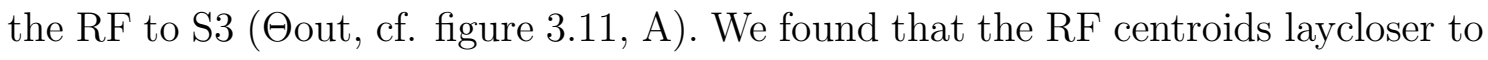
the stimulus inside the RF when that stimulus rather than the outside stimulus was attended: Angle $\Theta$ in was on average $-7.2^{\circ}$ smaller than $\Theta$ out (significantly different from zero: one-sample t-test, $\mathrm{p}<0.001, \mathrm{t}=-7.6$ ). Figure, 3.11, $\mathrm{B}$ depicts the effect as proportional change of $\Theta$ in relative to $\Theta$ out. In other words, we took $\Theta o u t$ as $100 \%$ and report the proportional size of $\Theta$ in relative to it with values smaller than 100 signifiying that RFs are closer to the attended stimulus inside the RF when that stimulus was attended (rather than S3). This analysis reveals that the angle $\Theta$ in was on average only $72.4 \%$ of $\Theta o u t$. 
A

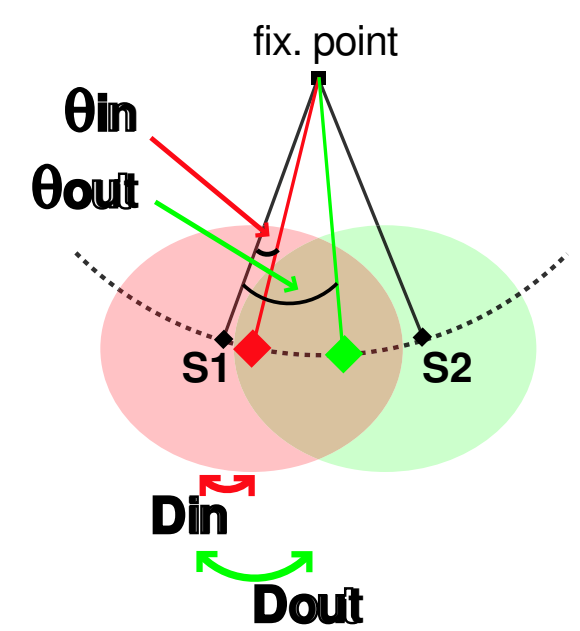

RF centroid/peak, attend $\mathrm{S} 1 /$ attend $\mathrm{S} 2$

RF centroid/peak, attend S3
B

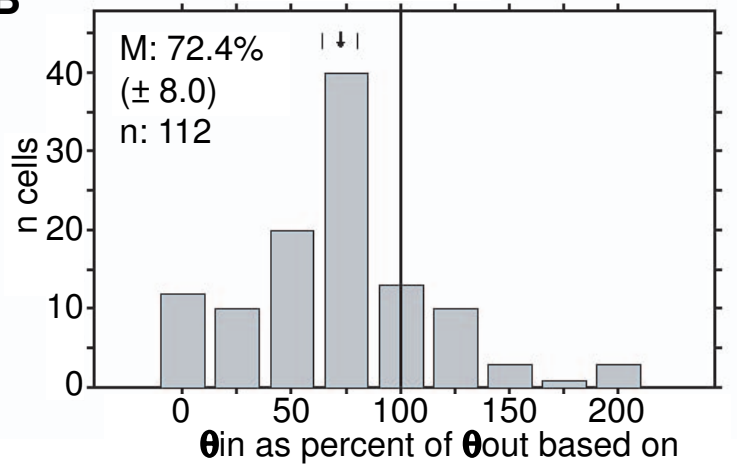

centroid (attend inside-attend outside)

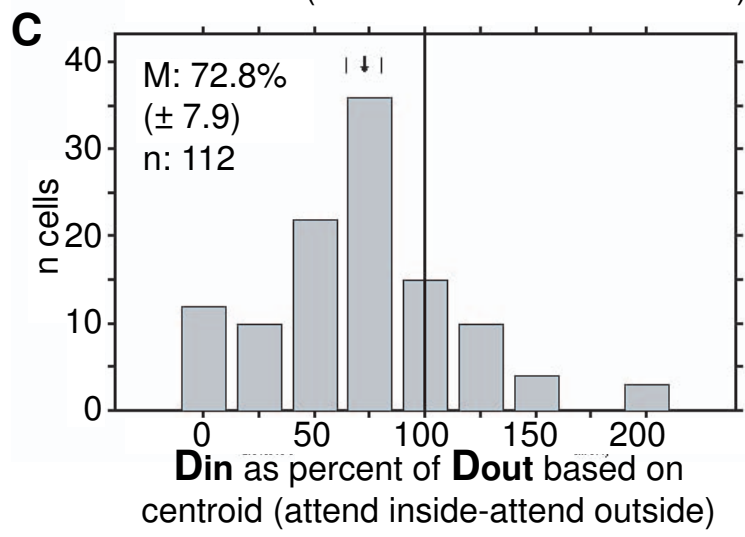

Figure 3.11: Spatial shift of slice centroid and peak: inside-outside comparison. $A$ : Illustration of the comparison of the angle $\Theta$ in and $\Theta o u t$ based on the slice centroid obtained when attention is directed to an inside stimulus (S1 or S2) or outside the RF (S3). B: Difference between $\Theta$ in and $\Theta o u t$ as proportion to the angle made of the inside stimuli with the fovea. Negative values reflect $\Theta$ in that are smaller (centroids closer to the attended stimulus) than Oout.C: Distribution of shift indices, computed for the difference of the RF centroid when attention was directed inside versus outside the RF. $D$ : Same as $C$ but based on the slice peak position. Arrows and flanking lines indicate the mean and 0.95 confidence interval.

In addition to the angular RF shift reported above we also looked at the euclidean distance of the RF centroids in the attend inside versus outside condition. In contrast to the angular measures referenced to the fovea and thus independent of the eccentricity of the cells neuronal RF center, absolute distance will depend on the eccentricity of the cell's RF center, i.e. the same absolute distance of RF centers would provide lower angular differences at higher compared to lower eccentricities). Despite this caveat, our distance-based results are in general similar to those of the angular analysis: RF centroids in the attend inside condition laid closer to S1 compared to RF centroids in the attend outside condition by on average $-1.9^{\circ}$. This smaller distance found in the attend inside condition corresponds to $72.8 \%$ of the distance of the RF center in the attend outside condition (cf. Figure, 3.11, C). Table 3.3.2 (upper two rows) summarizes these results of the inside-outside comparison, 
Table 3.2: RF shift results: Attend inside vs outside

\begin{tabular}{|c|c|c|}
\hline & 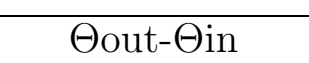 & Dout-Din \\
\hline & $-7.2( \pm 2.1)^{* *}$ & $-1.9( \pm 0.5)^{* *}$ \\
\hline cen & $72.4( \pm 8.0) * *$ & $72.8( \pm 7.9) * *$ \\
\hline peak & $-10.5( \pm 3.2) * *$ & $-2.9( \pm 0.8) * *$ \\
\hline 0 of $\Theta$ out / Dout) & $72.1( \pm 13.4) * *$ & $72.1( \pm 13.0)^{* *}$ \\
\hline
\end{tabular}

which were all based on the RF centroid location (with **marks reflecting highly significant differences to zero, i.e. $\mathrm{p}<0.001)$. The lower two rows provide results for the same analysis but based on the peak position of the RFs. As in the inside-inside comparison of the previous section, the RF shift in the inside-outside comparison is generally stronger when considering the RF peak position. Most importantly, the main effect of an RF shift in the inside-inside comparison is evident with the same measures in the inside-outside comparison. We next go on and analyze the RF shift in more detail with respect to the directional specificity.

Vector analysis and directional specificity

For the following analysis we will consider the peak position of the interpolated two dimensional RF maps rather than the one dimensional projected RF slices used for

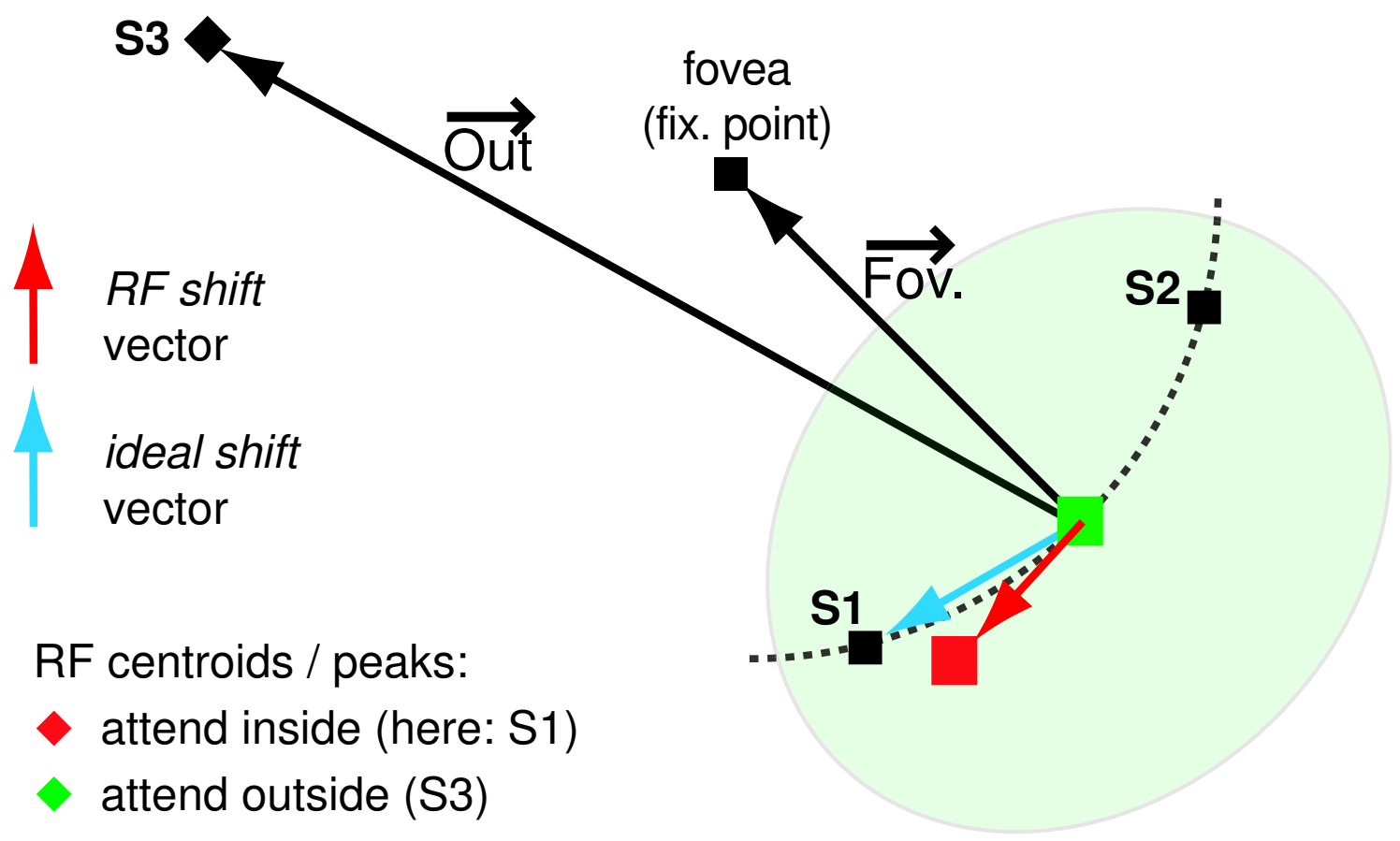

Figure 3.12: Illustration of the arrangement of stimuli (black markers) and RF centers (colored markers) in a typical experiment. If RFs would shift toward the attended stimulus inside the RF (S1 in the figure) from the RF center when attention is directed outside the RF (to S3), then the RF-shift vector (red arrow) should point in the direction of the attended stimulus, which itself can be described by an idealshift vector (blueish arrow). See text for details. 
the previous analysis. The extent and directionality of the spatial shift of the RFs in the inside-outside comparison should be reflected in the amplitude and direction of a $R F$-shift vector which starts at the location of the peak response of the two dimensional RF maps obtained in the outside condition and ending at the RF-peak response of the inside condition. These vectors should on average point towards the attended stimulus inside the RF. They should not be biased towards the location of the unattended stimulus, the fovea, or any other point in the visual field. These vectors are illustrated in figure 3.12 which shows the arrangement of stimuli and observed center of RFs of a typical experiment. According to the terminology of figure 3.12 we would expect that the $R F$-shift vector points in the same direction as the ideal-shift vector, i.e. towards the attended stimulus position within the RF. We therefore computed the angular deviation of the RF-shift vector and the ideal-shift vector. If the $R F$-shift vector contained a component towards the stimulus outside the RF (S3) deviations were arranged to be positive $\left(0^{\circ}-180^{\circ}\right)$, while those $R F$-shift vectors pointing towards the attended stimulus but away from $\mathrm{S} 3$ were assigned a negative sign $\left(-180^{\circ}-0^{\circ}\right)$. Figure $3.13 \mathrm{~A}$, shows that the $R F$-shift vectors clearly point on average towards the attended target stimulus position (S1 or S2) with a median angle of $-6^{\circ}$ indicating a trend of the RF shift to point towards the target and slightly away from the stimulus outside the RF (S3). Circular statistics showed that the distribution was nonuniform and did not differ from the predicted angular deviation of $0^{\circ}$ (Batschelet test, $\mathrm{p}<0.001$ ) (Zar, 1999). The trend to point away from S3 was, however, non-significant. This result shows that RFs shifted towards the attended stimulus inside the RF without a vector component pointing towards the stimulus outside the RF. This is also shown in more detail in Figure $3.13 \mathrm{~B}$ : The distribution is shifted towards smaller angular deviations of the RF-shift and ideal-shift vectors (the figure's y-axis) compared to the angular deviations of the RF-shift vectors and the vector pointing towards the stimulus outside the RF (figure's x-axis).

The previous analysis showed that there is no component of the RF shift that could be explained by the position of S3 outside the RF. However, a previous study reported a tendency of attention-induced RF shifts to be biased towards the fovea in addition to a shift towards the location of attention (Connor et al., 1997). We therefore repeated the previous analysis but aligned RF-shift vectors to the ideal-shift vectors so that positive angular deviations reflect shifts with a component towards the fovea (i.e. the fixation point lied clockwise relative to this angular deviation). The resulting vector distribution is shown in figure 3.14 and illustrates that there was no bias towards the fovea for RF shifts towards the attended stimulus inside the $\mathrm{RF}$, which would be evident in a clockwise skewed vector distribution. The vector distribution is again non-uniform and vector angles are concentrated significantly on the predicted $0^{\circ}$ (Batschelet test, $\mathrm{p}<0.001$ ) with a median angular deviation of - 


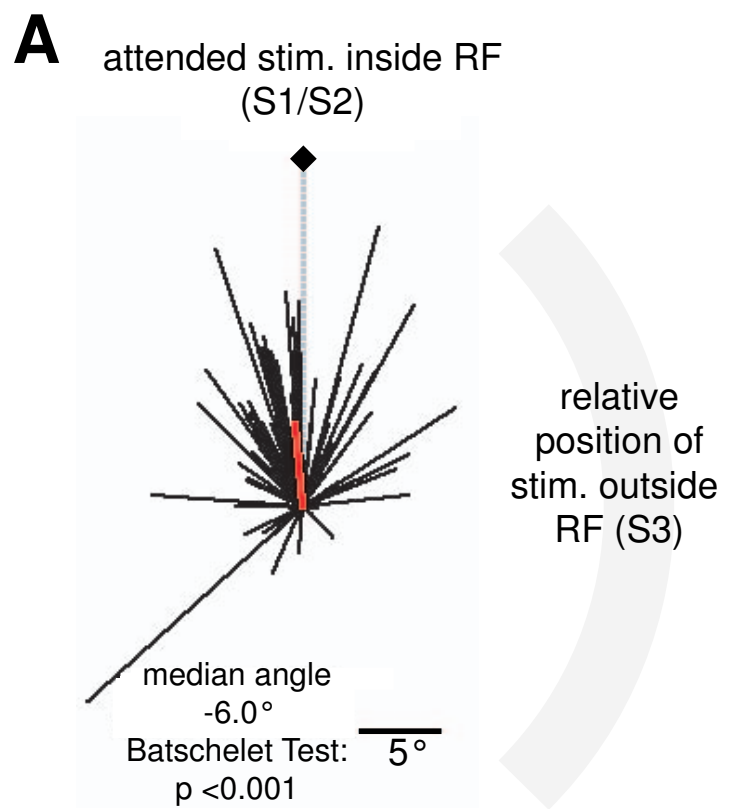

\section{B}

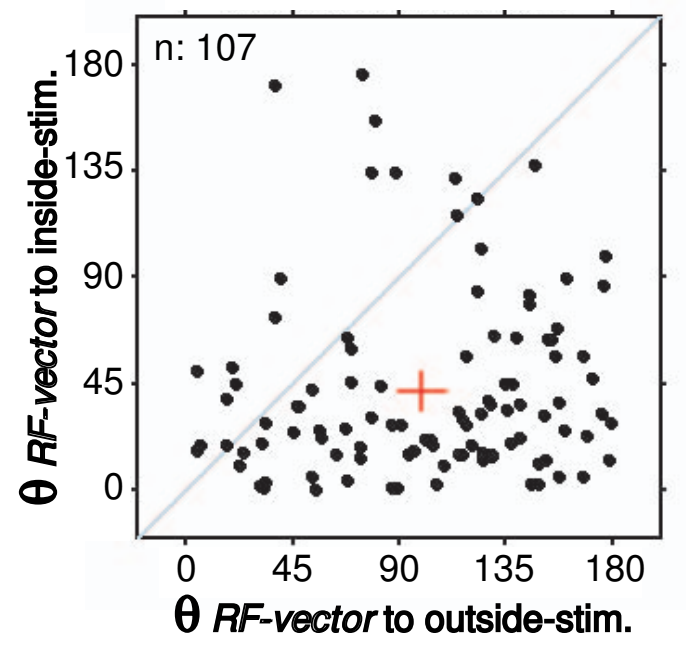

Figure 3.13: Vector distributions of RF-peak responses. A: Distribution of the angular deviation of the $R F$-shift vector from the ideal-shift vector (dashed grey line). Vectors pointing not only to the attended stimulus inside the RF but also in the direction of the stimulus outside the $\mathrm{RF}$ are assigned a positive value and point towards the right from the dashed grey line. B: Angular deviation of the RF-shift vector from the ideal-shift vector (y-axis) plotted against the angular deviation of the $R F$-shift vector and the vector pointing towards the stimulus outside the RF (S3). The graph provides an additional overview of the main result shown in A: $R F$ shift vectors clearly deviate less from the ideal-shift vector compared to deviations to the S3 position.

$6.7^{\circ}$. Thus, attention did not bias spatial sensitivity towards the fovea. In summary, attending inside versus outside the RF act similar to an attractor of spatial sensitivity at the subregion of the attended stimulus inside the RF. This result is evident in shifts of the center of mass and peak response position of the slice analysis and a vector analysis of the peak response positions of the two-dimensional RF maps. The slice analysis was conducted in analogy to the inside-inside comparison of the previous section and shows a high degree of consistency across comparisons. The vector analysis provides further support for the spatial specificity of the RF shift. It showed in addition to the slice analysis that the RF shift is not significantly biased away from the fovea or the stimulus outside the RF. In contrast, there was a slight albeit non-significant trend to point away from these landmarks, which fits well to the idea that attending to the outside stimulus shifted the RF towards that stimulus. This already shifted RF (away from S1/S2 and towards S3) would then shift towards the attended stimulus in the inside condition (towards S1/S2 and away from S3) and would thus deviate away from (rather than towards) the stimulus outside the RF. 


\subsubsection{Spatial Specificity: Shift Asymmetry}

While the result of the previous section shows that the directional shift is reliably found for the inside conditions when tested against the outside condition, RF plasticity could be stronger for one of the inside condition. In other words, attention to one of the stimuli inside the RF (e.g. S1) might attract neuronal sensitivity stronger than attention to the other (e.g. S2) stimulus. In order to test for spatial asymmetry we used the one dimensional RF slices and calculated the significance of the spatial difference of the RF centroid position for each of two inside conditions against the outside condition (based on bootstrapping the RF slices as described in methods section 2.3 , p. 58).

Table 3.3.3 shows the proportion of significantly different RF centroids: in more than half of the neurons (56\%) the RF profile shifted in both inside condition compared to the RF in the outside condition. Almost all other neurons $(42 \%)$ changed their $\mathrm{RF}$ profile significantly only for just one of the inside conditions. The position of the RF centroids of the other inside condition was not significantly different to the $\mathrm{RF}$ position in the attend-outside condition. This finding reflects an asymmetry of
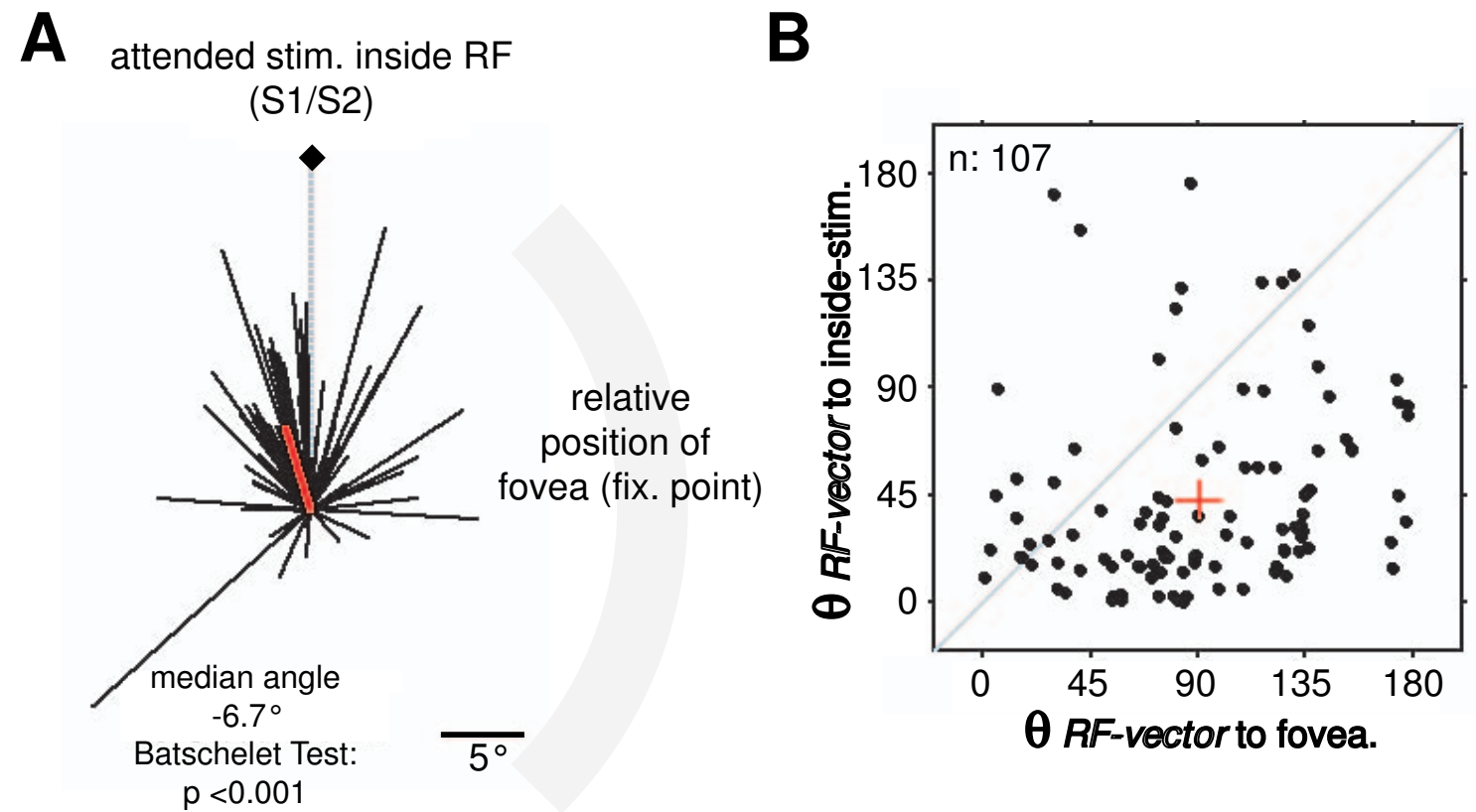

Figure 3.14: Vector distributions of RF-peak responses. A: Distribution of the angular deviation of the $R F$-shift vector from the ideal-shift vector (dashed grey line). Vectors pointing not only to the attended stimulus inside the RF but also in the direction of the fovea (fixation point) are assigned a positive value and point towards the right from the dashed grey line. $B$ : Same results but different illustration as in $A$ with angular deviation of the $R F$-shift vector from the ideal-shift vector (yAxis) plotted against the angular deviation of the $R F$-shift vector and the vector pointing towards fovea. The angle distribution shows that $R F$-shift vectors deviate less from the ideal-shift vector direction compared with the direction to the fovea. 
Table 3.3: RF shift results: Proportions of cells with significant effects (absolute numbe rof cells in brackets).

\begin{tabular}{|l|c|c|c|}
\hline & none & only one $^{*}$ (S1 or S2) & both * (S1 and S2) \\
\hline \% sign. centroid difference & $1.8(1)$ & $42.1(24)$ & $56.1(32)$ \\
\% sign.peak difference & $19.3(11)$ & $47.4(27)$ & $33.2(19)$ \\
\hline
\end{tabular}

the spatial plasticity that could be due to various aspects. The following paragraphs will trace this asymmetry with different analyses.

Shift asymmetry and separation of stimuli to sensory $R F$

One source of spatially specific spatial changes might be a bias of the positioning of the inside stimuli such that one of them were placed further apart from the center of the sensory RF. As a consequence they could have undergone a more extensive shift compared to RFs that are already closer to the center of the RF. To test for this possibility, we obtained the difference of the response magnitudes of probe responses at the $\mathrm{S} 1$ and $\mathrm{S} 2$ position in the sensory, fixation probe condition (where probes were presented at positions occupied by $\mathrm{S} 1$ and $\mathrm{S} 2$ in the attention conditions) and compared it with the extent of the RF shift evident between the attend inside versus attend outside condition. Note again, that RF maps in the fixation probe condition are based only on probe stimuli moving in the preferred direction of the cell which covered all positions of the virtual grid including the locations of the two potential target stimuli inside the RF ( $\mathrm{S} 1$ and $\mathrm{S} 2$ ). The responses to the preferred (and behavioral irrelevant) probes at the target stimulus location, however, did not correlate with the shift of the centroid or peak position obtained when that stimulus was attended in the attention condition $(r=0.06$ and $r=0.03$ for centroid and peak position respectively, both $\mathrm{p}>0.05)$. Thus, a bias, or misplacement, of the positioning of the inside stimuli can not account for the asymmetry of the RF shift.

Shift asymmetry and separation of stimuli to RF in attend-out condition The previous analysis suggests that the spatial specificity of the shift is not systematically related to the peculiarities of stimulus placement with regard to the sensory spatial tuning. However, a different picture might emerge when the shift is correlated with the spatial separation of the stimuli inside the RF (S1 to S2) with the center and peak response of the RF obtained when attention is directed to the stimulus outside the RF (S3). In other words, RFs might shift more towards a stimulus in the inside conditions (attend S1 or S2) when the RF center is further apart from that stimulus in the condition involving attention outside the RF. Figure 3.15, A, illustrates the underlying comparison. The resulting correlation reveals that this assumption holds true: RF shifts are more extensive ( $\Theta$ in is smaller than 
Eout) the larger the separation of the RF centroid in the outside condition to that stimulus (Eout) $(\mathrm{r}=-0.66, \mathrm{p}<0.001)$. A similar relation holds true for the shift and angular separation based on the peak response position of the RF slices $(\mathrm{r}-0.78$, $\mathrm{p}<0.001$; shown in figure 3.15 , B). In summary, this finding could thus account for the asymmetry observed in cells with a significant RF shift for only one inside stimulus condition.

\subsubsection{RF Size Changes and their Relation to RF Shifts}

The previous sections described changes in RF position, i.e. shifts of the center of mass and peak position of RFs with attention. However, the project tried to investigate a second main question apart from RF shift: Does attention also modulate RF size when it is brought from outside the RF (to S3) towards the stimulus inside the RF (to S1 or S2)? In order to investigate this question we computed the $\mathrm{RF}$ size as the square root of the area of the $\mathrm{RF}$ at half-maximum response in the attention conditions. As in all other analyses we subtracted the baseline response in each condition prior to the analysis. Figure $3.16 \mathrm{~A}$, shows the distribution of $\mathrm{RF}$ size changes with attention inside and outside the RF. The RF size variation does not reveal a clear and systematic narrower RF size with attention inside the $\mathrm{RF}$ which would be evident in a regression line with a slope of one and a positive intercept, i.e. a regression line above the diagonal line in figure 3.16 A). However,

A

B

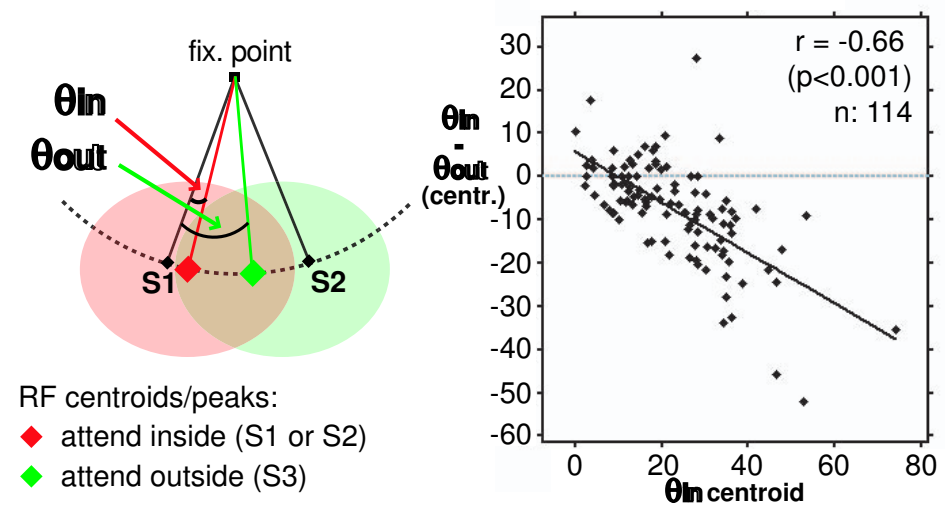

C

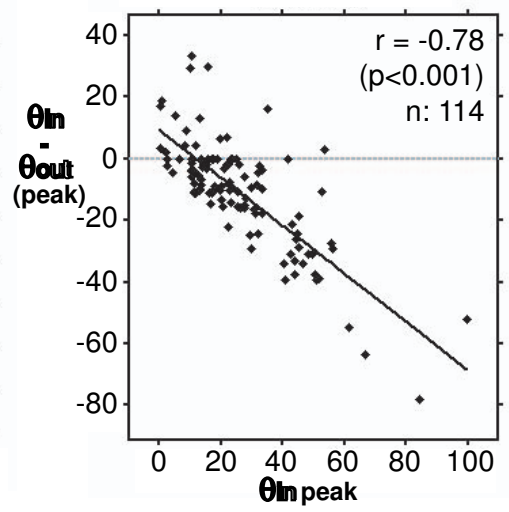

Figure 3.15: Shift magnitude and RF separation from attended stimulus. A: Illustration of the comparison of the angle $\Theta$ in (attend-inside) and Oout (attend-outside based on the slice centroid or peak response position obtained when attention is directed to an inside stimulus (S1 or S2) or outside the RF (S3). B: Difference between $\Theta$ in and $\Theta$ out based on the slice centroid as a function of the magnitude of Eout: Attention to an inside stimulus shift the RF centroid stronger (i.e. $\Theta$ in is closer to the attended stimulus) the more distant the RF in the outside condition (Oout) from that stimulus is. $C$ : Same as B but based on the peak response position of the RF slices. 
A

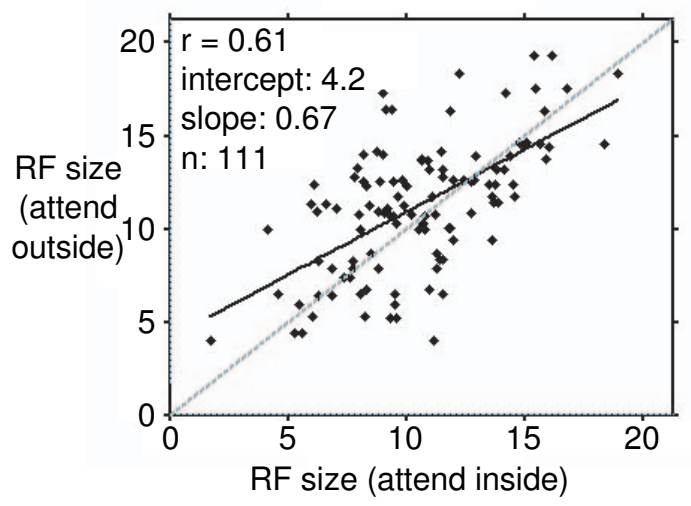

B

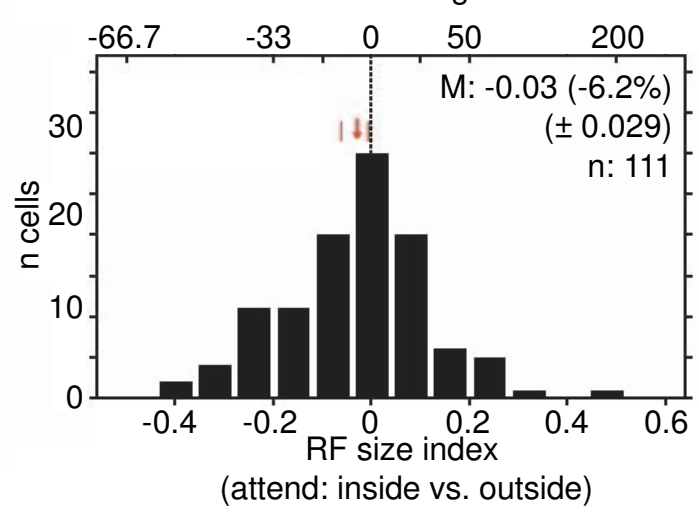

Figure 3.16: RF size changes with attention outside versus inside the RF. A: Distribution of half-height RF sizes with attention oustide (to S3) and inside the RF (to $\mathrm{S} 1$ or S2). The regression line indicates that RFs are not consistently smaller when attention is brought inside the $\mathrm{RF}$, which would be evident in a diagonal regression line above the dotted grey line and a positive intercept. $B$ : Histogram of size indices with values smaller than zero reflecting a smaller $R F$ size in the attend inside compared to the attend outside condition. The distribution is slightly and significantly skewed to the left, i.e. towards narrower RFs in the inside condition (cf. text for details).

quantification of the size differences suggests a small but significant RF shrinkage with attention inside the RF: We analysed relative size changes with a size index $S I=\left(R F_{\text {in }}+R F_{\text {out }} / R F_{\text {in }}-R F_{\text {out }}\right)$, based on the half-height RF size when attention was directed inside $\left(R F_{\text {in }}\right)$ and outside $\left(R F_{\text {out }}\right)$ the $R F$. Size index values can be in the range of \pm 1 with positive values indicating expansion and negative values indicating RF shrinkage in the attend inside compared to the attend outside condition. Figure 3.16, B illustrates that the distribution is slightly skewed to the left illustrating a small but significant suppression of activity when attention is directed to a stimulus inside the RF with an average geometric mean of $6.2 \%$ and a 0.95 confidence limit of \pm 6.0 , which is statistical different from zero (t-test against zero, $\mathrm{p}<0.05, \mathrm{t}=-2.6$ ) (for further analysis concerning RF shrinkage, see below). Note that we have an unequal number of conditions for this comparison due to the exclusion of attentional conditions in which there were more than three satellite regions evident of the RF at half-maximum response (cf. methods section pp. 56). According to one of the hypothesis outlined in the introduction RF shrinkage should be spatially specific around the attended stimulus inside the RF and should thus be related to the RF shift: Stronger shifts of RFs should be accompanied by a stronger RF shrinkage. We therefore investigate (i) the relative systematicity of both effects before we then (ii) provide a correlational analysis of the effects. First, we note that the variability of the $6.2 \%$ average $R F$ size change ( 0.95 confidence limit: \pm 6.0$)$ is more than five times higher than the variability of the RF centroid shift described in 


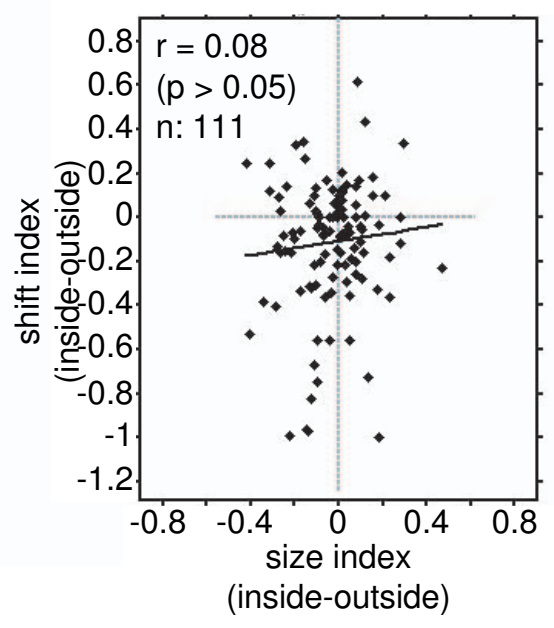

Figure 3.17: Relation of RF size changes and shifts of RF centroids with attention inside versus outside the RF. Negative shift index values reflect a shift towards the attended stimulus, and negative size index values indicate RF shrinkage: There is no systematic relation between shift and size changes (cf. text for details).

the previous sections and shown in the summary of table 3.3.2 (p. 77): We reported that the RF centroid position in the attend inside condition shifted $27.6 \%$ (cf. table 3.3.2: $100 \%-72.4 \%=27.6 \%$ ) towards the inside stimulus relative to the separation of the RF centroid of the attend outside condition and the attended stimulus with a variability of $\pm 8.0 \%$. These measures provide a mean-to-variance ratio of 3.45 which is clearly higher than the ratio of $1.03(6.2 / 6.0)$ for the mean and variance of the RF shrinkage. Even though both effects reach a significance level, the higher variability of RF size changes shows that they are less systematic across neurons.

However, RF shrinkage might nonetheless be systematically related to the RF shift. We therefore computed a shift index for the comparison of the attend inside versus attend outside condition which quantifies the RF shift with the same formula as the size index introduced above (p. 85) based on the RF centroid location rather than RF size. Figure 3.17) shows that size changes (x-axis) are almost equally distributed above and below the horizontal zero line (shifts towards the attended target are reflected in negative shift indices) showing no relation to the $\mathrm{RF}$ shift indices $(\mathrm{r}=0.08, \mathrm{p}>0.05)$. There was neither a correlation of RF size changes with RF shifts when considering the peak position shift rather than the RF centroids as the basis for the shift index $(r=0.07, \mathrm{p}>0.05)$. These results show that $\mathrm{RF}$ shifts towards the attended stimulus are independent of changes in RF size: Shifts are observed for expanding as well as shrinking RFs when attention is brought inside the RF.

In summary, we find a small but significant shrinkage of RFs with attention inside compared to outside the RF. However, the shrinkage was moderate and showed a high degree of variance compared to the RF shift effect. Moreover, RF size changes are not related to the RF shift, i.e. RFs do shrink, but not around attended stimuli as suggested by one of the hypothesis outlined in the introduction. In the following we investigate RF size changes with regard to changes of the response strength, or gain. 


\subsubsection{RF Size Changes and Modulation of Gain}

The main rationale of the following comparison is to investigate the relation of gain and width at half-maximum responses ( $\mathrm{RF}$ size) irrespective of the change of the spatial RF shift. As reported above we find RF shrinkage with attention inside versus outside the RF. We also reported in section 3.2.2 (p. 68) that the response strength (the average maximum probe response) was not significantly different between the inside and outside condition. Taken together, these findings might suggest a shrinkage in the absence of modulation of response gain. However, previous studies involving a single stimulus inside the RF suggest the opposite, namely that response gain changes in the absence of changes in the half-width of tuning functions, which in our case corresponds to the half width of spatial tuning, i.e. RF size. More specifically, previous studies have shown that attention acts on sensory responses consistent with a gain mechanism, which is evident in multiplicatively scaled (Gaussian shaped) tuning curves for motion direction in area MT and orientation tuning curves in area V4 (cf. section 1.2.3 p. 16). This multiplicative attentional influence reflects a change in the gain of neurons in the absence of changes of the ratio of the gain and half-height width, or, in other words, in the absence of a narrowing of the tuning selectivity. We therefore set out to test whether this finding also holds true for changes of gain and size of RFs when attention is directed inside versus outside the RF.

It should be explicitly noted that the analysis relies on the assumption of Gaussian shaped RF profiles which has been suggested to hold true for the majority of area MT neurons (Raiguel et al., 1995). Figure 3.18 A (p. 88) illustrates the hypothetical outcomes based on the noted assumption that the RFs follow a Gaussian shape. According to the multiplicative gain hypothesis we would expect that changes in response strength do not change the half-height width (i.e. the size) of RFs which is illustrated in the left panel of figure $3.18 \mathrm{~A}$. In contrast, the RF shrinkage hypothesis predicts smaller RF-half height sizes either without or with changes in gain (middle and right panel in figure $3.18 \mathrm{~A}$ ). For the analysis we computed the ratio of the maximum response in the attend inside condition and the attend outside condition ${ }^{1}$. From the analysis of response strength presented in section 3.2.2 (cf. figure 3.6, B, p. 70) we already know that there is no significant average change in response strength for this comparison. We then computed the ratio of RF

\footnotetext{
${ }^{1}$ Note that the ratio is a different measure than the attentional index used to measure the modulation of response strength and shown in figure 3.6, B, p. 70. The attentional index reflects a normalization to bring values in the range of \pm 1 , while ratios, on the other hand, can result in arbitrarily large values. In particular, when we compare the average maximum probe response in the inside and outside condition we find an average, non-significant negative index value of -0.03 , corresponding to a geometric mean of $5.5 \%$ lower responses in the attend inside condition. Taking the ratio of the same response values, however, results in an average non-significant positive ratio of 1.01. The distribution of the ratio is plotted in figure $3.16, \mathrm{~B}$.
} 


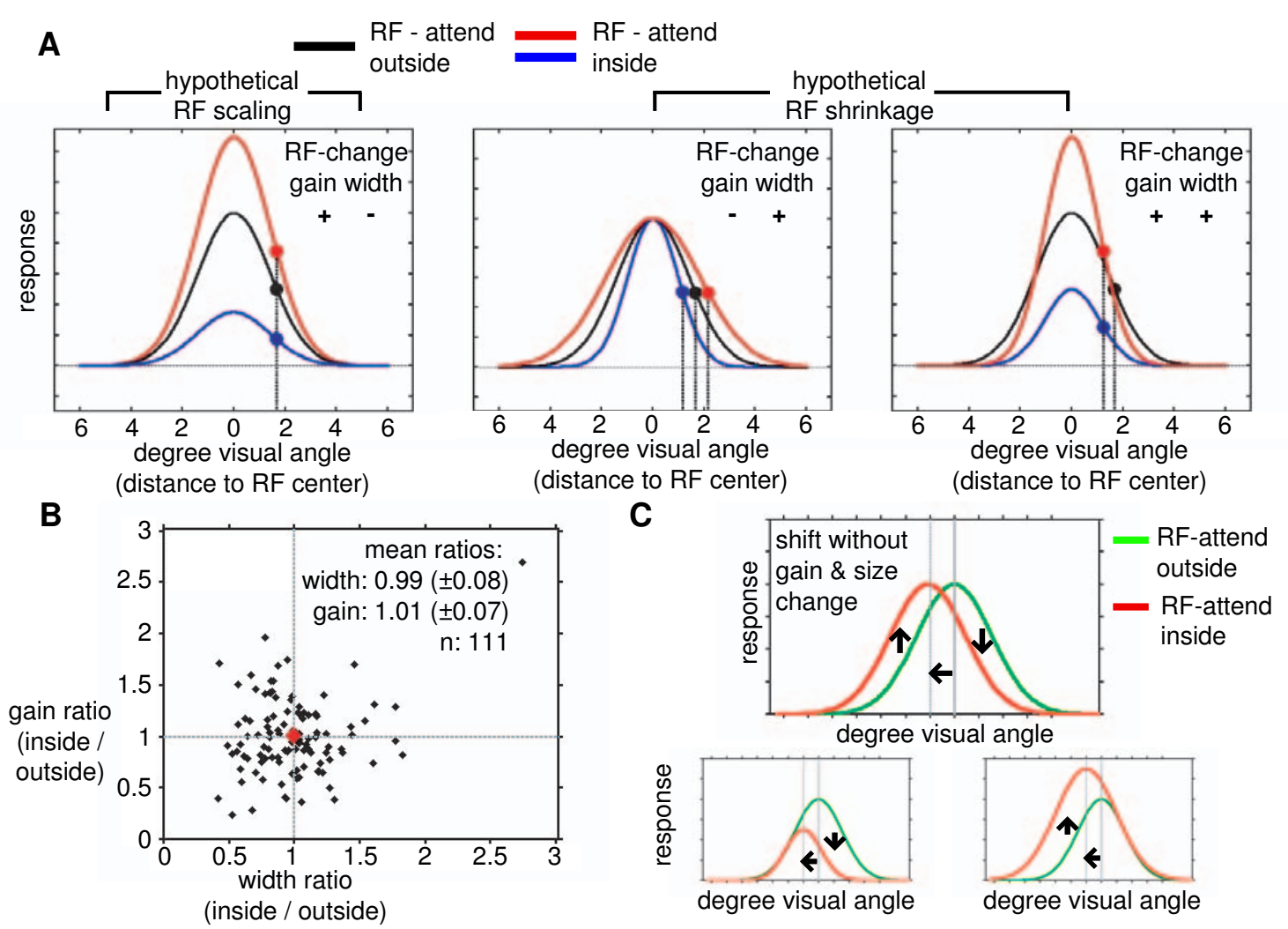

Figure 3.18: RF size changes and its relation to gain. A: Illustration of the relation of gain and width of a one-dimensional Gaussian model (as a simplification of the two dimensional nature of neuronal RF). The spatial tuning curves might change in gain without (left panel) or with (middle panel) a change in size. Otherwise, only the RF size could change without a concomitant gain change (right panel). Obviously RF shrinkage should always involve changes in RF size. $B$ Comparson of gain changes with changes in RF size based on the ratio of the inside versus outside condition for width (smaller values $=$ shrinkage) and the ratio for gain (response strength). There is no systematic relation of changes in gain and width for the population of cells. (cf. text for details, and footnote 1). C Illustration

half-height in the inside and outside condition based on the values presented in the previous section. Figure 3.18 , B shows the result, with a mean ratio of gain and width of 1.01 and 0.99 , respectively. Size and gain ratios are distributed with no systematic relation of changes in gain and RF size (figure $3.18, \mathrm{C}$ ). In other words, this result on its own does neither support any sort of shrinkage hypothesis, nor a pure response-gain hypothesis, because neuronal RF profiles reveal only a marginal change in gain and size.

In summary, in contrast to the absence of clear and systematic gain and size changes the shift of spatial sensitivity remains the strongest significant effect on its own. This finding is illustrated in the shifted (red coloured, ) one-dimensional Gaussian RF model in the upper panel of figure 3.18, C. We find a shift in the absence of clear gain increase or decrease. The lower two panels in figure 3.18, C illustrates 
possible outcomes of RF shifts of the same extent but with concomitant changes in gain. Neither of these possibilities reflect the average effect of our data: We did not find a RF shift that is brought about by a spatially specific gain increase, nor by a gain decrease. Rather, we report a RF shift towards attended stimulus positions without systematic changes in gain and RF size. 


\subsection{Correlation of RF Changes with Performance}

This study reports RF changes as a function of the behavioral state, namely the position of the focus of attention. One underlying source of variations of the RF shift might therefore be the degree of behavioral effort to focus on the attended stimulus. A measure of the effort and ease of focusing the target stimulus in the display is the performance of the monkey in the different attentional conditions. Lower task performance should reflect a stronger demand on the attentional system. In order to test this assumption, we first consider the absolute performance rate (average hit rate in the respective conditions) and correlate it with the extent of the RF shift. Comparing performance and RF shift (as absolute centroid shift of the RF slices) when the monkey attended inside versus outside the RF yielded no significant correlation $(\mathrm{r}=0.10, \mathrm{p}=0.142)$. This shows that absolute performance level was only marginally and non-significantly related to the extent of the RF shift.

In the second analysis we ask whether the difference in performance between two conditions could predict the difference in $\mathrm{RF}$ positions (the degree of the RF shift) in these conditions. Hit rate differences could be a more sensitive measure to reveal a correlation with effort differences because the monkey might favour one of the two conditions (attentional target stimulus positions) due to some peculiarities of a particular experiment ${ }^{2}$. If either the inside or the outside condition would be differentially easy/difficult, the RF shift might be lower/stronger irrespective of the absolute performance level. However, we did not find a systematic correlation of differences in performance and RF shift, i.e. difference in RF centroid position $(\mathrm{r}=0.14, \mathrm{p}=0.068)$. Note that the average performance difference for the inside and outside condition had enough variablity (standard devation: 18.8) to show a correlation if it would be present, despite the only marginal mean performance difference of $2.8 \%$ (only for experiments for which a centroid shift could be computed). The previous correlations only considered the hit rate of the monkey as a measure of difficulty, and thus attentional effort. Hit rates do only take into account the behavioral errors due to responses to non-target events, or misses (early and late lever releases). However, task difficulty is likely also reflected in the amount of fixational errors, i.e. errors during trials that are due to reflexive shifts in gaze. The correlation of the difference in fixation errors between the attend outside and attend inside conditions is, however, not signifcantly correlated with the degree of the RF shift $(\mathrm{r}=0.09, \mathrm{p}=0.163)$.

We also report a shift of RFs in the direction of attention when both attend

\footnotetext{
${ }^{2}$ For example the motion direction of an outside target stimulus (S3) located close to the horizontal meridian and moving either right or left might be easier for the monkey to be attended and discriminated than the stimuli inside the RF with the same motion direction present somewhere in the right lower visual quadrant. In this case absolute performance level might be generally high but likely lower for the task with attention to the motion direction inside the RF.
} 
inside conditions are compared and thus repeated the previous analysis to test for a relation of performance and RF shifts. Note that the average hit rate difference between the inside conditions was $1.5 \%$ only, but the standard deviation (10.5) and the maximum deviation (37\%) illustrates that there is variability in performance which could be reflected in RF shift variability. To test for its relation with the extent of the RF shift for the inside condition, we correlated the difference of the hit rates with the RF shift. There was, however, no significant correlation of the hit rate differences of the inside condition with the RF shift (measured as the shift of the $\mathrm{RF}$ slice centroids as percentage of the target separation $)(\mathrm{r}=-0.06, \mathrm{p}=0.33)$.

Finally, we also test for a relation of performance differences with changes in RF size. Previous psychophysical research has reported that the width of the attentional focus (measured as the distribution of detection speed near the focus of attention) is narrower with more difficult stimulus discriminability (Handy, Klingstone, and Mangun, 1996; Ahissar and Hochstein, 2000). If attention would be more narrowly distributed around the attended target stimulus, this should have a direct influence on the underlying size of the RFs. We therefore compared changes in (halfheight) RF size when attention was directed outside versus inside the RF with the performance measures used above, i.e. with performance differences and absolute performance levels. The correlation of RF size change and performance difference between the attend outside versus attend inside condition was significant $(\mathrm{r}=-0.17$, $\mathrm{p}=0.037$ ) (cf. figure 3.19). Thus when the monkey attended inside rather than outside the RF, RFs are smaller when performance is better in the inside compared to the outside condition. However, the psychophysical finding suggests that it is the absolute attentional effort that affects the distribution of attentional facilitation. Attentional effort is more directly reflected in absolute performance level and not in performance differences. However, we find that RF size changes were not correlated with changes in absolute hit rate or the amount of fixational errors (all $\mathrm{p}>0.05)$. This latter finding indicates that RFs are not generally smaller with absolutely lower performance level as would be expected from the psychophysical findings (see above). The preceding analysis rests on the assumption that attentional effort is reflected in the average performance level of the monkey in each experiment. It should be noted, however, that attentional effort can only indirectly be inferred from performance measures which are affected by various aspects of the experiment including for example more difficult stimulus conditions (e.g. with higher eccentricity or closer of the stimuli inside the RF, cf. also footnote on page 90 and discussion on p. 106), or differences in motivational state. Furthermore, correlations based on average performance levels (or performance differences) are clearly less sensible than a correlation of neuronal response changes (including the degree of RF size or shift changes) with trial-by-trial variations in reaction time and accuracy, which would 


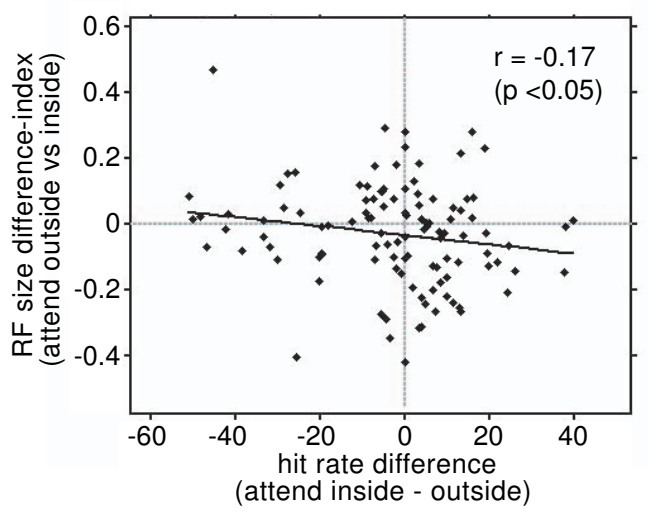

Figure 3.19: Comparison of RF size changes (ordinate) and hit rate difference (abcissa) when the monkey attended outside versus inside the RF. The y-axis plots the RF size index, with negative values reflecting shrinking RFs when attention is directed inside the RF, The abszissa reflects difference of hit rates with high values reflecting better performance when attention is directed inside compared to attention outside the RF. The significant overall correlation of $r=-0.17$ indicates that with a better performance in the attend inside condition, RFs tend to become smaller.

provide a more refined measure of attentional effort. However, our experimental design does not allow a trial-by-trial analysis because the RF parameters (center location or size) require the average of probe responses across many trials.

To summarize, the preceding correlational analysis of RF shifts and RF size changes with absolute hit rates (performance) does not suggest any significant relation but showed if at all (non-significant) trends to stronger shifts and smaller RFs with better performance levels. The only significant effect was a relation of better performance in the attend inside versus the outside condition and smaller RFs in the inside condition, which is opposite to the psychophysically derived expectation of smaller RFs with more difficult tasks, i.e. smaller attentional foci (which relies on the tentative assumption that smaller attentional foci are reflected in smaller RFs). 


\subsection{Time Course of Spatial RF Shift}

The analysis of the spatial sensitivity modulation so far was based on the neuronal response to the RF probes averaged over an interval from 60 to $200 \mathrm{~ms}$ relative to the onset of the RF probe. Averaging spiking activity assumes that the attentional effect on the spatial sensitivity is stable throughout this time period of the presence of the flashed probe stimulus. However, it could well be that the spatial modulation of sensitivity is not present throughout the trial but due to the transient capture of attention by the abrupt luminance and motion onset of the probe stimulus (Yantis and Jonides, 1990; Yantis, 1998; Egeth and Yantis, 1997). According to this view, probe stimuli close to the attended target stimulus would capture attention more effectively than probe onsets more distant to the attentional focus. As a consequence of such an attentional capture, neuronal responses would be affected more strongly for probe locations close to the attended target stimulus. This would create a spatial shift of response strength (or sensitivity) similar to the one that we observe. The two interpretations should be distinguishable on the basis of a temporal analysis of the spatial shift. A putative transient, abrupt onset effect of attentional capture should be evident in a spatial shift of the RF stimulus that is restricted to the later part of the probe response. The on-response phase of the two probe presentations should be less modulated than the sustained, tonic response period, because the attentional effect would be initiated only at the time of probe onset and will be instantiated only with a temporal delay. Such a delayed effect contrasts to a sustained effect of spatial attention which would affect already the first spikes in response to the probe and will also be evident in the earlier on-response time.

We therefore analyzed the time course of the spatial sensitivity shift in response to the RF probe stimuli. For this we computed the RFs for successive 20ms intervals following probe onset and averaged the activity orthogonal to the axis of S1 and S2, i.e. orthogonal to the direction of the attentional shift between the inside condition (the method is indicated in figure 3.20, A). Rather than considering the average stimulus-evoked response to the probe stimulus, we applied a modified version of reverse correlation of the spike times which was triggered on the onset time of the $\mathrm{RF}$ probe stimulus, rather than with regard to the mere presence of the probe stimulus as would be used for conventional reverse correlation. The spatial reverse correlograms are computed in analogy to the spike-triggered averages applied for the direction tuning (cf. figure 2.4, p. 55), but with the stimulus vector reflecting the two spatial dimensions ( $x$ and $y$ ) of the position of the probes, rather than the single dimension (direction) in the direction tuning correlograms. Similar methods with different visual stimulation regimen are widely applied in the analysis of striate and extrastriate visual response properties (cf. e.g. De Angelis and Ohzawa 1993; 

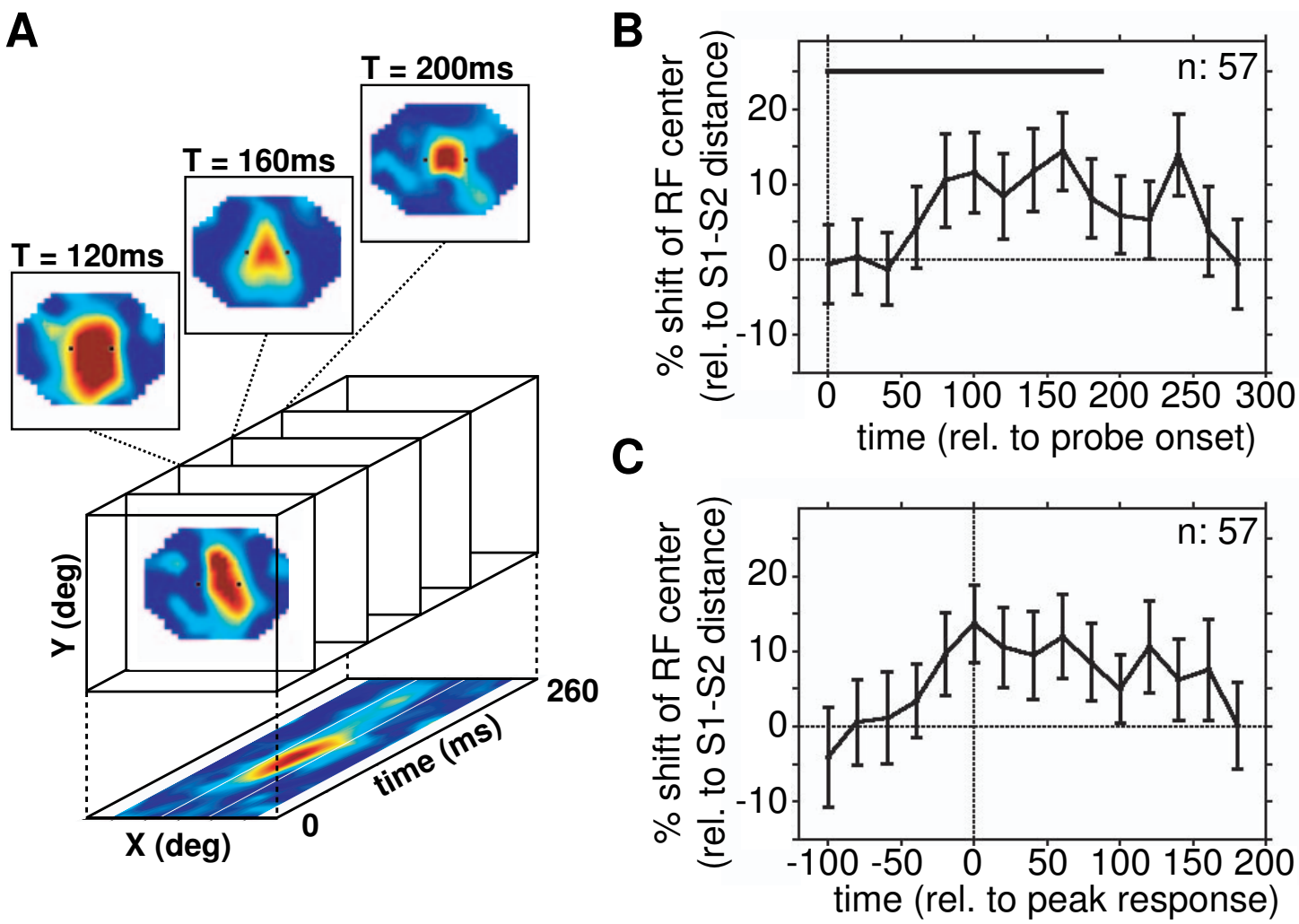

Figure 3.20: Spatial reverse correlation analysis of the time course of the spatial RF shift. A: Two dimensional RFs were obtained for successive $20 \mathrm{~ms}$ bins following probe onset (i.e. at $0 \mathrm{msec}$ ). Activity was then averaged orthogonal to the $\mathrm{X}$-axis to obtain a X-T plot. Stimuli inside the RF always lay along the X-dimension of the plot. The center position of the RF at each time slice was compared between the conditions with attention inside the RF (S1 and S2). B: The spatial RF shift of the attend inside conditions (attend S1 versus attend S2) for different times aligned to the onset of the probe. Positive values indicate a shift towards the attended stimulus. Error bars are the 0.95 confidence limits of the spatial difference of the centroid of the two conditions for the set of 57 cells. The graph shows that RFs are shifted towards the attended stimulus already $80 \mathrm{~ms}$ following probe onset and remain shifted until $240 \mathrm{~ms}$ from stimulus onset. The horizontal bar in the upper right reflects the time of probe presentation. $C$ : Time course of the spatial shift as in $B$ but aligned to the time of the peak response of the correlograms of individual cells. The graph shows that the maximum RF shift is found at the time of the maximum response to the probe stimulus, which shows that it is evident already in the transient on-response to the probes.

De Angelis et al. 1999; Livingstone and Tsao 1999; Livingstone, Pack, and Born 2001).

Figure 3.20 illustrates the resulting $\mathrm{X}-\mathrm{Y}$ RF maps for an example neuron for successive time intervals from probe onset. The spatial layout of the RF maps were always rotated to align the X-dimension parallel to the axis of stimuli S1 and S2 inside the RF. In other words, the shift of attention from S1 towards S2 between the attend inside conditions always ocurred along the $\mathrm{x}$-dimension. In order to 
reduce noise of the correlograms we then averaged activity of the Y-dimension, i.e. orthogonal to the axis of the attentional shift, i.e. of the S1-S2 axis. As a result we obtained an X-versus-Time (X-T) plot representing the probability of observing spikes in response to a probe stimulus at a particular $\mathrm{x}$-position at a particular time from probe onset. The shift of the RF at different times of the X-T plots were obtained in the same were as in the analyis of the RF slices: After obtaining the center of the RF at each time, we computed the spatial difference of the RF center between the two conditions with attention to either S1 or S2, with positive spatial differences indicating that the RF center lies closer to the attended stimulus at that time point. We find that the spatial shift becomes statistically significant already $80 \mathrm{~ms}$ after probe onset and is continuously present until $240 \mathrm{~ms}$ following probe onset (cf. fig. 3.20, B). This time course mimics the sensory latency of the probe response and suggests that the $\mathrm{RF}$ shift is not due to a transient effect induced by the abrupt onset of the probe since this would require an additional delay because of the reaction time to the probe. This is also supported by an additional analysis of the RF shift aligned to the time of the maximum (peak) response to the probe (cf. fig. 3.20, $C$ ). The RF shift is strongest at the time of the maximum probe response, again indicating that the transient on-response of the probes is already spatially modulated by attention. 


\subsection{Eye Position Analysis}

Sensory responses in striate and extrastriate visual cortex including area MT are affected by small fixational eye movements which can induce transient (40ms) surges of neuronal responses even though response variance is typically unchanged by microsaccades (Bair and O'Keefe, 1998; Leopold and Logothetis, 1998; MartinezConde, Macknik, and Hubel, 2000; Snodderly and Kagan, 2001). It is therefore important to control for microsaccades on area MT responses when comparing different experimental conditions. We therefore derived the number and amplitude of microsaccades in the different attentional conditions during the time when RF probe stimuli were flashed in the visual field. Microsaccades were defined as gaze displacements within a $100 \mathrm{~ms}$ time window that exceeded a velocity of $10^{\circ}$ per second ${ }^{3}$. We obtained on average 3.39 microsaccades in conditions with attention inside the $\mathrm{RF}$ with no statistical difference between the attend inside conditions ( $\mathrm{t}$-test, $\mathrm{t}=$ $0.03, \mathrm{p}>0.05)$. While we did observe less microsaccades in the attend outside condition (mean: 2.54) the difference to the attend inside conditions failed statistical significance (t-test, $\mathrm{t}=1.73, \mathrm{p}>0.05$ ). Similarly, the amplitude of microsaccades in the attend inside conditions (mean amplitude: 0.58 and 0.62 , respectively) and in the attend outside condition (mean: $0.60^{\circ}$ ) did not differ significantly (t-tests, all $\mathrm{p}>0.5$, Bonferroni corrected for multiple comparisons). This finding suggests that microsaccadic eye movements did not influence neuronal responses systematically between conditions. In addition to small corrective microsaccades, eye gaze might also drift to different positions within the confines of the small fixation window of $0.6^{\circ}$ radius. Since different foveal positions between conditions will also displace the receptive field (relative to the non-drifting fixation square) we analyzed the average center position of the fovea in the fixation window across conditions. The analysis is

\footnotetext{
${ }^{3}$ We used a sliding window that was shifted by $50 \mathrm{~ms}$ every step and checked for position deviations in time that reflected a velocity of $10^{\circ}$ per second.
}

Figure 3.21: Examples of eye position distribution for attention conditions. $A-C$ : Two dimensional maps with color coding the frequency of the eye position at a particular location relative to the fixation cross (at 0/zero) (Light blue indicates that at least once during the experiment the monky looked at that position). Upper panels illustrate eye positions sampled while the monkey attended that stimulus inside the RF which was presented at a position in the direction from fovea to the red dot plotted on the axis. Lower panels show the same with regard to the condition when the other stimulus was attended which was placed in the direction from fovea to the blue spot on the axis. There was no bias of the eye positions to drift in the direction of the attended target in the examples shown. The distance between the mean eye position of these conditions was small and not related to the extent of the spatial shift in sensitivity. 


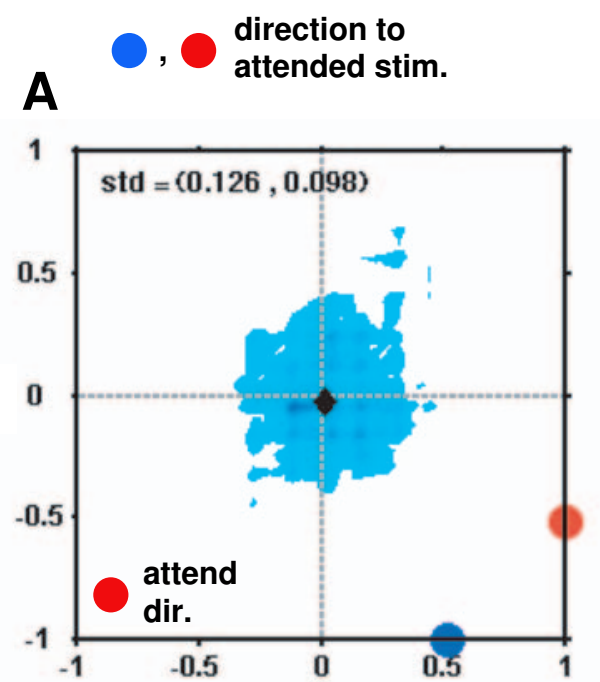

B
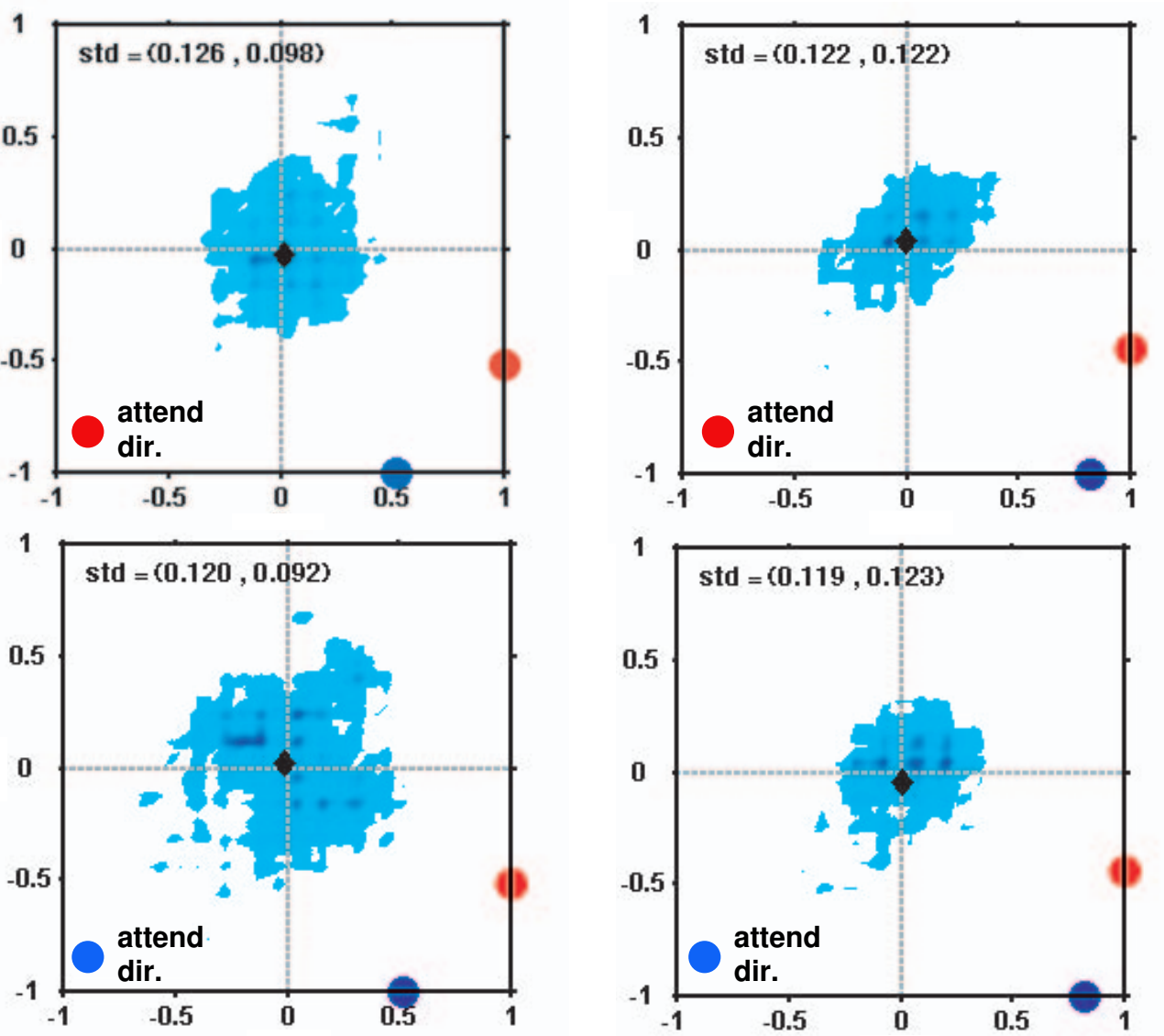

mean eye pos. abs. RF difference: centroid shift: 0.027

$$
\begin{aligned}
& 4.2^{\circ} \\
& 29.2 \% \text { of S1-S2 } \\
& \text { distance) }
\end{aligned}
$$

C
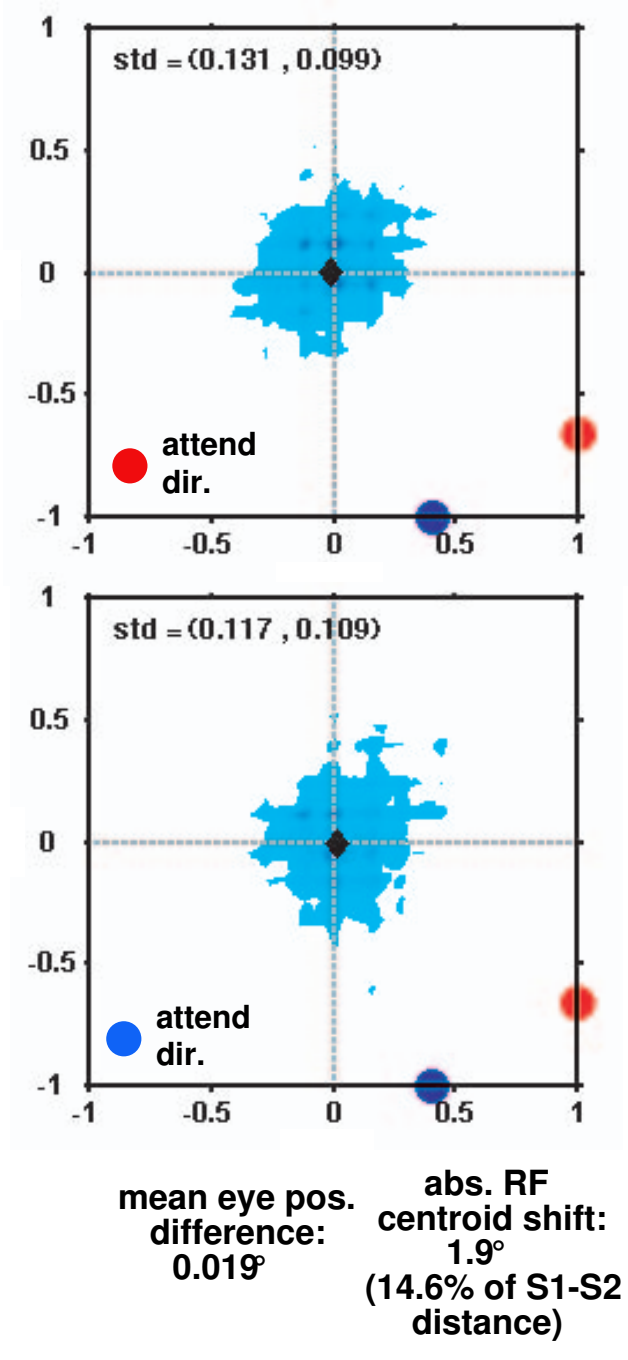
based on two dimensional eye position maps which are illustrated in figure 3.21 (p. 96). The examples show the distribution of eye gaze when attention was directed to either of the stimuli inside the RF (the direction from fovea to S1 and S2 is indicated by the red and blue cicle on the axis). In these examples the center eye position did differ by only $0.019^{\circ}-0.039^{\circ}$ across conditions, and in each case a considerable shift in spatial sensitivity was observed (cf. information at bottom of panels).

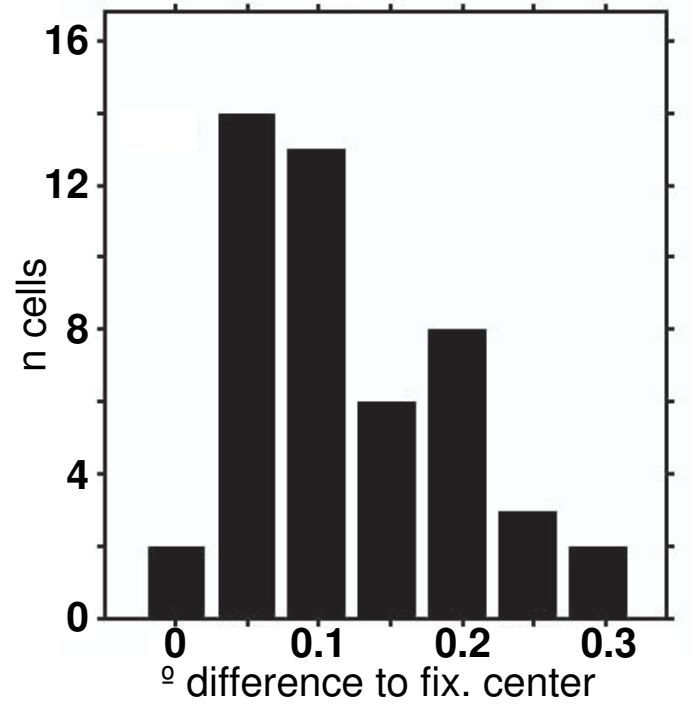

Figure 3.22: Eye position deviation: attend-inside conditions. Distribution of differences in degrees $\left(^{o}\right)$ of the center eye position in trials with attention to $\mathrm{S} 1$ and attention to $\mathrm{S} 2$ inside the RF. observed in the same conditions $(\mathrm{r}=0.20, \mathrm{p}>0.5)$.

Across the population of cells, the scatter of eye positions was on average slighly higher than in the examples with a mean distance between the attend inside condition of $0.12^{\circ}$ (med: $0.10^{\circ}$ ) (standard deviation: of $0.07^{\circ}$ ) (cf. figure 3.22$\left.)\right)^{4}$. The center eye positions in the attend-inside conditions were on average $0.16^{\circ}$ (median: $0.15^{\circ}$ ) offset (std: 0.08) from the eye position centers in the attend out condition. The small difference in eye positions of about $0.1^{\circ}$ contrasts with the far stronger and directionally specific spatial shift of RFs of on average $5.6^{\circ}$ across the population of cells (cf. p. 75). This is also supported by the lack of a significant correlation between eye position deviations of the attend-inside conditions and the proportional RF shift

\footnotetext{
${ }^{4}$ Note, that we had to exclude $n=8$ cells for the eye position analysis due to temporary technical problems with reflections from an unknown noise source (which was evident in extremely high numbers of detected artificial microsaccades evenly in all condition). Inclusion of these cells did not change the overall result (data not shown).
} 


\section{Chapter 4}

\section{Discussion}

The current study investigated the influence of attention on neuronal RFs of well isolated and functionally characterized single neurons in macaque area MT. The following discussion will survey the implications of our results in two main parts. First, findings from the background measurements of direction tuning properties of the studied neurons, and the influence of multiple stimuli in the RF on the sensory RF properties will be discussed. The subsequent discussion will focus on the results concerning our main research question of the influence of attention on the size and position of RFs.

\subsection{Directional Tuning: Spike-Triggered Averag- ing and Classical Methods}

Neurons in the current study were well tuned to the direction of motion. The tuning estimates were derived on the basis of two methods with a high degree of consistency across methods (cf. results section 3.1.1, pp. 59). In particular, the initial tuning estimate which provided the preferred and null direction of the isolated neurons was based on a classical approach involving average firing rate computation to motion in a uniform direction over an extended $(750 \mathrm{msec})$ time period. Interleaved with the attentional mapping experiment, directional tuning was obtained with an adaptation of the recently developed motion reverse correlation technique (Perge et al., 2002; Borghuis et al., 2003). Importantly, the direction tuning with either of the method (and at different times in the experimental session) provided almost identical indices of (i) direction selectivity (average DI of 0.85), (ii) estimates of the preferred direction with Gaussian fits, and (iii) their tuning width (cf. figure 3.1, p. 60). These findings reflect a further validation of the MRC method which has been established only recently in one laboratory for direction tuning in macaque area MT and with a slightly different motion stimulus (Borghuis et al., 2003) and which is 
based on established reverse correlation techniques (e.g. Eckhorn, Krause, and Nelson 1993; Livingstone and Tsao 1999; Livingstone, Pack, and Born 2001; Gonzalez, Perez, and Justo 2001). Notably then, the fine direction tuning with an average DI of about 0.85 and an average Gaussian tuning width of about $48^{\circ}$ is consistent with the functional properties that are expected for the population of area MT neurons (Maunsell and van Essen, 1983; Albright and Desimone, 1987).

Moreover, the successful adaptation of the MRC method in trials that were interleaved with the attentional mapping experiment highlights the quality of single neuron isolation during the course of the experimental sessions: If cell isolation would have been based on multiunit rather than single cell isolation, or if isolation quality would have deteriorated during the course of the experimental session, this would have been recognized in a noisy and less reliable motion reverse correlogram ${ }^{1}$.

\subsection{Sensory RF Maps}

Our attentional mapping experiment required the simultaneous presentation of three stimuli within the RF confines of area MT neurons: the RF probe stimulus moving in the preferred direction of the neuron and two potential target stimuli (S1 and S2). It is therefore critical for the experiment that area MT neurons integrate multiple stimuli in a systematic way. We find that this is the case. In particular, we find that adding non-preferred motion information in the $\mathrm{RF}$ reduces response strength and RF size (cf. result section 3.1.2, pp. 62): Comparing the RF maps in the two sensory conditions which required detection of luminance changes of the fixation square and involved either only probe stimuli (fixation probe condition), or probe stimuli in the presence of S1 and S2 (fixation with S1S2 condition), we find a systematic reduction of RF size (of -19.5\%) and of response strength (of -14.5\%) in the presence of two non-preferred directions of motion. This reduction is less compared to previous studies who reported that pairs of stimuli result in a scaled average response that is roughly half the magnitude of the responses to the individual components of motion (van Wezel et al., 1996; Recanzone and Wurtz, 1997, 2000; Britten and Heuer, 1999; Treue, Hol, and Rauber, 2000). In other words, adding non-preferred direction of motion should have reduced response more than we actually observed. However, the discrepancy to the literature can be easily reconciled by the fact that our probe stimuli had abrupt luminance and motion onsets while S1 and S2 were present continuously throughout the trial. Visual neurons typically have strong transient on-responses compared to a lower sustained response. Since we included the onresponse phase of the probe responses in the analysis (with an analysis window of

\footnotetext{
${ }^{1}$ The quality of the reverse correlogram was used as an additional source for online decisions about whether to accept or reject single neuron isolation for the attentional mapping experiment.
} 
60 - 220msec from probe onset), this should have increased the relative influence of the preferred direction on the neuronal responses compared to the non-preferred direction of motion of stimuli S1 and S2. This is exactly what we found.

However, in contrast to our expectation, the reduction of RF size was not correlated with the reduction of response strength when two stimuli moving in the non-preferred direction were added to the RF. This lack of correspondence points towards a more complex interaction between responses to the individual motion components in the RF on the one hand, and the spatial summation of these components. Consistent with such a non-linearity, Britten and Heuer (1999) reported that the summation of two preferred directions of motion by the population of area MT neurons is best understood as a scaled power law with an exponent that is slightly larger than 0.5. While our data do not allow to conduct such an analysis, our finding highlights non-linear effects such that the addition of two non-preferred motion directions could cause a RF size reduction without response reduction, and vice versa. This finding clearly warrants more detailed investigations of the spatial and feature summation properties of area MT neurons. 


\subsection{Attentional Influence on Spatial Tuning: Sum- mary of Results}

The main finding of the current experiment is a systematic shift of RFs towards the focus of attention on a trial-by-trial basis and under conditions of identical visual stimulation and task-difficulty across attentional conditions. Such a trial-by-trial dynamic of the spatial sensitivity of single neurons in the absence of a systematic shrinkage of RF size with attention to stimuli inside the RF has not been shown before and has various implications for models about the neuronal implementation of selective attention in visual cortex. Moreover, it could reflect a neuronal correlate of various perceptual phenomena of selective attention reported in psychophysics. Before considering these implications a brief summary of the major results will be provided and linked to previous reports of attentional modulation of spatial tuning.

Spatially specific RFs shifts towards the focus of attention

Our study is the first which demonstrates shifts of attention to different subregions within the RF while simultaneously mapping quantitatively RFs. We find that the neuronal RFs shifted systematically in the same direction as the attentional shift (cf. results section 3.3, pp. 71). For the population of cells the neuronal RF shift amounted to one third (center of activity), or even one half (peak-position), of the spatial extent of the attentional shift. The obtained shift was highly systematic and evident in almost all neurons studied. In terms of the size of RFs in conditions when attention was directed inside the RF neurons shifted the center of their spatial sensitivity by 0.18 times the RF size. The RF peak position was shifted 0.29 times the RF size (averaged over attentional conditions) towards the attentional focus (cf. section 3.3.1, p. 72). This result is remarkably similar to findings from area V4 in the ventral processing pathway that is based on a rather different task and stimulus arrangement that utilized a coarse one dimensional RF mapping with bars at five or seven positions evenly spaced by 0.25 times the sensory RF size (Connor et al., 1997). With this design they reported that the peak position of responses to five bars were shifted by 0.1 times the size of RFs when attention was directed to stimuli at opposite ends and outside of the RF of the studied neurons. A stronger peak position shift of 0.25 times the RF was obtained when the attentional targets were placed further apart and seven bars intervened in between. This finding suggested to the authors that RFs tend to shift more when attention is further away from the RF (Connor et al., 1997).

This finding is again reminiscent on our own results. We find that attention brought inside the RF (from the stimulus outside the RF) shifts spatial sensitivity stronger to the attended stimulus the more distant the RF center is in the attend outside condition from that stimulus (cf. figure 3.15, p. 84). This result highlights 
the fact that our task design necessarily underestimates the absolute extent of RF shifts that could follow shifts of the attentional focus. Our task design limited the extent of the attention shift because we forced the potential target stimuli to be located at similarly activating regions of the sensory RF. Stimulus separation was therefore limited by the RF size of area MT neurons. This was less than the target stimuli in the study by Connor et al. (1997), which were placed always outside the $\mathrm{RF}$. We believe that we obtained a shift that is even stronger than that reported by Connor because we required attention to be directed inside the RF, which likely enhanced the spatially specific weighing of responses compared to attention outside the RF. It should be noted, however, that a comparison of the shift extent in our study and Connor et al. (1997) should be treated with caution, because we report the shift relative to the average size of RFs during attentional tasks, while Connor et al. (1997) report spatial changes relative to the size of RFs in a sensory control condition which apparently was not as demanding as their attentional task and thus might have affected RF size and thus RF shift estimates (particularly when reported as proportion of RF size).

In addition to the RF shift towards attended target locations, the previous study by Connor et al. (1997) also suggested a bias of neuronal sensitivity shifts towards the fovea. Our vector analysis of the peak position shift demonstrates that we did not find a foveal bias of RF shifts towards the attended stimulus location. In contrast, we found a (non-significant) trend away from the fovea (cf. results section 3.3.2, pp. 76). We believe that this discrepancy is well explained by differences in stimulus arrangement. Connor et al. (1997) presented target stimuli at varying locations around the fovea depending on the orientation preference of their cells (and because their RFs could lie close to the fovea and targets could be presented at opposite ends of the RF) with the consequence that some target stimuli lay closer to the fovea than others and attentional shifts could follow all kind of directions relative to the fovea. Therefore, attentional shifts to the different target stimuli could already be directed towards and away from the fovea which might account for the foveal bias that they report. In contrast, we directly controlled for this 'confound' of attentional shifts with eccentricity (and also task difficulty) and as a consequence did not find a foveal bias of RF shifts.

Connor et al. (1997) also reported that a subset of neurons showed a spatial sensitivity shift for only one of the two target locations and asserted that neurons might have a specific 'attentional center' of their RFs. However, as discussed in the previous sections, the asymmetry that we found is well accounted for by the separation of the RF in the outside condition and the attended target location in the inside condition. Furthermore, we could show RF shifts to either of two target stimuli within the RF when compared to attention to the stimulus outside the RF. 
These results contradict a fixed neuronal 'attentional center' and rather highlights a high degree of spatial plasticitiy of RF position with varying allocation of attention.

The timing of shifted spatial sensitivity

The spatial change in sensitivity with attention to different subregions could be due to a sustained shift of the RF induced by the cue to selectively focus the target stimulus. Alternatively, it might be due to a transient effect induced by the abrupt luminance onsets of the probes which might have transiently captured attention to the probe and away from the target stimulus inside the RF (Yantis, 1998; Yantis and Jonides, 1990; Egeth and Yantis, 1997; Lamy, Tsal, and Egeth, 2003). Automatic attentional capture should be strongest for probes at positions close to the attended stimulus and thus might have transiently enhanced responses locally around that position. While such an attentional capture would not affect the main finding of an attentional RF shift it would provide a further hint towards likely mechanism. If this scenario would hold true one should expect the spatial change of the RF to be absent in the transient on-response to the probe stimulus, but to arise during the sustained response to the probes. We analysed the temporal development of the spatial shift relative to the probe onset and found that already the first spikes elicited by the RF probe are shifted towards the attentional target (cf. results section 3.5, p. 93). Moreover, the spatial shift was strongest at the time of the maximum response to the $\mathrm{RF}$ probe stimuli, which was typically the peak of the on-response transient. As attentional modulation of the on-response has previously been shown to be weak, and in particular weaker than the modulation of the tonic response period, this finding strongly suggests that the observed shift is due to a sustained state of spatially focused attention rather than to transient capture.

Attention does not shrink RFs systematically

One of the main hypothesis of the project suggests that one of the main mechanisms of attention is a directional specific shift and shrinkage of RFs around attended stimuli (Moran and Desimone, 1985). We find a spatially specific and systematic shift (see above). We also observe a significant, albeit small shrinkage of halfheight RFs with an average geometric mean of the converted size index of $6.2 \%$ (cf. results section 3.3.4, p. 84). However, there was no relation of RF shrinkage to RF shift and we thus conclude that RFs do shift relliably, shrink moderately, but they do not shrink systematically around the attended stimulus inside the RF compared to peripheral attention outside the RF. It should be noted, however, that the spatial shift of RFs does reflect qualitatively the same effect as a concomittant shrinkage: The shift of RF towards the attended stimulus enhances its influence while reducing the influence of the unattended stimulus on responses in visual cortex. However, the absence of RF shrinkage in the presence of a RF shift is in contrast to hypotheses that emphases the role of inhibition to bring about attentional selection. 
A strong influence of attention on inhibitory circuits is at the source of the biased competition hypothesis which hypothesizes that attention gates the propagation of relevant stimulus representation reminiscent to an all-or-none mechanism (c.f. e.g. the title of Moran and Desimone 1985). Instead, the RF shift we report shows that attention gradually routes visual information in favour of stimuli at and close to the attentional focus - which does not necessarily incorporate a primary role of inhibitory circuitry (see below).

RFs shift without changes of response amplitude

Interestingly, the observed spatial shift in the current study occurred in the absence of an attentional effect on the strength of responses to the probes (cf results section 3.2 .2 , p. 68). This finding is based on a two stage analysis of firing rates of the neuron in response to the two potential target stimuli inside the RF (S1, S2) alone, and with the addition of the RF probe. We find that spatial attention inside versus outside the RF enhances responses to the two non-preferred stimuli (S1, S2) by a small but statistically significant $15.7 \%$, but that the addition of the non-preferred $\mathrm{RF}$ probe stimulus does not change this spatial attention effect further, i.e. when we consider the response to the three stimuli (i.e. S1, S2, and probe stimulus) after subtraction of the two-stimulus response without probe stimulus (cf. figure $3.6 \mathrm{~B}$, p. 70).

This result agrees with previous studies. The enhancement of responses when the monkey attended inside versus outside the RF in the presence of two non-preferred stimuli reflects the classical spatial attention effect that is expected to be relatively small with a non-preferred stimulus feature in the RF (McAdams and Maunsell, 1999; Treue and Maunsell, 1996, 1999; Martinez-Trujillo and Treue, 2004). In contrast, the addition of a preferred probe stimulus evokes mechanisms responsible for the push-pull effect of attention, and thus should differentially modulate responses depending on whether a preferred, or non-preferred stimulus feature is attended (cf. section 1.2.4, p. 17). According to the pull-component of the push-pull effect responses will be reduced when attention is directed to a non-preferred stimulus compared to a sensory condition with identical visual stimulation. However, we compared responses to the probes in the attend inside condition to the response when the monkey attended outside the RF to a non-preferred motion direction. In contrast to a sensory control condition our attend outside condition would be expected to already reduce the overall responses of the neuron due to a feature-based attentional influence (Treue and Martinez-Trujillo, 1999; Martinez-Trujillo and Treue, 2004). Therefore, our finding of the lack of additional attentional modulation with the addition of the probe stimulus fits well to reported influences of attention on neuronal responses. In summary, this finding shows that attention induces changes in spatial sensitivity in the absence of changes in response magnitude. 
RF shift does not correlate with task performance

The RF shift we observe is not significantly correlated with measures of task difficulty including performance rates and fixation errors. At first glance, the absence of a relation suggests that the extent of RF shifts is independent of the degree of effort imposed by more difficult task conditions (e.g. when behavioral targets were spatially close together, or at higher eccentricities). However, the absence of a correlation should be treated with caution: Lower hit rates do not always have to be indicative of higher behavioral effort or higher task difficulty, but could also be based on a lower compliance of the monkey with task instructions due to motivational reasons. While we do not have an objective measure of this source of performance rates, this caveat could be one reason of the lack of significant correlations of the $\mathrm{RF}$ shift and hit rate differences in the attentional conditions. It should be noted, however, that all correlations (but one) that were computed had significance values indicating statistical trends (p-values between 0.068 and 0.163 ) towards stronger $R F$ shifts for conditions that were easier, rather than more difficult (cf. results section 3.4, 90). This finding actually contrasts the logic put forth above (suggesting that shifts should be stronger when tasks are more difficult), and rather shows that RFs tend to shift stronger, the more succesfull the monkey was in attending to a particular target stimulus. This reasoning is also consistent with Connor et al. (1997), who found a RF shift with a task that resulted in only $1.5 \%$ errors and which was thus much easier than the one used in the current study which resulted in more than $15 \%$ of errors.

\section{$R F$ size changes and its relation to task performance}

Despite the lack of systematic RF size changes concomitant with the shift of RFs, reductions of $\mathrm{RF}$ size with attention inside compared to outside the RF were moderately, but significantly, correlated with performance rates. Similar to the previous finding of a trend of stronger RF shifts when the monkey performed more accurately, we find that RFs tend to be smaller in the attend inside condition when it is easier than the outside condition. This effect is opposite to expectations derived from psychophysical studies on the size of the attentional focus as a function of task difficulty: It has been shown that the focus of attention is narrower in more difficult, rather than in easier task conditions (see discussion below, pp. 114, and Handy, Klingstone, and Mangun 1996; Ahissar and Hochstein 2000). If the size of RFs is related to the extent of the attentional focus towards the attended stimulus then more difficult tasks should have resulted in a narrower focus and thus a narrower RF. We observed the opposite, which matches with the counterintuitive relation of the RF shift and performance that is apparent as a statistical trend (see above). The apparent contradiction might be solved by the speculative assumption that only more accurate performance in the spatial attention task reflects the suc- 
cessfull deployment of attention and that increasing error rates might be related to a lower compliance with task instructions and an increase in the amount of guessed responses rather than in the amount of effort spent to the task requirements.

\subsection{Neuronal Implications of Spatial Tuning Shifts}

The observed RF shift reflects that neurons become more sensitive to stimuli close to the focus of attention while at more distant locations sensitivity becomes less. Thus, attention exerts a spatially specific influence on the spatial sensitivity of neurons in area MT.

\subsubsection{Attentional Input Modulation and Spatial Routing of Information}

The spatial displacement of RFs towards the attentional focus reflects a change in the preferred spatial position yielding maximum response of neurons in area MT. Such a change in spatial tuning contrasts eith previous findings of a multiplicative interaction of attention and the tuning to motion direction and orientation (McAdams and Maunsell, 1999; Treue and Martinez-Trujillo, 1999): Spatial tuning curves in area MT are not only scaled with spatial attention as are directional tuning curves, but shifted. It is therefore apparent from our finding that selection of spatial positions for efficient processing is based on different, or maybe additional, mechanisms than selection of featural information.

The non-multiplicative shift in spatial tuning could nonetheless be based on multiplicative gain mechanisms when the modulation does not affect the response stage in area MT but presynaptic neurons from areas projecting to area MT (cf. section 1.2 .3 , p. 16). According to this suggestion a spatial weighing towards the attended spatial position could be based on multiplicatively modulated presynaptic responses of neurons with smaller RF that match more closely the size of the attended stimulus (Maunsell and McAdams, 2001). According to this assumption area MT neurons would integrate responses from a population of afferent neurons that is differently affected by spatial attention. The spatial summation of these responses would be based on (multiplicatively) enhanced responses from neurons centered at the attentional focus, which would induce a spatially specific weighing of spatial sensitivity close to that point (Maunsell and McAdams, 2001).

The role of gain modulated $V 1$ responses

This scenario requires that our task design induces attentional modulation in areas that project to area MT and whose RFs are smaller and at a spatial scale that more closely matches the size of the moving RDPs that were the attentional targets in the 


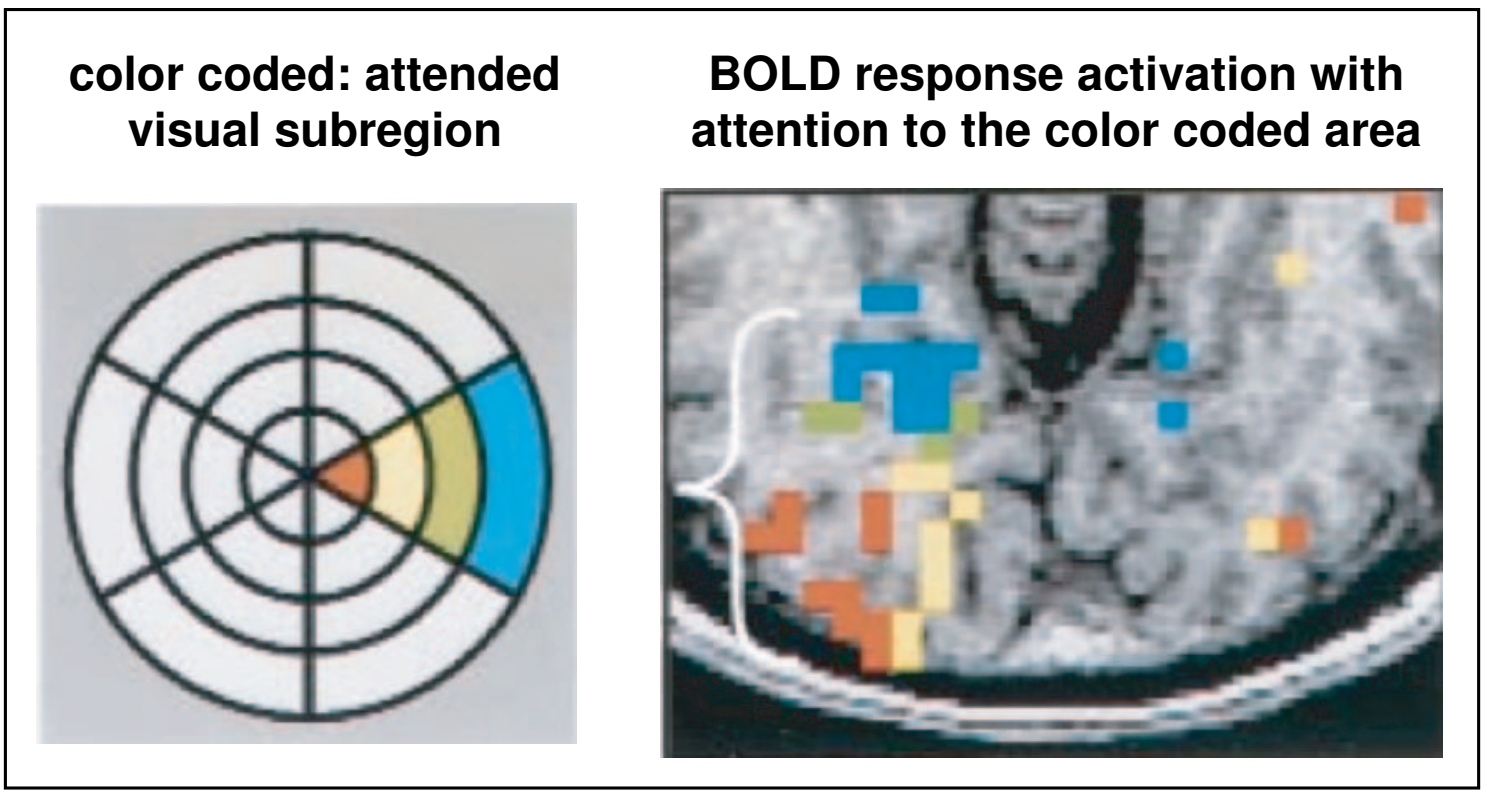

Figure 4.1: Spatial retinotopy of attention in area V1 found in an fMRT study by Brefcynski and DeYoe (1999). When subject attended (compared to passively fixating the center of the display) to the visual field regions indicated by the color (left panel) activity in area V1 (right panel) was modulated retinotopically specific (color of activated regions correspond to color in left panel).

current design. These criteria are met by neurons in striate cortex. In our experimental design the size of the cued RDPs extended between $2-4^{\circ}$ at eccentricities of 8-16 ${ }^{\circ}$ which does fit well to spatial extent of striate neuronal RFs (Press et al., 2001; Angelucci and Levitt, 2002; Levitt and Lund, 2002). Furthermore, spatial attention has been shown to modulate human striate cortex at spatially retinotopic positions overlapping the attentional focus (cf. figure 4.1 and Tootell et al. 1998; Tootell, Tsao, and Vanduffel 2003; Somers, Dale, and Seifert 1999; Brefcynski and DeYoe 1999; Martinez et al. 1999, 2001; Sasaki et al. 2001; McMains and Somers 2004). Moreover, a recent human fMRI study showed particularly strong attentional effects on V1 activity (compared to those in higher areas) when a small region in space had to be attended rather than larger areas (Müller et al., 2003). Also noteworthy is the observation that attentional modulation in area V1 is often contingent on a stimulus arrangement that places the attentional target in the presence of distracting information - similar to the arrangement in our task (e.g. Martinez et al. 1999; Noesselt et al. 2004). Several earlier studies without contextual stimulation failed to observe attentional modulation within V1 (cf. Noesselt et al. (2004), and references therein). A similar observation is also valid for single neuron recording in area V1: Strong top-down effects are observed on single neuron activity when attention had to be directed to a stimulus embedded within contextual stimuli (Motter, 1993; Vidyasagar, 1998; Ito and Gilbert, 1999; Christ and Li, 2001; Marcus and Van Essen, 2002; McAdams and Reid, 2003), while effects with single attended stimuli were 

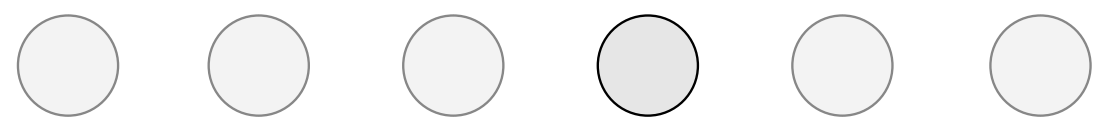

are a MT

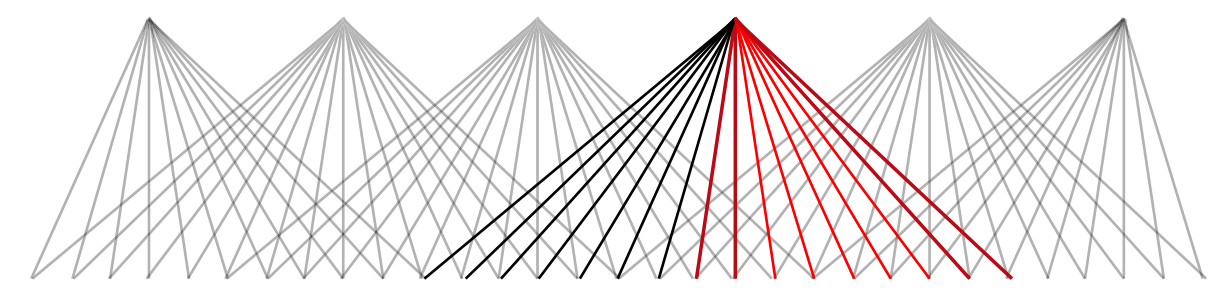

0000000000000000000000000000000

\section{are a V1}
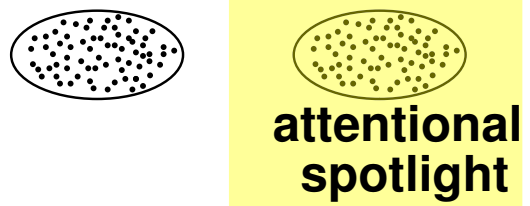

visual

Figure 4.2: Illustration of the putative effect of the attentional spotlight that covers one of two stimuli in the receptive field (bottom part of the figure). The attentional influence will modulate responses in an area with RFs that match closely the size of the attended stimulus (such as area V1). The spatially specific modulated responses will be propagated to higher areas (such as area MT) and will cause a shift in the spatial sensitivity profile of these neurons.

considerably smaller (McAdams and Maunsell, 1999), or absent (Luck et al., 1997b).

The finding of stronger modulation in task designs with complex stimulus arrays might well be due to an interaction of the attentional influence with contextual influences of area V1 neurons. Almost all neurons within area V1 do have suppressive regions surrounding the excitatory $\mathrm{RF}$ centers which enhance local feature contrast in a bottom-up fashion (Polat, Mizobem, and Kasamatsu, 1998; Polat, 1999; Kapadia and Westheimer, 1999; Levitt and Lund, 2002). The design deployed in the current experiment will have activated these bottom-up mechanisms since attended RDPs and probes always moved in opposite directions. Since attentional modulation is particularly evident with such displays area V1 neurons likely contribute to the observed effects of attentional shifts of spatial sensitivity.

Taken together, this brief survey shows that area V1 neurons are affected by attention in tasks with nearby distracting stimuli and thus provide the initial stage of selective propagation of these signals throughout cortex. Attention routes spatial information not only by the enhanced processing of stimuli within the attentional spotlight but also by reducing the influence of unattended stimuli on efferent areas. The observed shift of spatial sensitivity would accordingly reflect an orderly spatially pooling of an already modulated retinotopic map of an afferent neuronal population. The proposed neuronal mechanisms of a feedforward projection of gain modulated responses is illustrated in figure 4.2 .

Input integration in area $M T$ is spatially specific 
The influence of striate cortical activation on responses of area MT neurons might also be inferred indirectly from recent adaptation studies. Kohn and Movshon (2003) reported that adaptation effects on neuronal responses can be specific to subfields of the RFs of area MT neurons. Prolonged adaptation of RF subregions induced a decrease in contrast gain (by a factor of three) that was restricted to the adapted subfield. According to the authors the spatial specificity of their finding should be due to adaptation occurring within striate cortex with neuronal RFs matching the extent of the adaptation stimulus (Kohn and Movshon, 2003). This suggestion resembles the proposed mechanisms underlying the attentional RF shift that we observe. We find reduced gain far from (and enhanced gain near to) the attended focus. This similarity raises the question whether adaptation and attention share a common neural mechanisms that is evoked with opposite signs: Adaptation reduces sensitivity, while attention (typically) enhances it. We are, however, not argueing that adaptation played a role for the RF shift: The reported adaptation effect was based on prolonged stimulation (of fourty seconds) that is not comparable to our stimulus timing. Reduced adaptation durations of about three seconds have been shown to have low or even no net effect on area MT neurons (van Wezel and Britten, 2002). Furthermore, short-term adaptation effects, causing an initial transient onresponse to level off to a sustained tonic firing rate, have been shown to affect responses in area MT spatially non-specific across the RF of the MT neurons (Priebe, Churchland, and Lisberger, 2002). In summary, long-term adaptation effects in area MT support the assumption that the influence of afferent neuronal populations is spatially specific. According to this view the observed RF shift with attention is due to spatially specific modulation at the input stage.

\section{Alternative accounts: inhibitory interactions}

In contrast to a feedforward model of gain modulated neuronal responses, the spatial RF shift could also be based on mechanisms that are consistent with the biased competition account. Reynolds and Desimone (1999) suggests that spatially pooled responses in area $\mathrm{V} 4$ will recurrently inhibit input activity that are driven by stimuli outside the focus of attention. While Reynolds and Desimone (1999) proposed this mechanism to support the RF shrinkage hypothesis, it also appears to be generally applicable to spatial shifts in the absence of RF shrinkage (e.g. when considering the spatial width constants of the inhibitory and excitatory effects). However, it should be re-emphasized that our data do not reveal a particular role of inhibitory compared to facilitatory effects of attention. The proposed mechanism would rely on attentional signals that affect inhibitory interneurons with a spatial grain corresponding to the size of the attentional spotlight. Moreover, this mechanistic scenario - as the gain mechanism proposed above - needs to be investigated in computational simulation studies which would help in specifying and constraining the underlying 
neuronal architecture of attentional effects.

The computational modeling is particularly relevant also in the light of inhibitory mechanisms that have been suggested to account for plasticity of RFs that goes along with recovery from cortical lesions, artifical scotoma conditioning, and learning (cf. section 1.3, pp. 32). Cortical models that are motivated by findings in this line of research, also including area MT (cf. Sober, Stark, and Yamasaki 1997), often take recourse to interareal inhibitory mechanisms. A recent, rather general model of visual processing has likewise successfully implicated a coarse interareal inhibitory pool of neurons to model attentional effects, but did not extend it to attentional changes of spatial tuning (cf. Rolls and Deco 2002) ${ }^{2}$. In summary, our finding of a $\mathrm{RF}$ shift in the absence of systematic RF size changes will likely contribute to the specification of the underlying neuronal architecture. Computational models of the RF shift will be capable to foster new experimental designs for a further clarification of the underlying mechanisms of attentive vision.

\subsubsection{Attentional RF Recruitment, Cortical Magnification, and Spatial Resolution}

The attentional shift of spatial sensitivity towards attended locations reflects the recruitment of neuronal RFs close to the focus of attention: the attended focus will be sampled by neuronal RFs that would otherwise represent more distant locations. As a consequence, space is sampled with a higher density which essentially corresponds to a local enhancement of cortical magnification and for covert eccentric attention - reducing the discrepancy of lower peripheral and higher foveal magnification. Thus, RF recruitment will likely increase the spatial resolution at and near attended locations which will be evident in enhanced spatial selectivity of the population response.

An analog assertion has recently been made in an fMRI study which required subjects to attend to rotated three dimensional objects after prolonged adaptation to similar views of the behavioral target (O'Murray and Wojciuluk, 2003). These authors found that attention appeared not only to enhance the BOLD response (in human lateral occipital cortex), but additionally activates a more specific population of neurons as was evident in the degree of non-overlapping BOLD responses for shapes of different rotation when they were attended compared to non-attended (O’Murray and Wojciuluk, 2003).This finding suggested to the author that attention

\footnotetext{
${ }^{2}$ A classical model of RFs that shift their spatial positions that has not been mentioned is the shifter circuit by Olshausen, Anderson, and van Essen (1993). This model suggests that a special set of shifter neurons which re-route information at progressing stages of processing remaps RFs to different input populations dependent on the position of attention. While this model would likely reproduce the result of area MT RF shifts, its architecture appears not to be biologically plausible and is thus not discussed in more detail.
} 
sharpens the population of neurons recruited to encode the behavioral target feature. A similar sharpening of the population response likely follows from our finding of the spatial shift of RFs.

\section{Enhanced spatial sensitivity}

The various implications of the proposed recruitment and spatial sharpening of the population response in area MT is illustrated in figure 4.3 (see below and figure legend for details). The local enhancement of cortical magnification should result in an increase in spatial sensitivity and acuity of visual (here: motion) processing at attended locations. Various psychophysical studies have shown that attention indeed increases sensitivity in various task contexts deploying suprathreshold stimuli such as small vernier stimuli, Landolt squares, and orientation contrasts or near threshold gratings (Hawkins et al., 1990; Henderson, 1991, 1996; Luck et al., 1996; Müller et al., 1998; Carrasco and Yeshurun, 1998; Carrasco, Williams, and Yeshurun, 2002; Yeshurun and Carrasco, 1999, 2000; Cameron, Tai, and Carrasco, 2002). Moreover, the attentional benefit of searching for targets within arrays of distracting elements is stronger for peripheral cues than more foveal cues (Carrasco and Penpeci-Talgar, 2000). These findings could be an explicit consequence of the illustrated recruitment of neurons near attentionally focused regions. Our results of RF shifts could provide the likely neuronal basis for such an effect: sensitivity of single neurons shifts closer to attended locations. The next section will follow this assumed consequence of our study in more detail. 


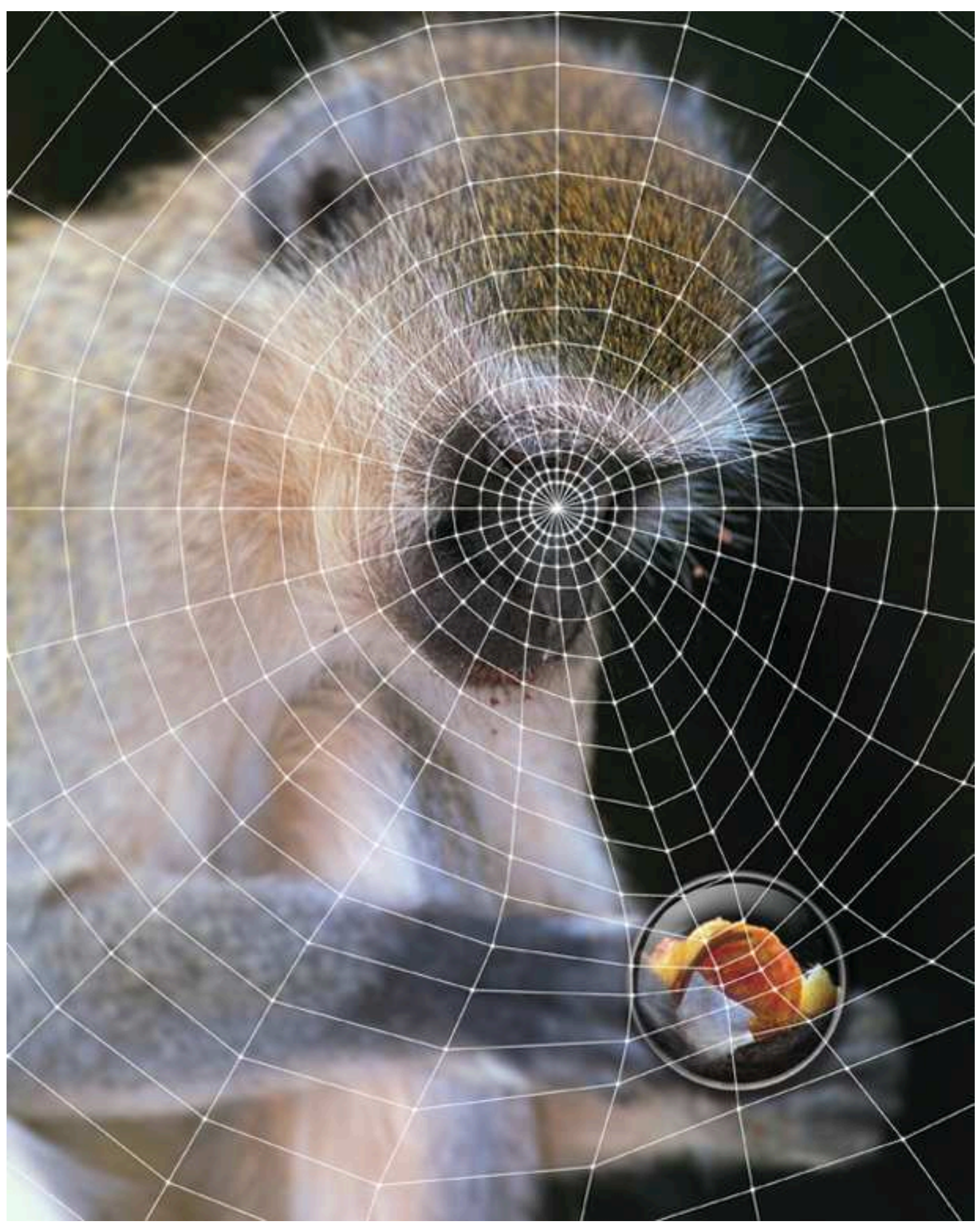

Figure 4.3: The figure illustrates the hypothetical effect of attention on the neuronal representation of an attended spatial location in extrastriate visual cortex when gaze is directed to the vervet monkeys face and peripheral attention is allocated to the fruit in her hand. The nodes of the white net reflect the center of neuronal RFs with the highest density of neurons at the fovea and a gradual decrease of cortical magnification in the periphery. Peripheral spatial attention attracts neuronal resources (RFs) toward the attended spatial position. The first consequence of peripherally focused attention is an increased number of neurons encoding features at the attended location (the region in the lower right) which implies a reduction of the difference in cortical magnification from periphery and fovea (the center of the circular net). The second major consequence would be a distortion of the spatial representation at and near the focus of attention in extrastriate visual cortex (see text for details). 


\subsection{Perceptual Implications of Attentional RF Re- cruitment}

The current project finds that spatially facilitated processing of a target RDP is paralleled by a shift of the activation gradient of neuronal RFs toward the attended target. In other words, attention recruits neuronal sensitivity (or neuronal resources in general) towards the focus of attention. Such a spatial shift of neuronal sensitivity could be the neural correlate of various perceptual effects of attention, including (i) distributions of enhanced processing accuracy close to the attentional focus and suppression in its surround, (ii) enhanced spatial resolution, and (iii) distortions in directional, distance, and other spatial judgements. In the following the implication of our main finding of the RF shift on these psychophysical aspects will be discussed.

\subsubsection{Spatial Distributions of Attention}

Spatial attention enhances the efficiency of visual processing of stimuli overlapping the attended region, while information outside the focus of attention is often processed worse when compared to conditions without focal attention. Attentional distributions of facilitation and suppression has been found by many studies using reaction time, sensitivity $\left(d^{\prime}\right)$, accuracy measures and employing precuing, visual search or whole report tasks (Shulman, Wildon, and Sheehy, 1985; Eriksen and St.James, 1986; Hughes and Zimba, 1985; Downing and Pinker, 1985; Downing, 1988; Tsal and Lavie, 1988; LaBerge, 1995, 2000; Kim and Cave, 1995). Psychophysical studies have shown that the spatial distribution of facilitatory and suppressive effects of attention can be highly variable in extent and shape. This flexibility of attention has given rise to various analogies of the spatial attentional focus such as (i) a zoom lens of variable size and resolution, (ii) an adjustable spotlight (or beam) with a rather discrete boundary of its focus, or (iii) an attentional window (Eriksen and St.James, 1986; Posner, Snyder, and Davidson, 1980; Tsotsos et al., 1995; Treisman and Gelade, 1980). All these hypotheses assume a particular spatial gradient, or distribution of attentional facilitation close to the focus of attention and reduced visual sensitivity in its surround (Cave and Bichot, 1999).

However, empirically observed distributions vary widely with regard to the range and extent of facilitation and suppression. The basic pattern within this heterogenity is facilitation close to the focus of attention and suppression at surrounding regions which dissipates gradually at various extents to eventually reach a baseline level of efficiency. This pattern resembles therefore mexican hat distributions which are based on the difference of an excitatory and an inhibitory Gaussian (DOG). Such a DOG distribution is well captured by the spatial attractor model sketched in 


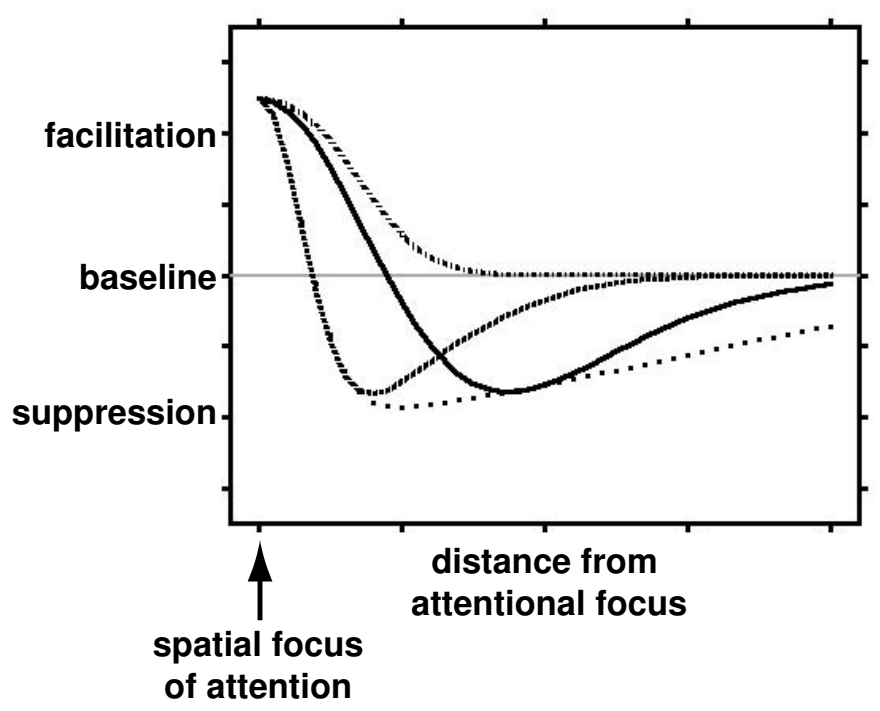

Figure 4.4: Putative sketch of various forms of spatial gradients observed in psychophysics. Attending to a circumscribed region in space (at $\mathrm{x}=0$ in the figure) has been reported to induce changes in the processing efficiency in the surround of this focus of attention. The DOG functions depicted in the figure sketches putative spatial distributions of facilitation (positive y-values), suppression (negative $y$-values), and a plateau of background processing efficiency. While the form of these distributions has been derived from reported gradients in the literature (see text for details), it should be noted that empirical studies have never attempted to match observed gradients with parameters of a difference of Gaussians (e.g. extent and gain of excitatory and inhibitory Gaussian).

figure 4.3 (p. 113), with the center of facilitation corresponding to the attentional focus. Since the outlined sketch is thought to reflect the consequence of attentionally induced RF shifts on cortical magnification and spatial representation, the resemblance with the reported psychophysical spatial gradients suggest a tight functional link of both, neurophysiological and psychophysical phenomena. In particular, the RF shift observed in the current study could be the source of the psychophysically observed spatial DOG gradient of attention. It should be noted explicitly that the suggested functional coupling of the neuronal and psychophysical attentional gradients has not been demonstrated and remains speculative. However, the suggested link might provide specific hypotheses for future approaches about the mechanisms of spatial attention and could contribute to resolve existing controversies of the spatial distribution of attention.

These controversies basically concern the form and extent of the gradient of attention. While there is general agreement of facilitation within the focus of attention, the effect on processing stimuli in the surround ranges from a gradient of pure facilitation (dash-dotted line in figure 4.4), to exclusive suppressive effects in the surround, peaking close to the focus of attention (dotted line in figure 4.4). Studies 
reporting the former extreme of a pure facilitatory effect of attention has been conducted by LaBerge and colleagues (LaBerge, 1995; LaBerge et al., 1997). In their studies subjects had to monitor two letter strings in succession for a predefined target letter. The target position of the first displays was thought to set the position and size of the attentional focus before the second display was presented. Detection speed for the target in the second display was found to be fastest close the position of the target in the first display and leveled off gradually with increasing distance to that position (LaBerge, 1983; LaBerge and Brown, 1989).

In contrast to this task other studies reported a cost, or suppression, of processing stimuli presented at positions away from the focus of attention. In these studies focus of attention was manipulated with the use of uninformative peripheral precues, abrupt onset stimuli, or feature singletons, or in dual task paradigms with the use of a primary task at a fixed spatial position. With these tasks detection, or discrimination performance (response speed or accuarcy) decreases with increasing distance of the attentional focus (Downing and Pinker, 1985; Henderson and Macquistan, 1993; Handy, Klingstone, and Mangun, 1996). Studies deploying these tasks have reported distributions that do reveal facilitation that is exclusively limited to the region of a salient stimulus with inhibition for all stimuli surrounding that stimulus (Henderson, 1991; Henderson and Macquistan, 1993; Handy, Klingstone, and Mangun, 1996; Cave and Zimmerman, 1997; Mounts, 2000b; Collie et al., 2000; Cutzu and Tsotsos, 2003). In these studies target detection is slowed and impaired at all but the cued position, which could be located already $2-3^{\circ}$ away from the attentional focus (Bahcall and Kowler, 1999; Handy, Klingstone, and Mangun, 1996; Downing, 1988; Müller and Humphreys, 1991). The degree of suppression is sometimes reported to be strongest close to the attentional focus and to dissipate very slowly with increasing distance to the focus of attention, sometimes without to asymptote to background performance level even at more distant positions (cf. dotted line in figure 4.4, Cave and Zimmerman 1997; Caputo and Guerra 1998; Mounts 2000b,a; Cutzu and Tsotsos 2003). For example, Mounts (2000b) reported in a set of studies exclusive neighbourhood suppression of accuracy and detection speed of targets in the vicinity of a color singleton that automatically captured spatial attention. In this study subjects had to discriminate the form of a predefined target shape in the presence of multiple distracting elements of similar shape (Mounts, 2000b).

\section{Mechanisms of spatial gradients of attention}

The decline in accuracy and response speed close to a colored element in the display was suggested to be due to an active inhibitory mechanism that is strongest close to the attended stimulus (Mounts, 2000b), that is thought to follow mechanisms proposed by the spatial ambiguity resolution hypothesis (Luck et al., 1997b). This hypothesis transfers the RF-shrinkage hypothesis into the psychophysical domain 
by claiming that inhibition should affect only the processing of stimuli that are presented within the confines of spatial receptive fields of neurons coding for that stimulus. According to this assumption competitive interactions should shrink RFs around the attended stimulus and filter other stimuli that would otherwise result in responses of the neuron (cf. section 1.2.4, p. 19). As already noted in the previous section, our finding of a $\mathrm{RF}$ shift has qualitatively similar effects to an $\mathrm{RF}$ shrinkage. However, in contrast to RF shrinkage, the RF shift that we observe does not necessesarily imply inhibitory interactions to bring about an enhanced influence of stimuli at the attentional focus. Our finding suggests therefore a more gradual spatial effect of attention that does not result in the strongest suppression in the immediate vicinity of the attentional focus, but a gradual drop of facilitated processing towards true suppression at distant locations to the attentional focus.

The spatial gradients could accordingly be accounted for by a gradual routing of processing resources that is brought about by a shift of (maybe fixed amount) of neuronal resources without the requirement of an active inhibitory component. Such a gradual shift of resources is visualized as the displacement of nodes in figure 4.3 (p. 113), rather than a deletion of nodes at distant parts. This gradual routing of neuronal resources has already been used to explain spatial gradients of attention that included facilitatory and inhibitory aspects (Bahcall and Kowler, 1999). However, this gradual approach might well account also for reported spatial distributions with peak suppression for stimulus positions next to the attentional focus, if it is assumed that a facilitatory component would emerge when processing would be probed for locations closer to the attentional focus than has been done in these studies. This suggestion calls upon methods that map attentional effects with a higher spatial density and resolution than previous studies (which typically used less than six distances). Recent studies have begun to obtain such high resolution maps of attention (Ahissar and Hochstein, 2000; Bennett and Pratt, 2001; Tse, Sheinberg, and Logothetis, 2003) and future studies will be needed to clarify this issue further.

\subsubsection{Spatial Distortions with Focal Attention}

The attentional shift of neuronal RFs reflects a change in their preferred spatial location. Since neuronal RFs in area MT convey positional information by means of retinotopically organized RFs, spatial attention induces a distortion of that topography. As a consequence, tasks which rely on spatial signals from area MT neurons should be affected by spatial attention. In particular, displacement of the RF profile towards the attended location results in stronger responses near the attended location and less response to stimuli presented at the position formerly occupied by the RF center (i.e. in the absence of spatial attention). According to this rea- 
soning psychophysical studies have shown that stimuli (small oriented vernier lines) are judged to be farther away from a position when that position was attended, as if the stimuli are encoded by neurons which would otherwise encode more distant locations (Suzuki and Cavanagh, 1997). Such a spatial repulsion effect might well be explained by the spatial distortion induced by shifts of spatial sensitivity at the level of individual RFs as found in the the current project. A similar mislocalization of flashed stimuli has been reported for briefly flashed stimuli, which are consistently located towards more peripheral locations (Tsal and Bareket, 1999; Musseler et al., 1999). This finding might again reflect an encoding of these stimuli by RFs that would represent more peripheral locations in the absence of attention. However, it should be emphasized that this reasoning relies on the assumptions that spatial and distance judgements in these tasks are based on retinotopically organized areas and that their information is not corrected for attentional shifts.

The proposed tentative link of attentional RF shifts and psychophsycial performance might also give rise to predictions about the extent of the expected spatial effects. The previously described spatial repulsion effect was observed for stimuli that were far away $\left(>5^{\circ}\right)$ from the primary attentional target. This finding fits nicely with our result of a gradual RF shift and with previous reports of spatial RF shifts with attention to regions outside the RF (Connor et al., 1997). However, very close to the attentional focus spatial localization might actually be biased towards, rather than away from the attentional focus, if it is assumed that cortical magnification is locally enhanced. Indirect evidence for this assumption might be lend from a study showing that lines are perceived shorter at the focus of attention than they are physically (Tsal and Shalev 1996, but cf. Prinzmetal and Wilson 1997). If contraction of line-length is due to a constriction of the spatial representation underlying line representation then this finding provides strong evidence for the spatial distortion of space with attention as indicated in figure 4.3 (p. 113). In addition to line length judgements locally enhanced magnification with focal attention would also predict influences on size judgements as described above.

\subsubsection{Spatial Resolution of Attention}

The influence of RF shifts on local cortical magnification at peripherally attended locations also shows that spatial attention reduces the difference of peripheral and foveal representation capacity. The psychophysical consequence might be derived from visual search studies showing a greater benefit of cuing for peripheral over central target positions (Carrasco and Yeshurun, 1998). Furthermore, a higher sampling density within the attentional focus might also enhance the spatial resolution of visual processing which is also suggested by psychophysical reports (He, Cavanagh, 
and Intriligator, 1996; He and Cavanagh, 1997; Yeshurun and Carrasco, 1998, 2000). Yeshurun and Carrasco (1998) showed that segmentation of textures of fixed size is facilitated at peripheral locations while performance was impaired for more foveal locations. The authors assumed that the texture was optimally sized for RFs of a particular size. Accordingly attention could have reduced the size of the RFs which would be beneficial for RFs that would be larger than the texture (as in the periphery) while detrimental near the fovea (with already small RFs) ${ }^{3}$.

\subsection{General Summary and Conclusion}

This project reports a systematic spatial sensitivity shift that follows the focus of attention - on a trial-by-trial basis and at identical eccentricities and under identical task difficulty - with various approaches: The RF shift was evident in the comparison of conditions with attention to different subregions inside the RF (inside-inside comparison, sect. 3.3.1) and with attention to subregions inside versus attention outside the RF (inside-outside comparison, sect. 3.3.2). Furthermore the position change of the RFs was evident in the center of mass and peak position of one dimensional projections of the RF activity profile (slice analysis), as well as in the peak position of the two dimensional RF maps. Additional analysis of the shift showed that there was no bias of the shift towards the fovea (vector analysis, sect. 3.3.2). This analysis also showed that RFs shifted towards the stimulus inside the condition with no bias towards the stimulus outside the RF, showing that RFs shift to the inside stimulus when attended and towards the outside stimulus when that stimulus is attended, which replicates the attention shift reported by Connor et al. (1997).

Moreover, in contrast to an asymmetric attentional RF shift towards only one 'fixed positon' of the RF, which would be suggestive of a static 'attentional RF center' of single neurons (Connor et al., 1997), we showed that asymmetries in the extent and direction of RF shifts are due to stimulus arrangements and that RFs show a strong directional specificity: RFs shifted more towards an attended stimulus the further away they were from that stimulus in the attend outside condition (sect. 3.3.3). Further direct implication for underlying mechanisms can be derived from our temporal analysis of the response to the RF probe stimulus, which showed that

\footnotetext{
${ }^{3}$ Attention has also been reported to be generally more limited in spatial resolution than 'retinal' vision (Intriligator and Cavanagh, 2001). It should also be noted, that some authors (Intriligator and Cavanagh, 2001) suggest that attention is generally limited in spatial resolution. This claim is based on the suggestion that the difference of 'individuation' of a particular item (e.g. are there two or three lines in the visual field) and the detection of the presence of items (e.g. are there lines present in the visual field) is followed by a difference of attentive versus non-attentive processes. We do not follow this reasoning and thus do not try to link it with our main neurophysiological finding.
} 
sustained peripheral attention induces sustained shifts of RFs, in contrast to possible transient effects due to the abrupt luminance onset of the probe stimulus (sect. 3.5).

Our finding of a small marginally significant shrinkage of RFs with attention inside the RF (sect. 3.3.4) complicates the general picture somewhat with regard to the possible underlying mechanisms of attention involving inter-neuronal competition, or multiplicative gain modulation of afferent neuronal populations. However, the moderate shrinkage of RFs was not related to the systematic shift of RFs (sect. 3.3.4), nor was it related to modulation of neuronal response strength (sect. 3.3.5).

In summary, the project was able to reveal that selective attention gradually routes information flow by partial shifts of receptive fields towards the attentional focus. The reported finding is grounded on well characterized and controlled behavioral conditions and neuronal responses: First, neurons were confirmed to be located in area MT based on histological reconstruction (sect. 2.1.2) and functionally inferred from strong and consistent tuning to the direction of motion with two independent methods (sect. 3.1.1). Secondly, the behavioral performance, i.e. the focus of attention, was constant across conditions and ensured an equal task difficulty level (sect. 3.2.1). Thirdly, directional specificity of the RF probe responses were not confounded by eye position shifts (sect. 3.6). Fourth, we reproduced the basic and classical spatial attention effect of stronger neuronal responses with attention inside compared to outside the RF - when we considered the response to the stimuli inside the RF in the absence of the RF probe stimulus (sect. 3.2.2). This last aspect is particularly noteworthy because we obtained the RF shift in response to the probe stimulus in the absence of additional attentional modulation of response strength to the probe.

These findings lead to several general conclusions: First, RFs shift towards the focus of attention without equally strong systematic RF size changes. Such a gradual shift with enhanced activity close to the focus of attention and reduced sensitivity to probes far from it is consistent with a gain hypothesis of attention that assumes attentional modulation to act on the responses of afferent, presynaptic neuronal population. Accordingly, area MT neurons would integrate already modulated responses from neurons with smaller receptive fields that closely match the spatial extent of the attended stimuli (such as in primary visual cortex) either by a feedforward, or a recurrent mechanism. While the functional consequence of the shift is qualitatively consistent with the effect expected from the RF shrinkage hypothesis, it does not reflect an exclusive gating mechanism with an emphasis on inhibitory mechanisms that a shrinkage around attended stimuli implies and which is often insinuated as spotlight, or beam metaphors of selective spatial attention. More clearly, we do not observe a shrinkage around the attended stimulus.

The reported shift of single neuronal spatial sensitivity in extrastriate area MT 
reflects at the population level the additional recruitment of neuronal resources at the focus of attention. This mechanism could be the neural correlate of various perceptual effects that are centered on the focus of attention, including (i) distributions of enhanced processing accuracy close to the attentional focus and suppression in its surround, (ii) enhanced spatial resolution, and (iii) distortions in spatial judgements.

Overall, this project revealed for the first time a high degree of spatially specific plasticity of neuronal RFs due to selective visual attention with implications for models about the mechanisms of attention and about the relation of perceptual phenomena and modulations at the level of single cortical neurons. 


\section{Appendix A}

\section{Difference Map Examples}

Illustration of difference maps similar to the demonstration figure 3.8 on page 73 . The following figure A.1 shows a further example of the individual RF maps when $\mathrm{S} 1$ and $\mathrm{S} 2$ is attended and the resulting difference map.

Figure A.2 (p. 125), A-H, shows examples of difference maps obtained by subtracting normalized RF maps from conditions when S2 (blue diamond) was attended and when S1 (green diamond) was attended. RF maps were rotated to bring the S2 position always to the right on the horizontal plane. A spatial shift of sensitivity towards the attended stimulus position is evident by enhanced activity (red to yellow color code) close to S2 and negative values (blue to white) close to S1. Note that the color axis always covers the same range which reveals whether neuronal activity is asymetric in the two attentional conditions (as in examples $C$ and $G$ ). Note that the difference maps in $G$ and $H$ are based on a spatial arrangement of the probe positions with a smaller separation between the attended S1 and S2 stimulus compared to the other maps. In all examples, the spatial difference of activity follows direction of the attentional shift from $\mathrm{S} 1$ to $\mathrm{S} 2$ and is roughly orthogonal to the S1-toS2 axis. 

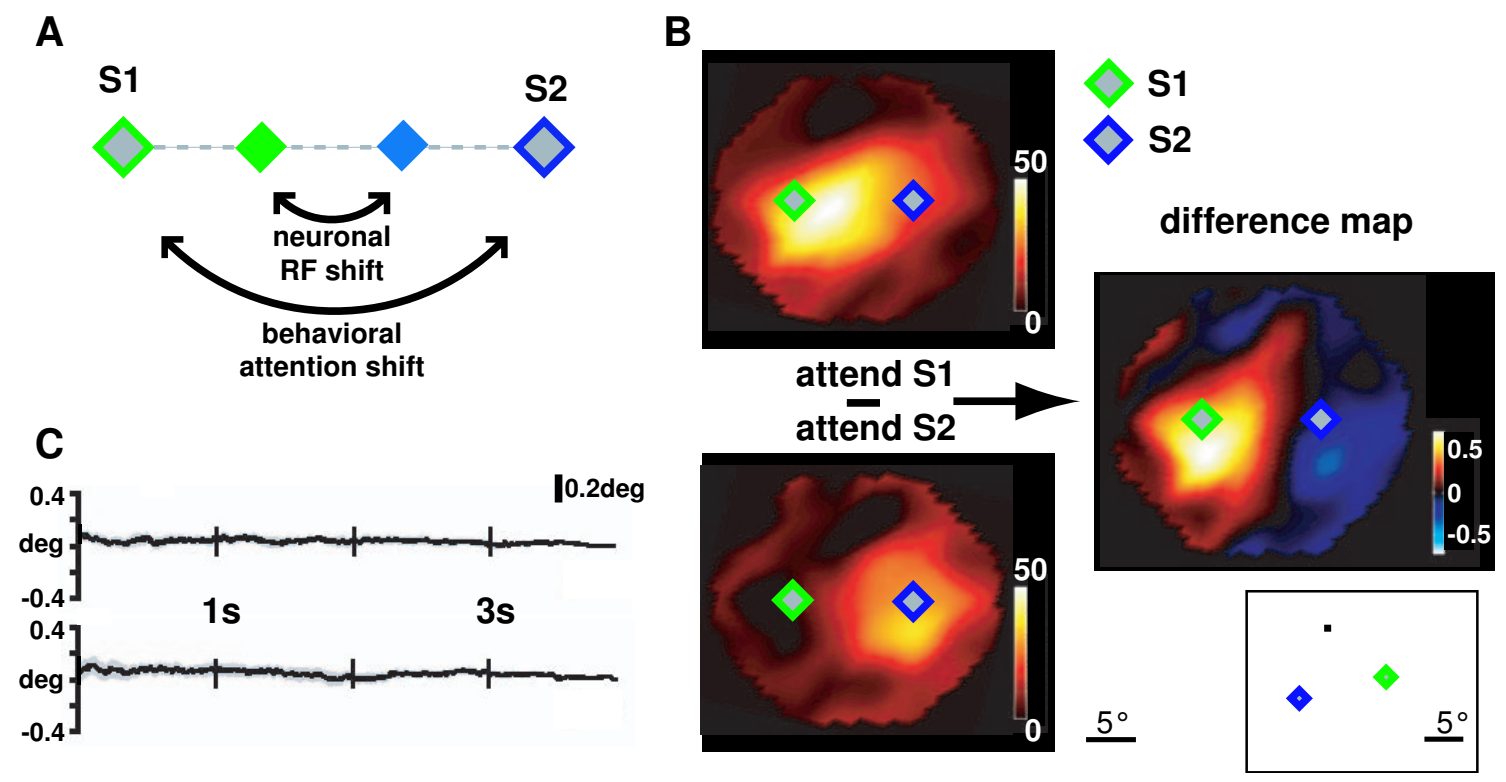

Figure A.1: Illustration of difference map computation, identical to that shown in figure 3.8 (p. 73) but based on a different cell. $A$ : Sketch of the method to quantify shifts in neuronal sensitivity when attention is directed to either of the stimuli inside the RF. The behavioral shift of attention follows the outer diamonds (stimulus positions), while the center of neuronal actitivity (centroid of the RFs, indicated by colored diamonds) is expected to shift on the same axis and in the same direction as the behavioral shift. $B$ : Example of receptive field maps obtained while attention was directed either to the stimulus in the left (top panel, indicated as green bordered diamond), or to the right subregion of the receptive field (bottom panel). Maps are rotated to bring the S1-S2 axis on the horizontal. The neuron illustrates enhanced spatial sensitivity close to the attended stimulus. Responses are stronger to probes close to the attended stimulus while responses were reduced distant to the focused stimulus region. The difference map (right panel) illustrates that spatial sensitivity shifted along the axis of the attended stimuli. $C$ : Illustration of the average spatial deviation of the eye position (fovea) from the fixation point across trials in the condition when S1 was attended (upper panel) and when S2 was attended (S2). The time axis starts at the beginning of the first probe onset and continues throughout the period in which RF probe responses were obtained. Small grey shading shows the upper and lower 0.95 confidence interval of the eye traces. The traces show that foveal position remains almost constant within a small spatial range with a mean distance of the eye position traces between both conditions of $0.057^{\circ}$, which makes it unlikely that eye position has a strong effect on the RF displacement shown in $B$. 


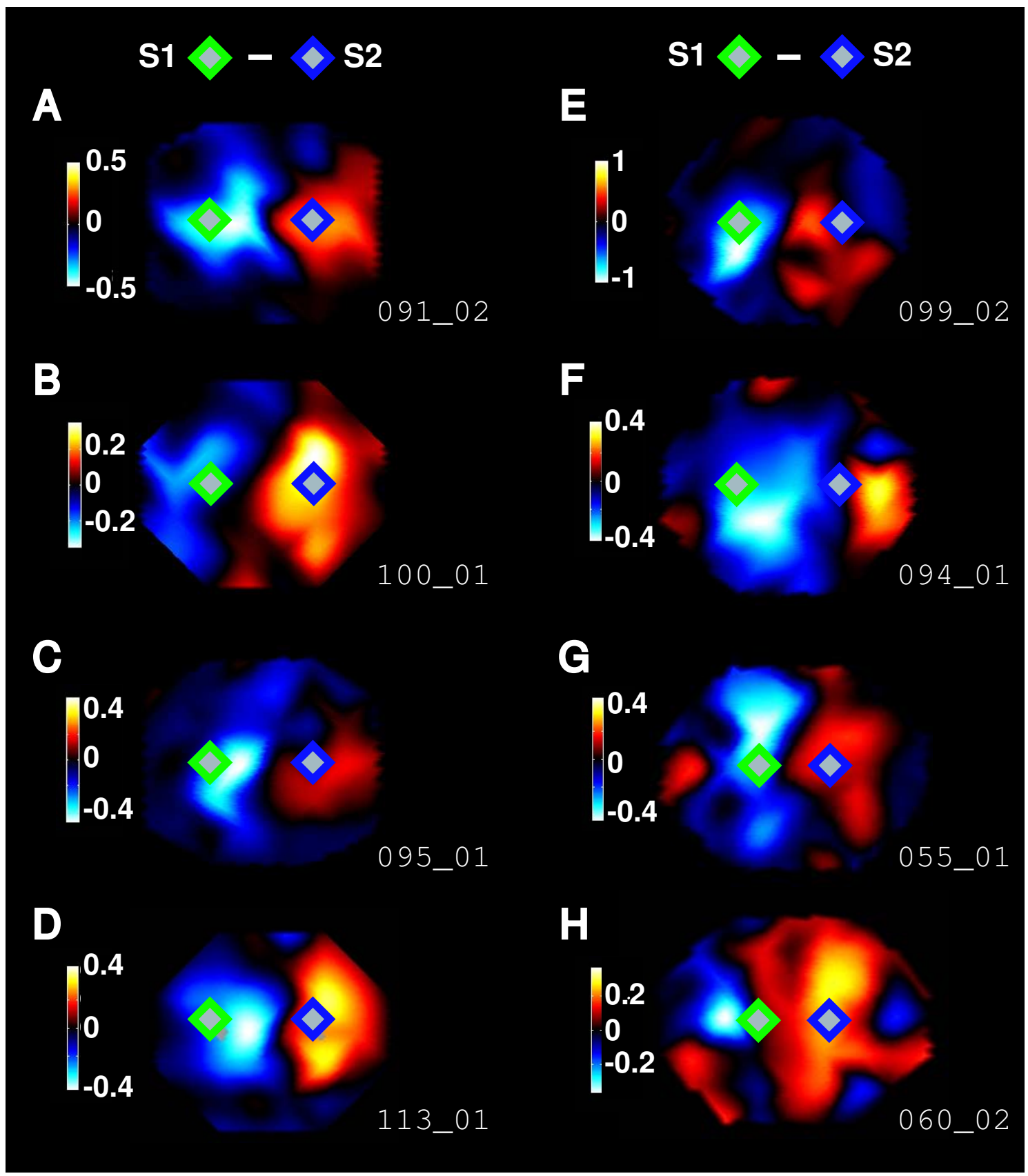

Figure A.2: Example of difference maps. See text for details. 


\section{Appendix B}

\section{Recorded Cells: Overview of Experiments}

The attentional mapping experiment and the tuning experiment was done in a subset of all neurons that could be isolated. An overview of all neurons that were recorded from and the experiments conducted on each of them is given in the following table B.1. Note that most neurons were not used for the experiment simply because they did not match the requirements (for a full list of exclusion criteria, cf. descriptions for column 15 in table B.1). The following will provide information about each column of the table. Please contact the author for more detailed information.

1. cell: Number of cell recorded in chronological order. Note that a cell describes an islated unit which coul be composed of a single cell, or of multiunit activity (cf. column 7). Each isolated unit can contribute to more than one experiment.

2. dataFile: Name of the experiment in a custom format. Experiments which contain the string tune $G$ or tune $A$ contain conditions for the classical direction (and speed) tuning and tests of tuning to more complex optic flow motion patterns which were analyzed for sections 3.1.1 (p. 59). Experiments which contain the string $A M$ contained conditions for the attentional mapping experiment and could also contain further experimental protocols which are not discussed in this project (see below for further details).

3. area: Area of the isolated neuron inferred from functional and anatomical reconstruction (cf. p. 46, section 2.1.2). The star indicates cases which were assigned to an area but likely lay at the border to neighbouring areas such as area MST or V4t.

4.-5. xGrid, yGrid: $x$ Grid and $y$ Grid reflect the position of electrode penetration in the recording chamber in mm relative to the center of the chamber (which was $8 \mathrm{~mm}$ posterior to the AP-0 level and $13 \mathrm{~mm}$ lateral from midline, cf. p. 46). 
Note that positioning could vary from the positions by $\pm 0.5 \mathrm{~mm}$, because we could rotate the chamber grid by $\pm 45^{\circ}$ so that the provided coordinates only give a general orientation (please contact the author for exact coordinates).

6. depth: Depth of recording (i.e. of electrode tip) relative to the dura. Note that these values are approximations and subject to slight variations.

7. isolation: Unit isolation was divided in three levels, single unit (su), mostly single unit $(m s u)$, and multi-unit $(m u)$, depending on how selective neuronal action potentials could be isolated.

8. DI: Direction Index obtained in the experiment with the motion reverse correlation method (cf. methods section 2.3 , p. 54).

9. n: Average number (n) of probe stimuli presented at each location of the virtual grid spanned across the RF of the neuron during the mapping experiment (in the attention conditions). The number gives an indication of the signal-tonoise ratio of the maps with higher values generally providing lower standard errors.

10. dir. tuning: Indication of whether the experiment contributed to the analysis of directional tuning with the classical and the spike-triggered average approach as outlined in section 3.1.1 (pp. 3.1.1). For this and the following columns entries of 1 shows that the datafile was considered for the analysis, while 0 reflect non-usage.

11. RF gain: Indication of whether the experiment has been used for the analysis of attentional effects on response strength (cf. section 3.2.2).

12. slice shift: Indication of whether the experiment has been used for the analysis of the RF shift of the centroids and peak locations of the one dimensional RF slices (cf. sections 3.9 to 3.11).

13. vect. shift: Indication of whether the experiment has been used for the analysis of shift direction of the RF peak position relative to an ideal shift vector (cf. section 3.11).

14. RF size: Indication of whether the experiment has been used for the analysis of size changes with attention (cf. section 3.16).

15. note on exclusion: 
MU (multiunit isolation): Poor isolation of single neuron response with action potentials from other cells contributing to the isolation. This category was also applied to isolations that initially were restricted to a single unit but changed during the course of an experiment.

noBaseline: Missing baseline response needed for the analysis of the RF maps

LowN: Low number of trials for experimental conditions. Typically LowN reflects that the monkey did not perform enough trials and stopped performing before enough data were collected. At other times there were technical difficulties including problems with the recording hardware, the eye-position control, or with the custom software for stimulus presentation and behavioral control.

ScatRF: Scattered (i.e. heterogeneous) receptive field profile in the sensory mapping condition with fixation and no S1 and S2 stimulus inside the RF.

EccRF: receptive field is either too small and foveally, or too eccentric for the attentional mapping experiment.

noExp: recording was done without collection of data for the attentional mapping experiment. Typically slight variations of the experiment were tested with these datafiles.

nDir: not directionally tuned, which also could entail noisy bilobed tuning functions indicative of orientation tuning, or a lack of responsiveness to moving random dot patterns compared to sweeping oriented bars.

noResp: unit that could not be driven by a RF probe stimulus moving in the preferred direction of the cell in the presence of two stimuli moving in the non-preferred direction (S1 and S2). 
Table B.1: List of recorded cells and experiments done which each of them. See text for details about columnar information.

\begin{tabular}{|c|c|c|c|c|c|c|c|c|c|c|c|c|c|c|}
\hline $\begin{array}{l}1 \\
\text { cell }\end{array}$ & $\begin{array}{l}2 \\
\text { dataFile }\end{array}$ & $\begin{array}{c}3 \\
\text { area }\end{array}$ & $\begin{array}{l}4 \\
\mathrm{x}\end{array}$ & $\begin{array}{l}5 \\
\mathrm{y}\end{array}$ & $\begin{array}{c}6 \\
\text { depth }\end{array}$ & $\begin{array}{c}7 \\
\text { isol. }\end{array}$ & $\begin{array}{c}8 \\
\mathrm{DI}\end{array}$ & $\begin{array}{l}9 \\
\mathrm{n}\end{array}$ & $\begin{array}{l}10 \\
\text { dir } \\
\text { tun. }\end{array}$ & $\begin{array}{l}11 \\
\mathrm{RF} \\
\text { gain }\end{array}$ & $\begin{array}{l}12 \\
\text { slice } \\
\text { shift }\end{array}$ & $\begin{array}{l}13 \\
\text { vect. } \\
\text { shift }\end{array}$ & $\begin{array}{l}14 \\
\mathrm{RF} \\
\text { size }\end{array}$ & $\begin{array}{l}15 \\
\text { note on exclusion }\end{array}$ \\
\hline 1 & two-tuneG-rau-001-01+01 & MT* $^{*}$ & -1 & 3 & 6282 & $\mathrm{su}$ & - & - & 0 & 0 & 0 & 0 & 0 & - \\
\hline \multirow[t]{2}{*}{2} & two-tuneG-rau-002-01+01 & - & 1 & -1 & 12585 & $\mathrm{su}$ & - & - & 0 & 0 & 0 & 0 & 0 & - \\
\hline & two-AM-rau-002-01+01 & - & 1 & -1 & 12585 & $\mathrm{su}$ & - & - & 0 & 0 & 0 & 0 & 0 & - \\
\hline \multirow[t]{3}{*}{3} & two-tuneG-rau-003-01+01 & - & 1 & 3 & 10891 & $\mathrm{su}$ & - & - & 0 & 0 & 0 & 0 & 0 & - \\
\hline & two-tuneG-rau-003-01+02 & - & 1 & 3 & 10891 & $\mathrm{su}$ & - & - & 0 & 0 & 0 & 0 & 0 & - \\
\hline & two-AM-rau-003-01+02 & - & 1 & 3 & 10891 & $\mathrm{su}$ & - & - & 0 & 0 & 0 & 0 & 0 & LowN \\
\hline 4 & two-tuneG-rau-004-01+01 & - & 5 & 0 & 9488 & $\mathrm{su}$ & - & - & 0 & 0 & 0 & 0 & 0 & nDir \\
\hline 5 & two-tuneG-rau-005-01+01 & - & 1 & 0 & 9500 & $\mathrm{mu}$ & - & - & 0 & 0 & 0 & 0 & 0 & MU, nDir \\
\hline \multirow[t]{3}{*}{6} & two-tuneG-rau-005-02+01 & - & 1 & 0 & 9582 & $\mathrm{mu}$ & - & - & 0 & 0 & 0 & 0 & 0 & - \\
\hline & two-AM-rau-005-02+01 & - & 1 & 0 & 9582 & $\mathrm{mu}$ & - & - & 0 & 0 & 0 & 0 & 0 & LowN \\
\hline & two-AM-rau-005-02+02 & - & 1 & 0 & 9582 & $\mathrm{mu}$ & - & - & 0 & 0 & 0 & 0 & 0 & noExp \\
\hline \multirow[t]{3}{*}{7} & two-tuneG-rau-007-01+01 & $\mathrm{MT}^{*}$ & 2 & 1 & 16160 & $\mathrm{su}$ & - & - & 0 & 0 & 0 & 0 & 0 & nDir \\
\hline & two-AM-rau-007-01+01 & $\mathrm{MT}^{*}$ & 2 & 1 & 16160 & $\mathrm{su}$ & - & - & 0 & 0 & 0 & 0 & 0 & LowN \\
\hline & two-AM-rau-007-01+02 & $\mathrm{MT}^{*}$ & 2 & 1 & 16160 & $\mathrm{su}$ & 0.70 & 11 & 1 & 0 & 0 & 0 & 0 & noBaseline \\
\hline 8 & two-tuneG-rau-008-01+01 & - & 4 & -2 & 8700 & $\mathrm{msu}$ & - & - & 0 & 0 & 0 & 0 & 0 & - \\
\hline 9 & two-tuneG-rau-008-02+01 & - & 4 & -2 & 10322 & $\mathrm{su}$ & - & - & 0 & 0 & 0 & 0 & 0 & - \\
\hline \multirow[t]{2}{*}{10} & two-tuneG-rau-009-01+01 & - & 3 & -2 & 10260 & $\mathrm{msu}$ & - & - & 0 & 0 & 0 & 0 & 0 & MU \\
\hline & two-AM-rau-009-01+01 & - & 3 & -2 & 10260 & $\mathrm{msu}$ & - & - & 0 & 0 & 0 & 0 & 0 & LowN \\
\hline \multirow[t]{2}{*}{11} & two-rau-tuneG-010-01+01 & - & 3 & -1 & 8460 & $\mathrm{msu}$ & - & - & 0 & 0 & 0 & 0 & 0 & - \\
\hline & two-rau-AM-010-01+01 & - & 3 & -1 & 8460 & $\mathrm{msu}$ & - & - & 0 & 0 & 0 & 0 & 0 & LowN \\
\hline \multirow[t]{2}{*}{12} & two-tuneG-rau-011-01+01 & - & 3 & -3 & 8076 & $\mathrm{su}$ & - & - & 0 & 0 & 0 & 0 & 0 & - \\
\hline & two-AM-rau-011-01+01 & - & 3 & -3 & 8076 & $\mathrm{su}$ & 0.37 & 20.9 & 1 & 0 & 0 & 0 & 0 & noBaseline \\
\hline 12 & two-tuneG-rau-012-01+01 & - & 4 & 0 & 8150 & $\mathrm{mu}$ & - & - & 0 & 0 & 0 & 0 & 0 & MU \\
\hline \multirow[t]{2}{*}{13} & two-tuneG-rau-013-01+01 & - & 2 & -2 & 10005 & $\mathrm{msu}$ & - & - & 0 & 0 & 0 & 0 & 0 & MU \\
\hline & two-AM-rau-013-01+01 & - & 2 & -2 & 10005 & $\mathrm{msu}$ & 0.94 & 6.1 & 1 & 0 & 0 & 0 & 0 & noBaseline \\
\hline 14 & two-tuneA-rau-014-01+01 & - & 2 & -2 & 11950 & $\mathrm{su}$ & - & - & 0 & 0 & 0 & 0 & 0 & MU \\
\hline 15 & two-tuneA-rau-015-01+01 & - & 3 & -4 & 10650 & $\mathrm{su}$ & - & - & 0 & 0 & 0 & 0 & 0 & $\mathrm{nDir}$ \\
\hline
\end{tabular}


... Table B.1 continued

cell dataFile area $\mathrm{x} \quad \mathrm{y}$ depth isol. DI $\mathrm{n}$ dir $\mathrm{RF}$ slice vect. RF note on exclusion

\begin{tabular}{|c|c|c|c|c|c|c|c|c|c|c|c|c|c|c|}
\hline \multirow[t]{2}{*}{16} & two-tuneA-rau-016-01+01 & $\mathrm{MT}^{*}$ & 1 & 0 & 6039 & $\mathrm{su}$ & - & - & 0 & 0 & 0 & 0 & 0 & - \\
\hline & two-AM-rau-016-01+01 & $\mathrm{MT}^{*}$ & 1 & 0 & 6039 & $\mathrm{su}$ & 0.92 & 11.5 & 1 & 0 & 0 & 0 & 0 & noBaseline \\
\hline \multirow[t]{3}{*}{17} & two-tuneA-rau-017-01+01 & - & 3 & 0 & 1890 & $\mathrm{su}$ & - & - & 0 & 0 & 0 & 0 & 0 & - \\
\hline & two-PLX-rau-017-01+01 & - & 3 & 0 & 2100 & $\mathrm{su}$ & - & - & 0 & 0 & 0 & 0 & 0 & LowN \\
\hline & two-PLX-rau-017-01+01 & - & 3 & 0 & 2100 & $\mathrm{su}$ & - & - & 0 & 0 & 0 & 0 & 0 & LowN \\
\hline \multirow[t]{4}{*}{18} & two-tuneA-rau-018-01+01 & - & 3 & -3 & 4460 & $\mathrm{su}$ & - & - & 0 & 0 & 0 & 0 & 0 & - \\
\hline & two-tuneA-rau-018-01+01 & - & 3 & -3 & 4460 & $\mathrm{su}$ & - & - & 0 & 0 & 0 & 0 & 0 & - \\
\hline & two-AM-rau-018-01+01 & - & 3 & -3 & 4460 & $\mathrm{msu}$ & - & - & 0 & 0 & 0 & 0 & 0 & MU \\
\hline & two-AM-rau-018-01+01 & - & 3 & -3 & 4460 & $\mathrm{msu}$ & - & - & 0 & 0 & 0 & 0 & 0 & MU \\
\hline \multirow[t]{2}{*}{19} & two-tuneA-rau-018-02+01 & - & 3 & -3 & 5030 & $\mathrm{su}$ & - & - & 0 & 0 & 0 & 0 & 0 & nDir \\
\hline & two-tuneA-rau-018-02+01 & - & 3 & -3 & 5030 & $\mathrm{su}$ & - & - & 0 & 0 & 0 & 0 & 0 & nDir \\
\hline \multirow[t]{2}{*}{20} & two-tuneA-rau-019-01+01 & - & 3 & -2 & 7317 & $\mathrm{su}$ & - & - & 0 & 0 & 0 & 0 & 0 & LowN \\
\hline & two-tuneA-rau-019-01+01 & - & 3 & -2 & 7317 & $\mathrm{su}$ & - & - & 0 & 0 & 0 & 0 & 0 & LowN \\
\hline 21 & two-tuneA-rau-020-01+01 & $\mathrm{MT}^{*}$ & -2 & 1 & 5426 & $\mathrm{su}$ & 0.68 & - & 0 & 0 & 0 & 0 & 0 & - \\
\hline 22 & two-tuneA-rau-021-01+01 & - & 2 & -3 & 8070 & $\mathrm{msu}$ & - & - & 0 & 0 & 0 & 0 & 0 & nDir \\
\hline 23 & two-tuneA-rau-021-02+01 & - & 2 & -3 & 8690 & $\mathrm{msu}$ & - & - & 0 & 0 & 0 & 0 & 0 & MU \\
\hline \multirow[t]{2}{*}{24} & two-tuneA-rau-021-03+01 & - & 2 & -3 & 8640 & $\mathrm{msu}$ & - & - & 0 & 0 & 0 & 0 & 0 & - \\
\hline & two-AM-rau-021-03+01 & - & 2 & -3 & 8640 & $\mathrm{msu}$ & - & - & 0 & 0 & 0 & 0 & 0 & MU, ScatRF \\
\hline 25 & two-tuneA-rau-022-01+01 & - & 2 & -4 & 6490 & $\mathrm{msu}$ & - & - & 0 & 0 & 0 & 0 & 0 & - \\
\hline 26 & two-tuneA-rau-023-01+01 & - & 4 & -3 & 9100 & $\mathrm{msu}$ & - & - & 0 & 0 & 0 & 0 & 0 & - \\
\hline 27 & two-tuneA-rau-023-02+01 & - & 4 & -3 & 9260 & $\mathrm{su}$ & - & - & 0 & 0 & 0 & 0 & 0 & LowN, nDir \\
\hline \multirow[t]{5}{*}{28} & two-tuneA-rau-024-01+01 & - & 4 & 0 & 3375 & $\mathrm{su}$ & 0.84 & - & 0 & 0 & 0 & 0 & 0 & - \\
\hline & two-tuneA-rau-024-01+01 & - & 4 & 0 & 3375 & $\mathrm{su}$ & 0.82 & - & 0 & 0 & 0 & 0 & 0 & - \\
\hline & two-AM-rau-024-01+01 & - & 4 & 0 & 3375 & $\mathrm{su}$ & - & - & 0 & 0 & 0 & 0 & 0 & - \\
\hline & two-AM-rau-024-01+01 & - & 4 & 0 & 3375 & $\mathrm{su}$ & - & - & 0 & 0 & 0 & 0 & 0 & LowN \\
\hline & two-AM-rau-024-01+02 & - & 4 & 0 & 3375 & $\mathrm{su}$ & - & - & 0 & 0 & 0 & 0 & 0 & LowN \\
\hline 29 & two-tuneA-rau-025-01+01 & - & 3 & -2 & 7590 & $\mathrm{mu}$ & 0.79 & - & 0 & 0 & 0 & 0 & 0 & MU \\
\hline
\end{tabular}


... Table B.1 continued

area $\mathrm{x} \quad \mathrm{y}$ depth isol. DI $\mathrm{n}$ dir $\mathrm{RF}$ slice vect. RF note on exclusion tun. gain shift shift size

\begin{tabular}{|c|c|c|c|c|c|c|c|c|c|c|c|c|c|c|}
\hline & two-tuneA-rau-025-01+01 & - & 3 & -2 & 7590 & $\mathrm{mu}$ & 0.90 & - & 0 & 0 & 0 & 0 & 0 & EccRF, MU \\
\hline \multirow[t]{2}{*}{30} & two-tuneA-rau-025-02+01 & - & 3 & -2 & 7970 & $\mathrm{mu}$ & 0.89 & - & 0 & 0 & 0 & 0 & 0 & MU \\
\hline & two-tuneA-rau-025-02+01 & - & 3 & -2 & 7970 & $\mathrm{mu}$ & 0.82 & - & 0 & 0 & 0 & 0 & 0 & MU \\
\hline \multirow[t]{2}{*}{31} & two-tuneA-rau-025-03+01 & - & 3 & -2 & 9200 & $\mathrm{mu}$ & 0.83 & - & 0 & 0 & 0 & 0 & 0 & MU \\
\hline & two-tuneA-rau-025-03+01 & - & 3 & -2 & 9200 & $\mathrm{mu}$ & 0.88 & - & 0 & 0 & 0 & 0 & 0 & MU \\
\hline \multirow[t]{4}{*}{32} & two-tuneA-rau-025-04+01 & - & 3 & -2 & 9750 & $\mathrm{msu}$ & - & - & 0 & 0 & 0 & 0 & 0 & nDir \\
\hline & two-tuneA-rau-025-04+01 & - & 3 & -2 & 9750 & $\mathrm{msu}$ & - & - & 0 & 0 & 0 & 0 & 0 & nDir \\
\hline & two-tuneA-rau-025-04+02 & - & 3 & -2 & 9750 & $\mathrm{su}$ & - & - & 0 & 0 & 0 & 0 & 0 & nDir \\
\hline & two-tuneA-rau-025-04+02 & - & 3 & -2 & 9750 & $\mathrm{su}$ & - & - & 0 & 0 & 0 & 0 & 0 & nDir \\
\hline 33 & two-tuneA-rau-026-01+01 & - & 0 & -1 & 8290 & $\mathrm{su}$ & - & - & 0 & 0 & 0 & 0 & 0 & LowN \\
\hline 34 & two-tuneA-rau-027-01+01 & - & -1 & -2 & 9700 & $\mathrm{su}$ & 0.96 & - & 0 & 0 & 0 & 0 & 0 & MU \\
\hline \multirow[t]{2}{*}{35} & two-tuneA-rau-027-02+01 & - & -1 & -2 & 10090 & $\mathrm{msu}$ & - & - & 0 & 0 & 0 & 0 & 0 & LowN \\
\hline & two-tuneA-rau-027-02+01 & - & -1 & -2 & 10090 & $\mathrm{msu}$ & - & - & 0 & 0 & 0 & 0 & 0 & - \\
\hline 36 & two-tuneA-rau-028-01+01 & - & -1 & -3 & 10130 & $\mathrm{su}$ & 0.76 & - & 0 & 0 & 0 & 0 & 0 & - \\
\hline 37 & two-tuneA-rau-028-02+01 & - & -1 & -3 & 10090 & $\mathrm{msu}$ & 1.00 & - & 0 & 0 & 0 & 0 & 0 & - \\
\hline \multirow[t]{2}{*}{38} & two-tuneA-rau-029-01+01 & - & 1 & -3 & 5790 & $\mathrm{su}$ & 0.46 & - & 0 & 0 & 0 & 0 & 0 & - \\
\hline & two-AM-rau-029-01+01 & - & 1 & -3 & 5790 & $\mathrm{su}$ & - & - & 0 & 0 & 0 & 0 & 0 & LowN,ScatRF \\
\hline 39 & two-tuneA-rau-029-02+01 & - & 1 & -3 & 9080 & $\mathrm{msu}$ & - & - & 0 & 0 & 0 & 0 & 0 & nDir \\
\hline 40 & two-tuneA-rau-030-01+01 & - & 0 & -2 & 7940 & $\mathrm{su}$ & - & - & 0 & 0 & 0 & 0 & 0 & nDir, LowN \\
\hline 41 & two-tuneA-rau-031-01+01 & - & 0 & -3 & 8790 & $\mathrm{msu}$ & - & - & 0 & 0 & 0 & 0 & 0 & nDir \\
\hline 42 & two-tuneA-rau-031-02+01 & - & 0 & -3 & 8850 & $\mathrm{msu}$ & 0.70 & - & 0 & 0 & 0 & 0 & 0 & - \\
\hline 43 & two-tuneA-rau-032-01+01 & $\mathrm{MT}^{*}$ & -2 & 2 & 8100 & $\mathrm{msu}$ & - & - & 0 & 0 & 0 & 0 & 0 & MU \\
\hline 44 & two-tuneA-rau-033-01+01 & $\mathrm{MT}^{*}$ & -2 & 0 & 8400 & $\mathrm{msu}$ & 0.74 & - & 0 & 0 & 0 & 0 & 0 & MU, EccRF \\
\hline 45 & two-tuneA-rau-033-02+01 & $\mathrm{MT}^{*}$ & -2 & 0 & 8460 & $\mathrm{su}$ & 0.66 & - & 0 & 0 & 0 & 0 & 0 & EccRF \\
\hline 46 & two-tuneA-rau-033-03+01 & $\mathrm{MT}^{*}$ & -2 & 0 & 8130 & $\mathrm{su}$ & - & - & 0 & 0 & 0 & 0 & 0 & EccRF \\
\hline 47 & two-tuneA-rau-034-01+01 & - & -2 & -3 & 10420 & $\mathrm{su}$ & - & - & 0 & 0 & 0 & 0 & 0 & LowN \\
\hline 48 & two-tuneA-rau-034-02+01 & - & -2 & -3 & 10870 & $\mathrm{su}$ & - & - & 0 & 0 & 0 & 0 & 0 & LowN \\
\hline 49 & two-tuneA-rau-035-01+01 & - & -1 & 0 & 7450 & $\mathrm{msu}$ & - & - & 0 & 0 & 0 & 0 & 0 & MU, LowN \\
\hline
\end{tabular}




\begin{tabular}{|c|c|c|c|c|c|c|c|c|c|c|c|c|c|c|}
\hline 50 & two-tuneA-rau-036-01+01 & - & -3 & 0 & 5050 & $\mathrm{su}$ & - & - & 0 & 0 & 0 & 0 & 0 & nDir \\
\hline 51 & two-tuneA-rau-036-02+01 & - & -3 & 0 & 5150 & $\mathrm{msu}$ & - & - & 0 & 0 & 0 & 0 & 0 & MU \\
\hline 52 & two-tuneA-rau-37-01+01 & MT & -1 & 3 & 3930 & $\mathrm{su}$ & - & - & 0 & 0 & 0 & 0 & 0 & nDir \\
\hline \multirow[t]{2}{*}{53} & two-tuneA-rau-37-02+01 & MT & -1 & 3 & 3400 & $\mathrm{mu}$ & - & - & 0 & 0 & 0 & 0 & 0 & MU \\
\hline & two-tuneA-rau-37-02+01 & MT & -1 & 3 & 3380 & $\mathrm{su}$ & - & - & 0 & 0 & 0 & 0 & 0 & - \\
\hline \multirow[t]{2}{*}{54} & two-tuneA-rau-038-01+01 & - & -1 & -2 & 11300 & $\mathrm{msu}$ & 0.85 & - & 0 & 0 & 0 & 0 & 0 & - \\
\hline & two-AM-rau-038-01+01 & - & -1 & -2 & 11300 & $\mathrm{msu}$ & - & - & 0 & 0 & 0 & 0 & 0 & MU, LowN \\
\hline \multirow[t]{3}{*}{55} & two-AM-rau-038-02+01 & - & -1 & -2 & 11300 & $\mathrm{msu}$ & - & - & 0 & 0 & 0 & 0 & 0 & MU, ScatRF \\
\hline & two-AM-rau-038-02+02 & - & -1 & -2 & 11300 & $\mathrm{msu}$ & - & - & 0 & 0 & 0 & 0 & 0 & MU, ScatRF \\
\hline & two-tuneA-rau-038-02+01 & - & -1 & -2 & 11300 & $\mathrm{msu}$ & - & - & 0 & 0 & 0 & 0 & 0 & MU \\
\hline 56 & two-tuneA-rau-039-01+01 & - & -2 & -3 & 9210 & $\mathrm{su}$ & - & - & 0 & 0 & 0 & 0 & 0 & nDir \\
\hline 57 & two-tuneA-rau-039-02+01 & - & -2 & -3 & 10340 & $\mathrm{su}$ & - & - & 0 & 0 & 0 & 0 & 0 & nDir \\
\hline \multirow[t]{3}{*}{58} & two-tuneA-rau-040-01+01 & MT & -1 & 2 & 7690 & $\mathrm{msu}$ & - & - & 0 & 0 & 0 & 0 & 0 & MU \\
\hline & two-tuneA-rau-040-01+02 & MT & -1 & 2 & 7920 & $\mathrm{msu}$ & - & - & 0 & 0 & 0 & 0 & 0 & MU \\
\hline & two-tuneA-rau-040-01+03 & MT & -1 & 2 & 7940 & $\mathrm{msu}$ & - & - & 0 & 0 & 0 & 0 & 0 & ok \\
\hline \multirow[t]{4}{*}{59} & two-AM-rau-040-04+01 & MT & -1 & 2 & 7940 & $\mathrm{su}$ & 0.92 & 9.2 & 1 & 1 & 1 & 1 & 1 & ok \\
\hline & two-AM-rau-040-04+02 & MT & -1 & 2 & 7940 & $\mathrm{su}$ & - & 9.2 & 0 & 0 & 0 & 0 & 0 & ok \\
\hline & two-tuneA-rau-040-04+01 & MT & -1 & 2 & 7940 & $\mathrm{su}$ & - & - & 0 & 0 & 0 & 0 & 0 & ok \\
\hline & two-AM-rau-040-04+03 & MT & -1 & 2 & 7940 & $\mathrm{su}$ & - & - & 0 & 0 & 0 & 0 & 0 & LowN \\
\hline 60 & two-tuneA-rau-041-01+01 & - & 0 & 2 & 2510 & $\mathrm{su}$ & - & - & 0 & 0 & 0 & 0 & 0 & nDir \\
\hline 61 & two-tuneA-rau-041-02+01 & - & 0 & 2 & 2580 & $\mathrm{msu}$ & 0.63 & - & 0 & 0 & 0 & 0 & 0 & nDir \\
\hline \multirow[t]{2}{*}{62} & two-tuneA-rau-042-01+01 & - & -2 & 1 & 6680 & $\mathrm{msu}$ & - & - & 0 & 0 & 0 & 0 & 0 & nDir \\
\hline & two-tuneA-rau-042-01+02 & - & -2 & 1 & 6680 & $\mathrm{msu}$ & - & - & 0 & 0 & 0 & 0 & 0 & nDir \\
\hline 63 & two-tuneA-rau-043-01+01 & - & -1 & -2 & 8710 & $\mathrm{msu}$ & - & - & 0 & 0 & 0 & 0 & 0 & MU \\
\hline 64 & two-tuneA-rau-043-02+01 & - & -1 & -2 & 8750 & $\mathrm{msu}$ & - & - & 0 & 0 & 0 & 0 & 0 & - \\
\hline 65 & two-tuneA-rau-043-02+02 & - & -1 & -2 & 8750 & $\mathrm{msu}$ & - & - & 0 & 0 & 0 & 0 & 0 & - \\
\hline 66 & two-tuneA-rau-043-03+01 & - & -1 & -2 & 8520 & $\mathrm{msu}$ & - & - & 0 & 0 & 0 & 0 & 0 & noResp \\
\hline
\end{tabular}


Table B.1 continued

cell dataFile

area $\mathrm{x} \quad \mathrm{y}$ depth isol. DI $\mathrm{n} \quad \operatorname{dir} \quad \mathrm{RF}$ slice vect. RF note on exclusion tun. gain shift shift size

\begin{tabular}{|c|c|c|c|c|c|c|c|c|c|c|c|c|c|c|}
\hline & & & & & & & & & & & & & & \\
\hline \multirow[t]{2}{*}{67} & two-tuneA-rau-043-04+01 & - & -1 & -2 & 8380 & $\mathrm{msu}$ & - & - & 0 & 0 & 0 & 0 & 0 & ok \\
\hline & two-AM-rau-043-04+01 & - & -1 & -2 & 8380 & $\mathrm{msu}$ & 0.70 & 10.4 & 1 & 1 & 1 & 1 & 1 & ok \\
\hline \multirow[t]{3}{*}{68} & two-tuneA-rau-044-01+01 & - & -2 & -4 & 6750 & $\mathrm{su}$ & 0.71 & - & 0 & 0 & 0 & 0 & 0 & ok \\
\hline & two-AM-rau-044-01+01 & - & -2 & -4 & 6750 & $\mathrm{su}$ & 0.37 & 13.1 & 1 & 1 & 0 & 1 & 1 & ok \\
\hline & two-AM-rau-044-01+02 & - & -2 & -4 & 6750 & $\mathrm{su}$ & 0.40 & - & 0 & 0 & 0 & 0 & 0 & noExp \\
\hline \multirow[t]{2}{*}{69} & two-tuneA-rau-045-01+01 & $\mathrm{MST}^{*}$ & -1 & 4 & 4900 & msu & - & - & 0 & 0 & 0 & 0 & 0 & MU \\
\hline & two-tuneA-rau-045-01+02 & - & -1 & 4 & 4900 & msu & - & - & 0 & 0 & 0 & 0 & 0 & MU \\
\hline 70 & two-tuneA-rau-045-02+01 & - & -1 & 4 & 5060 & $\mathrm{su}$ & - & - & 0 & 0 & 0 & 0 & 0 & nDir \\
\hline 71 & two-tuneA-rau-046-01+01 & - & -2 & -2 & 7814 & msu & 0.54 & - & 0 & 0 & 0 & 0 & 0 & MU \\
\hline 72 & two-tuneA-rau-046-02+01 & - & -2 & -2 & 7520 & msu & 0.61 & - & 0 & 0 & 0 & 0 & 0 & $\mathrm{nDir}$ \\
\hline \multirow[t]{2}{*}{73} & two-tuneA-rau-047-01+01 & - & 1 & 1 & 8330 & $\mathrm{msu}$ & 0.92 & - & 0 & 0 & 0 & 0 & 0 & - \\
\hline & two-AM-rau-047-01+01 & - & 1 & 1 & 8330 & msu & 0.94 & 9.5 & 1 & 1 & 1 & 1 & 1 & ok \\
\hline 74 & two-tuneA-rau-048-01+01 & - & 1 & 2 & 6690 & $\mathrm{su}$ & 1.00 & - & 0 & 0 & 0 & 0 & 0 & noResp \\
\hline 75 & two-tuneA-rau-049-01+01 & - & 1 & 0 & 7050 & $\mathrm{su}$ & - & - & 0 & 0 & 0 & 0 & 0 & noResp \\
\hline 76 & two-tuneA-rau-049-02+01 & - & 1 & 0 & 6955 & $\mathrm{msu}$ & 1.00 & - & 0 & 0 & 0 & 0 & 0 & LowN \\
\hline \multirow[t]{2}{*}{77} & two-tuneA-rau-050-01+01 & MT & -1 & 2 & 8710 & msu & - & - & 0 & 0 & 0 & 0 & 0 & MU \\
\hline & two-AM-rau-050-01+01 & MT & -1 & 2 & 8710 & msu & 0.80 & - & 0 & 0 & 0 & 0 & 0 & MU, ScatRF \\
\hline 78 & two-tuneA-rau-051-01+01 & MT & -1 & 3 & 7810 & msu & - & - & 0 & 0 & 0 & 0 & 0 & EccRF \\
\hline 79 & two-tuneA-rau-052-01+01 & $\mathrm{MT}^{*}$ & -2 & 1 & 5920 & $\mathrm{mu}$ & - & - & 0 & 0 & 0 & 0 & 0 & MU, EccRF \\
\hline 80 & two-tuneA-rau-052-02+01 & $\mathrm{MT}^{*}$ & -2 & 1 & 6210 & $\mathrm{mu}$ & - & - & 0 & 0 & 0 & 0 & 0 & $\mathrm{MU}$ \\
\hline 81 & two-tuneA-rau-052-03+01 & $\mathrm{MT}^{*}$ & -2 & 1 & 6300 & $\mathrm{su}$ & - & - & 0 & 0 & 0 & 0 & 0 & EccRF \\
\hline 82 & two-tuneA-rau-052-04+01 & $\mathrm{MT}^{*}$ & -2 & 1 & 7200 & $\mathrm{su}$ & 0.73 & - & 0 & 0 & 0 & 0 & 0 & MU, nDir \\
\hline 83 & two-tuneA-rau-053-01+01 & - & -2 & -1 & 2560 & $\mathrm{su}$ & 0.68 & - & 0 & 0 & 0 & 0 & 0 & LowN \\
\hline 84 & two-tuneA-rau-054-01+01 & $\mathrm{MT}^{*}$ & -2 & 1 & 7940 & msu & - & - & 0 & 0 & 0 & 0 & 0 & MU \\
\hline 85 & two-tuneA-rau-054-02+01 & $\mathrm{MT}^{*}$ & -2 & 1 & 8420 & msu & - & - & 0 & 0 & 0 & 0 & 0 & MU \\
\hline 86 & two-AM-rau-054-03+01 & $\mathrm{MT}^{*}$ & -2 & 1 & 8420 & msu & 0.96 & - & 0 & 0 & 0 & 0 & 0 & MU, LowN \\
\hline 87 & two-AM-rau-054-04+01 & $\mathrm{MT}^{*}$ & -2 & 1 & 8420 & msu & 0.92 & 12.7 & 1 & 1 & 1 & 1 & 1 & ok \\
\hline
\end{tabular}


.. $\quad$ Table B.1 continued

\begin{tabular}{|c|c|c|c|c|c|c|c|c|c|c|c|c|c|c|}
\hline cell & ataFile & area & $\mathrm{x}$ & $\mathrm{y}$ & depth & isol. & DI & $\mathrm{n}$ & $\begin{array}{l}\text { dir } \\
\text { tun. }\end{array}$ & $\begin{array}{l}\mathrm{RF} \\
\text { gain }\end{array}$ & $\begin{array}{l}\text { slice } \\
\text { shift }\end{array}$ & $\begin{array}{l}\text { vect. } \\
\text { shift }\end{array}$ & $\begin{array}{l}\mathrm{RF} \\
\text { size }\end{array}$ & note on exclusion \\
\hline \multirow[t]{2}{*}{88} & two-AM-rau-055-01+01 & $\mathrm{MT}$ & -1 & 1 & 6880 & $\mathrm{su}$ & 0.92 & 10.6 & 1 & 1 & 1 & 1 & 1 & ok \\
\hline & two-tuneA-rau-055-01+01 & $\mathrm{MT}$ & -1 & 1 & 6880 & $\mathrm{su}$ & - & - & 0 & 0 & 0 & 0 & 0 & ok \\
\hline 89 & two-tuneA-rau-056-01+01 & $\mathrm{MT}$ & -1 & 1 & 8550 & msu & 1.00 & - & 0 & 0 & 0 & 0 & 0 & MU \\
\hline 90 & two-tuneA-rau-056-02+01 & $\mathrm{MT}$ & -1 & 1 & 8500 & msu & - & - & 0 & 0 & 0 & 0 & 0 & LowN \\
\hline 91 & two-tuneA-rau-057-01+01 & $\mathrm{MT}^{*}$ & -3 & 2 & 8480 & msu & - & - & 0 & 0 & 0 & 0 & 0 & MU, EccRF \\
\hline \multirow[t]{2}{*}{92} & two-tuneA-rau-057-02+01 & $\mathrm{MT}^{*}$ & -3 & 2 & 8550 & $\mathrm{msu}$ & - & - & 0 & 0 & 0 & 0 & 0 & ok \\
\hline & two-AM-rau-057-02+01 & $\mathrm{MT}^{*}$ & -3 & 2 & 8550 & $\mathrm{msu}$ & 0.88 & 5.5 & 1 & 1 & 1 & 1 & 1 & ok \\
\hline 93 & two-tuneA-rau-058-01+01 & - & -1 & -2 & 11610 & $\mathrm{su}$ & - & - & 0 & 0 & 0 & 0 & 0 & LowN, nDir \\
\hline 94 & two-tuneA-rau-058-02+01 & - & -1 & -2 & 11340 & $\mathrm{su}$ & - & - & 0 & 0 & 0 & 0 & 0 & $\mathrm{nDir}$ \\
\hline 95 & two-tuneA-rau-059-01+01 & MT & -1 & 2 & 11010 & msu & 0.88 & - & 0 & 0 & 0 & 0 & 0 & LowN \\
\hline 96 & two-tuneA-rau-060-01+01 & $\mathrm{MT}^{*}$ & -2 & 1 & 8150 & msu & - & - & 0 & 0 & 0 & 0 & 0 & MU \\
\hline \multirow[t]{2}{*}{97} & two-tuneA-rau-060-02+01 & $\mathrm{MT}^{*}$ & -2 & 1 & 8980 & $\mathrm{su}$ & - & - & 0 & 0 & 0 & 0 & 0 & ok \\
\hline & two-AM-rau-060-02+01 & $\mathrm{MT}^{*}$ & -2 & 1 & 8980 & $\mathrm{su}$ & 0.98 & 6.4 & 1 & 1 & 1 & 1 & 1 & ok \\
\hline \multirow[t]{2}{*}{98} & two-tuneA-rau-061-01+01 & $\mathrm{MT}$ & -1 & 1 & 7720 & $\mathrm{msu}$ & 0.78 & - & 0 & 0 & 0 & 0 & 0 & ok \\
\hline & two-AM-rau-061-01+01 & MT & -1 & 1 & 7720 & $\mathrm{msu}$ & 0.86 & 14.1 & 1 & 1 & 1 & 1 & 1 & ok \\
\hline 99 & two-tuneA-rau-062-01+01 & MT & -1 & 1 & 6480 & $\mathrm{mu}$ & - & - & 0 & 0 & 0 & 0 & 0 & MU \\
\hline 100 & two-tuneA-rau-062-02+01 & MT & -1 & 1 & 6180 & $\mathrm{msu}$ & - & - & 0 & 0 & 0 & 0 & 0 & $\mathrm{MU}$ \\
\hline \multirow[t]{2}{*}{101} & two-tuneA-rau-062-03+01 & MT & -1 & 1 & 6510 & $\mathrm{su}$ & 0.71 & - & 0 & 0 & 0 & 0 & 0 & ok \\
\hline & two-AM-rau-062-03+01 & $\mathrm{MT}$ & -1 & 1 & 6510 & $\mathrm{su}$ & 0.68 & 6.7 & 1 & 1 & 1 & 1 & 1 & ok \\
\hline 102 & two-tuneA-rau-063-01+01 & $\mathrm{MT}$ & -0.5 & 0 & 5500 & $\mathrm{mu}$ & 0.71 & - & 0 & 0 & 0 & 0 & 0 & MU \\
\hline \multirow[t]{2}{*}{103} & two-tuneA-rau-063-02+01 & $\mathrm{MT}$ & -0.5 & 0 & 5540 & $\mathrm{msu}$ & 0.92 & - & 0 & 0 & 0 & 0 & 0 & MU \\
\hline & two-AM-rau-063-02+01 & MT & -0.5 & 0 & 5540 & msu & 0.65 & - & 0 & 0 & 0 & 0 & 0 & MU, LowN \\
\hline 104 & two-tuneA-rau-064-01+01 & MT & -1.5 & 0 & 5530 & msu & 0.73 & - & 0 & 0 & 0 & 0 & 0 & MU, LowN \\
\hline \multirow[t]{2}{*}{105} & two-tuneA-rau-064-02+01 & $\mathrm{MT}^{*}$ & -1.5 & 0 & 6080 & msu & - & - & 0 & 0 & 0 & 0 & 0 & LowN \\
\hline & two-AM-rau-064-02+01 & $\mathrm{MT}^{*}$ & -1.5 & 0 & 6080 & msu & - & - & 0 & 0 & 0 & 0 & 0 & LowN \\
\hline \multirow[t]{2}{*}{106} & two-tuneA-rau-065-01+01 & MT & -1 & 1 & 7690 & $\mathrm{su}$ & 1.00 & - & 0 & 0 & 0 & 0 & 0 & ok \\
\hline & two-AM-rau-065-01+01 & MT & -1 & 1 & 7690 & $\mathrm{su}$ & 0.96 & 10.3 & 1 & 1 & 1 & 1 & 1 & ok \\
\hline \multirow[t]{2}{*}{107} & two-tuneA-rau-066-01+01 & $\mathrm{MT}$ & -1 & 2 & 7390 & msu & - & - & 0 & 0 & 0 & 0 & 0 & \\
\hline & two-AM-rau-066-01+01 & MT & -1 & 2 & 7390 & msu & 0.93 & 10.5 & 1 & 1 & 1 & 1 & 0 & ok \\
\hline 108 & two-tuneA-rau-066-02+01 & MT & -1 & 2 & 7840 & $\mathrm{su}$ & - & - & 0 & 0 & 0 & 0 & 0 & LowN \\
\hline
\end{tabular}


... Table B.1 continued cell dataFile

area $\mathrm{x} \quad \mathrm{y}$ depth isol. DI $\mathrm{n}$ dir $\mathrm{RF}$ slice vect. RF note on exclusion

\begin{tabular}{|c|c|c|c|c|c|c|c|c|c|c|c|c|c|c|}
\hline & & & & & & & & & & & & & & \\
\hline \multirow[t]{2}{*}{109} & two-tuneA-rau-067-01+01 & $\mathrm{MT}$ & -1 & 1 & 6540 & $\mathrm{msu}$ & - & - & 0 & 0 & 0 & 0 & 0 & ok \\
\hline & two-AM-rau-067-01+01 & MT & -1 & 1 & 6540 & $\mathrm{msu}$ & 0.77 & 8.2 & 1 & 1 & 1 & 1 & 1 & ok \\
\hline 110 & two-tuneA-rau-067-01+02 & $\mathrm{MT}$ & -1 & 1 & 6540 & $\mathrm{msu}$ & - & - & 0 & 0 & 0 & 0 & 0 & LowN \\
\hline \multirow[t]{2}{*}{111} & two-tuneA-rau-068-01+01 & MT & -1 & 1 & 8420 & $\mathrm{msu}$ & - & - & 0 & 0 & 0 & 0 & 0 & LowN \\
\hline & two-AM-rau-068-01+01 & MT & -1 & 1 & 8420 & $\mathrm{msu}$ & - & - & 0 & 0 & 0 & 0 & 0 & LowN \\
\hline 112 & two-AM-rau-068-02+01 & MT & -1 & 1 & 8450 & $\mathrm{su}$ & 0.89 & 7.4 & 1 & 1 & 1 & 1 & 1 & ok \\
\hline 113 & two-tuneA-rau-069-01+01 & MT & -1 & 1 & 7060 & $\mathrm{su}$ & 1.00 & - & 0 & 0 & 0 & 0 & 0 & EccRF \\
\hline 114 & two-tuneA-rau-069-02+01 & MT & -1 & 1 & 7900 & $\mathrm{msu}$ & - & - & 0 & 0 & 0 & 0 & 0 & MU \\
\hline 115 & two-tuneA-rau-069-03+01 & MT & -1 & 1 & 6450 & $\mathrm{msu}$ & - & - & 0 & 0 & 0 & 0 & 0 & ScatRF \\
\hline \multirow[t]{2}{*}{116} & two-AM-rau-069-03+01 & MT & -1 & 1 & 6450 & $\mathrm{msu}$ & 0.52 & - & 0 & 0 & 0 & 0 & 0 & ScatRF \\
\hline & two-tuneA-rau-069-03+02 & MT & -1 & 1 & 6450 & $\mathrm{msu}$ & - & - & 0 & 0 & 0 & 0 & 0 & LowN \\
\hline 117 & two-tuneA-rau-070-01+02 & $\mathrm{MT}$ & -1 & 1 & 6490 & $\mathrm{su}$ & - & - & 0 & 0 & 0 & 0 & 0 & $\mathrm{nDir}$ \\
\hline 118 & two-tuneA-rau-070-02+01 & MT & -1 & 1 & 8840 & $\mathrm{su}$ & - & - & 0 & 0 & 0 & 0 & 0 & MU \\
\hline 119 & two-tuneA-rau-071-01+01 & - & 1 & 0 & 10750 & $\mathrm{msu}$ & 0.72 & - & 0 & 0 & 0 & 0 & 0 & ok \\
\hline 120 & two-AM-rau-071-02+01 & - & 1 & 0 & 10860 & su & 0.45 & 6.6 & 1 & 1 & 1 & 1 & 1 & ok \\
\hline \multirow[t]{2}{*}{121} & two-tuneA-rau-072-01+01 & $\mathrm{MT}^{*}$ & -2 & 0 & 8160 & $\mathrm{msu}$ & - & - & 0 & 0 & 0 & 0 & 0 & nDir \\
\hline & two-AM-rau-072-01+01 & $\mathrm{MT}^{*}$ & -2 & 0 & 8160 & $\mathrm{msu}$ & 0.54 & 13 & 1 & 1 & 1 & 1 & 1 & ok \\
\hline \multirow[t]{2}{*}{122} & two-tuneA-rau-073-01+01 & MT & -1 & 1 & 8860 & $\mathrm{msu}$ & - & - & 0 & 0 & 0 & 0 & 0 & LowN \\
\hline & two-AM-rau-073-01+01 & MT & -1 & 1 & 8860 & $\mathrm{msu}$ & 0.47 & - & 0 & 0 & 0 & 0 & 0 & ScatRF \\
\hline 123 & two-tuneA-rau-074-01+01 & $\mathrm{MT}^{*}$ & -1 & 0 & 6510 & $\mathrm{msu}$ & - & - & 0 & 0 & 0 & 0 & 0 & $\mathrm{MU}$ \\
\hline \multirow[t]{3}{*}{124} & two-tuneA-rau-075-01+01 & MT & -1 & 2 & 5900 & $\mathrm{msu}$ & - & - & 0 & 0 & 0 & 0 & 0 & ok \\
\hline & two-AM-rau-075-01+01 & MT & -1 & 2 & 5900 & $\mathrm{msu}$ & 0.91 & 7.2 & 1 & 1 & 1 & 0 & 1 & ok \\
\hline & two-AM-rau-075-01+02 & $\mathrm{MT}$ & -1 & 2 & 5900 & $\mathrm{msu}$ & - & - & 0 & 0 & 0 & 0 & 0 & noExp,LowN \\
\hline \multirow[t]{2}{*}{125} & two-tuneA-rau-076-01+01 & MT & -1 & 1 & 6450 & $\mathrm{msu}$ & - & - & 0 & 0 & 0 & 0 & 0 & MU \\
\hline & two-AM-rau-076-01+01 & MT & -1 & 1 & 6450 & $\mathrm{msu}$ & - & - & 0 & 0 & 0 & 0 & 0 & MU \\
\hline 126 & two-AM-rau-076-02+01 & MT & -1 & 1 & 6450 & $\mathrm{msu}$ & - & 14.4 & 1 & 1 & 1 & 0 & 1 & ok \\
\hline \multirow[t]{3}{*}{127} & two-tuneA-rau-077-01+01 & $\mathrm{MT}^{*}$ & -2 & 1 & 7510 & $\mathrm{msu}$ & - & - & 0 & 0 & 0 & 0 & 0 & MU \\
\hline & two-tuneA-rau-077-01+02 & $\mathrm{MT}^{*}$ & -2 & 1 & 7510 & $\mathrm{msu}$ & - & - & 0 & 0 & 0 & 0 & 0 & ok \\
\hline & two-AM-rau-077-01+02 & $\mathrm{MT}^{*}$ & -2 & 1 & 7510 & $\mathrm{msu}$ & 0.76 & 15.1 & 1 & 1 & 1 & 1 & 1 & ok \\
\hline
\end{tabular}


... Table B.1 continued

cell dataFile

area $x \quad y$ depth isol. DI $n$ dir $R F$ slice vect. RF note on exclusion

\begin{tabular}{|c|c|c|c|c|c|c|c|c|c|c|c|c|c|c|}
\hline 128 & two-tuneA-rau-078-01+01 & - & -1 & 1 & 5670 & $\mathrm{msu}$ & - & - & 0 & 0 & 0 & 0 & 0 & nDir \\
\hline 129 & two-tuneA-rau-079-01+01 & $\mathrm{MT}^{*}$ & -2 & 1 & 7080 & $\mathrm{msu}$ & 0.89 & - & 0 & 0 & 0 & 0 & 0 & nDir \\
\hline \multirow[t]{2}{*}{130} & two-tuneA-rau-079-02+01 & $\mathrm{MT}^{*}$ & -2 & 1 & 7280 & $\mathrm{msu}$ & 0.89 & - & 0 & 0 & 0 & 0 & 0 & ok \\
\hline & two-AM-rau-079-02+01 & MT* & -2 & 1 & 7280 & $\mathrm{msu}$ & 0.89 & 7.4 & 1 & 1 & 1 & 1 & 1 & ok \\
\hline \multirow[t]{2}{*}{131} & two-tuneA-rau-080-01+01 & MT & -1 & 1 & 6250 & $\mathrm{su}$ & - & - & 0 & 0 & 0 & 0 & 0 & LowN \\
\hline & two-AM-rau-080-01+01 & MT & -1 & 1 & 6250 & $\mathrm{su}$ & - & - & 0 & 0 & 0 & 0 & 0 & LowN \\
\hline \multirow[t]{2}{*}{132} & two-tuneA-rau-080-02+01 & MT & -1 & 1 & 6500 & $\mathrm{mu}$ & - & - & 0 & 0 & 0 & 0 & 0 & MU \\
\hline & two-AM-rau-080-02+01 & MT & -1 & 1 & 6500 & $\mathrm{mu}$ & - & - & 0 & 0 & 0 & 0 & 0 & MU \\
\hline 133 & two-tuneA-rau-080-03+01 & MT & -1 & 1 & 6400 & $\mathrm{su}$ & - & - & 0 & 0 & 0 & 0 & 0 & LowN \\
\hline 134 & two-tuneA-rau-081-01+01 & MT & -1 & 1 & 7010 & $\mathrm{msu}$ & 1.00 & - & 0 & 0 & 0 & 0 & 0 & MU \\
\hline \multirow[t]{3}{*}{135} & two-AM-rau-081-02+01 & MT & -1 & 1 & 6980 & $\mathrm{msu}$ & 0.79 & 18.7 & 1 & 1 & 1 & 1 & 1 & ok \\
\hline & two-tuneA-rau-081-02+01 & MT & -1 & 1 & 6980 & $\mathrm{msu}$ & - & - & 0 & 0 & 0 & 0 & 0 & ok \\
\hline & two-tuneA-rau-081-02+02 & MT & -1 & 1 & 6980 & $\mathrm{msu}$ & 0.93 & - & 0 & 0 & 0 & 0 & 0 & noExp \\
\hline \multirow[t]{3}{*}{136} & two-tuneA-rau-082-01+01 & MT & -1 & 1 & 5250 & $\mathrm{msu}$ & - & - & 0 & 0 & 0 & 0 & 0 & ok \\
\hline & two-AM-rau-082-01+01 & MT & -1 & 1 & 5250 & $\mathrm{msu}$ & 0.95 & 13.3 & 1 & 1 & 1 & 1 & 1 & ok \\
\hline & two-AM-rau-082-01+02 & MT & -1 & 1 & 5250 & $\mathrm{msu}$ & 0.90 & - & 0 & 0 & 0 & 0 & 0 & noExp \\
\hline \multirow[t]{4}{*}{137} & two-tuneA-rau-083-01+01 & MT & -1 & 1 & 6700 & $\mathrm{msu}$ & - & - & 0 & 0 & 0 & 0 & 0 & ok \\
\hline & two-AM-rau-083-01+01 & MT & -1 & 1 & 6700 & $\mathrm{msu}$ & 0.89 & 15.2 & 1 & 1 & 1 & 1 & 1 & ok \\
\hline & two-AM-rau-083-01+02 & MT & -1 & 1 & 6700 & $\mathrm{msu}$ & 0.87 & - & 0 & 0 & 0 & 0 & 0 & LowN \\
\hline & two-AM-rau-083-01+03 & MT & -1 & 1 & 6700 & $\mathrm{msu}$ & 0.91 & - & 0 & 0 & 0 & 0 & 0 & noExp \\
\hline \multirow[t]{3}{*}{138} & two-tuneA-rau-084-01+01 & MT & -1 & 2 & 7630 & $\mathrm{su}$ & - & - & 0 & 0 & 0 & 0 & 0 & LowN \\
\hline & two-AM-rau-084-01+01 & MT & -1 & 2 & 7630 & $\mathrm{su}$ & 0.97 & 11.8 & 1 & 1 & 1 & 1 & 1 & ok \\
\hline & two-AM-rau-084-01+02 & MT & -1 & 2 & 7630 & $\mathrm{su}$ & 1.00 & - & 0 & 0 & 0 & 0 & 0 & noExp \\
\hline \multirow[t]{3}{*}{139} & two-tuneA-rau-085-01+01 & MT & -1 & 2 & 8050 & $\mathrm{su}$ & - & - & 0 & 0 & 0 & 0 & 0 & ok \\
\hline & two-AM-rau-085-01+01 & MT & -1 & 2 & 8050 & $\mathrm{su}$ & 0.95 & 18.2 & 1 & 1 & 1 & 0 & 1 & ok \\
\hline & two-AM-rau-085-01+02 & MT & -1 & 2 & 8050 & $\mathrm{su}$ & 1.00 & - & 0 & 0 & 0 & 0 & 0 & noExp \\
\hline
\end{tabular}


... Table B.1 continued

area $\mathrm{x} \quad \mathrm{y}$ depth isol. DI $\mathrm{n}$ dir $\mathrm{RF}$ slice vect. RF note on exclusion

tun. gain shift shift size

\begin{tabular}{|c|c|c|c|c|c|c|c|c|c|c|c|c|c|c|}
\hline \multirow[t]{2}{*}{140} & two-tuneA-rau-086-01+01 & MT & -1 & 1 & 5590 & $\mathrm{mu}$ & - & - & 0 & 0 & 0 & 0 & 0 & lowN \\
\hline & two-AM-rau-086-01+01 & MT & -1 & 1 & 5590 & $\mathrm{mu}$ & - & - & 0 & 0 & 0 & 0 & 0 & MU \\
\hline \multirow[t]{2}{*}{141} & two-tuneA-rau-087-01+01 & MT & -1 & 1 & 7370 & $\mathrm{mu}$ & - & - & 0 & 0 & 0 & 0 & 0 & MU \\
\hline & two-AM-rau-087-01+01 & MT & -1 & 1 & 7370 & $\mathrm{mu}$ & - & - & 0 & 0 & 0 & 0 & 0 & MU, noExp \\
\hline \multirow[t]{2}{*}{142} & two-tuneA-rau-087-02+01 & MT & -1 & 1 & 7580 & $\mathrm{su}$ & - & - & 0 & 0 & 0 & 0 & 0 & ok \\
\hline & two-AM-rau-087-02+01 & $\mathrm{MT}$ & -1 & 1 & 7580 & $\mathrm{su}$ & 0.80 & 14.5 & 1 & 1 & 1 & 1 & 1 & ok \\
\hline \multirow[t]{3}{*}{143} & two-tuneA-rau-088-01+01 & $\mathrm{MT}$ & -1 & 1 & 6775 & $\mathrm{msu}$ & - & - & 0 & 0 & 0 & 0 & 0 & ok \\
\hline & two-AM-rau-088-01+01 & $\mathrm{MT}$ & -1 & 1 & 6775 & $\mathrm{msu}$ & 0.74 & 15.8 & 1 & 1 & 1 & 1 & 1 & ok \\
\hline & two-AM-rau-088-01+02 & MT & -1 & 1 & 6775 & msu & 0.78 & - & 0 & 0 & 0 & 0 & 0 & noExp \\
\hline \multirow[t]{3}{*}{144} & two-tuneA-rau-089-01+01 & MT & -1 & 1 & 4450 & msu & - & - & 0 & 0 & 0 & 0 & 0 & ok \\
\hline & two-AM-rau-089-01+01 & MT & -1 & 1 & 4450 & $\mathrm{msu}$ & 0.76 & 19.9 & 1 & 1 & 1 & 1 & 1 & ok \\
\hline & two-AM-rau-089-01+02 & MT & -1 & 1 & 4450 & $\mathrm{msu}$ & - & - & 0 & 0 & 0 & 0 & 0 & noExp \\
\hline 145 & two-tuneA-rau-090-01+01 & MT & -1 & 1 & 7500 & $\mathrm{msu}$ & - & - & 0 & 0 & 0 & 0 & 0 & $\mathrm{Mu}$ \\
\hline \multirow[t]{3}{*}{146} & two-tuneA-rau-090-02+01 & MT & -1 & 1 & 7540 & $\mathrm{msu}$ & - & - & 0 & 0 & 0 & 0 & 0 & ok \\
\hline & two-AM-rau-090-02+01 & $\mathrm{MT}$ & -1 & 1 & 7540 & msu & - & - & 0 & 0 & 0 & 0 & 0 & LowN \\
\hline & two-AM-rau-090-02+02 & MT & -1 & 1 & 7540 & msu & 0.67 & 11.3 & 1 & 1 & 1 & 1 & 1 & ok \\
\hline 147 & two-tuneA-rau-091-01+01 & MT & -1 & 1 & 5500 & $\mathrm{su}$ & - & - & 0 & 0 & 0 & 0 & 0 & noResp \\
\hline \multirow[t]{3}{*}{148} & two-tuneA-rau-091-02+01 & MT & -1 & 1 & 5800 & $\mathrm{msu}$ & - & - & 0 & 0 & 0 & 0 & 0 & ok \\
\hline & two-AM-rau-091-02+01 & MT & -1 & 1 & 5800 & $\mathrm{msu}$ & 0.91 & 8.5 & 1 & 1 & 1 & 1 & 1 & ok \\
\hline & two-AM-rau-091-02+02 & MT & -1 & 1 & 5800 & $\mathrm{msu}$ & - & - & 0 & 0 & 0 & 0 & 0 & noExp \\
\hline \multirow[t]{2}{*}{149} & two-AM-rau-091-03+01 & MT & -1 & 1 & 5900 & $\mathrm{su}$ & - & - & 0 & 0 & 0 & 0 & 0 & noExp \\
\hline & two-AM-rau-091-03+02 & MT & -1 & 1 & 5900 & $\mathrm{su}$ & - & - & 0 & 0 & 0 & 0 & 0 & LowN \\
\hline \multirow[t]{2}{*}{150} & two-tuneA-rau-092-01+01 & MT & -1 & 1 & 3740 & $\mathrm{su}$ & - & - & 0 & 0 & 0 & 0 & 0 & ok \\
\hline & two-AM-rau-092-01+01 & MT & -1 & 1 & 3740 & $\mathrm{su}$ & 0.91 & 15.8 & 1 & 1 & 1 & 1 & 1 & ok \\
\hline 151 & two-tuneA-rau-093-01+01 & $\mathrm{MT}$ & -1 & 1 & 6100 & $\mathrm{su}$ & - & - & 0 & 0 & 0 & 0 & 0 & ok \\
\hline 152 & two-AM-rau-093-02+01 & MT & -1 & 1 & 6400 & $\mathrm{su}$ & 0.98 & 13.9 & 1 & 1 & 1 & 1 & 1 & ok \\
\hline 153 & two-tuneA-rau-094-01+01 & MT & -1 & 1 & 6280 & msu & - & - & 0 & 0 & 0 & 0 & 0 & ok \\
\hline 154 & two-AM-rau-094-01+01 & MT & -1 & 1 & 6280 & $\mathrm{msu}$ & 0.86 & 9.7 & 1 & 1 & 1 & 1 & 1 & ok \\
\hline
\end{tabular}


... Table B.1 continued

\begin{tabular}{|c|c|c|c|c|c|c|c|c|c|c|c|c|c|c|}
\hline cell & ataFile & area & $\mathrm{x}$ & $\mathrm{y}$ & depth & isol. & DI & $\mathrm{n}$ & $\begin{array}{l}\text { dir } \\
\text { tun. }\end{array}$ & $\begin{array}{l}\mathrm{RF} \\
\text { gain }\end{array}$ & $\begin{array}{l}\text { slice } \\
\text { shift }\end{array}$ & $\begin{array}{l}\text { vect. } \\
\text { shift }\end{array}$ & $\begin{array}{l}\mathrm{RF} \\
\text { size }\end{array}$ & note on exclusion \\
\hline \multirow[t]{2}{*}{155} & two-tuneA-rau-094-02+01 & MT & -1 & 1 & 6500 & $\mathrm{msu}$ & - & - & 0 & 0 & 0 & 0 & 0 & ok \\
\hline & two-AM-rau-094-02+01 & MT & -1 & 1 & 6500 & $\mathrm{msu}$ & 0.92 & 8.9 & 1 & 1 & 1 & 1 & 1 & ok \\
\hline 156 & two-tuneA-rau-095-01+01 & MT & -1 & 1 & 5560 & $\mathrm{su}$ & - & - & 0 & 0 & 0 & 0 & 0 & noResp \\
\hline \multirow[t]{2}{*}{157} & two-tuneA-rau-095-02+01 & MT & -1 & 1 & 5920 & $\mathrm{msu}$ & - & - & 0 & 0 & 0 & 0 & 0 & ok \\
\hline & two-AM-rau-095-02+01 & MT & -1 & 1 & 5920 & $\mathrm{msu}$ & 0.95 & 11.1 & 1 & 1 & 1 & 1 & 1 & ok \\
\hline \multirow[t]{2}{*}{158} & two-tuneA-rau-096-01+01 & MT & -1 & 1 & 6070 & $\mathrm{msu}$ & - & - & 0 & 0 & 0 & 0 & 0 & ok \\
\hline & two-AM-rau-096-01+01 & MT & -1 & 1 & 6070 & $\mathrm{msu}$ & - & - & 0 & 0 & 0 & 0 & 0 & LowN, EccRF \\
\hline \multirow[t]{2}{*}{159} & two-tuneA-rau-096-02+01 & MT & -1 & 1 & 6340 & $\mathrm{msu}$ & - & - & 0 & 0 & 0 & 0 & 0 & ok \\
\hline & two-AM-rau-096-02+01 & MT & -1 & 1 & 6340 & $\mathrm{msu}$ & 0.61 & 10.7 & 1 & 1 & 1 & 1 & 1 & ok \\
\hline \multirow[t]{2}{*}{160} & two-tuneA-rau-097-01+01 & MT & -1 & 1 & 6340 & msu & - & - & 0 & 0 & 0 & 0 & 0 & ok \\
\hline & two-AM-rau-097-01+01 & MT & -1 & 1 & 6340 & $\mathrm{msu}$ & 0.66 & 13.8 & 1 & 1 & 1 & 1 & 1 & ok \\
\hline \multirow[t]{2}{*}{161} & two-tuneA-rau-097-02+01 & $\mathrm{MT}$ & -1 & 1 & 5790 & $\mathrm{msu}$ & - & - & 0 & 0 & 0 & 0 & 0 & EccRF \\
\hline & two-AM-rau-097-02+01 & $\mathrm{MT}$ & -1 & 1 & 5790 & $\mathrm{msu}$ & - & - & 0 & 0 & 0 & 0 & 0 & EccRF \\
\hline \multirow[t]{2}{*}{162} & two-tuneA-rau-098-01+01 & MT & -1 & 1 & 7900 & msu & - & - & 0 & 0 & 0 & 0 & 0 & ok \\
\hline & two-AM-rau-098-01+01 & MT & -1 & 1 & 7900 & msu & 0.84 & 13.9 & 1 & 1 & 1 & 1 & 1 & ok \\
\hline 163 & two-AM-rau-098-02+01 & MT & -1 & 1 & 8540 & $\mathrm{su}$ & 0.82 & 12.2 & 1 & 1 & 1 & 1 & 1 & ok \\
\hline 164 & two-tuneA-rau-099-01+01 & MT & -1 & 1 & 5300 & $\mathrm{msu}$ & - & - & 0 & 0 & 0 & 0 & 0 & MU \\
\hline \multirow[t]{2}{*}{165} & two-tuneA-rau-099-02+01 & MT & -1 & 1 & 4780 & $\mathrm{su}$ & - & - & 0 & 0 & 0 & 0 & 0 & ok \\
\hline & two-AM-rau-099-02+01 & MT & -1 & 1 & 4780 & $\mathrm{su}$ & 0.92 & 16.6 & 1 & 1 & 1 & 1 & 1 & ok \\
\hline \multirow[t]{2}{*}{166} & two-tuneA-rau-100-01+01 & MT & -1 & 1 & 9010 & $\mathrm{su}$ & - & - & 0 & 0 & 0 & 0 & 0 & ok \\
\hline & two-AM-rau-100-01+01 & MT & -1 & 1 & 9010 & $\mathrm{su}$ & 0.93 & 16.7 & 1 & 1 & 1 & 1 & 1 & ok \\
\hline \multirow[t]{2}{*}{167} & two-tuneA-rau-101-01+01 & MT & -1 & 1 & 7450 & $\mathrm{su}$ & - & - & 0 & 0 & 0 & 0 & 0 & ok \\
\hline & two-AM-rau-101-01+01 & MT & -1 & 1 & 7450 & $\mathrm{su}$ & 0.67 & 12 & 1 & 1 & 1 & 1 & 1 & ok \\
\hline 168 & two-AM-rau-101-02+01 & MT & -1 & 1 & 7680 & $\mathrm{su}$ & 0.86 & 10 & 1 & 1 & 1 & 1 & 1 & ok \\
\hline \multirow[t]{2}{*}{169} & two-tuneA-rau-102-01+01 & MT & -1 & 1 & 6000 & $\mathrm{msu}$ & - & - & 0 & 0 & 0 & 0 & 0 & ok \\
\hline & two-AM-rau-102-01+01 & MT & -1 & 1 & 6000 & $\mathrm{msu}$ & - & - & 0 & 0 & 0 & 0 & 0 & noRes,ScatRF \\
\hline \multirow[t]{2}{*}{170} & two-tuneA-rau-103-01+01 & MT & -1 & 1 & 8370 & $\mathrm{su}$ & - & - & 0 & 0 & 0 & 0 & 0 & ok \\
\hline & two-AM-rau-103-01+01 & MT & -1 & 1 & 8370 & $\mathrm{su}$ & 0.92 & 14.1 & 1 & 1 & 1 & 0 & 1 & ok \\
\hline
\end{tabular}


... Table B.1 continued

area $\mathrm{x} \quad \mathrm{y}$ depth isol. DI $\mathrm{n}$ dir $\mathrm{RF}$ slice vect. RF note on exclusion tun. gain shift shift size

\begin{tabular}{|c|c|c|c|c|c|c|c|c|c|c|c|c|c|c|}
\hline & & & & & & & & & & & & & & \\
\hline 171 & two-tuneA-rau-104-01+01 & MT & -1 & 0 & 5590 & $\mathrm{su}$ & - & - & 0 & 0 & 0 & 0 & 0 & ok \\
\hline & two-AM-rau-104-01+01 & $\mathrm{MT}$ & -1 & 1 & 5590 & $\mathrm{su}$ & 0.88 & 13.7 & 1 & 1 & 1 & 1 & 1 & ok \\
\hline 172 & two-tuneA-rau-105-01+01 & $\mathrm{MT}$ & -1 & 1 & 7100 & $\mathrm{msu}$ & - & - & 0 & 0 & 0 & 0 & 0 & MU \\
\hline 173 & two-AM-rau-105-02+01 & $\mathrm{MT}$ & -1 & 1 & 6960 & $\mathrm{msu}$ & 0.88 & 11.1 & 1 & 1 & 1 & 1 & 1 & ok \\
\hline 174 & two-tuneA-rau-106-01+01 & MT & -1 & 1 & 11370 & $\mathrm{su}$ & - & - & 0 & 0 & 0 & 0 & 0 & ok \\
\hline & two-AM-rau-106-01+01 & $\mathrm{MT}$ & -1 & 1 & 11370 & $\mathrm{su}$ & 0.76 & 9.7 & 1 & 1 & 1 & 0 & 1 & ok \\
\hline 175 & two-tuneA-rau-107-01+01 & MT & -1 & 1 & 7990 & $\mathrm{su}$ & - & - & 0 & 0 & 0 & 0 & 0 & ok \\
\hline & two-AM-rau-107-01+01 & MT & -1 & 1 & 7990 & $\mathrm{su}$ & 0.97 & 15.9 & 1 & 1 & 1 & 1 & 1 & ok \\
\hline 176 & two-AM-rau-108-01+01 & MT & -1 & 1 & 7970 & $\mathrm{su}$ & 0.80 & 18.6 & 1 & 1 & 1 & 0 & 1 & ok \\
\hline 177 & two-tuneA-rau-109-01+01 & MT & -1 & 1 & 7960 & $\mathrm{su}$ & - & - & 0 & 0 & 0 & 0 & 0 & noResp \\
\hline 178 & two-AM-rau-109-02+01 & MT & -1 & 1 & 8100 & $\mathrm{msu}$ & - & - & 0 & 0 & 0 & 0 & 0 & MU, LowN \\
\hline 179 & two-AM-rau-109-03+01 & MT & -1 & 1 & 7800 & $\mathrm{su}$ & 0.96 & 8.4 & 1 & 1 & 1 & 1 & 1 & ok \\
\hline 180 & two-tuneA-rau-110-01+01 & MT & -1 & 1 & 9870 & $\mathrm{msu}$ & - & - & 0 & 0 & 0 & 0 & 0 & EccRF \\
\hline & two-AM-rau-110-01+01 & $\mathrm{MT}$ & -1 & 1 & 9870 & $\mathrm{msu}$ & - & - & 0 & 0 & 0 & 0 & 0 & EccRF \\
\hline 181 & two-tuneA-rau-110-02+01 & $\mathrm{MT}$ & -1 & 1 & 10350 & $\mathrm{su}$ & - & - & 0 & 0 & 0 & 0 & 0 & ok \\
\hline & two-AM-rau-110-02+01 & $\mathrm{MT}$ & -1 & 1 & 10350 & $\mathrm{su}$ & 0.94 & 9.7 & 1 & 1 & 1 & 1 & 1 & ok \\
\hline 182 & two-tuneA-rau-111-01+01 & MT & -1 & 1 & 7290 & $\mathrm{msu}$ & - & - & 0 & 0 & 0 & 0 & 0 & ok \\
\hline & two-AM-rau-111-01+01 & $\mathrm{MT}$ & -1 & 1 & 7290 & $\mathrm{msu}$ & 0.89 & 11.4 & 0 & 1 & 1 & 1 & 1 & ok \\
\hline 183 & two-tuneA-rau-112-01+01 & $\mathrm{MT}$ & -1 & 1 & 7600 & $\mathrm{msu}$ & - & - & 0 & 0 & 0 & 0 & 0 & MU \\
\hline 184 & two-tuneA-rau-112-02+01 & $\mathrm{MT}$ & -1 & 1 & 7960 & $\mathrm{su}$ & - & - & 0 & 0 & 0 & 0 & 0 & ok \\
\hline & two-AM-rau-112-02+01 & $\mathrm{MT}$ & -1 & 1 & 7960 & $\mathrm{su}$ & 0.92 & 12.5 & 0 & 1 & 1 & 1 & 1 & $\mathrm{ok}$ \\
\hline 185 & two-tuneA-rau-112-03+01 & $\mathrm{MT}$ & -1 & 1 & 7560 & $\mathrm{mu}$ & - & - & 0 & 0 & 0 & 0 & 0 & MU \\
\hline & two-AM-rau-112-03+01 & $\mathrm{MT}$ & -1 & 1 & 7560 & $\mathrm{mu}$ & - & - & 0 & 0 & 0 & 0 & 0 & MU \\
\hline 186 & two-tuneA-rau-113-01+01 & $\mathrm{MT}$ & -2 & 1 & 7820 & $\mathrm{su}$ & - & - & 0 & 0 & 0 & 0 & 0 & ok \\
\hline & two-AM-rau-113-01+01 & $\mathrm{MT}$ & -2 & 1 & 7820 & $\mathrm{su}$ & 0.94 & 10.8 & 0 & 1 & 1 & 1 & 1 & ok \\
\hline & two-AMr-rau-113-01+01 & MT & -2 & 1 & 7820 & $\mathrm{su}$ & - & - & 0 & 0 & 0 & 0 & 0 & ok \\
\hline 187 & two-tuneA-rau-114-01+01 & MT & -2 & 1 & 8588 & $\mathrm{msu}$ & - & - & 0 & 0 & 0 & 0 & 0 & ok \\
\hline & two-AM-rau-114-01+01 & MT & -2 & 1 & 8588 & $\mathrm{msu}$ & 0.85 & 13 & 0 & 1 & 1 & 1 & 1 & ok \\
\hline
\end{tabular}


.. $\quad$ Table B.1 continued

\begin{tabular}{|c|c|c|c|c|c|c|c|c|c|c|c|c|c|c|}
\hline cell & dataFile & area & $\mathrm{x}$ & $\mathrm{y}$ & depth & isol. & DI & $\mathrm{n}$ & $\begin{array}{l}\text { dir } \\
\text { tun. }\end{array}$ & $\begin{array}{l}\mathrm{RF} \\
\text { gain }\end{array}$ & $\begin{array}{l}\text { slice } \\
\text { shift }\end{array}$ & & $\begin{array}{l}\mathrm{RF} \\
\text { size }\end{array}$ & note on exclusion \\
\hline \multirow[t]{2}{*}{188} & two-tuneA-rau-114-02+01 & MT & -2 & 1 & 7920 & $\mathrm{msu}$ & - & - & 0 & 0 & 0 & 0 & 0 & LowN \\
\hline & two-AM-rau-114-02+01 & MT & -2 & 1 & 7920 & $\mathrm{msu}$ & 0.95 & - & 0 & 0 & 0 & 0 & 0 & LowN \\
\hline 189 & two-tuneA-rau-115-01+01 & MT & -1 & 1 & 10050 & $\mathrm{su}$ & - & - & 0 & 0 & 0 & 0 & 0 & noResp \\
\hline 190 & two-AM-rau-115-02+01 & MT & -1 & 1 & 9980 & $\mathrm{msu}$ & 0.95 & - & 0 & 0 & 0 & 0 & 0 & MU, ScatRF \\
\hline 191 & two-tuneA-rau-115-03+01 & MT & -1 & 1 & 9930 & $\mathrm{su}$ & - & - & 0 & 0 & 0 & 0 & 0 & LowN \\
\hline 192 & two-tuneA-rau-116-01+01 & - & 1 & -1 & 10810 & $\mathrm{msu}$ & - & - & 0 & 0 & 0 & 0 & 0 & MU \\
\hline \multirow[t]{3}{*}{193} & two-tuneA-rau-116-02+01 & $\mathrm{MT}^{*}$ & 1 & -1 & 11100 & $\mathrm{msu}$ & - & - & 0 & 0 & 0 & 0 & 0 & ok \\
\hline & two-AM-rau-116-02+01 & $\mathrm{MT}^{*}$ & 1 & -1 & 11100 & $\mathrm{msu}$ & 0.20 & 10 & 0 & 1 & 1 & 1 & 1 & ok \\
\hline & two-tuneA-rau- $116-02+02$ & $\mathrm{MT}^{*}$ & 1 & -1 & 11100 & $\mathrm{msu}$ & - & - & 0 & 0 & 0 & 0 & 0 & ok \\
\hline 194 & two-tuneA-rau-116-03+01 & $\mathrm{MT}^{*}$ & 1 & -1 & 11600 & $\mathrm{su}$ & - & - & 0 & 0 & 0 & 0 & 0 & EccRF \\
\hline 195 & two-tuneA-rau-116-04+01 & - & 1 & -1 & 13080 & $\mathrm{su}$ & - & - & 0 & 0 & 0 & 0 & 0 & nDir, EccRF \\
\hline \multirow[t]{2}{*}{196} & two-tuneA-rau-117-01+01 & $\mathrm{MT}^{*}$ & -1 & 1 & 10610 & $\mathrm{msu}$ & - & - & 0 & 0 & 0 & 0 & 0 & $\mathrm{Mu}$ \\
\hline & two-AM-rau-117-01+01 & MT & -1 & 1 & 10610 & $\mathrm{msu}$ & - & - & 0 & 0 & 0 & 0 & 0 & MU \\
\hline \multirow[t]{2}{*}{197} & two-tuneA-rau-117-02+01 & MT & -1 & 1 & 10690 & $\mathrm{su}$ & - & - & 0 & 0 & 0 & 0 & 0 & ok \\
\hline & two-AM-rau-117-02+01 & MT & -1 & 1 & 10690 & $\mathrm{su}$ & 0.95 & 16.9 & 0 & 1 & 1 & 0 & 0 & ok \\
\hline 198 & two-AM-rau-117-03+01 & MT & -1 & 1 & 11100 & $\mathrm{msu}$ & - & - & 0 & 0 & 0 & 0 & 0 & MU \\
\hline 199 & two-tuneA-rau-118-01+01 & $\mathrm{MT}^{*}$ & -4 & -3 & 7100 & $\mathrm{su}$ & - & - & 0 & 0 & 0 & 0 & 0 & ok \\
\hline 200 & two-tuneA-rau-119-01+01 & $\mathrm{MT}^{*}$ & -3 & 3 & 9380 & $\mathrm{msu}$ & - & - & 0 & 0 & 0 & 0 & 0 & ok \\
\hline 201 & two-tuneA-rau-119-02+01 & $\mathrm{MT}^{*}$ & -3 & 3 & 9700 & $\mathrm{msu}$ & - & - & 0 & 0 & 0 & 0 & 0 & ok \\
\hline 202 & two-tuneA-rau-119-03+01 & $\mathrm{MT}^{*}$ & -3 & 3 & 10000 & $\mathrm{mu}$ & - & - & 0 & 0 & 0 & 0 & 0 & $\mathrm{MU}$ \\
\hline \multirow[t]{2}{*}{203} & two-tuneA-rau-119-04+01 & $\mathrm{MT}^{*}$ & -3 & 3 & 10480 & $\mathrm{su}$ & - & - & 0 & 0 & 0 & 0 & 0 & $\mathrm{ok}$ \\
\hline & two-AM-rau-119-04+01 & $\mathrm{MT}^{*}$ & -3 & 3 & 10480 & $\mathrm{su}$ & - & - & 0 & 0 & 0 & 0 & 0 & LowN \\
\hline
\end{tabular}




\section{Acknowledgements}

This project could be accomplished only with the outstanding and generous support and feedback of Stefan Treue whom I am deeply grateful for supervising the work and establishing an exceptional perspective on highest quality research, and also for his supportive and constructive role throughout the course of the thesis and the presentations of its data.

I owe a tremendous amount to several colleagues in the Cognitive Neuroscience Lab at the German Primate Center in Göttingen: First and most importantly, I like to acknowledge the exceptional technical and methodological advice and motivational support that came from Florian Pieper. Valuable discussions with Steffen Katzner and Laura Busse, and extremely fruitful and long debates with Tzvetomir Tzvetanov provided a lively source for continuous thoughts about the underlying scientific significance of the work. I also highly appreciate the substantial organisational and personal support from Sabine Stuber, the contribution of the graphical giftedness, MacIntosh-endowement and constructive optimism of Ralf Rode-Brockhausen, and the continuous availability for professional lab-, medical-, and animal-handling assistence of Dirk Prüsse.

I am also thankful to all other members of the lab and its surroundings who contributed to discussions and to solutions of sometimes quite intractable problems while proceeding with the scientific work, including: Robert Niebergall, Katharina Anton-Erxleben, Kurt Fahrner, Julio Martinez-Trujillo, Jan Churan, Peter Dicke, Dieter Patzwahl, Pinar Boyraz, Daniel Kaping, Danilo Musella, Suzanne Rensing, Annette Schrot, and Uwe Schönmann.

Last, but not least in the row of work-relate contributors I also will deeply thank Prof. Jürgens for his generousity to enable histological reconstruction, for his earnest corrections on the (originally suggested) final thesis version and for his patience during the thesis examination. He left a permanent impression of honoured scientific work from which I will benefit.

I also will gratefully acknowledge the complete confidence of my parents, Inge und Günter Womelsdorf, whose generousity allowed my curiousity to be pursued and to gain most valuable scientific insights.

Finally, the success of this thesis is fundamentally grounded in the patience, 
confidence, and support of Ulrike, whom I can not thank enough. 


\section{Bibliography}

Ahissar, M. and S. Hochstein (2000). The spread of attention and learning in feature search: effects of target distribution and task difficulty. Vision Research 40(10): 1349-1364.

Albrecht, D.G. and D.B. Hamilton (1982). Striate cortex of monkey and cat: contrast response function. ournal of Neurophysiology 48: 217-237.

Albright, T.D. (1984). Direction and orientation selectivity of neurons in visual area MT of the macaque. Journal of Neurophysiology 52: 1106-1130.

Albright, T.D. and R. Desimone (1987). Local precision of visuotopic organization in the middle temporal area (MT) of the macaque. Experimental Brain Research 65(3): 582-592.

Albright, T.D. and G.R. Stoner (1995). Visual motion perception. Proceedings of the National Academy of Science USA 92: 2433-2440.

Allman, J and F. Miezin (1985). Direction- and velocity-specific responses from beyond the classical receptive field in the middle temporal visual area (MT). Perception 14: 105-126.

Andersen, R.A. (1995). Coordinate transformations and motor planning in posterior parietal cortex. In Gazzaniga, M., editor, The Cognitive Neurosciences, chapter 33, pp. 519-532. MIT Press.

Andersen, R.A. and D.C. Bradley (1998). Perception of three-dimensional structure from motion. Trends in Cognitive Science 6(2): 222-228.

Andersen, R.A., G.K. Essick, and R.M. Siegel (1985). Encoding of spatial location by posterior parietal neurons. Science 230: 456-458.

Andersen, R.A., L.H. Snyder, and D.C. Bradley (1997). Multimodal representation of space in the posterior parietal cortex and its use in planning movements. Annual Reviews in Neuroscience 20: 303-330. 
Angelucci, A. and J.B. Levitt (2002). Anatomical origins of the classical receptive field and modulatory surround field of single neurons in macaque visual cortical area V1. In Azmitia, E.C., J. DeFelipe, and J.P. Rakic, editors, Progress in Brain Research, Vol. 136, pp. 273-388. Elsevier Science B.V., Amsterdam.

Armstrong-James, M. and M.J. George (1988). Influence of anesthesia on spontaneous activity and receptive field size of single units in rat sm1 neocortex. Experimental Neurology 99(2): 369-387.

Assad, J.A. (2003). Neural coding of behavioral relevance in parietal cortex. Current Opinion in Neurobiology 13: 194-197.

Azzopardi, P., M. Fallah, C.G. Gross, and H.R. Rodman (2003). Response latencies of neurons in visual areas MT and MST of monkeys with striate cortex lesions. Neuropsychologia 41(13): 1738-1756.

Bahcall, D.O. and E. Kowler (1999). Attentional interference at small spatial separations. Vision Research 39: 71-86.

Bair, W. and C. Koch (1996a). Temporal precision of spike trains in extrastriate cortex of the behaving macaque monkey. Neural Computation 8: 1184.

Bair, W. and C. Koch (1996b). Temporal precision of spike trains in extrastriate cortex of the behaving macaque monkey. Neural Computation 8: 1184.

Bair, W. and L.P. O'Keefe (1998). The influence of fixational eye movements on the response of neurons in area MT of the macaque. Visual Neuroscience 15(4): 779-786.

Barash, S., R. M. Bracewell, L. Fogassi, J. W. Gnadt, and R. A. Andersen (1991). Saccade-related activity in the lateral intraparietal area II. spatial properties. Journal of Neurophysiology 66(3): 1109-1124.

Barlow, H.B. (1953). Summation and inhibition in the frog's retina. Journal of Physiology 119: 69-78.

Beauchamp, M.S., R.W. Cox, and E.A. DeYoe (1997). Graded effects of spatial and featural attention on human area MT and associated motion processing areas. Journal of Neurophysiology 78: 516-520.

Behrmann, M. and C. Haimson (1999). The cognitive neuroscience of visual attention. Current Opinion in Neurobioloy 9(2): 158-163.

Ben Hamed, S. and J.R. Duhamel (2002). Ocular fixation and visual activity in the monkey intraprietal area. Experimental Brain Research 142: 512-528. 
Ben Hamed, S., J.R. Duhamel, and F. Bremmer (1997). Attentional modulation of visual receptive fields in the posterior parietal cortex of the behaving macaque. In Thier, P. and H.O. Karnath, editors, Parietal lobe contributions to orientation in 3D space, pp. 371-384. Springer-Verlag, Berlin.

Ben Hamed, S., J.R. Duhamel, and F. Bremmer (2002). Visual receptive field modulation in the lateral intraprietal area during attentive fixation and free gaze. Cerebral Cortex 12: 234-245.

Ben Hamed, S., J.R. Duhamel, F. Bremmer, and W. Graf (2001). Representation of the visual field in the lateral intraparietal area of macaque monkeys: a quantitative receptive field analysis. Experimental Brain Research 140: 127-144.

Bender, D.B. and M. Youakim (2001). Effect of attentive fixation in macaque thalamus and cortex. Journal of Neurophysiology 85: 219-234.

Bennett, P.J. and J. Pratt (2001). The spatial distribution of inhibition of return. Psychological Science 12(1): 76-80.

Bisley, J.W. and T. Pasternak (2000). The multiple roles of visual cortical areas $\mathrm{mt} / \mathrm{mst}$ in remebering the direction of visual motion. Cerebral Cortex 10: 1053-1065.

Blaser, E. and G. Sperling (1999). Measuring the amplification of attention. Proceedings of the National Academy of Science, USA 96: 11681-11686.

Blatt, G.J. and R.A. Andersen (1990). Visual receptive field organisation and cortico-cortical connections of the intraparietal area (area LIP) in the macaque. Journal of Comparative Neurology 299: 421-445.

Borghuis, B.G., J.A. Perge, I. Vajda, R.J. Wezel, W.A. Grind, and M.J. Lankheet (2003). The motion reverse correlation (MRC) method: A linear systems approach in the motion domain. Journal of Neuroscience Methods 123: 153-166.

Born, R.T. (2000). Center-surround interactions in the middle temporal visual area of the owl monkey. Journal of Neurophysiology 84: 2658-2669.

Born, R.T. and R.B.H. Tootell (1992). Segregation of global and local motion processing in primate middle temporal visual area. Nature 357: 497-499.

Boussaoud, D., L.G. Ungerleider, and R. Desimone (1990). Pathways for motion analysis: cortical connections of the medial superior temporal and fundus of the superior temporal visual areas in the macaque. Journal of Comparative Neurology 296: 462-495. 
Bradley, D.C. and R.A. Andersen (1998). Center-surround antagonism based on disparity in primate area mt. Journal of Neuroscience 18(18): 7552-7565.

Brefcynski, L.A. and E.A. DeYoe (1999). A physiologcal correlate of the spotlight of visual attention. Nature Neuroscience 2(4): 370-374.

Bringuier, V., F. Chavane, L. Glaeser, and Y. Fregnac (1999). Horizontal propagation of visual activity in the synaptic integration field of area 17 neurons. Science 283: 695-699.

Britten, K. H., W. T. Newsome, M. N. Shadlen, S. Celebrini, and J. A Movshon (1996). A relationship between behavioral choice and the visual responses of neurons in macaque MT. Visual Neuroscience 13(1): 87-100.

Britten, K. H., M. N. Shadlen, W. T. Newsome, and J. A Movshon (1992). The analysis of visual motion: A comparison of neuronal and psychophysical performance. Journal of Neuroscience 12(12): 4745-4765.

Britten, K.H. and H.W. Heuer (1999). Spatial summation in the receptive fields of MT neurons. Journal of Neuroscience 19(12): 5074-5084.

Britten, K.H. and R.J.A. Wezel (1998). Electrical microstimulation of cortical area MST biases heading perception in monkeys. Nature Neuroscience 1(1): 59-63.

Brotchie, P.R., R.A. Andersen, L.H. Snyder, and S.J. Goodman (1995). Head position signals used by parietal neurons to encode locations of visual stimuli. $\mathrm{Na}$ ture 375: 232-235.

Buracas, G.T., A.M. Zador, M.R. DeWeese, and T.D. Albright (1998). Efficient discrimination of temporal pattern by motion-sensitive neurons in primate visual cortex. Neuron 20: 959-969.

Cameron, E.L., J.C. Tai, and M. Carrasco (2002). Covert attention affects the psychometric function of contrast sensitivity. Vision Research 42: 949-967.

Caputo, G. and S. Guerra (1998). Attentional selection by distractor suppression. Vision Research 38: 669-689.

Carrasco, M., S. Ling, and S. Read (2004). Attention alters appearance. Nature Neuroscience 7(3): 308-313.

Carrasco, M. and C. Penpeci-Talgar (2000). Spatial covert attention increases contrast sensitivity across the csf: support for signal enhancement. Vision Research 40: 1203-1215. 
Carrasco, M., P. Williams, and Y. Yeshurun (2002). Covert attention increases spatial resolution with and without masks: Support for signal enhancement. Journal of Vision 2: 467-479.

Carrasco, M. and Y. Yeshurun (1998). The contribution of covert attention to the set-size and eccentricity effects in visual search. Journal of Experimental Psychology: Human Percpetion \& Performance 24: 673-692.

Castiello, U. and C. Umilta (1990). Size of the attentional focus and efficiency of processing. Acta Psychologica 73: 195-209.

Castro-Alamancos, M.A. (2002). V1 neurons signal acquisition of internal representation of stimulus location. Journal of Neuroscience 22(22): 9651-9655.

Cave, G. and S. Zimmerman (1997). Flexibility in spatial attention before and after practice. Psychological Science 8: 399-403.

Cave, K. and N. Bichot (1999). Visuospatial attention: beyond a spotlight model. Psychonomic Bulletin \& Review 6: 204-223.

Cave, K.R. and S.M. Kosslyn (1989). Varieties of size-specific visual selection. Journal of Experimental Psychology: General 270: 764-769.

Celebrini, S. and W.T. Newsome (1994). Neuronal and psychophysical sensitivity to motion signals in extrastriate area MST of the macaque monkey. Journal of Neuroscience 14(7): 4109-4124.

Celebrini, S. and W.T. Newsome (1995). Microstimulation of extrastriate area MST influences performance on a direction discrimination task. Journal of Neurophysiology $73(2)$ : 437-448.

Chapman, B. and L.S. Stone (1996). Turning a blind eye to cortical receptive fields. Neuron 16: 9-12.

Chelazzi, L., J. Duncan, and E.K. Miller (1998). Responses of neurons in inferior temporal cortex during memory-guided visual search. Journal of Neurophysiology 80: 2918-2940.

Chelazzi, L., E.K. Miller, and J. Duncan (2001). Responses of neurons in macaque area V4 during memory-guided visual search. Cerebral Cortex 11: 761-772.

Chelazzi, L., E.K. Miller, J. Duncan, and R. Desimone (1993). A neural basis for visual searchin inferior temporal cortex. Nature 363: 345-347. 
Chino, Y.M. (1997). Receptive-field plasticity in the adult visual cortex: Dynamic signal rerouting or experience-dependent plasticity. Seminars in Neuroscience 9: 34-46.

Christ, R.E. and W. Li (2001). Learning to see: Experience and attention in primary visual cortex. Nature Neuroscience 4(5): 519-525.

Colby, C.L. (1996). A neurophysiological distinction between attention and intention. In Inui, T. and J.L. McClelland, editors, Attention and Performance, Vol. XVI, pp. 157-177. MIT Press, Cambridge, Massachusetts.

Colby, C.L. and M.E. Goldberg (1999). Space and attention in parietal cortex. Annual Reviews in Neuroscience 22: 319-349.

Collie, A., P. Maruff, M. Yucel, and J. Currie (2000). Spatiotemporal distribution of facilitation and inhibition of return arising from the reflexive orienting of covert attention. Journal of Experimental Psychology: Human Perception 8 Performance 26: 1733-1745.

Connor, C.E., J.L. Gallant, D.C. Preddie, and D.C. Essen (1996). Responses in area V4 depend on the spatial relationship between stimulus and attention. Journal of Neurophysiology 75: 1306-1308.

Connor, C.E., D.C. Preddie, J.L. Gallant, and D.C. Essen (1997). Spatial attention effects in macaque area V4. Journal of Neuroscience 17(9): 3201-3214.

Constantinidis, C. and M.A. Steinmetz (2001a). Neuronal responses in area 7a to multiple-stimulus displays: I. Neurons encode the location of the salient stimulus. Cerebral Cortex 11: 581-591.

Constantinidis, C. and M.A. Steinmetz (2001b). Neuronal responses in area 7a to multiple-stimulus displays: II. Responses are suppressed at the cued location. Cerebral Cortex 11: 592-597.

Cook, E. and J.H.R. Maunsell (2002a). Dynamics of neuronal responses in macaque MT and VIP during motion detection. Nature Neuroscience 10(5): 985-994.

Cook, E.P. and J.H.R. Maunsell (2002b). Attentional modulation of behavioral performance and neuronal responses in middle temporal and ventral intraprietal areas of the macaque monkey. Journal of Neuroscience 22(5): 1994-2004.

Corbetta, M. (1998). Frontoparietal networks for directing attention and the eye to visual locations: identical, independent, or overlapping neural systems? Proceedings of the National Academy of Science USA 95: 831-838. 
Corbetta, M. and G.L. Shulman (1998). Human cortical mechanisms of visual attention during orienting and search. Philosophical Transactions of the Royal Society London, BS 353: 1353-1362.

Corbetta, M. and G.L. Shulman (2002). Control of goal-directed and stimulus-driven attention in the brain. Nature Neuroscience Reviews 3: 201-215.

Coull, J.T. (1998). Neural correlates of attention and arousal: insights from electrophysiology, functional neuroimaging and psychopharmacology. Progress in Neurobiology 55: 343-361.

Culham, J.C. and N.G. Kanwisher (2001). Neuroimaging of cognitive functions in human parietal cortex. Current Opinion in Neurobiology 11: 157-163.

Cutzu, F. and J.K. Tsotsos (2003). The selective tuning model of attention: psychophysical evidence for a suppressive annulus around an attended item. Vision Research 43: 205-219.

Darian-Smith, C. and C.D. Gilbert (1995). Topographic reorganization in the striate cortex of the adult cat and monkey is cortically mediated. Journal of Neuroscience 15(3): 1631-1647.

Das, A. (1997). Plasticity in adult visual cortex: a review. Network: Computational Neural Systems 8: 33-76.

Das, A. and C.D. Gilbert (1995). Receptive field expansion in adult visual cortex is linked to dynamic changes in strength of cortical connections. Journal of Neurophysiology 2: 779-792.

Dato, T. (1989). Interactions of visual stimuli in the receptive fields of inferior temporal neurons in awake monkeys. Experimental Brain Research 77: 23-30.

De Angelis, G.C., A. Anzai, and I. Ohzawa (1995). Receptive field structure in the visual cortex: Does selective stimulation induce plasticity. Proceedings of the National Academy of Science USA 92: 9682-9686.

De Angelis, G.C., B.C. Cumming, and W.T. Newsome (1998). Cortical area MT and the perception of stereoscopic depth. Nature 394: 677-680.

De Angelis, G.C., G.M. Ghose, I. Ohzawa, and R.D. Freeman (1999). Functional micro-organisation of primary visual cortex: recpetive field analysis of nearby neurons. Journal of Neuroscience 19(9): 4046-4064.

De Angelis, G.C. and W.T. Newsome (1999). Organization of disparity-selective neurons in macaque area mt. Journal of Neuroscience 19(4): 1398-1415. 
De Angelis, G.C. and I. Ohzawa (1993). Spatial organisation of simple-cell receptive fields in the cat's striate cortex. I. General characterstics and postnatal development. Journal of Neurophysiology 69(4): 1091-1117.

De Angelis, G.C., I. Ohzawa, and R.D. Freeman (1995). Receptive-field dynamics in the central visual pathways. Trends in Neuroscience 18(10): 451-458.

De Weerd, P., R. Gattass, and R. Desimone (1995). Responses of cells in monkey visual cortex during perceptual filling-in of an artificial scotoma. $N a$ ture 377: 731-734.

Dean, A.F. (1991). The variability of discharge of simple cells in the cat striate cortex. Experimental Brain Research 44: 437-440.

Deco, G. and B. Schuermann (2000). A hierarchical neural system with attentional top-down enhancement of the spatial resolution for object recognition. Vision Research 40: 2845-2859.

Deco, G. and J. Zihl (2001a). A neurodynamical model of visual attention: Feedback enhancement of spatial resolution in a hierarchical system. Journal of Computational Neuroscience 10: 231-253.

Deco, G. and J. Zihl (2001b). Top-down selective visual attention: A neurodynamical approach. Visual Cognition 8: 119-140.

Desimone, R. (1998). Visual attention mediated by biased competition in extrastriate visual cortex. Philosophical Transactions of the Royal Society London, BS 353: 1245-1255.

Desimone, R. and J. Duncan (1995). Neural mechanisms of selective visual attention. Annual Reviews in Neuroscience 18: 193-222.

Desimone, R. and L.G. Ungerleider (1986). Multiple visual areas in the caudal superior temporal sulcus of the macaque. Journal of Comparative Neurology 248: 164-189.

DiCarlo, J.J. and H.R. Maunsell (2003). Anterior inferotemporal neurons of monkeys engaged in object recognition can be highly sensitive to object retinal position. Journal of Neurophysiology 89: 3264-3278.

Ditterich, J., M. Mazurek, and M.N. Shadlen (2003). Microstimulation of visual cortex affects the speed of perceptual decisions. Nature Neuroscience 6(8): 891-898. 
Dodd, J.V., K. Krug, B.G. Cumming, and A.J. Parker (2001). Perceptually biastable three-dimensional figures evoke high choice probabilities in cortical area mt. Journal of Neuroscience 21: 4809-4821.

Downing, C.J. (1988). Expectancy and visuo-spatial attention: Effects on perceptual quality. Journal of Experimental Psychology: Human Perception and Performance 14: 188-202.

Downing, C.J. and S. Pinker (1985). The spatial structure of visual attention. In Posner, M.I. and O.S.M. Marin, editors, Attention and Performance XI, pp. 251-265. Erlbaum, Hillsdale, NJ.

Duhamel, J.R., C.L. Colby, and M.E. Goldberg (1992). The updating of the representation of visual space in parietal cortex by intended eye movements. Science 255: 90-92.

Duncan, J. (1984). Selective attention and the organisation of visual information. Journal of Experimental Psychology: General 4: 501-517.

Duncan, J. and G. Humphreys (1989). Visual search and stimulus similarity. Psychological Review 96: 433-458.

Duncan, R.O., T.D. Albright, and G.R. Stoner (2000). Occlusion and the interpretation of visual motion: Perceptual and neuronal effects of context. Journal of Neuroscience 20(15): 5885-5897.

Eckhorn, R., F. Krause, and J.I. Nelson (1993). The RF-cinematogram. a crosscorrelation technique for mapping several visual receptive fields at once. Biological Cybernetics 69(1): 37-55.

Egeth, H.E. and S. Yantis (1997). Visual attention: control, representation, and time course. Annual Review in Psychology 48: 269-297.

Eifuku, S. and R.H Wurtz (1999). Response to motion in extrastriate area MSTl: Disparity sensitivity. Journal of Neurophysiology 82: 2462-2475.

Eriksen, C.W. and J.D. St.James (1986). Visual attention within and around the field of focal attention: A zoom lens model. Perception \& Psychophysics 40(4): 225-240.

Eskandar, E.N. and J.A. Assad (1999). Dissociation of visual, motor and predictive signals in parietal cortex during visual guidance. Nature Neuroscience 2(1): 88-93. 
Eskandar, E.N. and J.A. Assad (2002). Distinct nature of directional signals among parietal cortical areas during visual guidance. Journal of Neurophysiology 88(4): 1777-1790.

Everling, S., C.J. Tinsley, and D. Gaffan (2002). Filtering of neural signals by focused attention in the monkey prefrontal cortex. Nature Neuroscience 5(7): 671-676.

Eysel, U.T. and G. Schweigart (1999). Increased receptive field size in the surround of chronic lesions of adult cat visual cortex. Cerebral Cortex 9: 101-109.

Eysel, U.T., G. Schweigart, T. Mittmann, D. Eyding, Y. Qu, F. Vandesande, G. Orban, and L. Arckens (1999). Reorganization in the visual cortex after retinal and cortical damage. Restorative Neurolology and Neuroscience 15(2): 153-164.

Felleman, D. J. and J. H. Kaas (1984). Receptive-field properties of neurons in middle temporal visual area (MT) of owl monkeys. Journal of Neurophysiology 52(3): 488-513.

Fellemann, D.J. and D.C. Essen (1991). Distributed hierarchical processing in the primate cerebral cortex. Cerebral Cortex 1: 1-47.

Ferrera, V. P. and S. G. Lisberger (1997). Neuronal responses in visual areas MT and MST during smooth pursuit target selection. Journal of Neurophysiology 78(3): 1433-1446.

Ferrera, V.P., K.K. Rudolphs, and J.H.R. Maunsell (1994). Responses of neurons in the parietal and temporal visual pathways during a motion task. Journal of Neuroscience 14(10): 6171-6186.

Ffytche, D.H., C.N. Guy, and S. Zeki (1995). The parallel visual motion inputs into areas V1 and V5 of human cerebral cortex. Brain 118: 1375-1394.

Freedman, D.J., M. Riesenhuber, T. Poggio, and E.K. Miller (2003). A comparison of primate prefrontal and inferotemporal cortices during visual categorization. Journal of Neuroscience 23(12): 5235-5246.

Freeman, T.C., S. Durand, D.C. Kiper, and M. Carandini (2002). Suppression without inhibition in visual cortex. Neuron 35(4): 759-771.

Fries, W. (1981). The projection from lateral geniculate nucleus to the prestriate cortex of the macaque monkey. Proceedings of the Royal Society of London, BS 213: 73-86. 
Fritz, J., S. Shamma, M. Elhilali, and D. Klein (2003). Rapid task-related plasticity of spectrotemporal receptive fields in primary auditory cortex. Nature Neuroscience 6(11): 1216-1223.

Fu, Y.X., K. Djupsund, H. Gao, B. Hayden, and Y. Shen, K. Dan (2002). Temporal specificity in the cortical plasticity of visual space representation. Science 296: 1999-2003.

Gandhi, S.P. and D.J. Heeger (1999). Spatial attention affects brain activity in human primary visual cortex. Proceedings of the National Academy of Science USA 96: 3314-3319.

Gandolfo, F., C.S. Li, P.J. Benda, C. Pandoa-Sciuppa, and E. Bizzi (2000). Cortical correlates of learning in monkeys adapting to a new dynamical environment. Proceedings of the National Academy of Science, USA 97: 2259-2263.

Gardner, J.L. and S.G. Lisberger (2002). Serial linkage of target selection for orienting and tracking eye movements. Nature Neuroscience 5(9): 892-899.

Gattass, R. and C.G. Gross (1981). Visual topography of striate projection zone (MT) in posterior superior temporal sulcus of the macaque. Journal of Neurophysiology 46(3): 621-638.

Gattass, R., A.P.B. Sousa, M. Mishkin, and L.G. Ungerleider (1997). Cortical projections of area V2 in the macaque. Ceebral Cortex 7: 110-129.

Gautama, T. and M.M. Van Hulle (2001). Functions of center-surround antagonism for motion in visual area MT/V5: a modeling study. Vision Research 41: 3917-3930.

Gilbert, C., M. Ito, and M. Kapadia (2000). Interactions between attention, context and learning in primary visual cortex. Vision Research 40: 1217-1226.

Gilbert, C.D. (1992). Horizontal integration and cortical dynamics. Neuron 9: 1-13.

Gilbert, C.D. (1995). Dynamic properties of adult visual cortex. In Gazzaniga, M., editor, The Cognitive Neuroscience, chapter 5, pp. 73-90. MIT Press.

Girard, P. and P.A. Salin (1991). Visual activity in areas V3A and V3 during reversible inactivation of area V1 in the macaque monkey. Journal of Neurophysiology 66: 1493-1503.

Goldberg, M.E. and M.A. Segraves (1987). Visuospatial and motor attention in the monkey. Neuropsychologia 25: 107-118. 
Gonzalez, F., R. Perez, and M.S. Justo (2001). Receptive-field organisation of disparity-sensitive cells in macaque medial superior temporal cortex. European Journal of Neuroscience 14: 167-173.

Goodale, M.A. and A.D. Milner (1992). Separate visual pathways for perception and action. Trends in Neuroscience 15(1): 20-25.

Gottlieb, J. (2002). Parietal mechanisms of target representation. Current Opinion in Neurobiology 12(2): 134-140.

Gottlieb, J.P. and M.E. Goldberg (1999). Activity of neurons in the lateral intraparietal area of the monkey during an antisaccade task. Nature Neuroscience 2(10): 906-912.

Gottlieb, J.P., M. Kusunoki, and M.E. Goldberg (1998). The representation of visual salience in monkey parietal cortex. Nature 391: 481-484.

Graziano, M.S.A., R.A. Andersen, and R.J. Snowden (1994). Tuning of MST neurons to spiral motions. Journal of Neuroscience 14(1).

Grinvald, A., E. E. Lieke, R. D. Frostig, and R. Hildesheim (1994). Cortical point-spread function and long-range lateral interactions revealed by real-time optical imaging of macaque monkey primary visual cortex. Journal of Neuroscience 14(5): 2545-2568.

Haenny, P.E. and P.H. Schiller (1987). State dependent activity in monkey visual cortex I. Single cell activity in V1 and V4 on visual tasks. Experimental Brain Research 69: 225-244.

Handy, T.C., A. Klingstone, and G.R. Mangun (1996). Spatial distribution of visual attention: Perceptual sensitivity and response latency. Perception \& Psychophysics 58(4): 618-627.

Hawkins, H.L., S.A. Hillyard, S.J. Luck, M. Mouloua, C.J. Downing, and D.P. Woodward (1990). Visual attention modulates signal detectability. Journal of Experimental Psychology: Human Perception \& Performance 16(4): 802-811.

He, S. and P. Cavanagh (1997). Attentional resolution. Trends in Cognitive Sciences 1(3): 115-120.

He, S., P. Cavanagh, and J. Intriligator (1996). Attentional resolution and the locus of visual awareness. Nature 383: 334-337. 
Heeger, D.J., S.P. Gandhi, and A.C. Huk (2001). Neuronal correlates of attention in human visual cortex. In Braun, J. and C. Koch, editors, Visual attention and cortical circuits, pp. 25-48. MIT Press, Cambridge, Massachusetts, 1st edition.

Heeger, D.J. and E.P. Simoncelli (1996). Computational models of cortical visual processing. Proceedings of the National Academy of Science USA 93: 623-627.

Henderson, J.M. (1991). Stimulus discrimination following covert attentional orienting to an exogenous cue. Journal of Experimental Psychology: Human Perception E) Performance 17(1): 91-106.

Henderson, J.M. (1996). Spatial precues affect target discrimination in the absence of visual noise. Journal of Experimental Psychology: Human Perception \& Performance 22(3): 780-787.

Henderson, J.M. and A.D. Macquistan (1993). The spatial distribution of attention following an exogenous cue. Perception \& Psychophysics 53(2): 221-230.

Heuer, H.W. and K. Britten (2004). Optic flow signals in extrastriate area mst: Comparison of perceptual and neuronal selectivity. Journal of Neurophysiology 91: 1314-1426.

Hillyard, S.A., E.K. Vogel, and S.J. Luck (1998). Sensory gain control (amplification) as a mechanism of selective attention: electrophysiological and neuroimaging evidence. Philosophical Transactions of the Royal Society London, BS 353: 1257-1270.

Hochstein, S. and M Ahissar (2002). View from the top: hierarchies and reverse hierarchies in the visual system. Neuron 36(5): 791-804.

Hughes, H.C. and L.D. Zimba (1985). Spatial maps of directed visual attention. Journal of Experimental Psychology: Human Perception and Performance 11: 409-430.

Intriligator, J. and P. Cavanagh (2001). The spatial resolution of visual attention. Cognitive Psychology 43: 171-216.

Iriki, A., M. Tanaka, and Y. Iwamura (1996). Coding of modified body schema during tool use by macaque postcentral neurons. Neuroreport. 7(14): 2325-2330.

Ito, M. and C.D. Gilbert (1999). Attention modulates contextual influences in the primary visual cortex of alert monkeys. Neuron 22: 593-604.

Ito, M. and G. Westheimer (1998). Attention and perceptual learning modulate contextual influences on visual perception. Neuron 20: 1191-1197. 
Itti, L. and C. Koch (2001). Computational modelling of visual attention. Nature Neuroscience Reviews 2: 194-203.

Judge, S.J., B.J. Richmond, and F.C. Shu (1980). Implantation of magnetic search coils for measurement of eye position: An improved method. Vision Research 20: 535-537.

Kaas, J.H. (1991). Plasticity of sensory and motor maps in adult mammals. Annual Reviews in Neuroscience 14: 137-167.

Kahneman, D. (1973). Attention and Effort. Englewoods Cliff, NJ: Prentice Hall, 1st edition.

Kaiser, M. and M. Lappe (2004). Perisaccadic mislocalization orthogonal to saccade direction. Neuron 41: 293-300.

Kalarickal, G.J. and J.A. Marshall (1999). Models of receptive-field dynamics in visual cortex. Visual Neuroscience 16(6): 1055-1081.

Kanwisher, N. and E. Wojciuluk (2000). Visual attention: Insight from brain imaging. Nature Neuroscience Reviews 1: 91-100.

Kapadia, M.K. and G. Westheimer (1999). Dynamics of spatial summation in primary visual cortex of alert monkeys. Proceedings of the National Academy of Science USA 96: 12073-12078.

Kastner, S., P. De Weerd, and R. Desimone (1998). Mechanisms of directed attention in the human extrastriate cortex as revealed by functional mri. Science 282: 108-111.

Kastner, S., P. De Weerd, M.A. Pinsk, M.I. Elizondo, and R. Desimone (2001). Modulation of sensory suppression: implications for receptive field sizes in the human visual cortex. Journal of Neurophysiology 86: 1398-1411.

Kastner, S., M.A. Pinsk, P. De Weerd, and R. Desimone (1999). Increased activity in human visual cortex during directed attention in the absence of visual stimulation. Neuron 22: 751-761.

Kastner, S. and L.G. Ungerleider (2000). Mechanisms of visual attention in the human cortex. Annual Reviews in Neuroscience 23: 315-341.

Kastner, S. and L.G. Ungerleider (2001). The neural basis of biased competition in human visual cortex. Neuropsychologia 39: 1263-1276.

Kim, M.S. and K.R. Cave (1995). Spatial attention invisual search for features and conjunctions. Psychological Science 6: 376-380. 
Koch, C. and S. Ullman (1985). Shifts in selective visual attention: Towards the underlying neural circuitry. Human Neurobiology 4: 219-227.

Kohn, A. and J.A. Movshon (2003). Neuronal adaptation to visual motion in area MT of the macaque. Neuron 39: 681-691.

Kourtzi, Z., H.H. Buelthoff, and M. Erb (2002). Object-selective responses in the human motion area mt/mst. Nature Neuroscience 5(1): 17-18.

Krekelberg, B., M. Kubischik, K.P. Hoffmann, and F. Bremmer (2003). Neural correlates of visual localization and perisaccadic mislocalization. Neuron 37: 537-545.

Kuffler, S.W. (1953). Discharge patterns and functional organisation of the mammalian retina. Journal of Neurophysiology 16: 37-68.

Kusunoki, M. and M.E. Goldberg (2003). The time course of perisaccadic receptive field shifts in the lateral intraprietal area of the monkey. Journal of Neuropysiology 89: 1519-1527.

Kusunoki, M., J. Gottlieb, and M.E. Goldberg (2000). The lateral intraparietal area as a salience map: the representation of abrupt onset, stimulus motion, and task relevance. Vision Research 40: 1459-1468.

LaBerge, D. (1983). Spatial extent of attention to letters and words. Journal of Experimental Psychology: Human Perception \& Performance 9: 371-379.

LaBerge, D. (1995). Attentional processing: The brain's art of mindfulness. Harvard University Press, Cambridge MA.

LaBerge, D. (2000). Networks of attention. In Gazzaniga, M., editor, The Cognitive Neurosciences, chapter 49, pp. 711-724. MIT Press.

LaBerge, D. and V. Brown (1989). Theory of attentional operations in shape identification. Psychological Review 96: 101-124.

LaBerge, D., R.L. Carlson, J.K. Williams, and B.G. Bunney (1997). Shifting attention in visual space: Tests of moving-spotlight models versus an activitydistribution model. Journal of Experimental Psychology: Human Perception 6 Performance 23(5): 1380-1392.

Lagae, L., B. Gulyas, S. Raiguel, and G.A Orban (1994a). Laminar analysis of motion information processing in macaque V5. Brain Research 496: 361-367.

Lagae, L., H. Maes, S. Raiguel, D.K. Ziao, and G.A. Orban (1994b). Responses of macaque sts neurons to optic flow components: A comparison of area MT and MST. Journal of Neurosphysiology 71(5): 1597-1626. 
Lamy, D., Y. Tsal, and H.E. Egeth (2003). Does a salient distractor capture attention early in processing? Psychonomic Bulletin \& Review 10(3): 621-629.

Lavie, N. and J. Driver (1996). On the spatial extent of attention in object-based visual selection. Perception \& Psychophysics 58(8): 1238-1251.

Lavie, N. and Y. Tsal (1994). Perceptual load as the major determinant of the locus of selection in visual attention. Perception $\mathscr{E}$ Psychophysics 56: 183-197.

Lee, T.S., C.F. Yang, and R.D. Romero (2002). Neural activity in early visual cortex reflects behavioral experience and higher-order percpetual saliency. Nature Neuroscience pp. 1-9.

Leopold, D.A. and N.K. Logothetis (1998). Microsaccades differentially modulate neural activity in the striate and extrastriate visual cortex. Experimental Brain Research 123(3): 341-345.

Leopold, D.A. and N.K. Logothetis (1999). Multistable phenomena: changing views in perception. Trends in Cognitive Sciences 3(7): 254-264.

Levitt, J.B. and J.S. Lund (2002). The spatial extent over which neurons in macaque striate cortex pool visual signals. Visual Neuroscience 19(4): 439-452.

Li, Z. (2002). A saliency map in primary visual cortex. Trends in Cognitive Sciences 6(1): 9-16.

Liu, T., S.D. Slotnick, J.T. Serences, and S. Yantis (2003). Cortical mechanisms of feature-based attentional control. Cerebral Cortex 13: 1334-1343.

Livingstone, M.S., C.P. Pack, and R.T. Born (2001). Two-dimensional substructure of MT receptive fields. Neuron 30(3): 781-793.

Livingstone, M.S. and D.Y Tsao (1999). Receptive fields of disparity-selective neurons in macaque striate cortex. Nature Neuroscience 2(9): 825-832.

Logothetis, N.K. (1994). Physiological studies of motion inputs. In Smith, A.T. and R.J. Snowden, editors, Visual Detection of Motion, pp. 177-216. New York: Academic Press, New York.

Logothetis, N.K. and D.L. Sheinberg (1996). Visual object recognition. Annual Reviews in Neuroscience 19: 577-621.

Luck, S.J., L. Chelazzi, S.A. Hilljard, and R. Desimone (1997a). Neural mechanisms of selective attention in areas V1, V2, and V4 of macaque visual cortex. Journal of Neurophsiology 77: 24-42. 
Luck, S.J., M. Girelli, M.T. McDermott, and M.A. Ford (1997b). Bridging the gap between monkey neurophysiology and human perception: An ambiguity resolution theory of visual selective attention. Cognitive Psychology 33(1): 64-87.

Luck, S.J., S.A. Hillyard, M. Mouloua, M.G. Woldorff, V.P. Clark, and H.L. Hawkins (1996). Effects of spatial cueing on luminance detectability: psychophysical and electrophysiological evidence for early selection. Journal of Experimental Psychology: Human Perception 63 Performance 20(4): 887-904.

Lynch, J.C., V.B. Mountcastle, and W.H. Talbot (1977). Parietal lobe mechanisms for directed visual attention. Journal of Neurophysiology 40(2): 362-389.

Marcus, D.S. and D.C. Van Essen (2002). Scene segmentation and attention in primate cortical areas V1 and V2. Journal of Neurophysiology 88(5): 2648-2658.

Martin, K.A.C. (1994). A brief history of the feature detector! Cerebral Cortex 4: 1-7.

Martinez, A., L. Anllo-Vento, M.I. Sereno, L.R. Frank, R.B. Buxton, D.J. Dubowitz, Wongm E.C., H. Hinrichs, and H.J. Heinze (1999). Involvement of striate and extrastriate visual cortical areas in spatial attention. Nature Neurscience 2(4): 364-369.

Martinez, A., F. DiRusso, L. Anello-Vento, M.I. Sereno, R.B. Buxton, and S.A Hillyard (2001). Putting spatial attention on the map: timing and localization of stimulus selection processes in striate and extrastriate visual areas. Vision Research 41: 1437-1457.

Martinez-Conde, S., S.L. Macknik, and D.H. Hubel (2000). The role of fixational eye movements in visual perception. Nature Neuroscience Reviews 5(3): 229-240.

Martinez-Trujillo, J.C. and S. Treue (2002). Attentional modulation strength in cortical area MT depends on stimulus contrast. Neuron 35: 365-370.

Martinez-Trujillo, J.C. and S. Treue (2004). Feature-based attention increases the selectivity of population responses in primate visual cortex. Current Biology 14: 1-20.

Maunsell, J.H. and E.P. Cook (2002). The role of attention in visual processing. Philosophical Transactions of the Royal Society London, BS 357(1424): 1063-1072.

Maunsell, J.H. and C.J. McAdams (2001). Effects of attention on the responsiveness and selectivity of individual neurons in visual cerebral cortex. In Braun, J., C. Koch, and J.L. Davis, editors, Visual attention and cortical circuits, pp. 103-120. Cambridge, Massachusetts: MIT Press, Cambridge, Massachusetts. 
Maunsell, J.H. and D.C. Essen (1983). The connections of the middle temporal visual area (MT) and their relationship to a cortical hierarchy in the macaque monkey. Journal of Neuroscience 3: 2563-2586.

Maunsell, J.H.R. (1992). Functional visual streams. Current Opinion in Neurobiology 2: 506-510.

Maunsell, J.H.R. (1995). The brain's visual world: Representation of visual targets in cerebral cortex. Science 270: 764-769.

Maunsell, J.H.R. and V.P. Ferrera (1995). Attentional mechanisms in visual cortex. In Gazzaniga, M., editor, The Cognitive Neurosciences, chapter 28, pp. 451-461. MIT Press.

Maunsell, J.H.R. and C.J. McAdams (2000). Effects of attention on neuronal response properties in visual cerebral cortex. In Gazzaniga, M.S., editor, The New Cognitive Neurosciences, pp. 315-324. Cambridge, MA: MIT Press, Cambridge MA, 2nd edition.

Maunsell, J.H.R. and D.C. Essen (1983a). The connections of the middle temporal visual area (MT) and their relationship to a cortical hierarchy in the macaque monkey. Journal of Neuroscience 3(12): 2563-2586.

Maunsell, J.H.R. and D.C. Essen (1983b). Functional properties of neurons in the middle temporal visual area of the macaque monkey. II. binocular interaction and sensitivity to binocular disparity. Journal of Neurophysiology 49(5): 1148-1167.

Mazer, J.A. and J.L. Gallant (2003). Goal-related activity in V4 during free viewing visual search: Evidence for a ventral stream visual salience map. Neuron 40: 1241-1250.

Mazer, J.A., W.E. Vinje, J. McDermott, and P.H. Schiller (2002). Spatial frequency and orientation tuning dynamics in area V1. Proceedings of the National Academy of Science, USA 99(3): 1645-1650.

McAdams, C. and R.C. Reid (2003). Effects on attention on the spatial and temporal structure of receptive fields in macaque primary visual cortex. Society for Neuroscience, Annual Meeting .

McAdams, C.J. and J.H.R. Maunsell (1999). Effects of attention on orientationtuning functions of single neurons in macaque cortical area V4. Journal of Neuroscience 19(1): 431-441. 
McAdams, C.J. and J.H.R. Maunsell (2000). Attention to both space and feature modulates neuronal responses in macaque area V4. Journal of Neurophysiology 83: 1751-1755.

McMains, S.A. and D.C. Somers (2004). Multiple spotlights of attentional selection in human visual cortex. Neuron 42: 677-686.

Mesulam, M.M. (1999). Spatial attention and neglect: parietal, frontal and cingulate contributions to the mental representation and attentional targeting of salient extrapersonal events. Philosophical Transactions of the Royal Society London, BS 354: 1325-1346.

Mikami, A., W.T. Newsome, and R.H. Wurtz (1986). Motion selectivity in macaque visual cortex. Journal of Neurophysiology 55(6): 1308-1327.

Miller, E. K., P. M. Gochin, and C. G. Gross (1993). Supression of visual responses of neurons in inferior temporal cortex of the awake macaque by addition of a second stimulus. Brain Research 616: 25-29.

Missal, M. and R. Vogels (1997). Responses of macaque inferior temporal neurons to overlapping shapes. Cerebral Cortex 7: 758-767.

Miyashita, Y. and T. Hayashi (2000). Neural representation of visual objects: encoding and top-down activation. Current Opinion in Neurobiology 10: 187-194.

Moore, T. and K.M. Armstrong (2003). Visuomotor origins of covert spatial attention. Nature 421: 370-373.

Moore, T., K.M. Armstrong, and M. Fallah (2003). Visuomotor origins of covert spatial attention. Neuron 40: 671-683.

Moore, T. and M. Fallah (2001). Control of eye movements and spatial attention. Proceedings of the National Academy of Science USA 98: 1273-1276.

Moore, T. and M. Fallah (2004). Microstimulation of the frontal eye fields and its effects on covert spatial attention. Journal of Neurophysiology 91: 152-162.

Moore, T., H.R. Rodman, and C.G. Gross (2001). Direction of motion discrimination after early lesions of striate cortex ( v1) of the macaque monkey. Proceedings of the National Academy of Science USA 98(1): 325-330.

Moran, J. and R. Desimone (1985). Selective attention gates visual processing in the extrastriate cortex. Science 229: 782-784. 
Motter, B.C. (1993). Focal attention produces spatially selective processing in the visual cortical areas V1, V2, and V4 in the presence of competing stimuli. Journal of Neurophysiology 70(3): 909-919.

Motter, B.C. and V.B. Mountcastle (1981). The functional properties of the lightsensitive neurons of the posterior parietal cortex studied in waking monkeys: Foveal sparing and opponent vector organization. Journal of Neuroscience 1: 3-26.

Mountcastle, V. B. (1976). Then world around us: Neural command functions for selective attention. Neurosci. Res. Prog. Bull. 14: 1-47.

Mountcastle, V.B., R.A. Andersen, and B.C. Motter (1981). The influence of attentive fixation upon the excitability of light-sensitive neurons of the posterior parietal cortex. Journal of Neuroscience 1: 1218-1235.

Mountcastle, V.B., B.C. Motter, M.A. Steinmetz, and A.K. Sestokas (1987). Common and differential effects of attentive fixation on the excitability of parietal and prestriate (v4) cortical visual neurons in the macaque monkey. Journal of Neuroscience 7(7): 2239-2255.

Mounts, J.R.W. (2000a). Attentional capture by abrupt onsets and feature singletons produce inhibitory surrounds. Perception \& Psychophysics 62: 1485-1493.

Mounts, J.R.W. (2000b). Evidence for suppressive mechanisms in attentional selection: feature singletons produce inhibitory surrounds. Perception 63 Psychophysics 62: 969-983.

Movshon, J.A. and W.T. Newsome (1996). Visual response properties of striate cortical neurons projecting to area MT in macaque monkeys. Journal of Neuroscience 16(23): 7733-7741.

Mozer, M.C. and M. Sitton (1998). Computational modeling of spatial attention. In Pashler, H., editor, Attention, pp. 341-395. Psychology Press, East Sussex.

Müller, H.J. and G.W. Humphreys (1991). Lumiance-increment detection: Capacity limited or not? Journal of Experimental Psychology: Human Perception $\&$ Performance 17: 107-124.

Müller, M.M., T.W. Picton, P. Valdes-Sosa, J. Riera, W.A. Teder-Saelerjaervi, and S.A. Hillyard (1998). Effects of spatial attention on the steady-state visual evoked potential in the 20-28Hz range. Cognitive Brain Research 6: 249-261.

Müller, N.G., O. A. Bartelt, T.H. Donner, A. Villringer, and S.A. Brandt (2003). Physiological correlate of the źoom lensóf visual attention. Journal of Neuroscience 23(9): 3561-3565. 
Musseler, J., A.H. Heijden, S.H. Mahmud, H. Deubel, and S. Ertsey (1999). Relative mislocalization of briefly presented stimuli in the retinal periphery. Perception $\mathscr{6}$ Psychophysics 61(8): 1646-1661.

Nakamura, K. and C.L. Colby (2002). Updating of the visual representation in monkey striate and extrastriate cortex duing saccades. Proceedings of the National Academy of Science USA 99(6): 4026-4031.

Newsome, W. T., K. H. Britten, J. A. Movshon, and M. Shadlen (1989). Single neurons and the perception of visual motion. In Lam, D. M. and C. D. Gilbert, editors, Neural Mechanisms of Visual Perception, pp. 171-198. Houston, TX: Gulf Publishing Company, Houston, TX.

Newsome, W. T., R. H. Wurtz, and H. Komatsu (1988). Relation of cortical areas MT and MST to pursuit eye movements. II. differentiation of retinal from extraretinal inputs. Journal of Neurophysiology 60(2): 604-620.

Niebur, E. and C. Koch (1998). Computational architectures for attention. In R, Parasuraman, editor, The attentive brain, chapter 9, pp. 163-186. MIT Press, Cambridge, Massachussetts, 1st edition.

Noesselt, T., S.A. Hillyard, M.G. Woldorff, A. Schoenfeld, T. Hagner, L. Jancke, C. Tempelmann, H. Hinrichs, and H.J. Heinze (2004). Delayed striate cortical activation during spatial attention. Neuron 35(3): 575-587.

Nowlan, S.J. and T.J. Sejnowski (1995). A selection model for motion processing in area MT of primates. Journal of Neuroscience 15(2): 1195-1214.

O'Connor, D.H., M.M. Fukui, and M.A. Pinsk (2002). Attention modulates responses in the human lateral geniculate nucleus. Nature Neuroscience 11(6): 1203-1209.

O'Craven, K.M. and P.E. Downing (1999). fMRI evidence for objects as the units of attentional selection. Nature 401: 584-587.

Olshausen, B.A., C.H. Anderson, and D.C. Essen (1993). A neurobiological model of visual attention and invariant pattern recognition based on dynamic routing of information. Journal of Neuroscience 13: 4770-4719.

Olson, C.R. (2001). Object-based vision and attention in primates. Current Opinion in Neurobiology 11: 171-179.

O'Murray, S. and E. Wojciuluk (2003). Attention increases neural selectivity in the human lateral occipital cortex. Nature Neuroscience 7(1): 70-74. 
O'Regan, J.K. and R.A. Rensink (1999). Change-blindness as a result of 'mudsplashes'. Nature 398: 34.

Pack, C.C. and R.T. Born (2001). Temporal dynamics of a neural solution to the aperture problem in visual area MT of macaque brain. Nature 409: 1040-1042.

Pascual-Leone, A. and V. Walsh (2001). Fast backprojections from the motion to the primary visual area necessary for visual awareness. Science 292: 510-512.

Pena, J.L and M Konishi (2001). Auditory spatial receptive fields created by multiplication. Science 292: 249-252.

Perge, J.A., B. Borghuis, J.A. Duijnhouwer, M.J.M. Lankheet, and R.J.A. Wezel (2002). Direction tuning of macaque MT neurons: a reverse correlation study. Journal of Vision 2(7): 39a.

Pernberg, J., K.U. Jirmann, and U.T. Eysel (1998). Structure and dynamics of receptive fields in the visual cortex of the cat (area 18) and the influence of gabaergic inhibition. European Journal of Neuroscience 10(12): 3596-3606.

Perrone, J.A. and A. Thiele (2001). Speed skills: Measuring the visual speed analyzing properties of primate MT neurons. Nature Neuroscience 4(5): 526-532.

Pessoa, L., S. Kastner, and L.G. Ungerleider (2003). Neuroimaging studies of attention: from modulation of sensory processing to top-down control. Journal of Neursocience 23(10): 3990-3998.

Pettet, M. W. and C. D. Gilbert (1992). Dynamic changes in receptive fiel size in cat primate visual cortex. Proceedings of the National Academy of Science, USA 89(17): 8366-8370.

Pinsk, M.A., G.M. Doniger, and S. Kastner (2004). A push-pull mechanism of selective attention in human extrastriate cortex. Journal of Neurophysiology in press.

Platt, M.L. and P.W. Glimcher (1998). Response fields of intraparietal neurons quantified with multiple saccadic targets. Experimental Brain Research 121: 65-75.

Polat, U. (1999). Functional architecture of long-range perceptual interaction. Spatial Vision 12(2): 143-162.

Polat, U., K. Mizobem, and T. Kasamatsu (1998). Collinear stimuli regulate visual responses depending on cell's contrast threshold. Nature 391: 580-584.

Posner, M., C. Snyder, and B. Davidson (1980). Attention and the detection of signals. Journal of Experimental Psychology: General 109: 160-174. 
Pouget, A. and T.J. Sejnowski (1997). A new view of hemineglect based on the response properties of parietal neurons. Philosophical Transactions of the Royal Society London, BS 352: 149-1459.

Powell, K. D. and M. E. Goldberg (2000). Responses of neurons in the lateral intraprietal area to a distractor flashed during the delay period of a memoryguided saccade. Journal of Neurophysiology 84: 301-310.

Press, W.A., A.A. Brewer, R.F. Dougherty, A.R. Wade, and B.A. Wandell (2001). Visual areas and spatial summation in human visual cortex. Vision Research 41(10-11): 1321-1332.

Press, W.H., S.A. Teukolsky, W.T. Vetterling, and B.P. Flannery, editors (1988). Numerical Recipies in C. Cambridge University Press, Cambridge, USA, 2nd edition.

Priebe, N.J., M.M. Churchland, and S.G. Lisberger (2002). Constrains on the source of short-term motion adaptation in macaque area MT. I. The role of input and intrinsic mechanisms. Journal of Neurophysiology 88: 354-369.

Prinzmetal, W. and A. Wilson (1997). The effect of attention on phenomenal length. Perception 26(2): 193-205.

Raiguel, S., van M. Hulle, D.K. Xiao, V.L. Marcar, and G.A. Orban (1995). Shape and spatial distribution of receptive fields and antagonistic motion surrounds in area MT (V5) of the macaque. European Journal of Neuroscience 7: 2064-2082.

Raiguel, S., L. Lagae, B. Gulyos, and G.A. Orban (1989). Response latencies of visual cells in macaque areas V1, V2, and V5. Brain research 493: 155-159.

Raiguel, S.E., D.K. Xiao, V.L. Marcar, and G.A. Orban (1999). Response latency of macaque area MT/V5 neurons and its relation to stimulus parameters. Journal of Neurophysiology 82: 1944-1956.

Recanzone, G. H. and R. H. Wurtz (1999). Shift in smooth pursuit initiation and MT and MST neuronal activity under different stimulus conditions. Journal of Neurophysiology 82(4): 1710-1727.

Recanzone, G. H. and R. H. Wurtz (2000). Effects of attention on MT and MST neuronal activity during pursuit initiation. Journal of Neurophysiology 83(2): 777-790.

Recanzone, G.H. and R.H. Wurtz (1997). Responses of MT and MST neurons to one and two moving objects in the receptive field. Journal of Neurophysiology 78: 2904-2915. 
Rensink, R.A., J.K. O'Regan, and J.J. Clark (1997). To see or not to see: The need for attention to perceive changes in scenes. Psychological Science 8(5): 368-373.

Ress, D. and B.T. Backus (2000). Activity in primary visual cortex predicts performance in a visual detection task. Nature Neuroscience 3(9): 940-945.

Reynolds, J.H., L. Chelazzi, and R. Desimone (1999). Competitive mechanisms subserve attention in macaque areas V2 and V4. Journal of Neuroscience 19(5): 1736-1753.

Reynolds, J.H. and R. Desimone (1999). The role of neural mechanims of attention in solving the binding problem. Neuron 24: 19-29.

Reynolds, J.H. and R. Desimone (2003). Interacting roles of attention and visual salience in V4. Neuron 37(5): 853-63.

Reynolds, J.H., T. Pasternak, and R. Desimone (2000). Attention increases stimulus sensitivity of V4 neurons. Neuron 26: 703-714.

Richmond, B.J., R.H. Wurtz, and T. Sato (1983). Visual responses of inferior temporal neurons in awake rhesus monkey. Journal of Neurosphysiology 50: 1415-1432.

Rizzolatti, G., L. Riggio, I. Dascola, and C. Umilta (1987). Reorienting attention across the horizontal and vertical meridians: evidence in favor of a premotor theory of attention. Neuropsychologia 25(1A): 31-40.

Robinson, D.L., E.M. Bowman, and G.B. Stanton (1995). Covert orienting of attention in macaques. II. contributions of parietal cortex. Journal of Neurophysiology 74(2): 698-712.

Robinson, D.L., M.E. Goldberg, and G.B. Stanton (1978). Parietal association cortex in the primate: Sesnory mechanisms and behavioral modulations. Journal of Neurophysiology 41: 910-932.

Rodman, H.R. and T.D. Albright (1987). Coding of visual stimulus velocity in area MT of the macaque. Vision Research 27: 2035-2048.

Rodman, H.R., C.G. Gross, and T.D. Albright (1989). Afferent basis of visual response properties in area MT of the macaque. i. effects of striate cortex removal. Journal of Neuroscience 9(6): 2033-2050.

Rodman, H.R., C.G. Gross, and T.D. Albright (1990). Afferent basis of visual response properties in area MT of the macaque: II. effects of superior colliculus removal. Journal of Neuroscience 10: 1154-1164. 
Roelfsema, P.R. and V.A.F. Lamme (1998). Object-based attention in the primary visual cortex of the macaque monkey. Nature 395: 376-381.

Rolls, E.T., N.C. Aggelopoulos, and F. Zheng (2003). The receptive fields of inferior temporal cortex neurons in natural scenes. Journal of Neuroscience 23(1): 339-348.

Rolls, E.T. and G. Deco (2002). Computational Neuroscience of Vision. Oxford University Press, Oxford, UK, 1st edition.

Rosa, M.G. (1997). Visuotopic organisation of primate extrastriate cortex. In Rockland, K.S. and J.H. Kaas, editors, Extrastriate Cortex in Primates, Vol. 12, pp. 127-203. Plenum Press, New York.

Roy, J.P. and H. Komatsu (1992). Disparity sensitivity of neurons in monkey extrastriate area mst. Journal of Neuroscience 12(7): 2478-2492.

Saenz, M., G.T. Buracas, and G.M. Boynton (2002). Global effects of feature-based attention in human visual cortex. Nature Neuroscience 5: 631-632.

Salinas, E. (2004). Fast remapping of sensory stimuli onto motor actions on the basis of contextual modulation. Journal of Neuroscience 24(5): 1113-1118.

Salinas, E. and L. F Abbott (2001). Coordinate transformations in the visual system: How to generate gain fields and what to compute with them. In Nicolelis, M.A.L., editor, Progress in Brain Research, pp. 175-190. Amsterdam B.V.: Elsevier Science, Amsterdam.

Salinas, E. and L.F. Abbott (1996). A model of multiplicative neural responses in parietal cortex. Proceedings of the National Academy of Science, USA 93(12): 11956-11961.

Salinas, E. and L.F. Abbott (1997). Invariant visual responses from attentional gain fields. Journal of Neurophysiology 77(6): 3267-3272.

Salinas, E. and P. Thier (2000). Gain modulation: A major computational principle of the central nervous system. Neuron 27(1): 15-21.

Salzman, C.D. and W.T. Newsome (1994). Neural mechanisms for forming a perceptual decision. Science 264: 231-237.

Sasaki, Y., N. Hadjikhani, B. Fischl, A.K. Liu, S. Marrett, and A.M. Dale (2001). Local and global attention are mapped retinotopically in human occipital cortex. Proceedings of the National Academy of Science USA 98(4): 2077-2082. 
Schall, J.D. and D.P. Hanes (1993). Neural basis of saccade target selection in frontal eye field during visual search. Nature 366: 467-469.

Schmolensky, M.T., Y. Wang, D.P. Hanes, K.G. Thompson, S. Leutgeb, J.D. Schall, and A.G. Lventhal (1998). Signal timing across the macaque visual system. Journal of Neurophysiology 79: 3272-3278.

Schoenfeld, M.A., C. Tempelmann, A. Martinez, J.M. Hopf, C Sattler, H.J. Heinze, and S.A. Hillyard (2003). Dynamics of feature binding during object-selective attention. Proceedings of the National Academy of Science, USA 100(20): 11806-11811.

Scholl, B.J. (2001). Objects and attention: the state of the art. Cognition 80: 1-46.

Schweigart, G. and U.T. Eysel (2002). Activity-dependent receptive field changes in the surround of adult cat visual cortex lesions. European Journal of Neuroscience 15: 1585-1596.

Sclar, G. and R.D. Freeman (1982). Orientation selectivity in the cat's striate cortex is invariant with stimulus contrast. Experimental Brain Research 46: 457-461.

Sclar, G., J.H.R. Maunsell, and P. Lennie (1990). Coding of image contrast in central visual pathways of the macaque monkey. Vision Research 30(1): 1-10.

Seidemann, E. and W.T. Newsome (1999). Effect of spatial attention on the responses of area MT. Journal of Neurophysiology 81(4): 1783-1794.

Seidemann, E., E. Zohary, and W.T. Newsome (1998). Temporal gating of neural signals during performance of a visual discrimination task. Nature 394: 72-75.

Sengpiel, F. and A. Sen (1997). Characteristics of surround inhibition in cat area 17. Experimental Brain Research 116: 216-228.

Sengpiel, F. and P. Stawinski (1999). Influence of experience on orientation maps in cat visual cortex. Nature Neuroscience 2(8): 727-731.

Shadlen, M.N. and W.T. Newsome (2001). Neural basis of a perceptual decision in the parietal cortex (area LIP) of the rhesus monkey. Journal of Neuroscience 86: 1916-1936.

Ship, S. and S. Zeki (1989a). The organisation of connections between areas V5 and V1 in macaque monkey visual cortex. European Journal of Neuroscience 1: 309-332. 
Ship, S. and S. Zeki (1989b). The organisation of connections between areas V5 and V2 in macaque monkey visual cortex. European Journal of Neuroscience 1: 333-354.

Shulman, G.L., J. Wildon, and J.B. Sheehy (1985). Spatial determinants of the distribution of attention. Perception \& Psychophysics 37: 59-65.

Sigala, N. and N.K. Logothetis (2002). Visual categorization shapes feature selectivity in the primate temporal cortex. Nature 415: 318-320.

Simons, D.J., G.E. Carvell, A.E. Hershey, and D.P. Bryant (1992). Responses of barrel cortex neurons in awake rats and effects of urethane anesthesia. Experimental Brain Research 91(2): 259-272.

Slotnick, A.D., J.B. Hopfinger, S.A. Klein, and E.E. Sutter (2002). Darkness beyond the light: attentional inhibition surrounding the classic spotlight. Neuroreport 13: $773-778$.

Slotnick, A.D., J. Schwarzbach, and S. Yantis (2003). Attentional inhibition of visual processing in human striate and extrastriate cortex. Neuroimage 19: 1602-1611.

Smith, A.T. and K.D. Singh (2000). Attentional suppression of activity in the human visual cortex. Neuroreport 11(2): 271-277.

Snodderly, D.M. and I. Kagan (2001). Selective activation of visual cortex neurons by fixational eye movements. Visual Neuroscience 18: 259-277.

Snowden, R.J., S. Treue, and R.A. Andersen (1992). The response of areas V1 and MT of the alert rhesus monkey to moving random dot patterns. Experimental Brain Research 88: 389-400.

Snowden, R.J., S. Treue, R.E. Erickson, and R.A. Andersen (1991). The response of area MT and V1 neurons to transparent motion. Journal of Neuroscience 11(9): 2768-2785.

Sober, S.J., J.M. Stark, and D.S. Yamasaki (1997). Receptive field changes after strokelike cortical ablation: A role for activation dynamics. Journal of Neurophysiology 78: 3438-3443.

Somers, D.C., A.M. Dale, and A.E. Seifert (1999). Functional mri reveals spatially specific attentional modulation in human primary visual cortex. Proceeding of the National Academy of Science USA 96: 1663-1668.

Spitzer, H., R. Desimone, and J. Moran (1988). Increased attention enhances both behavioral and neuronal performance. Science 240: 338-340. 
Spitzer, H. and B. J. Richmond (1991). Task difficulty: ignoring, attending to, and discriminating a visual stimulus yield progressively more activity in inferior temporal neurons. Experimental Brain Research 83(2): 340-348.

Steinmetz, M. A., C. E. Connor, and C. Constantinidis (1994). Covert attention suppresses neuronal responses in area $7 \mathrm{a}$ of the posterior parietal cortex. Journal of Neurophysiology 72(2): 1020-1023.

Steinmetz, M. A. and C. Constantinidis (1995). Neurophysiological evidence for a role of posterior parietal cortex in redirecting visual attention. $C C$ 5: 448-456.

Suder, K. and F. Wörgötter (2000). The control of low-level information flow in the visual system. Reviews in the Neurosciences 11: 127-146.

Suzuki, S. and P. Cavanagh (1997). Focussed attention distorts visual space: An attentional repulsion effect. Journal of Experimental Psychology: Human Perception \& Performance 23: 443-463.

Tanaka, K., K. Hikosaka, H. Saito, M. Yukie, and J. Fukada (1986). Analysis of local and wide-field movements in the primate temporal visual areas of the macaque monkey. Journal of Neuroscience 6: 134-144.

Tanaka, K., Y. Sugita, M. Moriya, and H.A. Saito (1993). Analysis of object motion in the ventral part of the medial superior temporal area of the macaque visual cortex. Journal of Neurophysiology 69(1): 128-142.

Thiele, A., P. Henning, M. Kubischik, and K.P. Hoffmann (2002). Neural mechanisms of saccadic suppression. Science 295: 2460-2462.

Tolhurst, D.J. (1973). Separate channels for the analysis of the shape and the movement of a moving visual stimulus. Journal of Physiology 231: 385-402.

Tolias, A.S., T. Moore, S.M. Smirnakis, E.J. Tehovnik, A.G. Siapas, and P.H. Schiller (2001). Eye movements modulate visual receptive fields of V4 neurons. Neuron 29(3): 757-767.

Tootell, R.B., M.S. Silverman, R.L. De Valois, and G.H. Jacobs (1983). Functional organisation of the second functional area in primates. Science 220: 737-739.

Tootell, R.B.H., N. Hadjikhani, E.K. Hall, S. Marrett, W. Vanduffel, and J.T. Vaughan (1998). The retinotopy of visual spatial attention. Neuron 21: 1409-1422.

Tootell, R.B.H., D. Tsao, and W. Vanduffel (2003). Neuroimaging weights in: Humans meet macaques in "primate" visual cortex. Journal of Neuroscience 23: 3981-3989. 
Toth, L.J. and J.A. Assad (2002). Dynamic coding of behaviourally relevant stimuli in parietal cortex. Nature 415: 165-168.

Treisman, A. (1998). Feature binding, attention and object perception. Philosophical Transactions of the Royal Society London, BS 353: 1295-1306.

Treisman, A. and G. Gelade (1980). A feature-integration theory of attention. Cognitive Psychology 12: 97-136.

Treue, S. (2001). Neural correlates of attention in primate visual cortex. Trends in Neurosciences 24(5): 295-300.

Treue, S. (2003). Visual attention: The where, what, how, and why of saliency. Current Opinion in Neurobiology 13: 428-432.

Treue, S., K. Hol, and H.-J. Rauber (2000). Seeing multiple directions of motion physiology and psychophysics. Nature Neuroscience 3(3): 270-276.

Treue, S. and J.C. Martinez-Trujillo (1999). Feature-based attention influences motion processing gain in macaque visual cortex. Nature 399: 575-579.

Treue, S. and J. H. R. Maunsell (1999). Effects of attention on the processing of motion in macaque visual cortical areas MT and MST. Journal of Neuroscience 19(17): 7603-7616.

Treue, S. and J.H. Maunsell (1996). Attentional modulation of visual motion processing in cortical areas MT and MST. Nature 382: 539-541.

Tsal, Y. and T. Bareket (1999). Effects of attention on localization of stimuli in the visual field. Psychonomic Bulletin \& Review 6(2): 292-296.

Tsal, Y. and N. Lavie (1988). Attending to color and shape: The special role of location in selective visual processing. Perception \&f Psychophysics 19: 15-21.

Tsal, Y. and L. Shalev (1996). Inattention magnifies perceived length: The attentional receptive field hypothesis. Journal of Experimental Psychology: Human Perception \& Performance 22(1): 233-243.

Tse, P.U., D.L. Sheinberg, and N.K. Logothetis (2003). Attentional enhancement opposite a peripheral flash revealed using change blindness. Psychological Science 14(2): 91-99.

Tsotsos, J.K., S.M. Culhane, W.Y.K. Wai, Y. Lai, N. Davis, and F Nuflo (1995). Modeling visual attention via selective tuning. Artifical Inteligence 78: 507-545. 
Umeno, M.M. and M.E. Goldberg (1997). Spatial processing in the monkey frontal eye field: 1. Predictive visual responses. Journal of Neurophysiology 78: 1373-1383.

Ungerleider, L.G. and R. Desimone (1986). Projections to the superior temporal sulcus from the central and peripheral field representations of V1 and V2. Journal of Comparative Neurology 248(2): 147-163.

Ungerleider, L.G., R. Desimone, T.W. Galkin, and M. Mishkin (1984). Subcortical projections of area MT in the macaque. Journal of Comparative Neurology 223: 368-386.

Vajda, I. (2003). Single-unit studies of visual motion processing in cat extrastriate cortex. Ph.D. diss., Univerity of Utrecht, Department of Biology, Utrecht, Netherland.

van Essen, D. A. and J. H. R. Maunsell (1983). Hierarchical organization and functional streams in the visual cortex. Journal of Neuroscience pp. 370-375.

van Wezel, R.J.A. and K. H. Britten (2002). Motion adaptation in area MT. Journal of Neurophysiology 88: 3469-3476.

van Wezel, R.J.A., M.J.M. Lankheet, F.A.J. Verstraten, A.F.M. Maree, and W.A. Grind (1996). Responses of complex cells in area 17 of the cat to bi-vectorial transparent motion. Visual Research 36(18): 2805-2813.

Vidyasagar, T.R. (1998). Gating of neuronal responses in macaque primary visual cortex by an attentional spotlight. Neuroreport 9(9): 1947-1952.

Volchan, E. and C.D. Gilbert (1995). Interocular transfer of receptive field expansion in cat visual cortex. Vision Research 35(1): 1-6.

Walker, M.F., E.J. Fitzgibbon, and M.E. Goldberg (1995). Neurons in the monkey superior colliculus predict the visual result of impending saccadic eye movements. Journal of Neurophysiology 73: 1988-2003.

Wallis, G. and H. Buelthoff (1999). Learning to recognize objects. Trends in Cognitive Science 3(1): 22-31.

Wang, Y., I. Fujita, H. Tamura, and Y. Murayama (2002). Contribution of GABAergic inhibition to receptive field structures of monkey inferior temporal neurons. Cerebral Cortex 12(1): 62-74.

Watanabe, T., A.H. Harner, S. Miyauchi, and Y. Sasaki (1998). Task-dependent influences of attention on the activation of human primary visual cortex. Proceedings of the national Academy of Science, USA 95: 11589-11492. 
Weinberger, N.M. (1995). Dynamic regulation of receptive fields and maps in the adult sensory cortex. Annual Reviews in Neuroscience 18: 129-158.

Williams, Z.M., J.C. Elfar, E.N. Eskandar, L.J. Toth, and J.A. Assad (2003). Parietal activity and the perceived direction of ambiguous apparent motion. Nature Neuroscience 6(6): 616-623.

Wojciulik, E. and N. Kanwisher (1999). The generality of parietal involvement in visual attention. Neuron 23: 747-764.

Wörgötter, F. and U. T. Eysel (2000). Context, state and the receptive fields of striatal cortex cells. Trends in Neuroscience 23(10): 497-503.

Wörgötter, F., K. Suder, Y. Zhao, N. Kerscher, and U.T. Eysel (1998). Statedependent receptive-field restructuring in the visual cortex. Nature 396: 165-166.

Wurtz, R. H. and M. E. Goldberg (1980). Behavioral modulation of visual responses in the monkey: Stimulus selection for attention and movement. Progress in Psychobiology \& Physiological Psychology 9: 43-83.

Wurtz, R.H., D.S. Yamasaki, C.J. Duffy, and J.P. Roy (1990). Functional specialization for visual motion processing in primate cerebral cortex. Cold Spring Harbor Smposia on Quantitative Biology LV: 717-727.

Xiang, J.Z., V.L. Marcar, and S.E. Raiguel (1997). Selectivity of macaque MT/V5 neurons for surface orientation in depth. European Journal of Neuroscience 9: 956-964.

Xiao, D.K., V.L. Marcar, and S.E. Raiguel (1997). The spatial distribution of the antagonistic surrounds of area MT/V5 neurons. Cerebral Cortex 7: 662-677.

Xiao, D.K., V.L. Marcar, and S.E. Raiguel (1998). Influence of stimulus speed upon antagonistic surrounds of area MT/V5 neurons. Neuroreport 9: 1321-1326.

Xiao, D.K., S. Raiguel, V.L. Marcar, and J. Koenderink (1995). Spatial heterogenitiy of inhibitory surrounds in the middle temporal visual area. Proceedings of the National Academy of Science USA 92: 11303-11306.

Xing, J. and G.L. Gerstein (1994). Simulation of dynamic receptive fields in primary visual cortex. Vision Research 34(14): 1901-1911.

Yantis, S. (1998). Control of visual attention. In Pashler, H., editor, Attention, chapter 6, pp. 223-256. 
Yantis, S. and J. Jonides (1990). Abrupt visual onsets and selective attention: voluntary versus automatic allocation. Journal of Experimental Psychology: Human Perception \& Performance 16(1): 121-134.

Yantis, S. and J.T. Serences (2003). Cortical mechanisms of space-based and objectbased attentional control. Current Opinion in Neurobiology 13(2): 187-93.

Yarbus, A.L. (1967). Eye movements and vision. Plenum, New York, 1st edition.

Yeshurun, Y. and M. Carrasco (1998). Attention improves or impairs visual performance by enhancing spatial resolution. Nature 396: 72-75.

Yeshurun, Y. and M. Carrasco (1999). Spatial attention improves performance in spatial resolution tasks. Vision Research 39(2): 293-306.

Yeshurun, Y. and M. Carrasco (2000). The locus of attentional effects in texture segmentation. Nature Neuroscience 3(6): 622-627.

Yin, T.C.T. and V.B. Mountcastle (1978). Mechanisms of neural integration in the parietal lobe for visual attention. Federation Proceedings 37: 2251-2257.

Zar, J.H. (1999). Biostatistical Analysis. Prentice Hall, New Jersey, USA, 4th edition. 


\title{
Curriculum Vitae
}

\author{
name \\ Thilo Womelsdorf \\ date of birth \\ 29. January 1974 in Bad Oeynhausen, Germany \\ nationality \\ german \\ marital status \\ single
}

16.02 .2001

Begin PhD thesis, Cognitive Neuroscience Laboratory, Deutsches Primatenzentrum, Göttingen, Germany

15.02 .2001

Diploma in Psychology, Ruhr Universität Bochum, Germany

09/96- 07/97

Foreign studies in Psychology, University of Hull, United Kingdom

15.07 .1996

Pre-diploma in Psychology, Ruhr Universität Bochum, Germany

15.10 .1994

Beginn: Psychology Studies, Ruhr Universität Bochum, Germany

10/93 - 12/94 Civilian Duty, Third World Information Center, Herne Germany,

28.05.1993
Allgemeine Hochschulreife (A Levels), städtisches
Gymnasium Löhne, Westfalen, Germany 